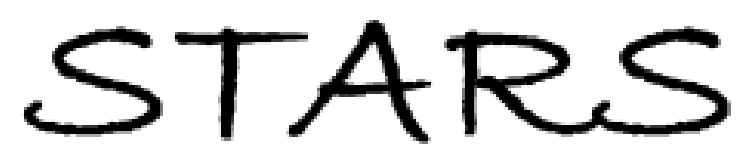

University of Central Florida

STARS

\title{
A Time-varying Radiometric Bias Correction For The Trmm Microwave Imager
}

Kaushik Gopalan

University of Central Florida

Part of the Electrical and Electronics Commons

Find similar works at: https://stars.library.ucf.edu/etd

University of Central Florida Libraries http://library.ucf.edu

This Doctoral Dissertation (Open Access) is brought to you for free and open access by STARS. It has been accepted for inclusion in Electronic Theses and Dissertations, 2004-2019 by an authorized administrator of STARS. For more information, please contact STARS@ucf.edu.

\section{STARS Citation}

Gopalan, Kaushik, "A Time-varying Radiometric Bias Correction For The Trmm Microwave Imager" (2008). Electronic Theses and Dissertations, 2004-2019. 3596.

https://stars.library.ucf.edu/etd/3596

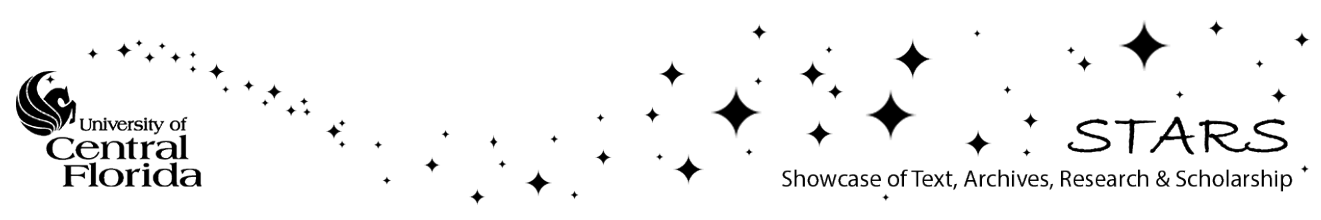




\section{A TIME-VARYING RADIOMETRIC BIAS CORRECTION FOR THE TRMM MICROWAVE IMAGER}

by

KAUSHIK GOPALAN

B.E Rashtreeya Vidyalaya College of Engineering, 2003

M.S.E.E. University of Central Florida, 2005

A dissertation submitted in partial fulfillment of the requirements

for the degree of Doctor of Philosophy

in the School of Electrical Engineering and Computer Science

in the College of Engineering and Computer Science

at the University of Central Florida

Orlando, Florida

Fall Term

2008

Major Professors: Linwood Jones

Takis Kasparis 
(c) 2008 by KAUSHIK GOPALAN 


\section{Abstract}

This dissertation provides a robust radiometric calibration for the TRMM Microwave Imager to correct systematic brightness temperature errors, which vary dynamically with orbit position (time) and day of the year. The presence of a time-varying bias in TMI is confirmed by inter-calibration with WindSat and SSMI. This time varying bias is manifested as a time of day dependent variation of the relative biases between TMI and both WindSat and SSMI. In this dissertation, we provide convincing evidence that this time-varying $\mathrm{Tb}$ bias in TMI is caused by variations in the physical temperature of the emissive TMI reflector antenna. This dissertation provides an empirical correction that largely corrects this timevarying bias. The TMI bias is estimated by comparing the $10.7 \mathrm{GHz} \mathrm{V}$-polarization channel observations with RTM Tb predictions, and the $\mathrm{Tb}$ correction is applied as a function of orbit time for every day of the one year period. Furthermore, this dissertation provides a qualitative physical basis for the estimated $\mathrm{Tb}$ bias patterns and provides conclusive evidence that the empirical correction applied to TMI Tb measurements (both ocean and land) largely corrects the time-varying TMI calibration. This is accomplished by demonstrating that the local time-of-day dependence (in the uncorrected TMI Tb values) is removed in the corrected TMI Tb's. 
Dedicated to the fond hope that the expression "Main Street" will forever be banned from political discourse in the United States. 


\section{ACKNOWLEDGMENTS}

I would first like to acknowledge the immense support and guidance I have received from my advisors, Dr. Linwood Jones and Dr. Takis Kasparis. I am especially grateful to Dr. Jones for the countless hours he has spent on improving my writing. I would also like to thank the members of my committee: Dr. Thomas Wilheit, Mr. James Johnson, Dr. John Lane and Dr. Michael Georgiopoulos for their contributions to my dissertation. I would like to thank the members of the CFRSL: Sayak Biswas, Spencer Farrar, Rafik Hanna, Pete Laupattarakasem, Salem El-Nimri, Ruba Amarin, Suleiman Alweiss and others for their help and support; and for being such great fun to be around. I'd like to make special mention of Liang Hong, who helped me greatly towards the beginning of my Ph.D.; and Sayak Biswas, who helped me out towards the end.

I have relied on many friends to get through graduate school; and they have my unending gratitude. I will not name them here, for I suspect that they know who they are. Finally, I'd like to thank my parents and my brother, who've never allowed me to feel distant, even when I've been thousands of miles away.

I wish to acknowledge that the financial support to pursue my Ph.D. program was sponsored by the NASA Science Mission Directorate, Earth Science Division under a grant 
for the Precipitation Measurements Mission science team support from the NASA Goddard Space Flight Center's Tropical Rainfall measuring Mission. 


\section{TABLE OF CONTENTS}

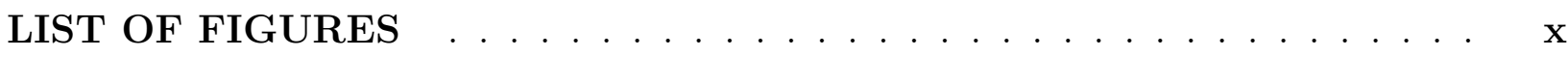



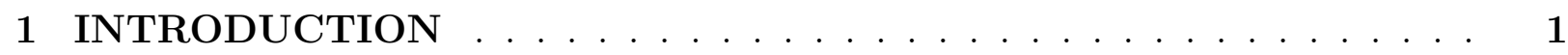

1.1 TRMM Satellite Description . . . . . . . . . . . . . . 3

1.2 TMI Brightness Temperature Anomaly . . . . . . . . . . . . . . . . 4

1.3 TMI Inter-satellite Calibration . . . . . . . . . . . . . . . . . 6

1.4 Dissertation Purpose and Organization . . . . . . . . . . . . . 8

2 CROSS-CALIBRATION OF TMI, WINDSAT AND SSMI . . . . . . . 11

2.1 Frequency and Incidence Angle Normalization Using Taylor Series . . . . . . 12

2.2 ICWG Common Dataset . . . . . . . . . . . . . . . . . 15

2.3 Generation of Near-Simultaneous Collocations . . . . . . . . . . . . . . . 16

2.4 Radiative Transfer Modeling . . . . . . . . . . . . . . . . . 29

2.5 Radiometric Inter-comparison of TMI with Windsat and SSMI . . . . . . . . 36

3 TMI ORBITAL BIAS MODELING . . . . . . . . . . . . . . 66 
3.1 TMI Tb Modeling . . . . . . . . . . . . . . . . . . . . 70

3.1 .1 TMI Channel Selection . . . . . . . . . . . . . . . . . 71

3.1.2 Typical Environmental Parameters in 1 deg Boxes . . . . . . . . . . 78

3.1 .3 RTM Validation . . . . . . . . . . . . . . . . . . 81

3.1.4 TMI Tb Bias Determination . . . . . . . . . . . . . . . . 81

3.2 TMI Bias Averaging by Orbit Segments . . . . . . . . . . . . . . 87

3.3 Inter-comparison of Corrected TMI Tb's with WindSat and SSMI . . . . . . 103

4 SUMMARY AND CONCLUSIONS . . . . . . . . . . . . . 112

$4.1 \mathrm{TMI} \mathrm{Tb}$ Bias Errors . . . . . . . . . . . . . . . . . . 112

4.2 TMI Radiometric Correction . . . . . . . . . . . . . . . . . . . . 118

4.3 Error Sources . . . . . . . . . . . . . . . . . . . . . . . 121

4.4 Future Work . . . . . . . . . . . . . . . . . . . . . . . 123

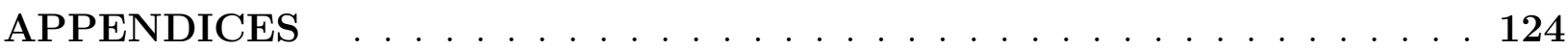

\section{A SATELLITE RADIOMETERS IN THE ICWG INTER-CALIBRATION}

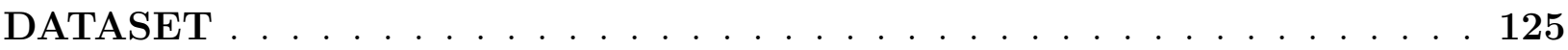

A.1 Special Sensor Microwave Imager (SSMI) . . . . . . . . . . . . . . . 125

A.2 TRMM Microwave Imager $(\mathrm{TMI}) \ldots \ldots \ldots \ldots$. . . . . . . . . 128

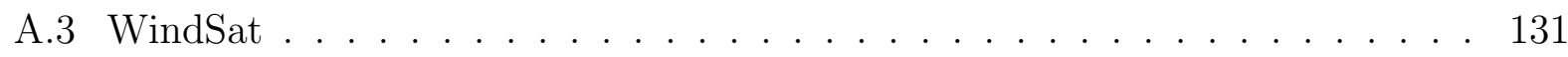


B NORMALIZATION OF WINDSAT TB'S TO TMI FREQUENCY AND INCIDENCE ANGLES . . . . . . . . . . . . . . . . . . 134

C TMI OBSERVATIONS SEPARATED BY TIME OF DAY . . . . . . 141

D EFFECT OF DERIVED TMI TB CORRECTION ON H-POL TMIWINDSAT RADIOMETRIC BIASES . . . . . . . . . . . . . . . . . 148

LIST OF REFERENCES _ . . . . . . . . . . . . . . . . . . . 152 


\section{LIST OF FIGURES}

1.1 The TRMM Observatory. . . . . . . . . . . . . . . . . 4

$2.1 \mathrm{~Tb}$ spectrum example from Hong $[10] . \ldots \ldots \ldots$. . . . . . . . . . . 13

2.2 Generation of Match-up Dataset. . . . . . . . . . . . . . 17

2.3 Geo-location of TMI-WindSat collocations. . . . . . . . . . . . . . . . 20

2.3 Geo-location of TMI-WindSat collocations. . . . . . . . . . . . . . . . . 21

2.4 Geo-location of TMI - SSMI collocations. . . . . . . . . . . . . . . 22

2.5 Effect of the filtering process on TMI-WindSat collocations. . . . . . . . . . 27

2.5 Effect of the filtering process on TMI-WindSat collocations. . . . . . . . . . 28

2.6 Variation of $10.7 \mathrm{GHz}$ surface emission with wind speed. . . . . . . . . . 30

2.7 Variation of $10.7 \mathrm{GHz}$ surface emission with $\mathrm{SST} \ldots \ldots \ldots$. . . . . . . 31

2.8 Frequency spectrum of WV absorption. . . . . . . . . . . . . . . . 33

2.9 Frequency spectrum of CLW absorption. . . . . . . . . . . . . . 33

2.10 Frequency spectrum of $\mathrm{N} 2$ absorption. . . . . . . . . . . . . . . 34

2.11 Frequency spectrum of $\mathrm{O} 2$ absorption at sea level pressure. . . . . . . . . . . 34

2.12 Normalized WindSat 10V Tb observations to corresponding TMI channel frequency and incidence angle. . . . . . . . . . . . . . . . . . 38 
2.12 Normalized WindSat 10V Tb observations to corresponding TMI channel frequency and incidence angle. . . . . . . . . . . . . . . . . . 39

2.13 Normalized WindSat 37V Tb observations to corresponding TMI channel frequency and incidence angle. . . . . . . . . . . . . . . . 41

2.13 Normalized WindSat 37V Tb observations to corresponding TMI channel frequency and incidence angle. . . . . . . . . . . . . . . . . . . . . 42

2.14 TMI and WindSat 10V unexplained Tb bias distribution. . . . . . . . . . 43

2.15 TMI-WindSat bias SST dependence for V-pol channels. . . . . . . . . . . . . 44

2.15 TMI-WindSat bias SST dependence for V-pol channels. . . . . . . . . . . . . 45

2.16 TMI-WindSat bias SST dependence for H-pol channels. . . . . . . . . . . . . 46

2.16 TMI-WindSat bias SST dependence for H-pol channels. . . . . . . . . . . . . 47

2.17 Theoretical ocean surface brightness temperature for an incidence angle of 53 deg and an ocean wind speed of $6 \mathrm{~m} / \mathrm{s}$ and a salinity of 32 ppt. . . . . . 48

2.18 TMI-WindSat predicted and measured Tb differences. . . . . . . . . . . . 50

2.19 TMI/WindSat Incidence angle difference variation with latitude. . . . . . . . 52

2.20 TMI incidence angle variation with scan azimuth position. . . . . . . . . 53

2.21 Latitude dependence of TMI-WindSat 10V measured Tb differences. . . . . . 54

2.22 TMI-WindSat bias dependence on columnar WV . . . . . . . . . . . . 55

2.23 WV dependence of TMI-WindSat 10V measured Tb differences. . . . . . . . 56

2.24 GDAS SST diurnal variation. . . . . . . . . . . . . . . . . . 57 
2.25 Evening - Morning measured Tb differences separately for TMI and WindSat. 60

2.26 Evening and Morning TMI - WindSat radiometric bias histograms. . . . . . 61

2.27 Radiometric calibration differences for Evening and morning collocations between TMI and WindSat/SSMI. The monthly averages given as red "dot' symbols are evening And the blue "X" symbols are morning. . . . . . . . . .

2.27 Radiometric calibration differences for Evening and morning collocations between TMI and WindSat/SSMI. The monthly averages given as red 'dot' symbols are evening and the blue 'X' symbols are morning. . . . . . . . . . . 64

3.1 Local time of day variation over a typical TMI orbit. . . . . . . . . . . . . 69

3.2 Incidence angle dependence of $10 \mathrm{~V}$ surface emission. . . . . . . . . . . . . 72

3.3 Incidence angle dependence of $10 \mathrm{H}$ surface emission. . . . . . . . . . . . . 72

3.4 Reflected downwelling atmospheric Tb's for an ensemble of 63 profiles for the $10 \mathrm{~V}$ and $10 \mathrm{H}$ channels. . . . . . . . . . . . . . . . . . 74

3.5 Modeled ocean apparent $\mathrm{Tb}$ for an ensemble of 63 atmospheric profiles. . . . 76

3.6 Modeled ocean apparent Tb for an ensemble of 9 cloud models. . . . . . . . 76

3.7 Modeled ocean apparent Tb for 7 handbook atmospheres. . . . . . . . . . . . 77

3.8 Typical histograms of WS, WV and CLW for rain-free ocean scenes. . . . . . 79

3.8 Typical histograms of WS, WV and CLW for rain-free ocean scenes in the match-up dataset. . . . . . . . . . . . . . . . . . 80 
3.10 Results of filtering based on upper limit Tb thresholds, std. dev. thresholds and CLW. . . . . . . . . . . . . . . . . . . 85

3.10 Results of filtering based on upper Tb thresholds, std. dev. thresholds and CLW.

3.11 TMI ground swath for one orbit with markers at five-minute intervals. . . . . 88

3.12 Standard deviation of the estimate of the $\mathrm{Tb}$ bias for a one-month average

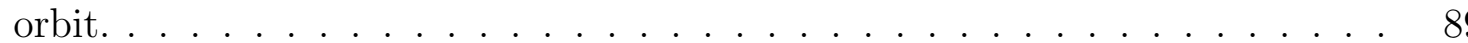

3.13 Orbital pattern for TMI 10V bias averaged over one day for two different seasons.

3.14 Estimated TMI reflector temperature variation over an orbit. . . . . . . . . 92

3.15 Daily TMI bias characteristics (July 1, 2005 - August 17, 2005) . . . . . . 94

3.16 STK analysis of TRMM spacecraft shadowing for July 18, 2005. . . . . . . . 95

3.16 STK analysis of TRMM spacecraft shadowing for July 18, 2005. . . . . . . . 96

3.16 STK analysis of TRMM spacecraft shadowing for July 18, 2005. . . . . . . . 97

3.17 STK analysis of TRMM spacecraft shadowing for August 2, 2005. . . . . . . 99

3.17 STK analysis of TRMM spacecraft shadowing for August 2, 2005. . . . . . . 100

3.17 STK analysis of TRMM spacecraft shadowing for August 2, 2005. . . . . . . 101

3.18 Mean daily bias variation for 2004 and 2005. . . . . . . . . . . . . . . . 102

3.19 Daily peak-to-peak bias variation for 2004 and 2005 . . . . . . . . . . . 102 
3.20 Relative radiometric biases with WindSat for corrected TMI Tb's. . . . . . . 106

3.20 Relative radiometric biases with WindSat for corrected TMI Tb's. . . . . . . 107

3.21 Relative radiometric biases with SSMI F13 for corrected TMI Tb's. . . . . . 108

3.21 Relative radiometric biases with SSMI F13 for corrected TMI Tb's. . . . . . 109

3.22 Relative radiometric biases with SSMI F14 for corrected TMI Tb's. . . . . . 110

3.22 Relative radiometric biases with SSMI F14 for corrected TMI Tb's. . . . . . 111

4.1 Daily estimated TMI Tb bias patterns. . . . . . . . . . . . 115

4.2 Daily estimated TMI Tb bias patterns. . . . . . . . . . . . 116

4.3 Daily estimated TMI Tb bias patterns. . . . . . . . . . . . 117

4.4 TRMM 1B11 version 7 bias correction. . . . . . . . . . . . . . . . . 120

A.1 SSMI scan geometry . . . . . . . . . . . . . . . 126

A.2 SSMI reflector positioning . . . . . . . . . . . . 127

A.3 TMI scan geometry . . . . . . . . . . . . . . . . . . . . . . . . . 129

A.4 TMI footprint characteristics . . . . . . . . . . . . . . . 130

A.5 Azimuth distribution of TMI scan sectors . . . . . . . . . . . . . . . 131

A.6 WindSat sensor assembly . . . . . . . . . . . . . . . . . . 133

B.1 Normalized WindSat 10H Tb observations to corresponding TMI channel frequency and incidence angle. . . . . . . . . . . . . . . . 135 
B.2 Normalized WindSat $18 \mathrm{~V}$ Tb observations to corresponding TMI channel frequency and incidence angle. . . . . . . . . . . . . . . . 136

B.3 Normalized WindSat 19H Tb observations to corresponding TMI channel frequency and incidence angle. . . . . . . . . . . . . . . . . . 137

B.4 Normalized WindSat 23V Tb observations to corresponding TMI channel frequency and incidence angle. . . . . . . . . . . . . . . . . . . 138

B.5 Normalized WindSat 37V Tb observations to corresponding TMI channel frequency and incidence angle.

B.6 Normalized WindSat 37H Tb observations to corresponding TMI channel frequency and incidence angle. . . . . . . . . . . . . . . . . . . . . 140

C.1 TMI 10V Tb observed Evening - Morning difference. . . . . . . . . . . . . . 141

C.2 TMI 10H Tb observed Evening - Morning difference. . . . . . . . . . . . . . 142

C.3 TMI 19V Tb observed Evening - Morning difference. . . . . . . . . . . . . . 143

C.4 TMI 19H Tb observed Evening - Morning difference. . . . . . . . . . . . . . 144

C.5 TMI 21V Tb observed Evening - Morning difference. . . . . . . . . . . . . 145

C.6 TMI 37V Tb observed Evening - Morning difference. . . . . . . . . . . . . 146

C.7 TMI 37H Tb observed Evening - Morning difference. . . . . . . . . . . . . . 147

D.1 Relative $10 \mathrm{H}$ radiometric biases with WindSat for uncorrected and corrected

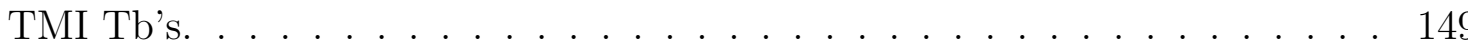


D.2 Relative $19 \mathrm{H}$ radiometric biases with WindSat for uncorrected and corrected TMI Tb's. . . . . . . . . . . . . . . . . . 150

D.3 Relative $37 \mathrm{H}$ radiometric biases with WindSat for uncorrected and corrected TMI Tb's. . . . . . . . . . . . . . . . . . . . . 151 


\section{LIST OF TABLES}

1.1 Slopes and Intercepts from SSMI inter-comparison from Wentz [7]. . . . . . . 6

2.1 Composition of TMI - WindSat collocation files. . . . . . . . . . . 18

2.2 Composition of TMI - SSMI collocation files. . . . . . . . . . . . . 19

2.3 Upper limits for TMI (SSMI) channels for oceanic scenes. . . . . . . . . . . 26

2.4 Upper limits for WindSat channels for oceanic scenes. . . . . . . . . . . . . 26

2.5 Relative bias between TMI and WindSat/SSMI . . . . . . . . . . . 64

3.1 Cloud models . . . . . . . . . . . . . . . . . . . . . . 74

3.2 Relative bias for corrected TMI Tb's . . . . . . . . . . . . . 105

A.1 DMSP satellites with SSM/I radiometers . . . . . . . . . . . 125

A.2 SSMI Characteristics . . . . . . . . . . . . . . 126

A.3 TMI Characteristics . . . . . . . . . . . . . . . . . . 129

A.4 WindSat Characteristics . . . . . . . . . . . . . . . 132 


\section{INTRODUCTION}

The Tropical Rainfall Measurement Mission (TRMM) is a joint space program between the National Aeronautics and Space Administration (NASA) and the Japan Aerospace Exploration Agency (JAXA). As a major satellite program in NASA's "Mission to Planet Earth", TRMM has the scientific objective to better understand the Earth's hydrological cycle of which tropical rainfall is a major component. In fact, TRMM is the first satellite mission dedicated to observing the statistics of rainfall (especially over oceans) and to the understanding how this affects the Earth's global climate [1-3].

Originally, planned as a 3-year mission, the success of TRMM has exceeded expectations; and as a result in 2001, NASA extended the TRMM mission by raising the satellite altitude to prolong its life. Further, in 2004, TRMM was threatened with a termination of mission by NASA; but this was avoided by a strong endorsement by the scientific community $[4,5]$ including the National Academy of Sciences which wrote [6] “. . . In December 2004, the committee released Assessment of the Benefits of Extending the Tropical Rainfall Measuring Mission: A Perspective from the Research and Operations Communities, Interim Report (NRC, 2004). Because of TRMM's unique and substantial contributions to the research and operational communities, the committee recommended its continued operation. The National Aeronautics and Space Administration agreed with this recommendation, and TRMM was extended to at least fiscal year 2009. The possibility remains for TRMM to operate until its fuel runs out in approximately 2012."

This report went on to describe the benefit of TRMM to the future Global Precipitation Mission (GPM). The TRMM mission was the first NASA satellite mission to demon- 
strate the feasibility of measuring tropical precipitation from space. It has also demonstrated the value of the precipitation radar in observing the three-dimensional structure of precipitating weather systems. Further, TRMM is similar to the GPM core observatory in that it is a non-sun-synchronous tropical satellite that carries both a microwave radiometer and a precipitation radar. This allows the TRMM satellite to be used as a proxy for the GPM observatory in GPM mission efforts to improve calibration and retrieval techniques before the launch of the GPM core satellite. Improvements in these techniques will be extremely useful in applications that include numerical weather prediction, tropical cyclone and severe storm monitoring, flash flood forecasting, global precipitation analyses and precipitation climatology.

TRMM can be used in conjunction with GPM to produce multi-decadal climate data records for climate change study. Additionally, the constellation of TRMM and currently operational polar-orbiting satellite missions such as Coriolis and DMSP can provide nearreal-time global coverage in the pre-GPM era. However, the sensors in Coriolis and DMSP are built with different technology from TMI, which means that it is possible that they measure slightly different $\mathrm{Tb}$ values even while viewing similar precipitation scenes. Further, the aging of the TMI sensor over the lifetime of the TRMM mission, could potentially have caused a drift in the TMI radiometric calibration.

This dissertation aims to eliminate systematic instrumental errors in TMI, so that changes in its measurements only reflect real changes in environmental conditions, and not orbital, diurnal, or long term variations in its radiometric calibration. A systematic brightness temperature bias, caused by an emissive main reflector antenna for TMI, is estimated 
using comparisons with a radiative transfer model (RTM), and a correction is applied based on orbit time and day of the year.

\section{$1.1 \quad$ TRMM Satellite Description}

TRMM was launched on November 1997, into a near circular, non-sun-synchronous orbit at $350 \mathrm{~km}$ altitude with an inclination of 35 degrees. This orbit provides extensive coverage in the tropics and allows each location to be covered at a different local time each day that cycles through a 24-hour period in approximately 46 days. Because of this low altitude, the satellite experienced significant atmospheric drag, which required frequent orbit "delta-velocity" burns of an on-board propulsion system to maintain this orbital altitude. At the end of the prime mission (August 2001), the satellite was boosted to an altitude of $402 \mathrm{~km}$ to increase its mission life, and the satellite is expected to remain in this orbit for at least several more years before it runs out of fuel and then de-orbits.

There are four principal rainfall remote sensing instruments on TRMM, namely: the TRMM Microwave Imager (TMI), the Precipitation Radar (PR), the Visible and Infrared Scanner (VIRS) and the Lightning Imaging sensor (LIS); however, this dissertation is concerned solely with the TMI, a conically scanning total power microwave radiometer, which is mounted on the upper deck of the TRMM satellite as shown in Fig. 1.1. The conical scan allows brightness temperature $(\mathrm{Tb})$ measurements to be obtained over an arc of 130 degrees in azimuth, which results in a wide measurement swath of $759 \mathrm{~km}$. The TMI design is a derivative of the Defense Meteorological Support Program's (DMSP) Special Sensor Microwave Imager (SSMI); a $10.7 \mathrm{GHz}$ pair of channels was added to SSM/I and the 22.235 
GHz channel was shifted to $21.4 \mathrm{GHz}$ (see Appendix-A for more details). The geophysical measurements derived from TMI include:

- atmospheric parameters: water vapor, cloud liquid water, and rainfall intensity

- oceanic surface parameters: wind speed and sea surface temperature (SST).

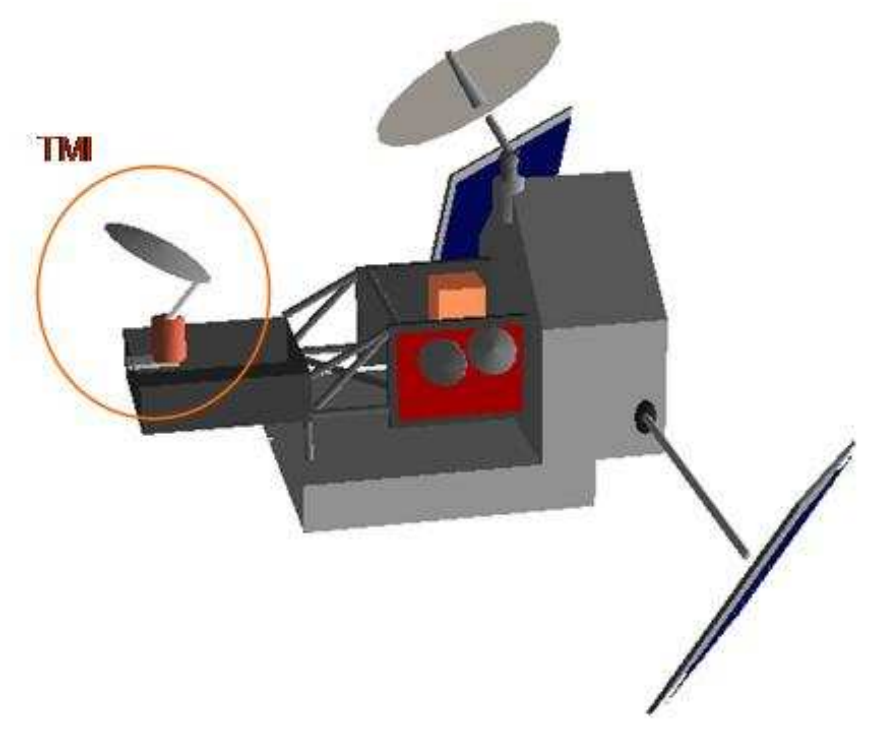

Figure 1.1: The TRMM Observatory.

\subsection{TMI Brightness Temperature Anomaly}

Shortly after the TRMM satellite launch, during the initial on-orbit instrument checkout, members of the TRMM science team discovered an anomaly in TMI brightness temperature measurements. After a thorough engineering evaluation, which included near simultaneous cross-calibration with the Special Sensor Microwave Imager (SSMI), it was concluded 
that the TMI brightness temperature measurements exhibited a consistent, warm bias in all channels compared to the pre-launch values. An analysis performed by Wentz et al. [7] modeled the slightly degraded observations by assuming a frequency independent radiometric bias, which could be explained by a reflector emissivity of $\sim 4 \%$ (reflectivity $96 \%$ ). This lead to a measured antenna brightness temperature of the form

$$
T_{a n t}=\Gamma T_{s c}+(1-\Gamma) T_{p h y}
$$

where

$T_{s c}$ is the apparent scene brightness temperature;

$\Gamma$ is the antenna surface power reflectivity $(96 \%)$;

$(1-\Gamma)$ is the reflector surface emissivity; and

$T_{p h y}$ is the reflector surface physical temperature.

The second term of (1.1) represents the "self blackbody emission" of the reflector, which was the source of the observed brightness temperature bias.

In the TRMM 1B11 (TMI brightness temperature) data product, a correction is made for this imperfect reflector by inverting this equation and solving for the apparent brightness temperature. Unfortunately, on TMI the reflector temperature is not measured; therefore only an estimate of the long-term ( $\sim 5$ month) orbit average (constant) temperature is used for the additive bias correction.

Wentz estimated the values of $\Gamma$ and $T_{p h y}$ using near-simultaneous collocations of TMI with SSMI brightness temperatures over the ocean (on DMSP satellites F-10, F-11 and F-13) 
between December 1997 and April 1998. A microwave radiative transfer model was used to normalize the SSMI brightness temperature observations for differences in frequencies and incidence angles (frequencies are identical except for the water vapor channel and the addition of the TMI $10.7 \mathrm{GHz}$ channels), and a linear regression was applied to all the relative biases between SSMI and TMI for this period. The slope of this regression provided an estimate of $\Gamma$, and the y-intercept of the regression provided an estimate of the mean value of $T_{p h y}$. The slopes and intercepts for each polarization are consistent for all the channels, as shown in Table 1.1.

Table 1.1: Slopes and Intercepts from SSMI inter-comparison from Wentz [7].

\begin{tabular}{|c|c|c|c|c|}
\hline \multirow{2}{*}{ Frequency } & \multicolumn{2}{|c|}{ Slope } & \multicolumn{2}{c|}{ Intercept } \\
\cline { 2 - 5 } & V Pol. & H Pol. & V Pol. & H Pol. \\
\hline $19.3 \mathrm{GHz}$ & -0.0370 & -0.0284 & 11.2 & 8.2 \\
\hline $21.3 \mathrm{GHz}$ & -0.0377 & - & 11.1 & - \\
\hline $37.0 \mathrm{GHz}$ & -0.0375 & -0.0274 & 11.1 & 8.1 \\
\hline $85.5 \mathrm{GHz}$ & -0.0396 & -0.0277 & 11.1 & 6.6 \\
\hline
\end{tabular}

The $\Gamma$ and $T_{p h y}$ estimates were verified during several orbits in January 1998 and again in September 1998, when the TRMM satellite was rotated in pitch by 180 degrees thereby causing the TMI antenna to view radiometrically cold space. The observed brightness temperature biases during the deep space observations were in good agreement with the $\mathrm{Tb}$ errors predicted using the $\Gamma$ and $T_{p h y}$ estimated from the SSMI cross-calibration.

\subsection{TMI Inter-satellite Calibration}

Recently, there has been an increased interest in the use of the TMI for inter-satellite radiometric calibration. Because TRMM flies in a non-sun-synchronous orbit, it has frequent near-simultaneous collocations with microwave imagers flown on polar (sun-synchronous) 
satellites. This provides the opportunity to remove systematic brightness temperature biases between different radiometers; thereby enabling the production of radiometrically consistent, multi-satellite, microwave time-series for weather and climate research [8]. This is particularly applicable to the future GPM mission, which will fly a core observatory satellite in a TRMM-like orbit with a GPM Microwave Imager (GMI), to be used as a radiometric cross-calibration transfer standard for the GPM constellation of independent microwave radiometers.

To this end, NASA's Precipitation Measurement Mission (PMM) Science Team's Inter-Calibration Working Group (ICWG) is performing research, using the TMI as a proxy for the GMI, to develop and compare radiometric cross-calibration techniques for the GPM mission. As part of this activity, TMI was inter-compared with SSMI and WindSat using near-simultaneous collocations over a period of one year after a RTM was used to normalize the frequency and incidence angle differences between different radiometers. It was observed that the relative biases between TMI and WindSat are dependent on the local time of the observation, with approximately $1 \mathrm{~K}-2 \mathrm{~K}$ difference between morning and evening. After extensive investigation, this time of day dependent variation in the relative calibration bias was attributed to changes in the TMI radiometric calibration associated with the reflector physical temperature over an orbit, which was due to solar heating over the daylight portion of the orbit and radiation cooling during the earth eclipse portion. 


\subsection{Dissertation Purpose and Organization}

The purpose of this dissertation is to provide a robust radiometric calibration for the TMI to correct systematic brightness temperature errors, which vary dynamically with orbit position (time) and day of the year. This dissertation investigates the presence of time-varying $\mathrm{Tb}$ radiometric calibration in TMI by inter-calibrating TMI observations with near-simultaneous collocations with WindSat and SSMI on DMSP satellites F-13 and F14. A microwave RTM is used to normalize the near-simultaneous WindSat and SSMI Tb observations over oceans to TMI frequencies and incidence angles. The dependence of the relative biases between TMI and WindSat Tb's on various environmental parameters (e.g., sea surface temperature, columnar water vapor) and physical parameters, (e.g., latitude and time of day) is analyzed in this dissertation.

The inter-calibration analysis described above confirmed the presence of a timevarying radiometric calibration error for TMI, which is manifested as a time of day dependent variation of the relative biases between TMI and both WindSat and SSMI Tb's. In this dissertation, we provide convincing evidence that these time-varying Tb biases in TMI are caused by variations in the physical temperature of the emissive TMI reflector antenna. The Tb bias exists in all the TMI frequencies and polarizations, and they result in significant errors in the TMI precipitation retrievals, as well as other atmospheric and surface geophysical parameter retrievals.

The goal of this dissertation is to provide an empirical correction that corrects this time-varying radiometric calibration error. Fortunately, the TMI biases can be estimated by comparing the brightness temperature observations for a single channel $(10.7 \mathrm{GHz} \mathrm{V}$ - 
polarization) with RTM Tb predictions,. The estimated Tb corrections are applied to all channels as a function of orbit time, which is different for every day of the one year period. Furthermore, this dissertation provides a qualitative physical basis for the observed $\mathrm{Tb}$ orbital bias patterns and provides conclusive evidence that the empirical correction applied to TMI Tb measurements (independent of both ocean and land) adequately corrects the time-varying TMI calibration. This is accomplished by demonstrating that the local timeof-day dependence (in the uncorrected TMI Tb values) is removed in the corrected TMI Tb's.

In addition, this dissertation also aims to serve as a template for future inter-satellite radiometric calibration efforts at the Central Florida Remote Sensing Laboratory. A technique similar to the one described in this dissertation is currently being used to calibrate the QuikScat Radiometer. The analysis performed in this dissertation can also be repeated for radiometers in the GPM mission, such as the MADRAS radiometer on the Megha-Tropiques mission, which has a non-sun-synchronous tropical orbit similar to the TRMM orbit. Such analysis can detect the presence of systematic biases in the radiometers that are a part of the GPM mission.

This dissertation is organized as follows: Chapter 2 discusses the discovery of the TMI time-varying radiometric calibration and presents a hypothesis for its cause; Chapter 3 describes the development of an empirical correction for the TMI instantaneous brightness temperature biases (time-variable radiometric calibration), which are modeled as functions of orbit time and day of year; Chapter 4 summarizes this dissertation and presents conclusions and also presents a TMI Level-1 science data processing algorithm that applies a real-time 
radiometric calibration bias correction suitable for the upcoming version-7 (V7) TRMM 1B11 data product. 


\section{CROSS-CALIBRATION OF TMI, WINDSAT AND SSMI}

This chapter provides evidence of a time-varying radiometric calibration error for TMI by presenting the results of the cross-calibration of TMI with SSMI and WindSat. The intersatellite calibration technique that confirmed the presence of this time-varying TMI bias was developed at the CFRSL by Hong [8-11] and improved under this dissertation during research activities associated with the ICWG [12-16]. This technique uses a radiative transfer model to predict expected $\mathrm{Tb}$ differences between a pair of radiometer channels that simultaneously view a homogeneous environmental scene. Given knowledge of the atmospheric and oceanic environmental parameters, the RTM accounts for the expected change in $\mathrm{Tb}$ at the respective frequencies and incidence angles of the two radiometers under comparison. Afterwards, one compares the measurements and then computes the unexplained relative radiometric bias between the channels, which should be independent of environmental conditions.

Under this dissertation research, this approach was applied in 2007 to compare collocated oceanic brightness temperature measurements between TMI, WindSat, and SSMI on DMSP F-13 and F-14. Among these radiometers, TMI has a low-inclination earth orbit, while WindSat and SSMI have near-polar sun-synchronous orbits. For this radiometer crosscalibration, the Sun Synchronous observations were normalized to the TMI frequencies and incidence angles, after which the radiometric biases between TMI and the Sun Synchronous radiometers were calculated.

Section 2.2 describes the common ICWG dataset; Section 2.3 explains the procedure used to generate near-simultaneous match-ups between TMI and the polar orbiting radiometers; Section 2.4 describes the construction of the RTM; and Section 2.5 describes 
the inter-calibration methodology and the results obtained, which confirmed the time-varying nature of the TMI radiometric calibration.

\subsection{Frequency and Incidence Angle Normalization Using Taylor Series}

Hong [10, 11] developed an inter-satellite microwave radiometric cross-calibration technique to estimate brightness temperature measurement biases between a pair of radiometer channels operating at slightly different frequencies and incidence angles. In this approach predictions were made of Tb's at a destination (target) frequency from Tb's of a close by source frequency by expansion of the brightness temperature spectrum in a Taylor series centered at the source frequency. Under a given set of geophysical conditions, the observed brightness temperature of a radiometer is determined by its frequency and its incidence angle. The relation between the brightness temperature and frequency can be obtained for a fixed incidence angle through radiative transfer modeling of the brightness temperature and expansion as a polynomial function of frequency. Fig. 2.1 shows an example of the modeled $\mathrm{Tb}$ spectrum for an incidence angle of $53.2^{\circ}$. 


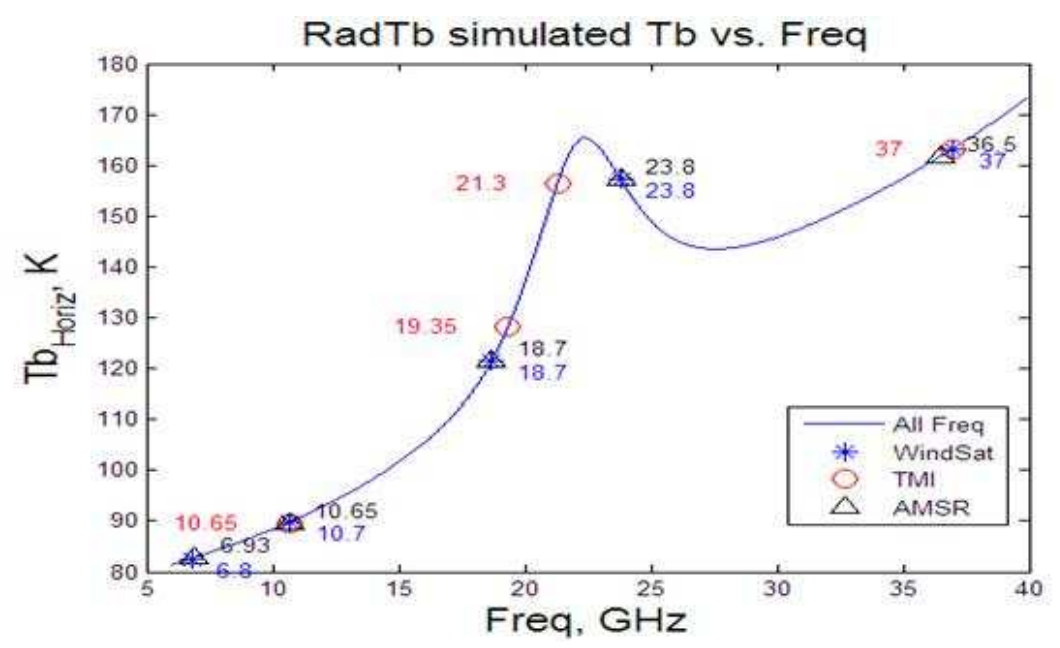

Figure 2.1: Tb spectrum example from Hong [10].

According to Hong [10], the Taylor series expansion of $\mathrm{Tb}$ as a function of frequency about a frequency f0 can be expressed as:

$$
\begin{aligned}
T_{b}\left(f_{1}\right) & =T_{b}\left(f_{0}\right)+T_{b}^{\prime}\left(f_{0}\right)\left(f_{1}-f_{0}\right)+T_{b}^{\prime \prime}\left(f_{0}\right) \frac{\left(f_{1}-f_{0}\right)^{2}}{2 !}+\ldots+T_{b}^{(n)}\left(f_{0}\right) \frac{\left(f_{1}-f_{0}\right)^{n}}{n !}+. \\
T_{b}^{(n)} & =\left.\frac{\partial^{n} T_{b}(f)}{\partial f^{n}}\right|_{f=f_{0}}
\end{aligned}
$$

where

$f_{0}$ is the frequency of the source channel

$f_{1}$ is frequency of the target channel

$T_{b}(f)$ is the brightness temperature as a function of frequency, and

$T_{b}^{(n)}$ is the nth derivative of the $\mathrm{Tb}(\mathrm{f})$ function with respect to frequency evaluated at the source frequency. 
The $\mathrm{Tb}$ is also modeled as a linear function of incidence angle, and the transformation is expressed as:

$$
T b\left(\theta_{1}\right)=T b\left(\theta_{0}\right)+k(\theta) *\left(\theta-\theta_{0}\right)
$$

where

$$
k(\theta)=\left.\frac{\partial T_{b}(\theta)}{\partial \theta}\right|_{\theta=\theta_{0}}
$$

where

$\theta_{0}$ is the incidence angle of the source channel

$\theta_{1}$ is incidence angle of the target channel, and

$k(\theta)$ is the partial derivative of $\mathrm{Tb}$ with respect to incidence angle derived from the RTM evaluated at the target frequency as a function of the corresponding environmental parameters.

The procedure developed during this dissertation research is a simplified version of the Taylor series method. The Taylor series expansion provides a unified normalization function for radiometer channel pairs with any frequency and incidence angle combination for a given set of environmental conditions; however, for a specific pair of radiometer channels, the Taylor series normalization is simply an additive correction for both frequency and incidence angle. Thus, the $\mathrm{Tb}$ measurements for pair-wise comparisons are normalized using the predicted difference between the two radiometer channels $(\Delta)$ as follows: 


$$
\begin{aligned}
& \Delta=T b_{R T M}\left(f_{1}, \theta_{1}\right)-T b_{R T M}\left(f_{0}, \theta_{0}\right) \\
& T b_{\text {norm }}\left(f_{1}, \theta_{1}\right)=T b_{\text {meas }}\left(f_{0}, \theta_{0}\right)+\Delta
\end{aligned}
$$

where

$f_{0}$ is the source channel center frequency

$\theta_{0}$ the source channel incidence angle

$f_{1}$ is the target channel frequency

$\theta_{1}$ is the target channel incidence angle

$T b_{R T M}$ is the brightness temperature predicted by the RTM for the given frequency and incidence angle

$T b_{\text {norm }}\left(f_{1}, \theta_{1}\right)$ is the normalized source channel brightness temperature and $T b_{\text {meas }}\left(f_{0}, \theta_{0}\right)$ is the measured source channel brightness temperature.

This process takes the effect of geophysical parameters on the Tb normalization into account, as the RTM Tb's are calculated for the geophysical parameters for every match-up between the given pair of radiometers.

\subsection{ICWG Common Dataset}

The Level 1C brightness temperature product (provided by Colorado State University [17]) is designed to be a prototype for GPM, which will have an inter-calibrated dataset 
featuring multiple radiometers. The common dataset used in the ICWG evaluation consists of data from TMI, SSMI on DMSP F-13 and F-14 satellites and WindSat for the time period July 2005 to June 2006. Level 1C files are stored in the Hierarchical Data Format (HDF) and are organized such that each file contains one orbit of data from the start of the ascending pass (i.e. from the southernmost latitude) till the end of the descending pass. The L-1C input data is obtained from the standard products for each radiometer, i.e., TRMM 1B11 (version 6) for TMI [18], the Temperature Data Record (TDR) for SSMI [19] and the Sensor Data Record (SDR) for WindSat [20].

The ICWG uses the NOAA global numerical weather model Global Data Assimilation System (GDAS) [21-22], which is provided every 6 hours (0000, 0600, 1200 and 1800 GMT) on a $100 \mathrm{~km}$ grid, to obtain environmental data like sea surface temperature (SST), wind speed and direction, and atmospheric temperature, pressure, humidity and cloud liquid water profiles. The atmospheric data from GDAS is provided at 21 constant pressure layers between $1000 \mathrm{mb}$ and $100 \mathrm{mb}$, and the GDAS files also provide the geopotential heights of each layer.

The next section explains how the data from these different radiometers and GDAS are combined to generate the match-up data set used for radiometric inter-calibration.

\subsection{Generation of Near-Simultaneous Collocations}

The inter-calibration study has been performed by using more than 100,000 spatial/temporal collocations of TMI radiometer Tb's with WindSat/SSMI brightness temperatures using Level 1C data during the year July 2005 - June 2006, which have been merged 
with environmental data from GDAS. The simplified block diagram shown in Figure 2.2 illustrates the process of generating these match-ups.



Figure 2.2: Generation of Match-up Dataset.

The match-up files are ASCII files containing Tb measurements, incidence and azimuth angle information, and environmental parameters as shown in the figure above. The match-ups are generated with a maximum temporal tolerance of 1 hour between satellite measurements and spatial quantization of 1 degree (latitude by longitude boxes). Three collocation files (TMI-WindSat, TMI-F13 and TMI-F-14) are generated per day. The TMIWindSat collocation file contains 128 values for every 1 degree collocation, as shown in Table 2.1. The TMI-SSMI collocation file contains 118 values for every 1 degree collocation, as shown in Table 2.2. One significant difference in the two collocation formats is that the WindSat collocations contain the instantaneous incidence angles for each WindSat channel 
(as these are different from one another), while the SSMI collocations contain one incidence angle for all the SSMI channels.

Table 2.1: Composition of TMI - WindSat collocation files.

\begin{tabular}{|c|c|}
\hline Col. number & Parameter \\
\hline 1 & TMI ascending/descending flag \\
\hline 2 & WindSat ascending/descending flag \\
\hline 3 & Latitude \\
\hline 4 & Longitude \\
\hline 5 & TMI time (min from start of day) \\
\hline 6 & WindSat time (min from start of day) \\
\hline 7 & TMI incidence angle (deg) \\
\hline $8-10$ & TMI azimuth (min,mean,max) (deg) \\
\hline $11-19$ & TMI Mean Tb's (K) \\
\hline $20-28$ & TMI Tb Std. Dev. (K) \\
\hline 29 & Number of TMI samples \\
\hline $30-34$ & WindSat incidence angle (deg) \\
\hline $35-37$ & WindSat azimuth (min,mean,max) (deg) \\
\hline $38-47$ & WindSat Mean Tb's (K) \\
\hline $48-57$ & WindSat Tb Std. Dev. \\
\hline 58 & Number of WindSat samples \\
\hline 59 & GDAS surface pressure (mb) \\
\hline 60 & GDAS surface temperature (K) \\
\hline 61 & GDAS $2 \mathrm{~m}$ temperature $(\mathrm{K})$ \\
\hline 62 & GDAS wind $-\mathrm{u}(\mathrm{m} / \mathrm{s})$ \\
\hline 63 & GDAS wind $-\mathrm{v}(\mathrm{m} / \mathrm{s})$ \\
\hline $64-84$ & GDAS temperature profile (K) \\
\hline $85-105$ & GDAS relative humidity profile \\
\hline $106-126$ & GDAS geopotential height $(\mathrm{m})$ \\
\hline 127 & Salinity \\
\hline 128 & REMSS CLW retrieval (mm) \\
\hline
\end{tabular}


Table 2.2: Composition of TMI - SSMI collocation files.

\begin{tabular}{|c|c|}
\hline Col. number & Parameter \\
\hline 1 & TMI ascending/descending flag \\
\hline 2 & WindSat ascending/descending flag \\
\hline 3 & Latitude \\
\hline 4 & Longitude \\
\hline 5 & TMI time (min from start of day) \\
\hline 6 & SSMI time (min from start of day) \\
\hline 7 & TMI incidence angle (deg) \\
\hline $8-10$ & TMI azimuth (min,mean,max) (deg) \\
\hline $11-19$ & TMI Mean Tb's (K) \\
\hline $20-28$ & TMI Tb Std. Dev. (K) \\
\hline 29 & Number of TMI samples \\
\hline 30 & SSMI incidence angle (deg) \\
\hline $31-33$ & SSMI azimuth (min,mean, $\max )(\mathrm{deg})$ \\
\hline $34-40$ & SSMI Mean Tb's (K) \\
\hline $41-47$ & SSMI Tb Std. Dev. \\
\hline 48 & Number of SSMI samples \\
\hline 49 & GDAS surface pressure (mb) \\
\hline 50 & GDAS surface temperature (K) \\
\hline 51 & GDAS $2 \mathrm{~m}$ temperature $(\mathrm{K})$ \\
\hline 52 & GDAS wind $-\mathrm{u}(\mathrm{m} / \mathrm{s})$ \\
\hline 53 & GDAS wind-v $(\mathrm{m} / \mathrm{s})$ \\
\hline $54-74$ & GDAS temperature profile $(\mathrm{K})$ \\
\hline $75-95$ & GDAS relative humidity profile \\
\hline $96-116$ & GDAS geopotential height $(\mathrm{m})$ \\
\hline 117 & Salinity \\
\hline 118 & REMSS CLW retrieval $(\mathrm{mm})$ \\
\hline
\end{tabular}




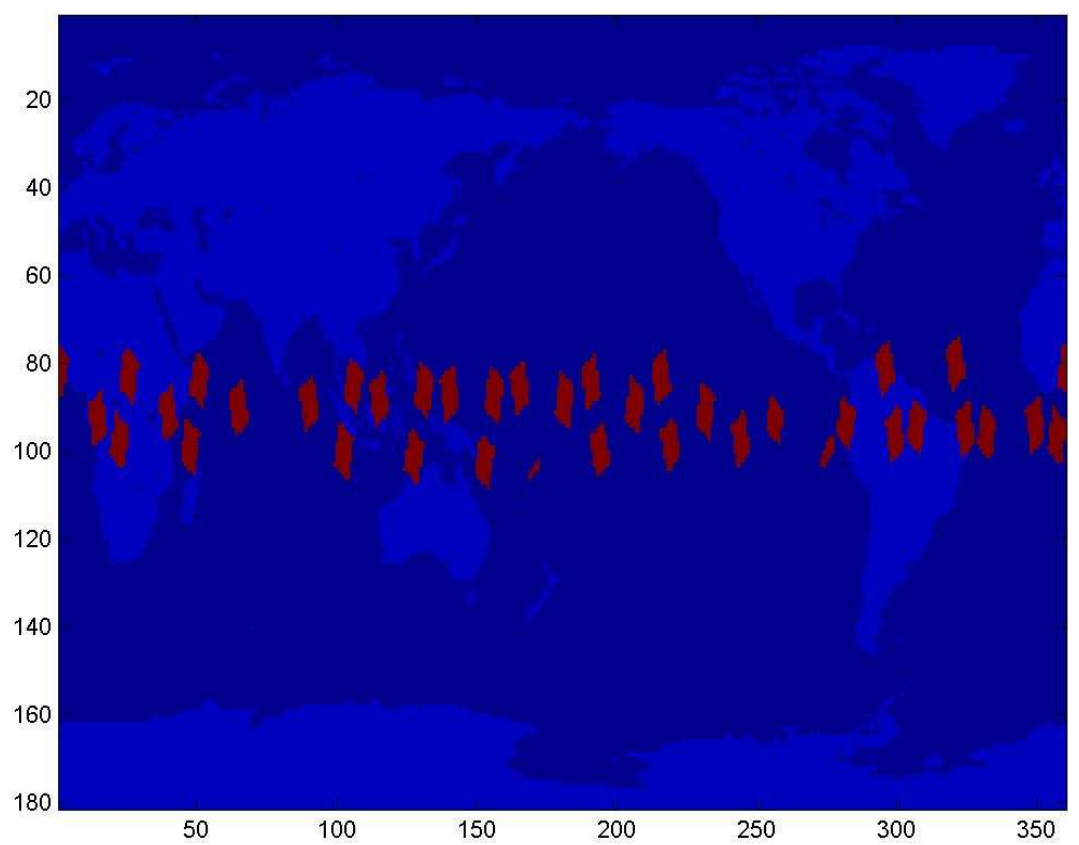

(a) July 1, 2005.



(b) July 13, 2005.

Figure 2.3: Geo-location of TMI-WindSat collocations. 


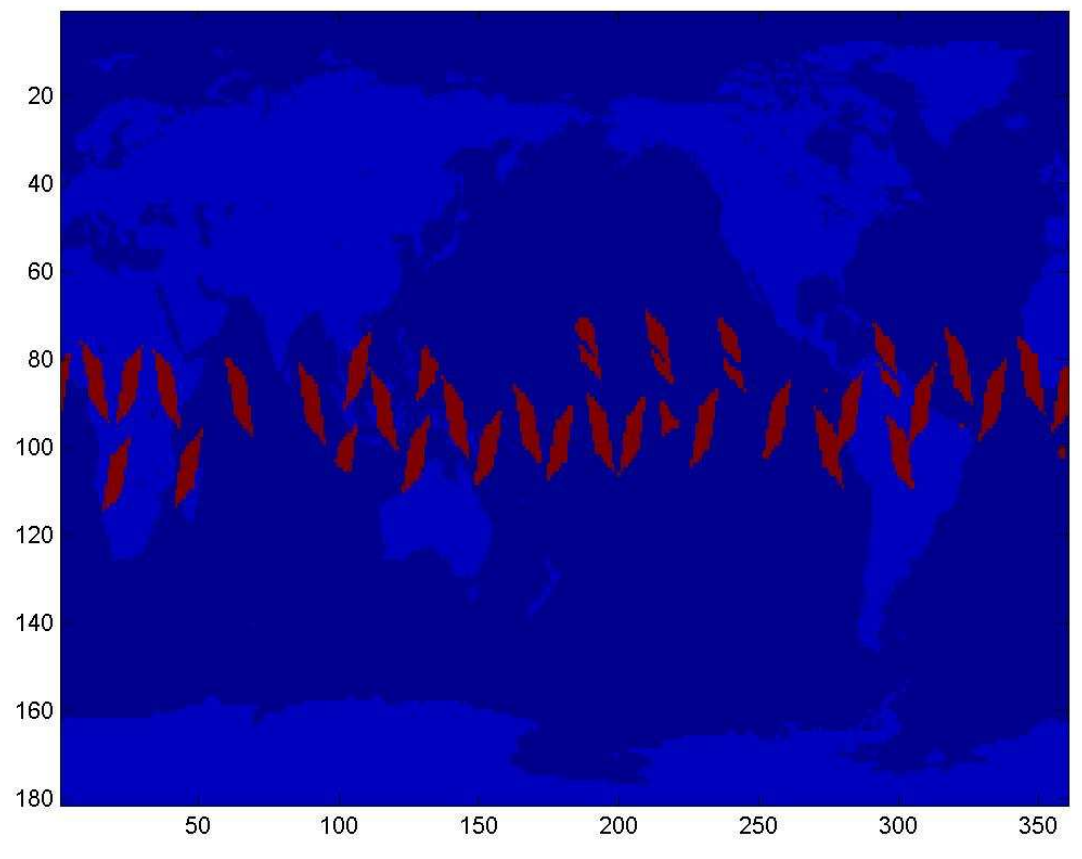

(c) July 24, 2005 .

Figure 2.3: Geo-location of TMI-WindSat collocations.

The location of the collocations varies by latitude on a $\sim 23$ day cycle (the half-period of the TRMM). For example, the collocations between TMI and WindSat all lie close to the equator on July 1, 2005, as shown in Fig. 2.3 panel-(a). The continental land masses are depicted in light blue while the collocations are highlighted in red. The collocations then move gradually towards the outer latitudes, till they reach the outer edge of TRMM swath at $\pm 40^{\circ}$ latitudes. This is shown in Fig. 2.3 panel-(b) which shows the TMI WindSat collocations for July 13, 2005. After this, the collocations move back towards the equator till the TRMM orbital half-cycle is completed. Panel-(c) shows the TMI-WindSat collocations for July 24, 2005, for which case the collocations are again located close to the equatorial line. Due to the similar equator crossing time of WindSat, SSMI F-13 and SSMI F-14, the 
collocations for TMI and SSMI F-13 and F-14 on July 1,2005 are similar to TMI/WindSat, as shown in Fig. 2.4.



(a) TMI - SSMI F-13.

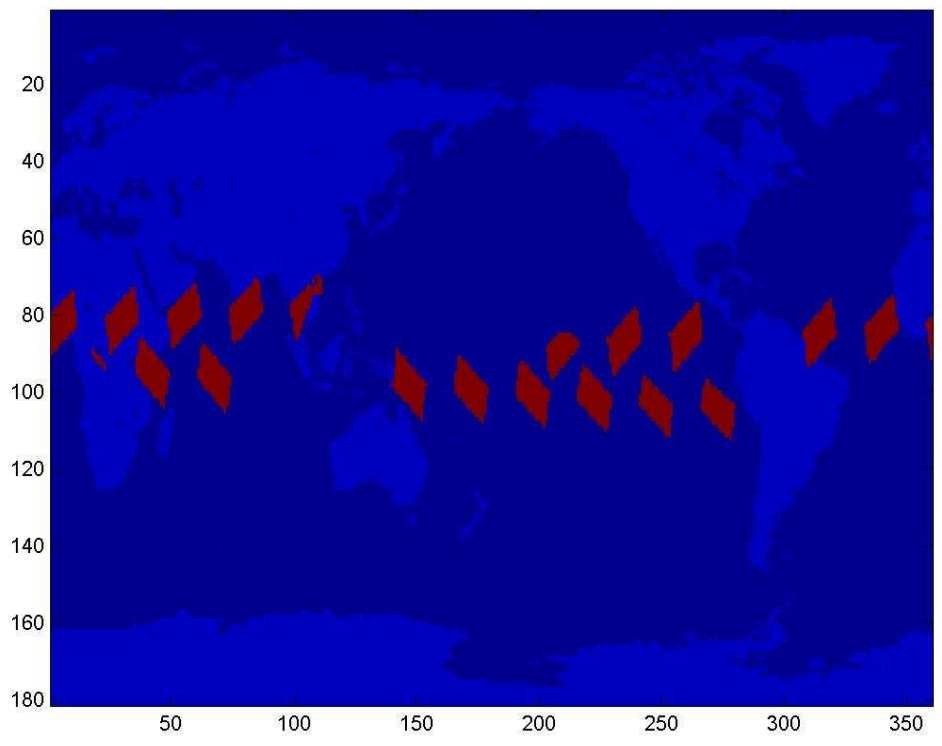

(b) TMI-SSMI F-14

Figure 2.4: Geo-location of TMI - SSMI collocations. 
Before generating the match-up files, the radiometer data is earth gridded into one degree bins. All samples in a one degree bin are averaged, and the mean and std. deviation of Tb's for each bin are computed and stored. The match-up records also specify if the samples are from an ascending pass or a descending pass and also provide the instantaneous incidence angles for both radiometers. The match-up database contains nearly half a million sets of $1^{\circ}$ collocations (over both land and oceans), with each record containing all the relevant information about the collocation scene. The large size of the database is extremely advantageous for the inter-satellite calibration analysis, because the data can easily be stratified by latitude, time or various environmental parameters such as SST, water vapor, wind speeds or cloud liquid water. In contrast, the analysis performed in Hong [10] was based on weekly averages from 6 discontinuous weeks of data. The large number of collocations generated as part of this dissertation allowed analysis of fine bins of environmental parameters (i.e. $2 \mathrm{~K}$ bins for SST); while such analysis could not be performed in Hong due to the smaller number of collocation samples.

Previously, the collocations between TMI and WindSat provided by Hong [10] were generated on a pixel by pixel basis. For every orbit of TMI and WindSat that overlapped in time, the data in every $5^{\circ} \times 5^{\circ}$ box was compared to check for the existence of both TMI and WindSat samples. For every box that had such samples, every pair of TMI and WindSat pixels were compared to check for the temporal and spatial difference between the two pixels, and the pixel pairs with spatial and temporal differences within specified thresholds were added to the collocation database. 
This dissertation uses a modified procedure to generate collocations between TMI and WindSat/SSMI. The collocations are generated using mean Tb's for 1 degree earth-gridded boxes, instead of pixel pairs. Earth gridding the data in 1 degree bins before searching for near-simultaneous collocations reduces the processing time enormously. For example, searching for near-simultaneous collocations between one orbit of TMI and WindSat on a pixel by pixel basis would require approximately 80 billion comparisons. Hong [10] improves the performance by an order of magnitude by down-sampling the data to $5^{\circ} \times 5^{\circ}$ bins and looking for TMI and WindSat incidences in those bins. Generating pixel-wise collocations in a fully populated $5^{\circ} \times 5^{\circ}$ bin would typically require approximately 3 million comparisons. The procedure used in this dissertation further reduces the processing time by another order of magnitude, as searching for near-simultaneous collocations between $1^{\circ}$ earth-gridded orbits of TMI and WindSat requires only 65,000 comparisons.

While the current procedure offers a significant improvement in processing time, the collocation outputs are more coarsely quantized spatially when compared to pixel by pixel match-ups; however, this reduction in spatial quantization does not adversely affect the inter-calibration results. The $\mathrm{Tb}$ mean of each $1^{\circ}$ box is obtained by averaging more than 50 pixel observations, which cancels the radiometer $N E \Delta T$ random noise present in individual pixel observations, and thus, provides a more accurate estimate of the $\mathrm{Tb}$ of the given scene.

Hong performed analysis using weekly mean biases between TMI and various sunsynchronous radiometers. While such an approach is useful for detecting constant calibration biases between radiometers, it is not suitable for the detection of time-varying biases in radiometers at periods less than one week. For this dissertation, the availability of a large 
number of $1^{\circ}$ boxes that were processed continuously to form a one-year time series, allowed us to discover the time-variable radiometric calibration of TMI. Further, since the correction of TMI's time-varying calibration is the goal of this dissertation, we performed our analysis at 5 minute intervals utilizing this large database of $1^{\circ}$ boxes, which was the direct result of the improved processing time achieved during this dissertation research effort.

The match-up files also contain atmospheric parameters from GDAS, including the atmospheric profiles of pressure, temperature and water vapor at 21 levels as well as sea surface temperature and ocean wind speed and direction at $10 \mathrm{~m}$ height. Salinity values are obtained from monthly averages from National Oceanographic Data Center World Ocean Atlas (NODC WOA 1998) salinity. Due to the highly transient nature of clouds, it is desirable to obtain cloud liquid water values with a smaller temporal difference with the match-ups; therefore, columnar cloud liquid water is downloaded from the Remote Sensing Systems (REMSS) [23-24] TMI retrievals. Since the REMSS retrievals coincide with the match-ups, these cloud liquid water values are considered more appropriate than the GDAS predictions.

These match-up samples are then filtered to remove Tb outliers [25]. This is an important feature of the inter-calibration philosophy of "quality over quantity" i.e., it is better to have fewer higher quality points to establish the calibration biases than many lesser quality points. In each one degree bin, Tb samples with a standard deviation of more than $2 \mathrm{~K}$ in the vertical polarization and more than $3 \mathrm{~K}$ in the horizontal polarization are removed; as such high standard deviations are indicative of non-homogeneous environmental conditions, including rain contamination. Further editing based upon upper limits of brightness tem- 
peratures expected from a rain-free ocean observation is applied for all frequencies. Tables 2.3 and 2.4 show the limits for each of TMI (SSMI) and WindSat channels. The limits for the SSMI channels are identical to those of the corresponding TMI channels.

A conservative land mask is also applied to filter out land pixels. An image of the collocation regions shown in Fig. 2.5 illustrates the effect of the filtering process. Panel-(a) shows the TMI 37V Tb for the collocated 1 degree boxes without any filtering, where the Tb's have high values over land (land masses are marked with light blue) and lower values over the ocean. Panel-(b) shows the effect of applying the upper thresholds for the TMI and WindSat Tb's, in which the collocations over land are now removed. For collocations over rainy scenes, the standard deviation threshold removes these regions and leaves only observations over homogeneous rain-free ocean scenes, as shown in panel-(c).

Table 2.3: Upper limits for TMI (SSMI) channels for oceanic scenes.

\begin{tabular}{|c|c|c|c|c|c|c|c|}
\hline TMI Channels & $10 \mathrm{~V}$ & $10 \mathrm{H}$ & $19 \mathrm{~V}$ & $19 \mathrm{H}$ & $21 \mathrm{~V}$ & $37 \mathrm{~V}$ & $37 \mathrm{H}$ \\
\hline Max Tb $(\mathrm{K})$ & 185 & 115 & 230 & 200 & 260 & 240 & 210 \\
\hline
\end{tabular}

Table 2.4: Upper limits for WindSat channels for oceanic scenes.

\begin{tabular}{|c|c|c|c|c|c|c|c|c|c|c|}
\hline WS Channels & $6 \mathrm{~V}$ & $6 \mathrm{H}$ & $10 \mathrm{~V}$ & $10 \mathrm{H}$ & $18 \mathrm{~V}$ & $18 \mathrm{H}$ & $23 \mathrm{~V}$ & $23 \mathrm{H}$ & $37 \mathrm{~V}$ & $37 \mathrm{H}$ \\
\hline Max Tb $(\mathrm{K})$ & 200 & 120 & 200 & 150 & 250 & 200 & 260 & 230 & 250 & 200 \\
\hline
\end{tabular}






(a) Unfiltered collocations.

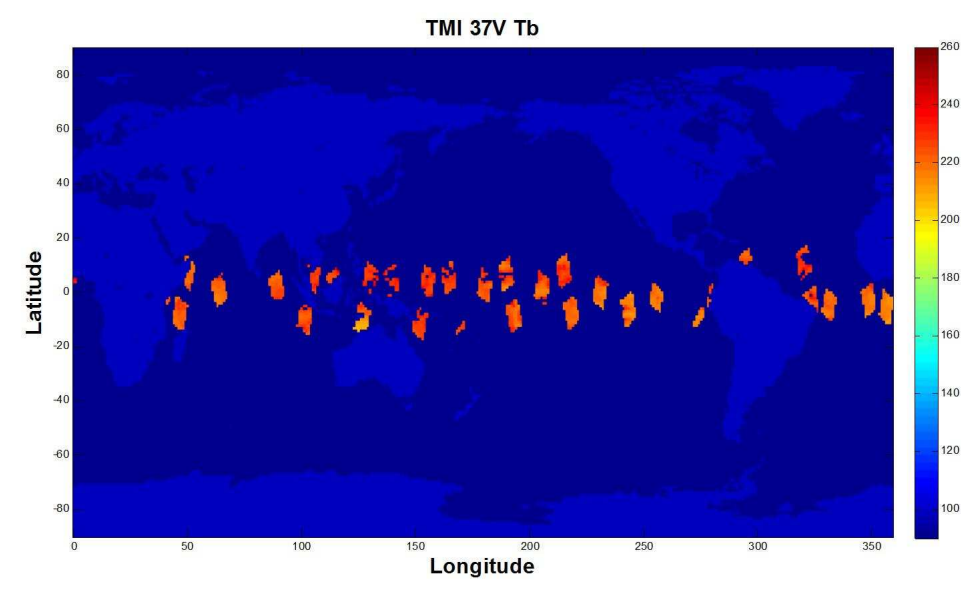

(b) Upper Tb thresholds applied.

Figure 2.5: Effect of the filtering process on TMI-WindSat collocations. 


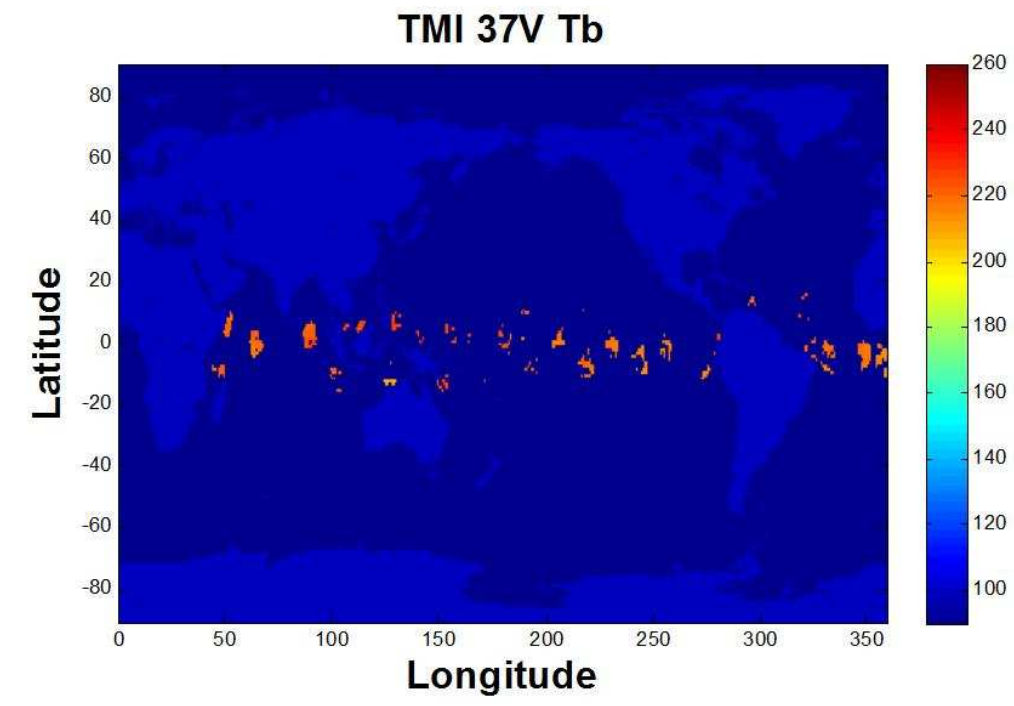

(c) After complete filtering.

Figure 2.5: Effect of the filtering process on TMI-WindSat collocations. 


\subsection{Radiative Transfer Modeling}

The inter-calibration method described in this chapter uses the ICWG radiative transfer model to account for frequency and incidence angle differences between two radiometers. An in-depth discussion of radiative transfer theory can be found in Ulaby, Moore and Fung [26] and Elachi [27]. This RTM uses the Elsaesser model [28] for ocean isotropic emissivity. The Elsaesser emissivity model requires sea surface temperature (SST), wind speed (WS), salinity, frequency and incidence angle as inputs. It calculates the isotropic ocean surface emissivity and ignores small wind direction effects, which were studied and found to have negligible effect on the relative biases. These anisotropic wind direction effects are not described in this dissertation. Figure 2.6 shows the variation of the $\mathrm{V}$ and $\mathrm{H}$ polarized surface emissions at $10.7 \mathrm{GHz}$ with wind speed, for an incidence angle of 53.4 degrees, SST $=300 \mathrm{~K}$ and salinity of $30 \mathrm{ppt}$. The H-pol emissivity increases from 0.25 for $\mathrm{WS}=0$ to 0.3 for WS $=15$, which is equivalent to a change in brightness of

$$
\Delta T_{b}=\Delta \epsilon * S S T \simeq 15 K
$$

and a sensitivity to wind speed of about $1 \mathrm{~K} /(\mathrm{m} / \mathrm{s})$ for the $10 \mathrm{H}$ channel. On the other hand, the V-pol emissivity increases less than 0.01 for the same increment of wind speed, which results in a sensitivity of $\sim 0.2 \mathrm{~K} /(\mathrm{m} / \mathrm{s})$.

Fig. 2.7 shows the surface emission of the ocean surface for low wind speed $(5 \mathrm{~m} / \mathrm{s})$. The Tb sensitivity at $10.7 \mathrm{GHz}$ is low for low SSTs; at $275 \mathrm{~K}$ the sensitivity is $0.2 \mathrm{~K} / \mathrm{K} \_\mathrm{SST}$ for V-pol and 0.025 K/K_SST for H-pol. The surface emissions are more sensitive at medium 
SSTs; at $290 \mathrm{~K}$ the sensitivity is $0.45 \mathrm{~K} / \mathrm{K} \_S S T$ for V-pol and $0.21 \mathrm{~K} / \mathrm{K} \_S S T$ for H-pol. The Tb sensitivity increases further for high SSTs; at $305 \mathrm{~K}$ the sensitivity is $0.65 \mathrm{~K} / \mathrm{K} \_\mathrm{SST}$ for V-pol and 0.3 K/K_SST for H-pol.



(a) V-pol

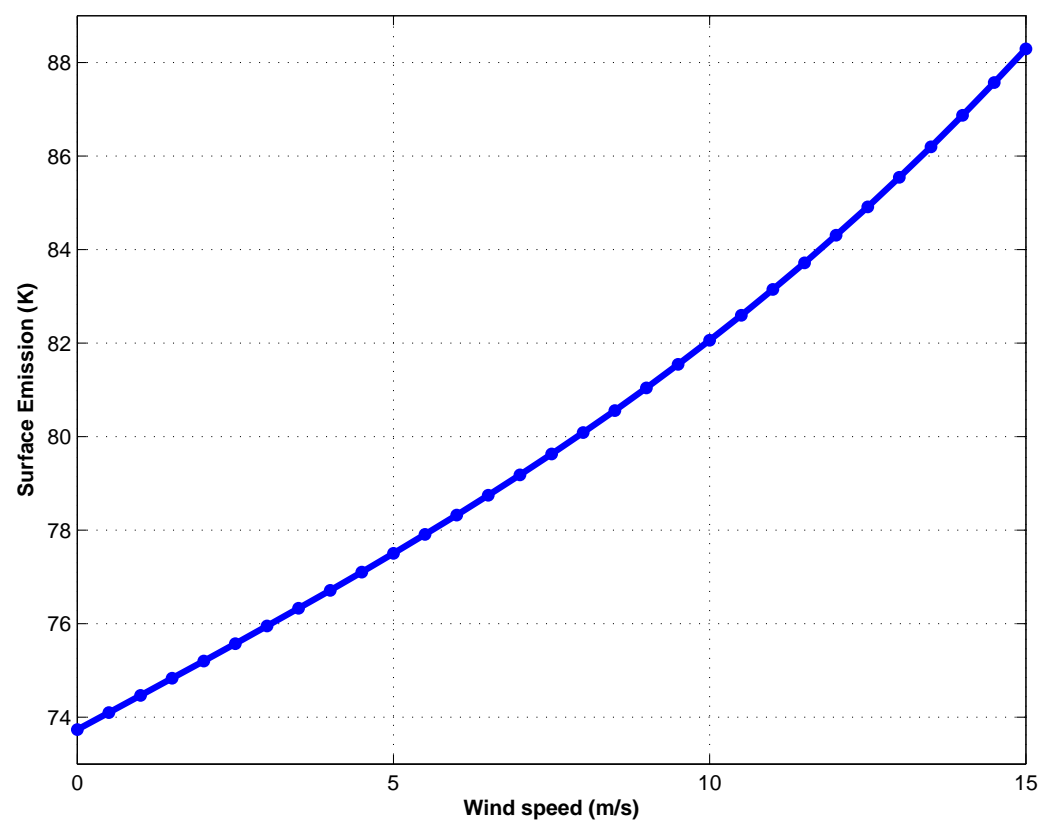

(b) H-pol

Figure 2.6: Variation of $10.7 \mathrm{GHz}$ surface emission with wind speed. 




(a) V-pol



(b) H-pol

Figure 2.7: Variation of $10.7 \mathrm{GHz}$ surface emission with SST. 
The Rosenkranz models for water vapor, cloud liquid water, oxygen and nitrogen absorption in the atmosphere [29] are used to calculate the atmospheric absorption coefficients. The largest contribution to the atmospheric absorption in the $18 \mathrm{GHz}-37 \mathrm{GHz}$ frequency range comes from water vapor. Fig. 2.8 shows the frequency variation of the water vapor absorption coefficient for different water vapor densities. The absorption coefficient at 22 GHz for a surface WV density of $10 \mathrm{~g} / \mathrm{m}^{3}$, which typically corresponds to a columnar density of about $25 \mathrm{~mm}$ (the typical value of columnar WV in our dataset, as shown in Fig. 3.8), is approximately 0.07 nepers $/ \mathrm{km}$. The absorption is low for the low frequencies, peaks at 22.3 $\mathrm{GHz}$ and drops again before rising monotonically for frequencies higher than $33 \mathrm{GHz}$. The $22.3 \mathrm{GHz}$ peak absorption values are almost directly proportional to the water vapor density. The cloud liquid water absorption coefficients, shown in Fig. 2.9, rise monotonically with frequency. The absorption coefficient The absorption coefficients rise faster with frequency for higher cloud liquid water densities. A cloud liquid water density of $0.01 \mathrm{~g} / \mathrm{m}^{3}$ corresponds to a columnar CLW of $0.01 \mathrm{~mm}$ (the typical value of CLW in our dataset, as shown in Fig. 3.8), since the thickness of the cloud is assumed to be approximately $1 \mathrm{~km}$. The absorption caused by $\mathrm{N} 2$ and $\mathrm{O} 2$ for frequencies lower than $37 \mathrm{GHz}$ is significantly lower than water vapor and cloud liquid water absorption $(<0.001$ nepers $/ \mathrm{km})$, as seen in Figures 2.10 and 2.11. There is an extremely strong $\mathrm{O} 2$ absorption signal at $60 \mathrm{GHz}$ which does not affect the inter-calibration analysis, as the radiometers in this dataset do not contain any channels close to that frequency. Thus, water vapor and CLW are the two atmospheric parameters that significantly affect X-band to Ka band frequency emissions. Thus, over the range of frequencies of interest, these two parameters, along with SST and wind speed are the four geophysical parameters that significantly impact the RTM modeled Tb. 


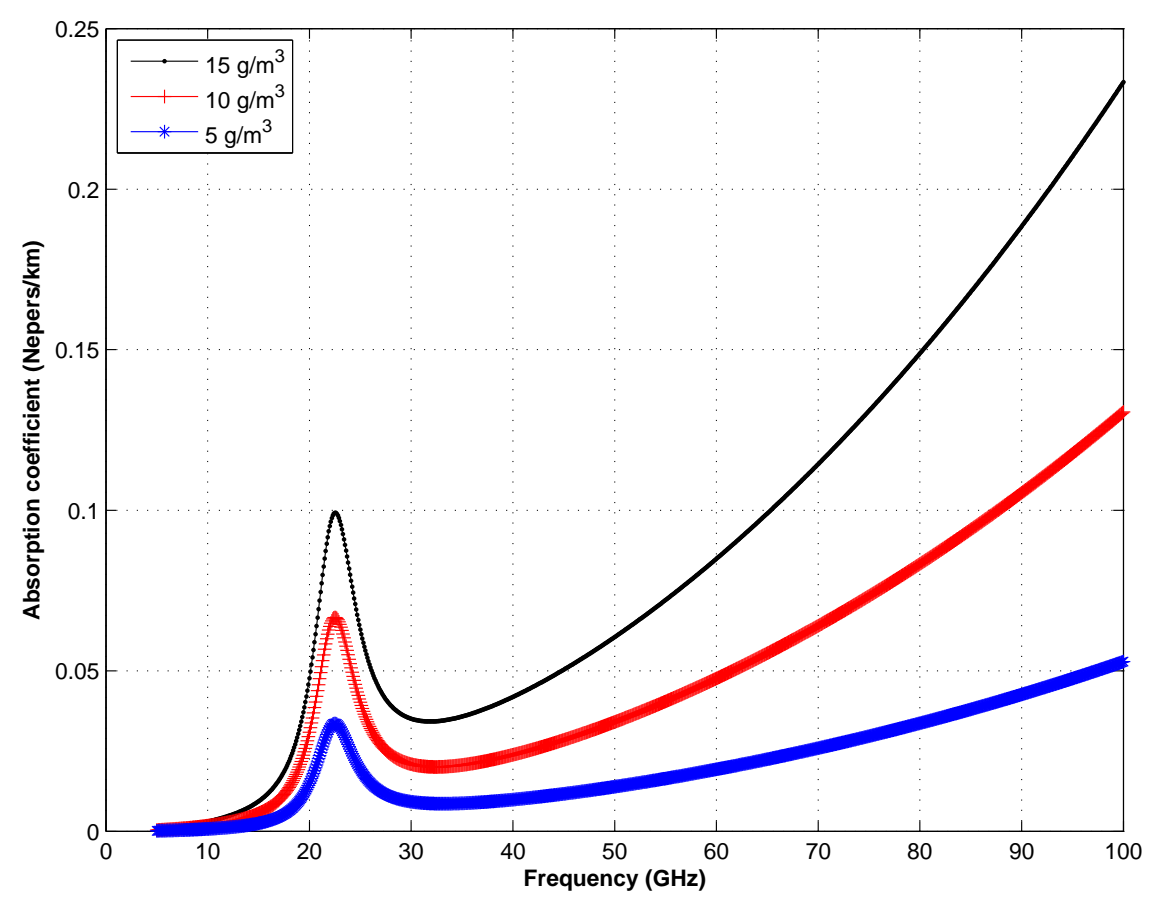

Figure 2.8: Frequency spectrum of WV absorption.

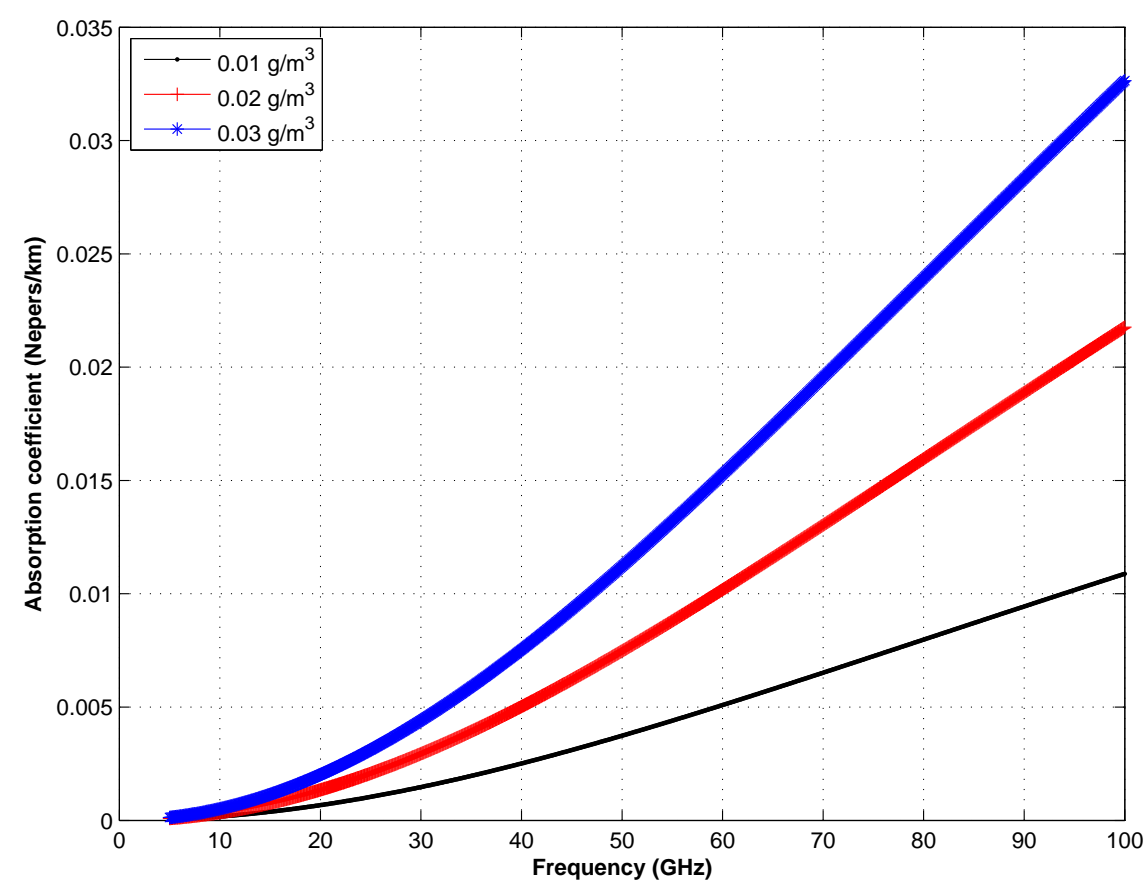

Figure 2.9: Frequency spectrum of CLW absorption. 


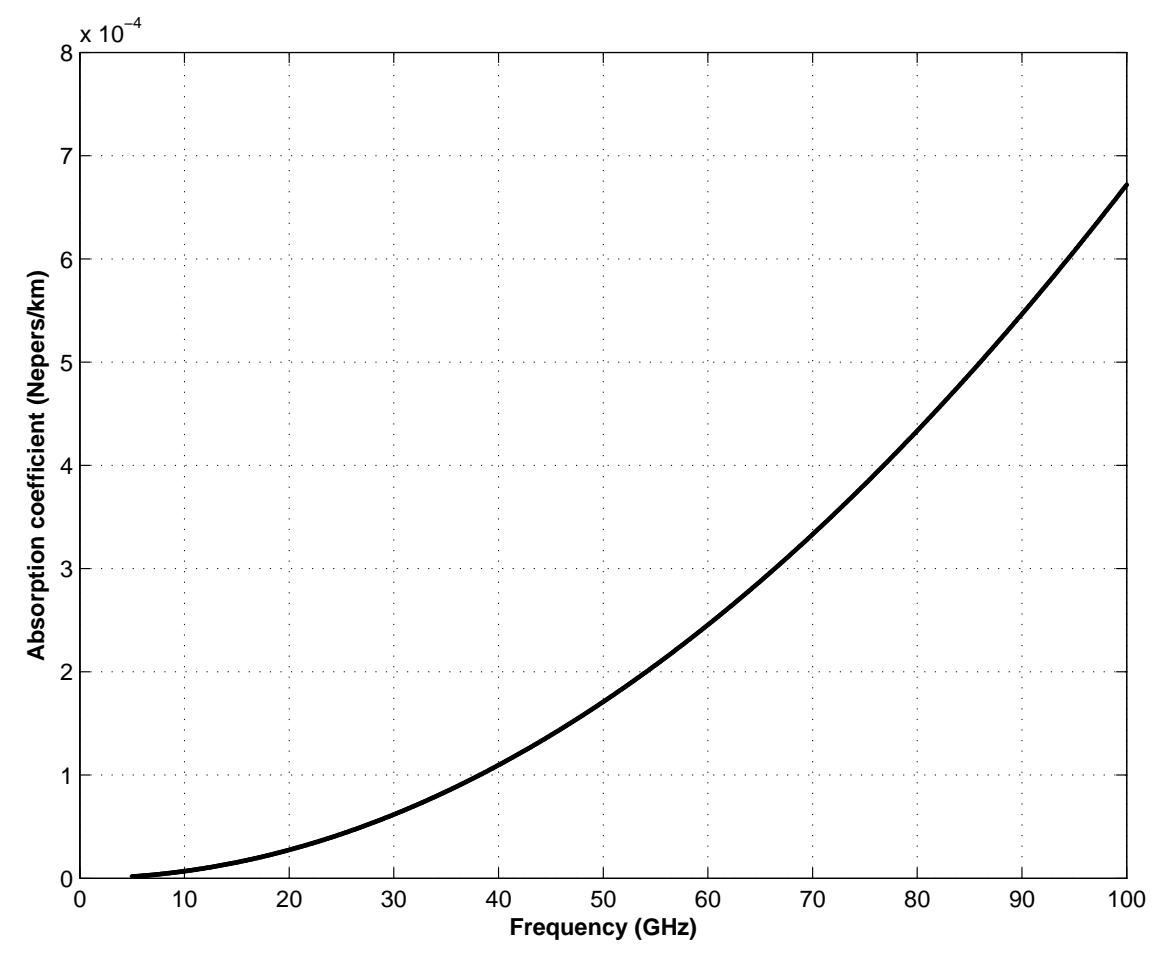

Figure 2.10: Frequency spectrum of N2 absorption.

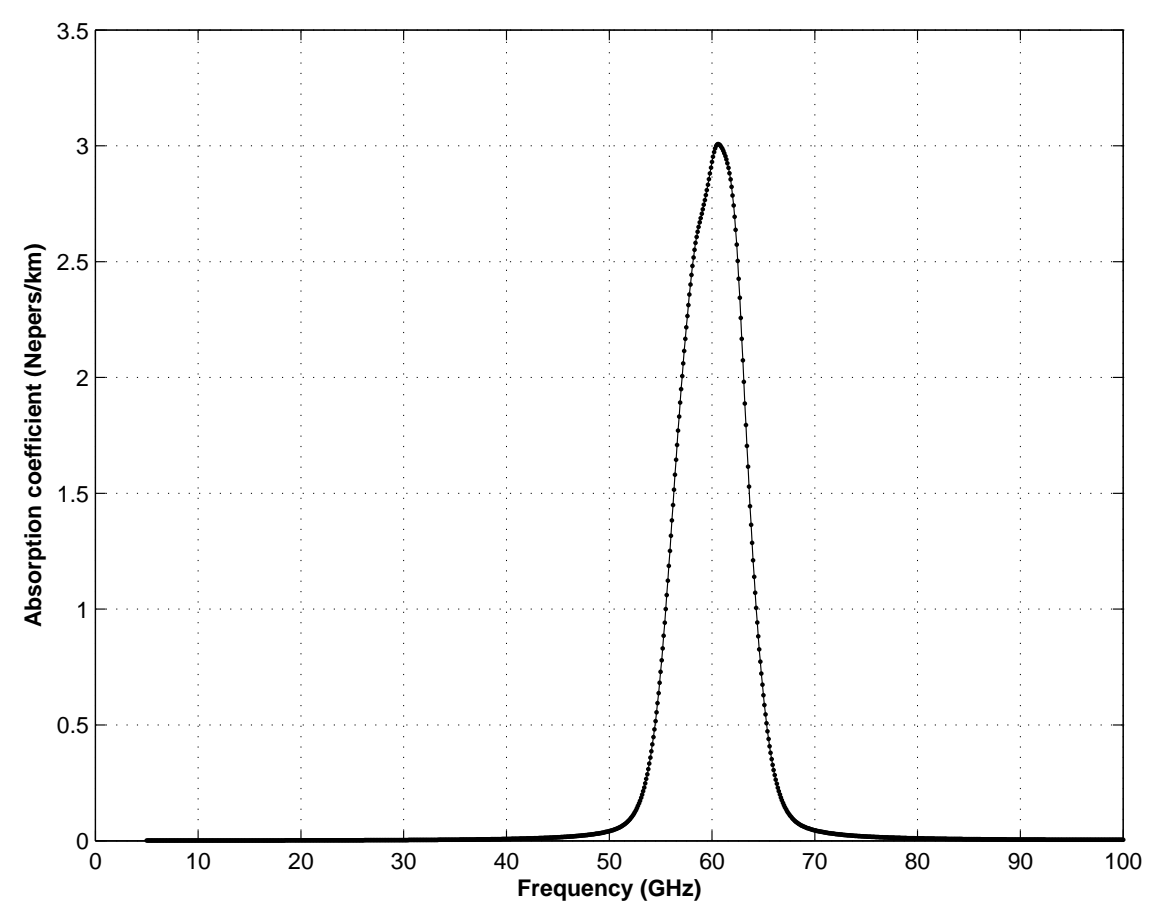

Figure 2.11: Frequency spectrum of O2 absorption at sea level pressure. 
The environmental inputs to the RTM are obtained from the NOAA Global Data Assimilation System (GDAS) archive, which provides model outputs at 0000, 0600, 1200 and 1800 GMT and on a $100 \mathrm{~km}$ grid. These data include the atmospheric profiles of pressure, temperature, and water vapor at 21 levels as well as columnar cloud liquid water, sea surface temperature, and ocean wind speed at $10 \mathrm{~m}$ height. The atmosphere is divided into 100 layers of $200 \mathrm{~m}$ thickness each. Therefore, the atmosphere is modeled up to a height of $20 \mathrm{~km}$, which extends beyond the height of the tropopause [30]. The air in the atmosphere above the tropopause is extremely rare and does not significantly affect the apparent radiometer Tb. Thus, the RTM adequately models the entire extent of the atmospheric contribution to the radiometer $\mathrm{Tb}$. The atmospheric profiles from GDAS are interpolated to the heights of the 100 layers in the RTM, using a piece-wise linear distribution for temperature and piece-wise exponential distributions for pressure and water vapor. The lapse rates of the temperature, pressure and water vapor have significant differences between the upper and lower layers for some cases. Thus, generating a single fit for the entire vertical profile would have resulted in large re-sampling errors for these cases. Therefore, piecewise interpolations were used to adequately represent the non-uniform variation of environmental parameters in the different layers. A uniform distribution is used for cloud liquid water, and the heights of the cloud top and bottom are derived from ocean climatology based on the ENVIMOD model developed by Wisler and Hollinger [31]. These environmental parameters are matched-up with the mean TMI Tb observations for each 1 degree box and are used to calculate the predicted $\mathrm{Tb}$ for that box. 


\subsection{Radiometric Inter-comparison of TMI with Windsat and}

\section{SSMI}

The radiometric inter-calibration is performed using more than 100,000 spatial/temporal collocations between TMI and WindSat and more than 300,000 collocations between TMI and SSMI F-13 and F-14.

The RTM is used to predict differences of pair-wise radiometer channels over a wide variety of environmental conditions. To normalize the $\mathrm{Tb}$ measurements before pair-wise comparisons, the difference between the predicted values of two radiometer channels is calculated, by running the RTM for all frequencies in the bandwidth of each channel with small increments. The integral of the $\mathrm{Tb}$ over the radiometer channel bandwidth is approximated using Simpson's rule and the average was calculated. Afterwards the difference between channels is determined, for each input set of environmental conditions, as follows :

$$
\begin{aligned}
\Delta & =T b_{-} T M I p r e d-T b_{-} S S_{\text {pred }} \\
T b \_S S_{n o r m} & =T b_{-} S S_{o b s}+\Delta \\
T b \_d i f f & =T b_{-} T M I_{o b s}-T b_{-} S S_{\text {norm }}
\end{aligned}
$$

where

$\Delta$ is the expected Tb difference between TMI and the sun-synchronous satellite; 
$T b_{-} S S_{\text {norm }}$ is the Sun Synchronous (i.e. WindSat or SSMI) Tb normalized to the TMI incidence angle and center frequency;

$T b \_S S_{o b s}$ is the Sun Synchronous observed Tb;

$T b \_T M I_{\text {pred }}$ is the RTM Tb prediction at the TMI frequency and incidence angle; $T b_{-} S S_{\text {pred }}$ is the RTM Tb prediction at the Sun Synchronous radiometer's frequency and incidence angle;

Tb_diff is the normalized Tb difference between TMI and WindSat/SSMI ; and Tb_TMI $I_{o b s}$ is the TMI observed Tb.

This process is illustrated graphically in Fig. 2.12, where panel-(a) is a scatter diagram of binned averages of "raw" WindSat 10V and collocated TMI measurements; and the dashed line depicts the 45 deg line where TMI and WindSat measurements are equal. The WindSat measurements are lower than TMI measurements by $\sim 5-6 \mathrm{~K}$; however, the expected difference between TMI and WindSat is about $6.5 \mathrm{~K}$ (see panel-b). This predicted $\mathrm{Tb}$ difference is primarily due to the incidence angle difference of $\sim 3^{\circ}$ between the two channels and a smaller expected $\mathrm{Tb}$ difference $(\sim 0.3 \mathrm{~K})$ due to the $0.05 \mathrm{GHz}$ frequency difference. Also, this expected difference varies with the environmental parameters associated with the match-up collocations, which explains the change with TMI Tb shown in panel-(b).

Thus, taking the difference of $\mathrm{Tb}$ differences (observed and expected) results in the unexplained bias for the $10 \mathrm{~V}$ channel, which is less than $1.5 \mathrm{~K}$. After normalizing the WindSat $10 \mathrm{~V} \mathrm{~Tb}$ according to (2.4), the results are presented in panel-(c) and -(d), which exhibit both offset and slope differences between these radiometers. 


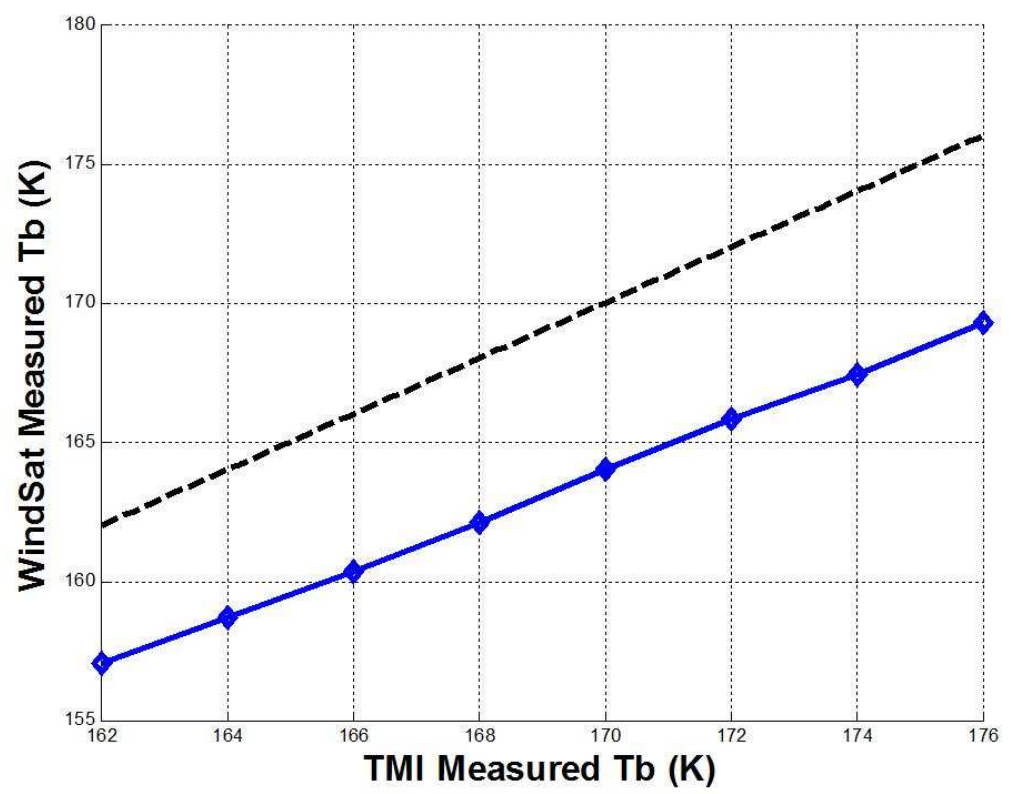

(a) Raw Tb observations.

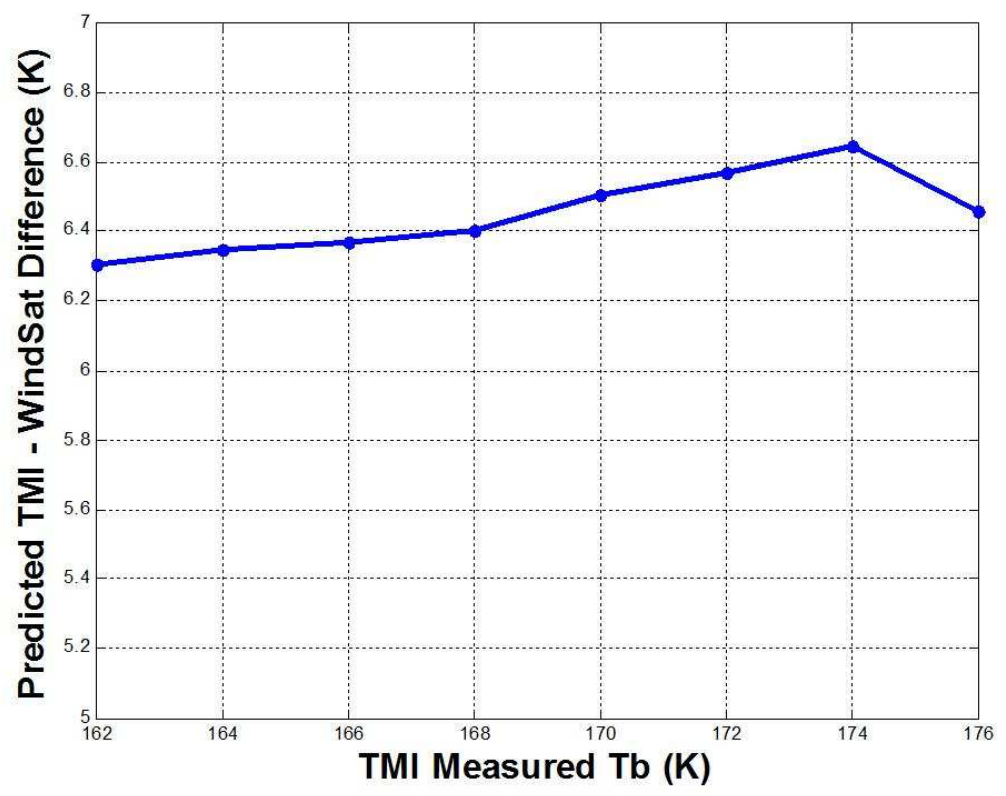

(b) Expected Tb difference predicted by RTM.

Figure 2.12: Normalized WindSat 10V Tb observations to corresponding TMI channel frequency and incidence angle. 


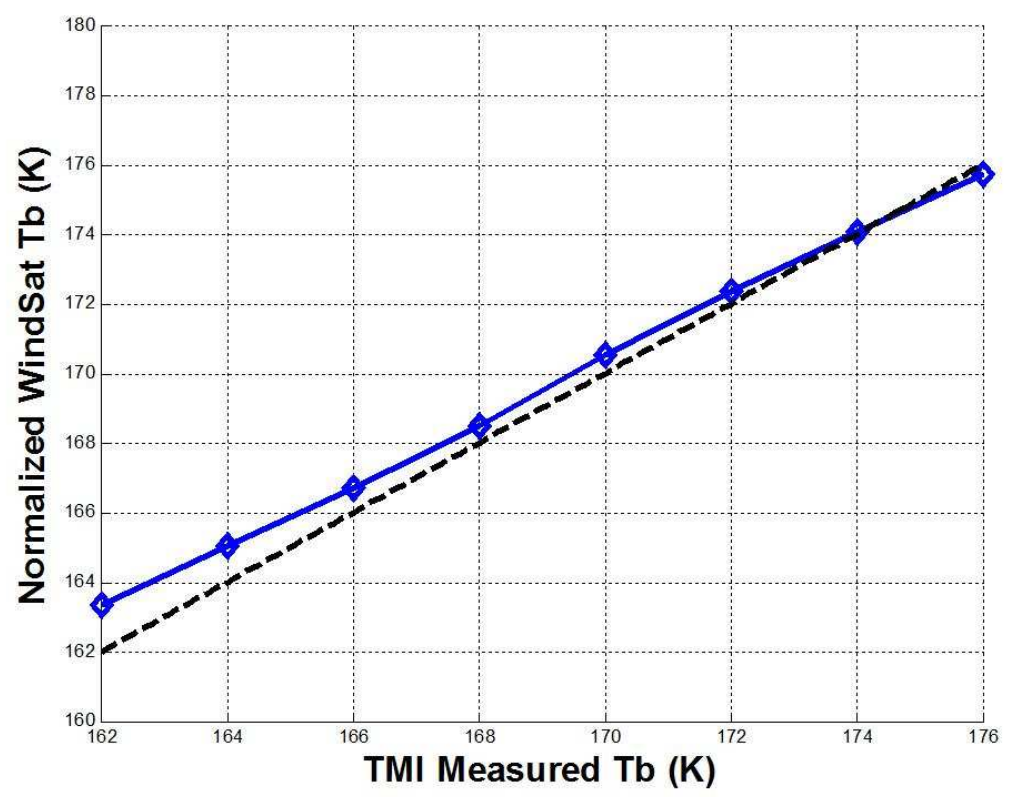

(c) Normalized WindSat and TMI Tb comparisons.

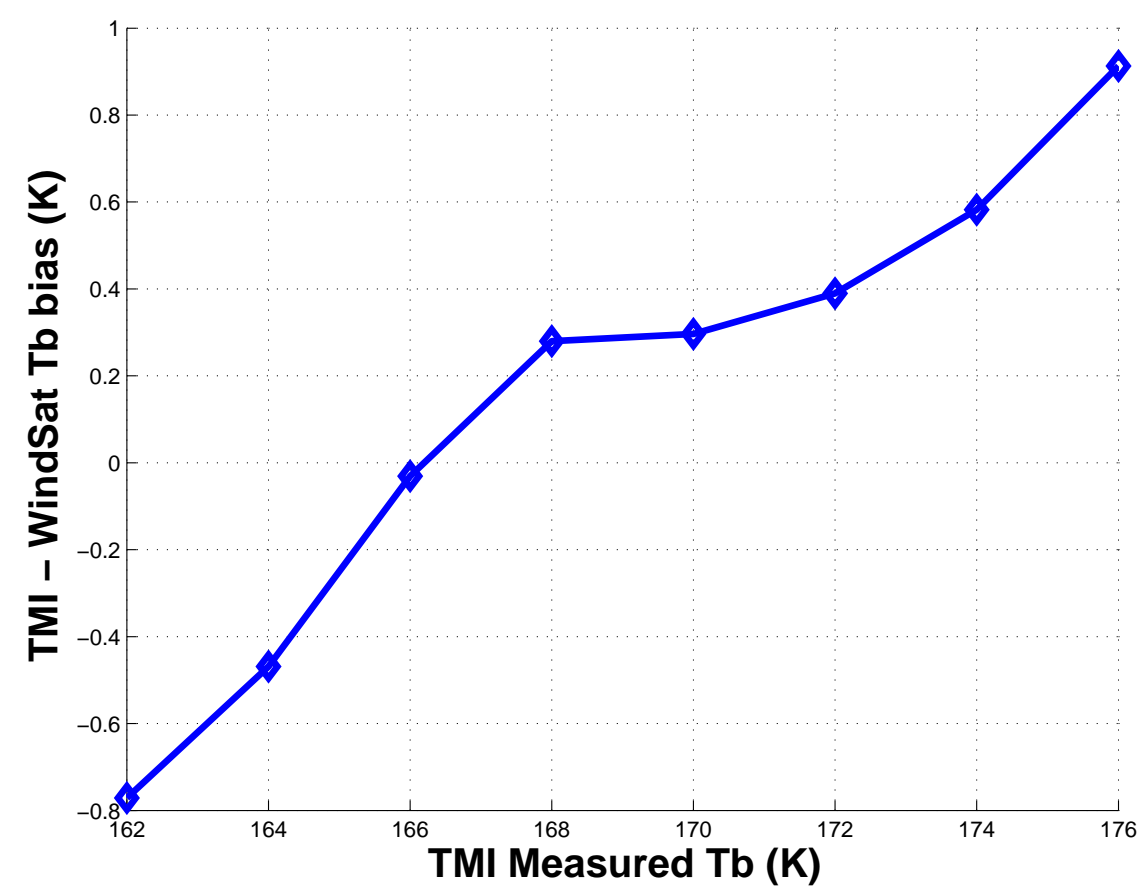

(d) TMI Tb biases relative to WindSat.

Figure 2.12: Normalized WindSat 10V Tb observations to corresponding TMI channel frequency and incidence angle. 
The corresponding 37V channel comparisons shown in Fig. 2.13 are similar to the $10 \mathrm{~V}$ results. In panel-(a), the mean WindSat 37V Tb's are lower than collocated TMI measurements by $\sim 3-5 \mathrm{~K}$; however in this case, the predicted (expected) difference between TMI and WindSat (panel-(b)) is only $<0.5 \mathrm{~K}$ because the TMI and WindSat channels have nearly identical frequencies and incidence angles. Thus, at $37 \mathrm{GHz}$, the majority of the difference in the TMI and WindSat observations (see panels-(c) and -(d)) is caused by unexplained radiometric calibration bias (and a similar small slope difference). Of course these biases and slopes are the required brightness temperature adjustments necessary to bring these two radiometers into agreement; however, this does NOT imply which (if either) is correct. Plots of the measured, predicted and normalized differences between TMI and WindSat for all the TMI channels are found in Appendix B.

The relative biases between TMI and WindSat were calculated for all the match-up samples in the ICWG time period. The histogram of the relative biases of the $10 \mathrm{~V}$ channel, displayed in Fig. 2.14, shows that the relative biases have a bi-modal distribution. The peaks of the histogram are at approximately $-0.5 \mathrm{~K}$ and $1 \mathrm{~K}$. Based on previous inter-calibration efforts $[10,32]$, a systematic calibration error source is expected to result in small random deviations of the error around a constant mean value. For a large number of statistically independent samples, this is expected to result in a Gaussian distribution around the mean value. Thus, the relative biases in the $10 \mathrm{~V}$ channel can be thought of as being caused by two distinct random error sources with different means. 


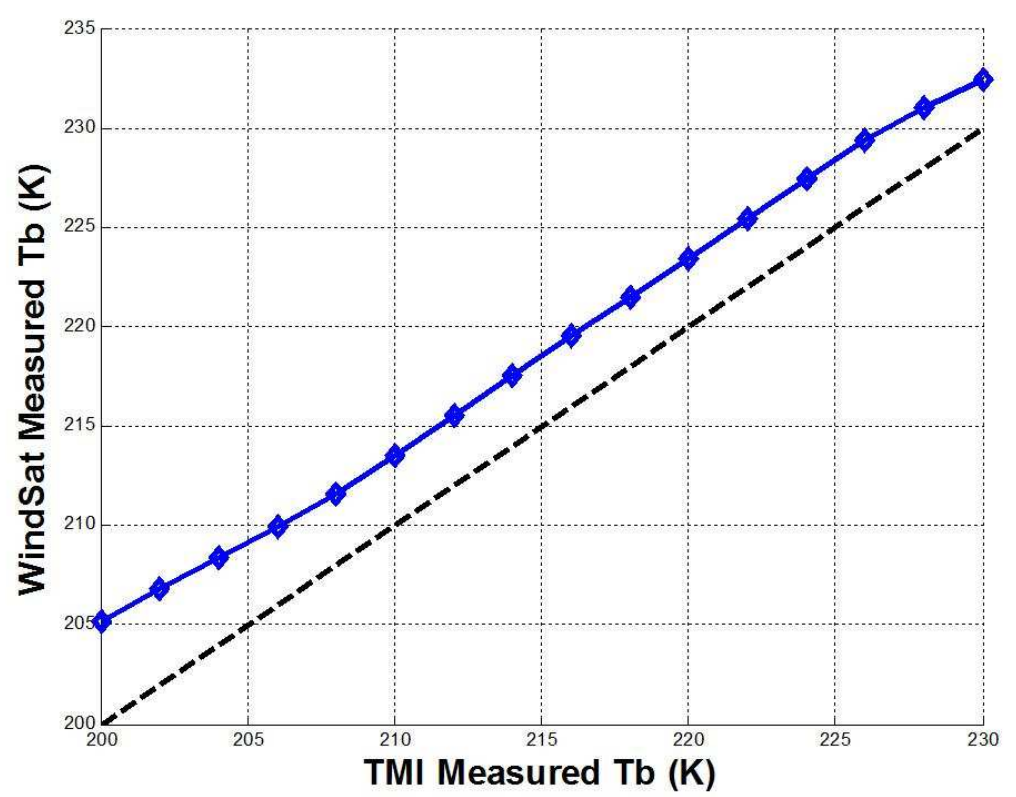

(a) Raw Tb observations.

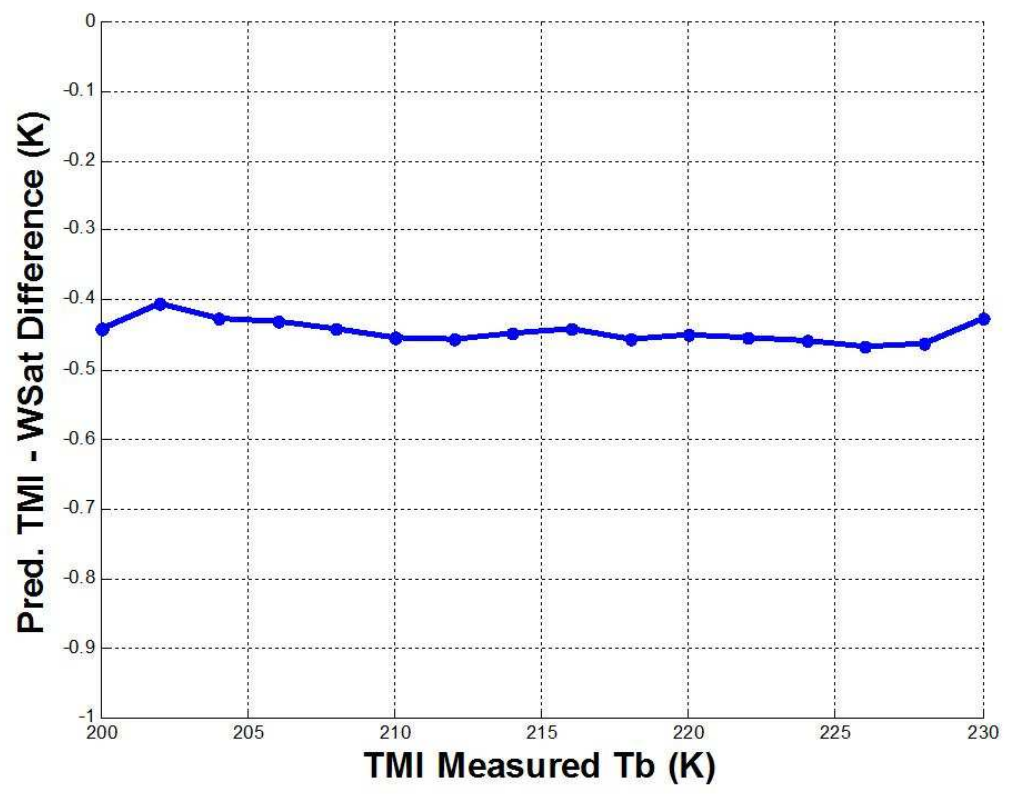

(b) Expected Tb difference predicted by RTM.

Figure 2.13: Normalized WindSat 37V Tb observations to corresponding TMI channel frequency and incidence angle. 


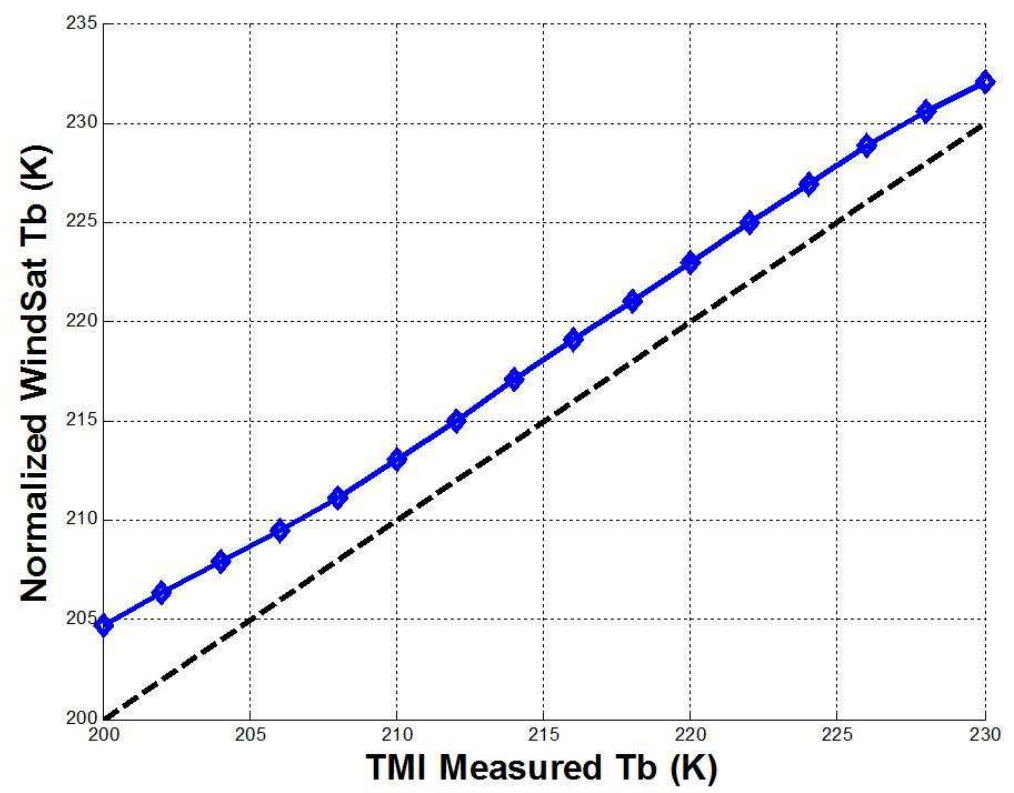

(c) Normalized WindSat and TMI Tb comparisons.

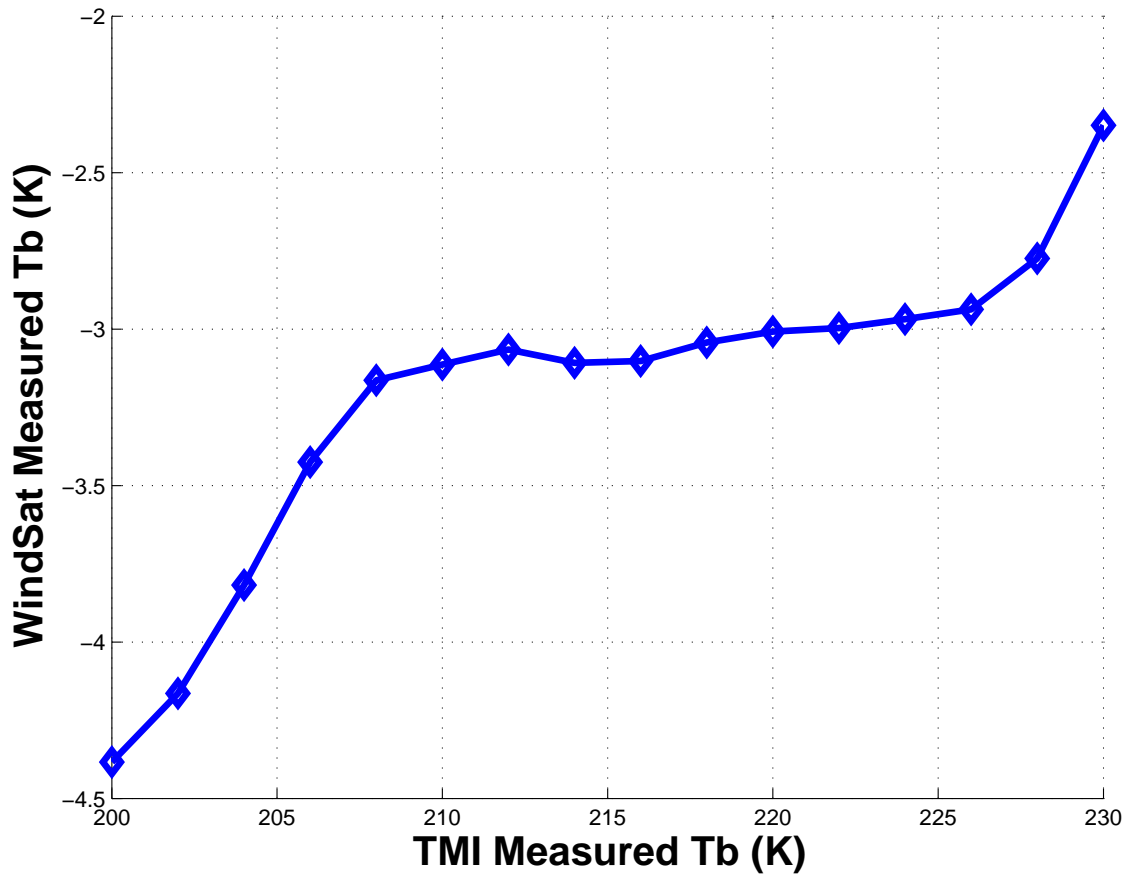

(d) TMI Tb biases relative to WindSat.

Figure 2.13: Normalized WindSat 37V Tb observations to corresponding TMI channel frequency and incidence angle. 


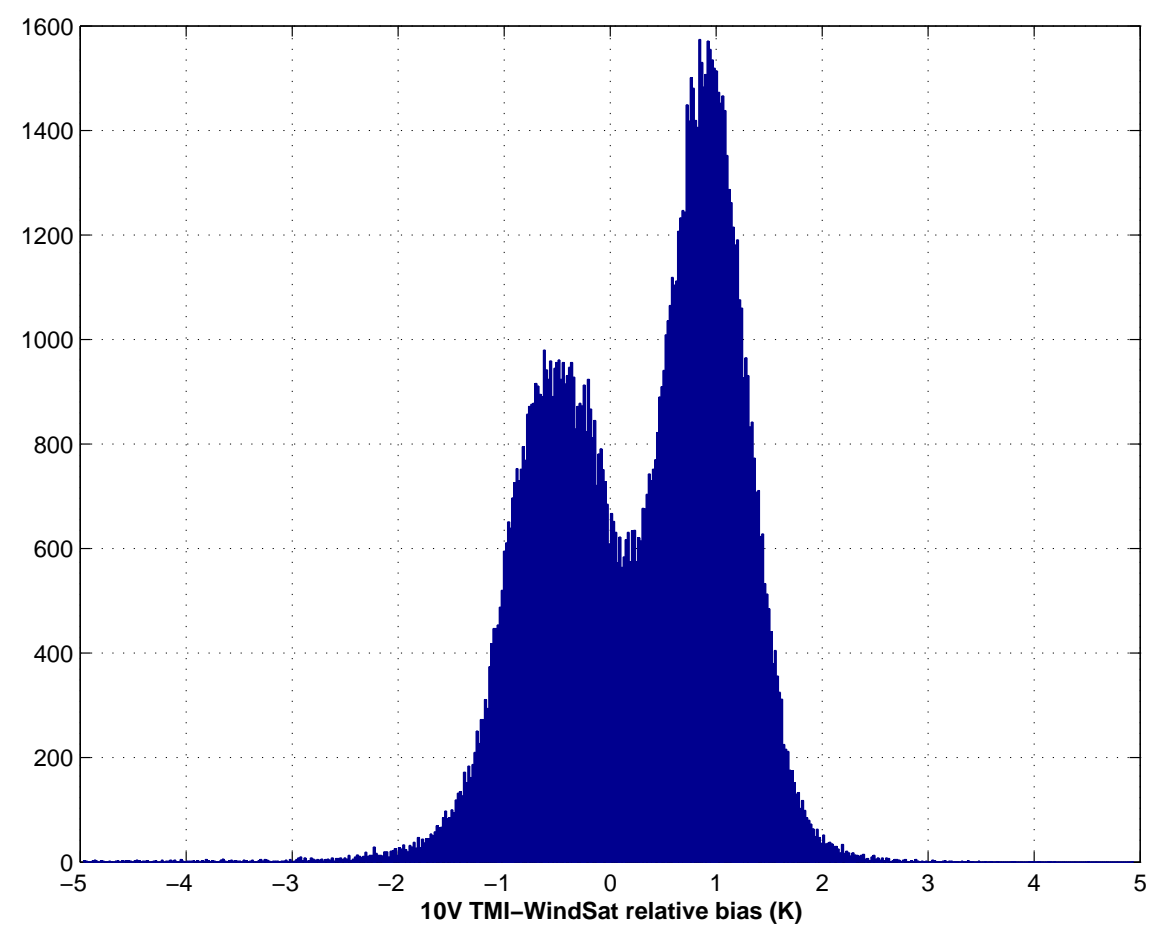

Figure 2.14: TMI and WindSat 10V unexplained Tb bias distribution.

In an attempt to understand the source of the two distinct biases, the comparison data set was sorted in different ways; and an example is presented in Figs. 2.15 and 2.16, where the $\mathrm{V}$-pol and $\mathrm{H}$-pol relative biases were plotted against the corresponding binned values of SST. We expect that radiometric biases should not be a function of environmental parameters, yet the $\mathrm{Tb}$ biases of the $10 \mathrm{~V}, 10 \mathrm{H}, 37 \mathrm{~V}$ and $37 \mathrm{H}$ channels exhibited patterns that were highly correlated. In each, there appeared to be a low bias plateau in the range of SST $<290 \mathrm{~K}$ followed by a rapid step in Tb bias of $\sim 0.3-0.4 \mathrm{~K}$ to a plateau for SST $>295 \mathrm{~K}$. The patterns for the 19 and $21 \mathrm{GHz}$ channels were not so clear, but there was generally an increasing bias with higher SST. So initially this investigation focused on the 10 and $37 \mathrm{GHz}$ channels, and V-pol was selected because of its reduced component of reflected downwelling atmospheric brightness. 




(a) $10 \mathrm{~V}$

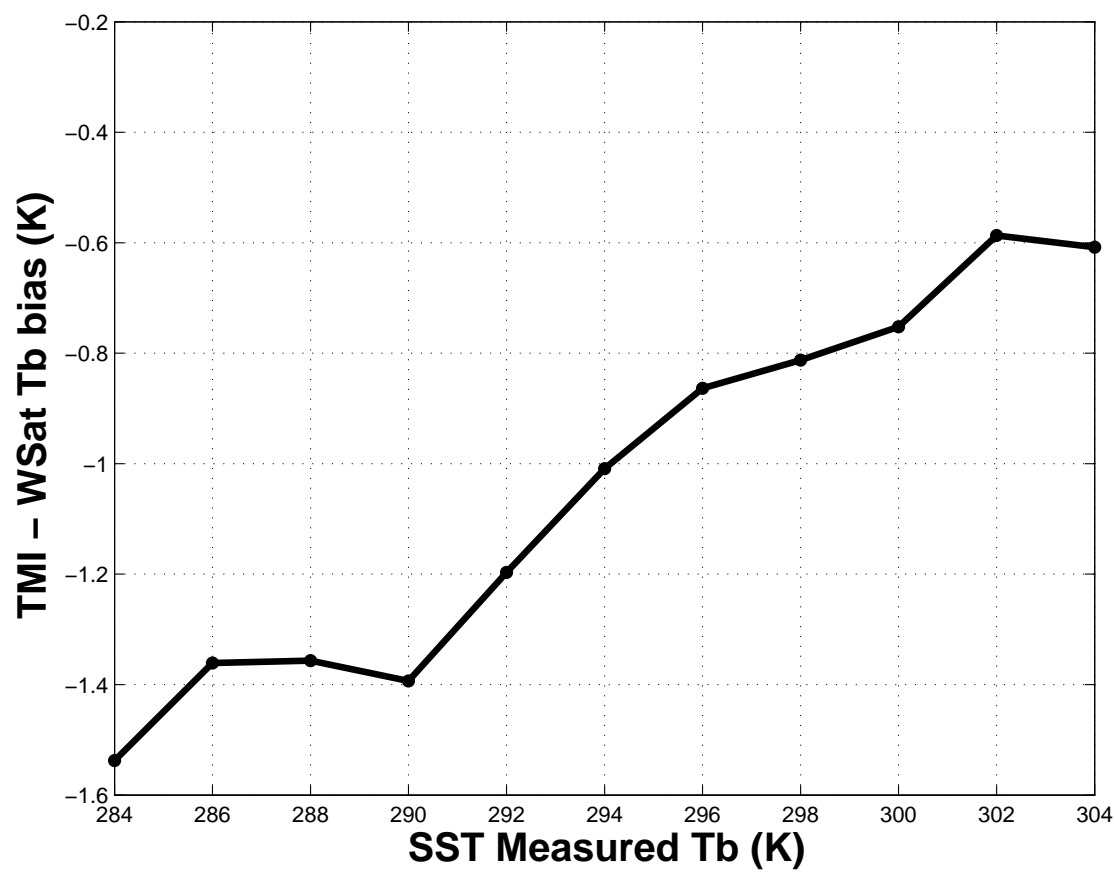

(b) $19 \mathrm{~V}$

Figure 2.15: TMI-WindSat bias SST dependence for V-pol channels. 


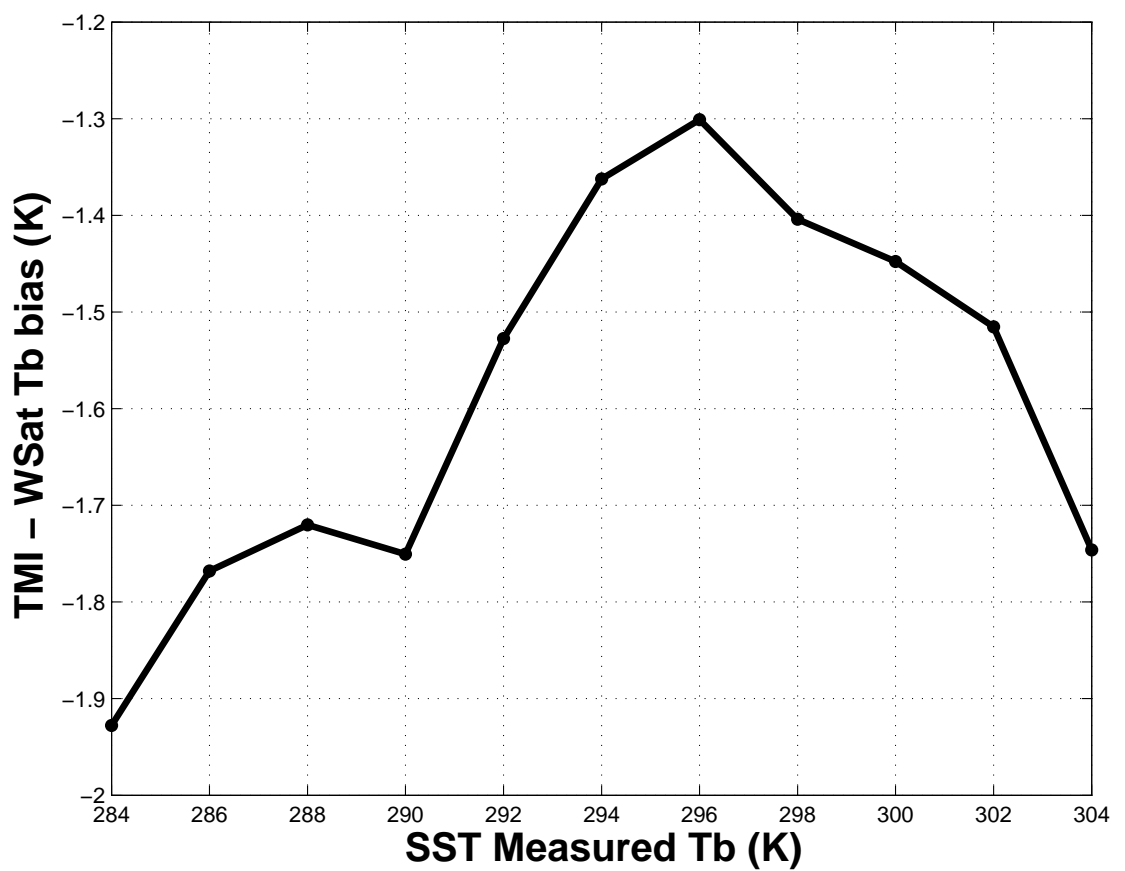

(c) $21 \mathrm{~V}$

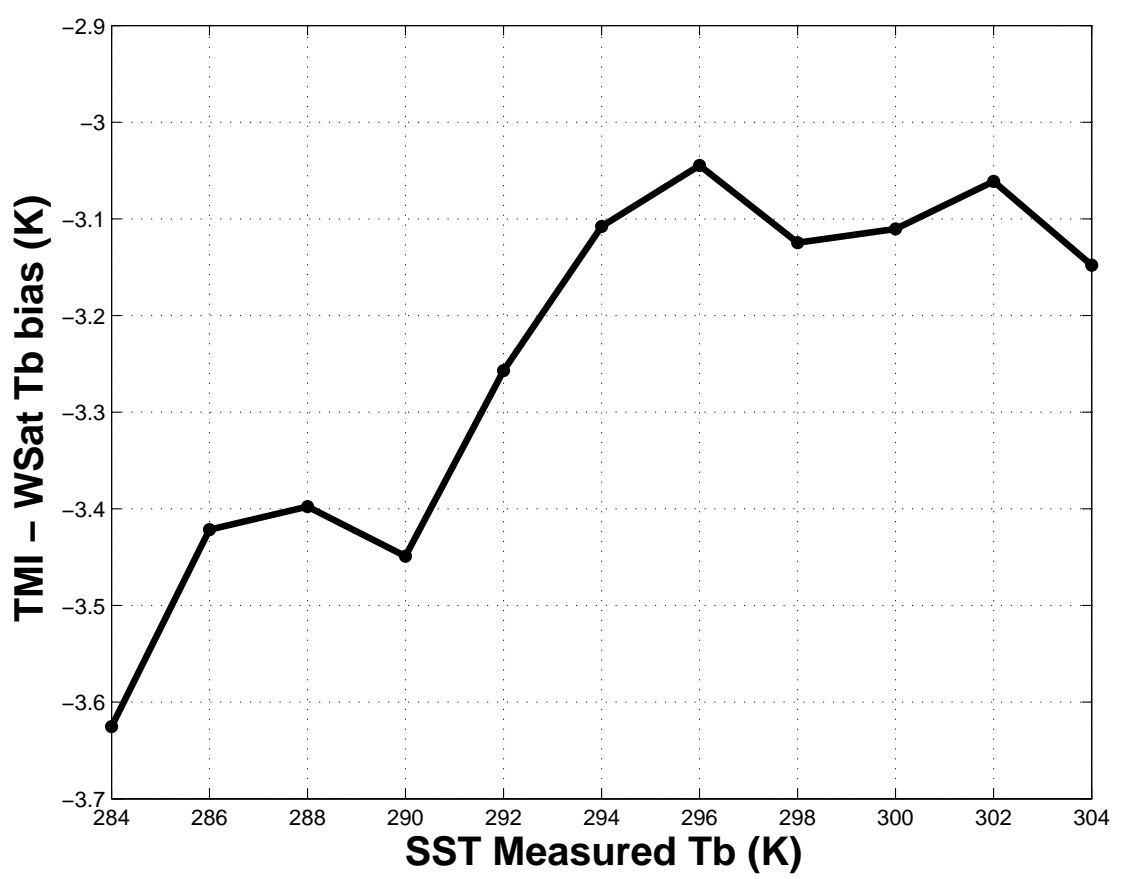

(d) $37 \mathrm{~V}$

Figure 2.15: TMI-WindSat bias SST dependence for V-pol channels. 


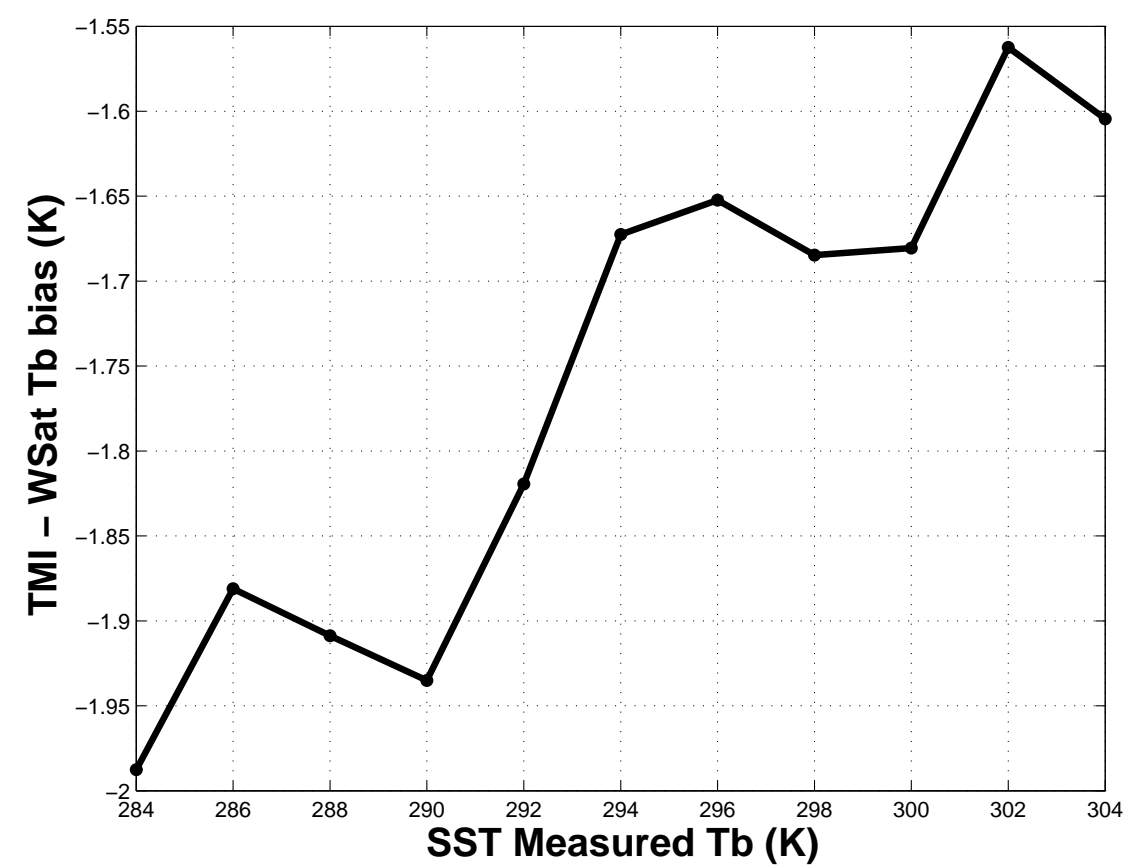

(a) $10 \mathrm{H}$

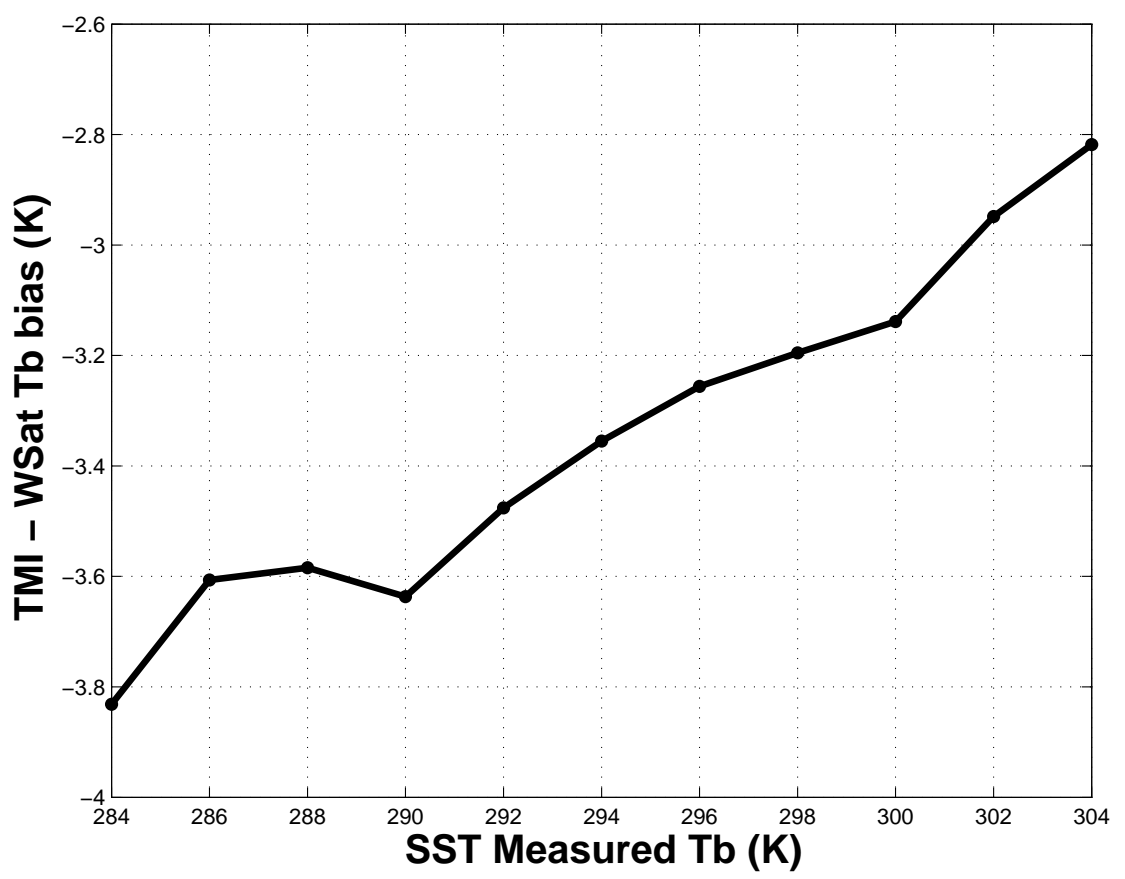

(b) $19 \mathrm{H}$

Figure 2.16: TMI-WindSat bias SST dependence for H-pol channels. 


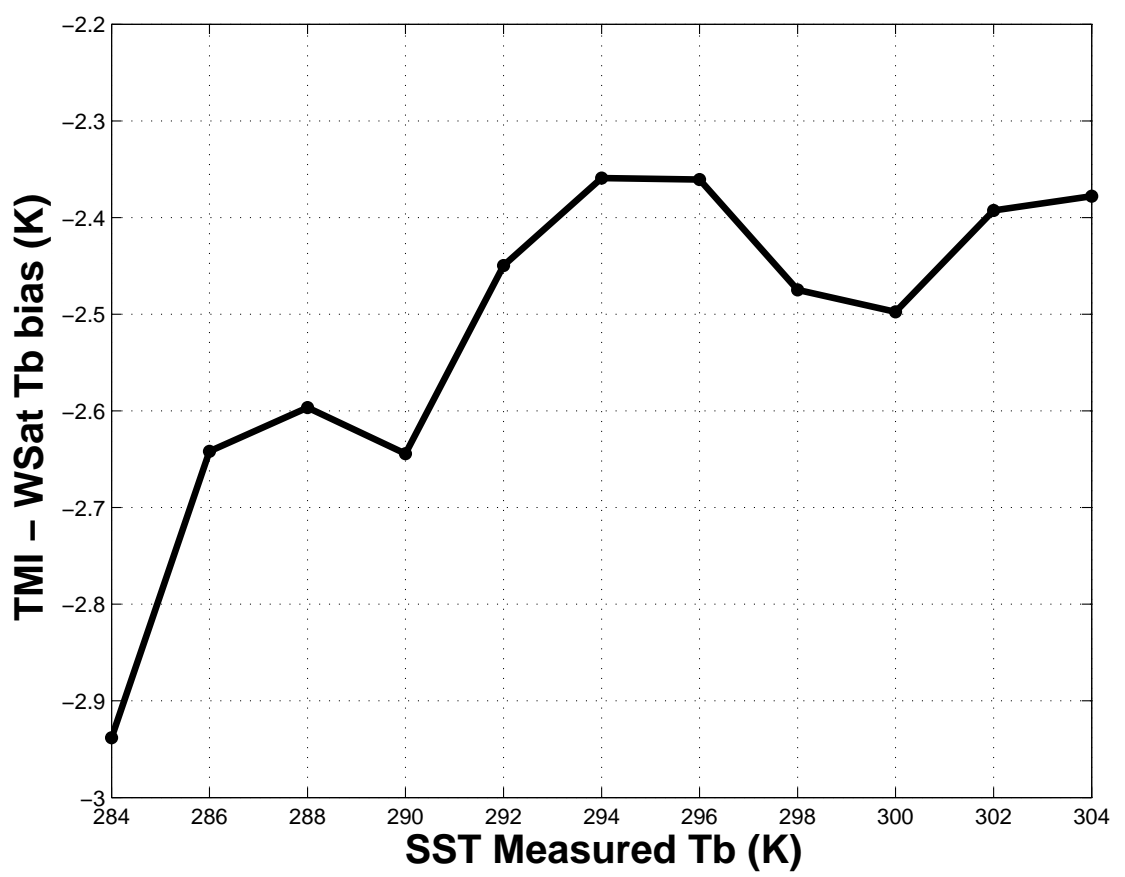

(c) $37 \mathrm{H}$

Figure 2.16: TMI-WindSat bias SST dependence for H-pol channels.

We asked ourself the question "How does the theoretical ocean brightness temperature respond for these TMI channels?" To answer this, we ran the RTM parametrically by holding all environmental parameters fixed except for the one of interest. An example of the theoretical ocean surface component of brightness temperature, which makes up $>90 \%$ of the total apparent $\mathrm{Tb}$, is shown in Fig 2.17. It is noted that the $10 \mathrm{~V}$ channel has a $\mathrm{Tb}$ response that is linearly dependent on SST; while the $37 \mathrm{~V}$ channel is nearly constant and does not change with varying SST. Given these very different theoretical responses, it is highly unlikely that the apparent "step-function" change in the TMI/WindSat bias is related to the SST. Furthermore, the bias should not correlate with any environmental parameter; so plotting the biases versus SST (or any other parameter) should result is a scatter diagram that is a circular random pattern. Clearly this is not the present situation, thus this hypothesis fails 
in this case. So, we recognize that the biases should be independent of SST; but the results are NOT random; therefore, there is some other cause for the step-function radiometric bias that happened to be correlated with the SST.

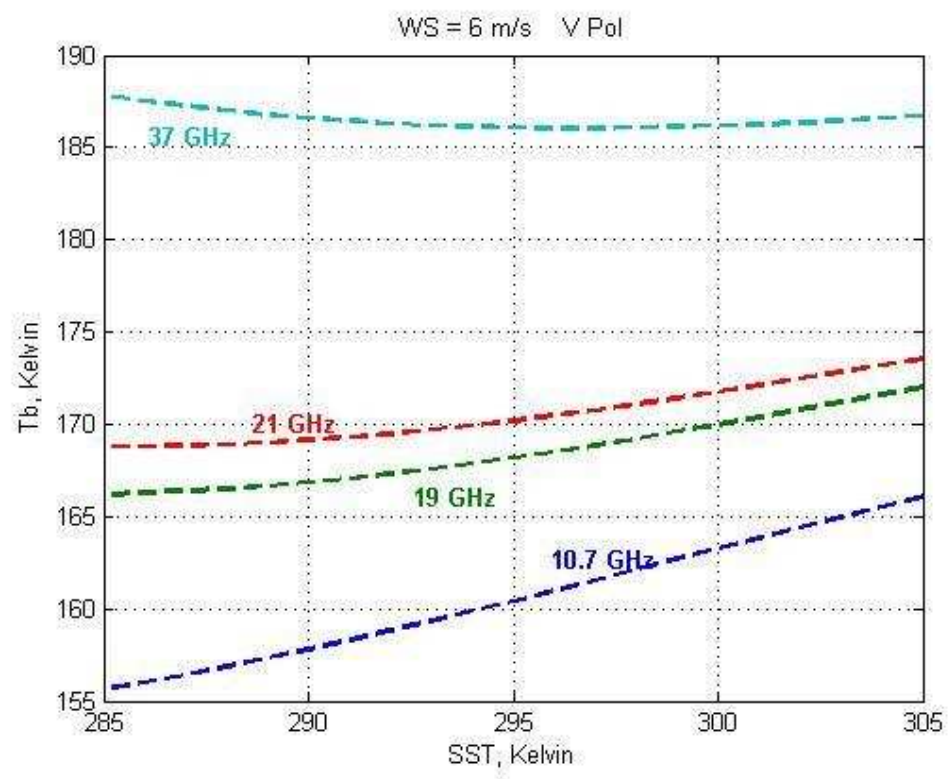

(a) Vertical polarization

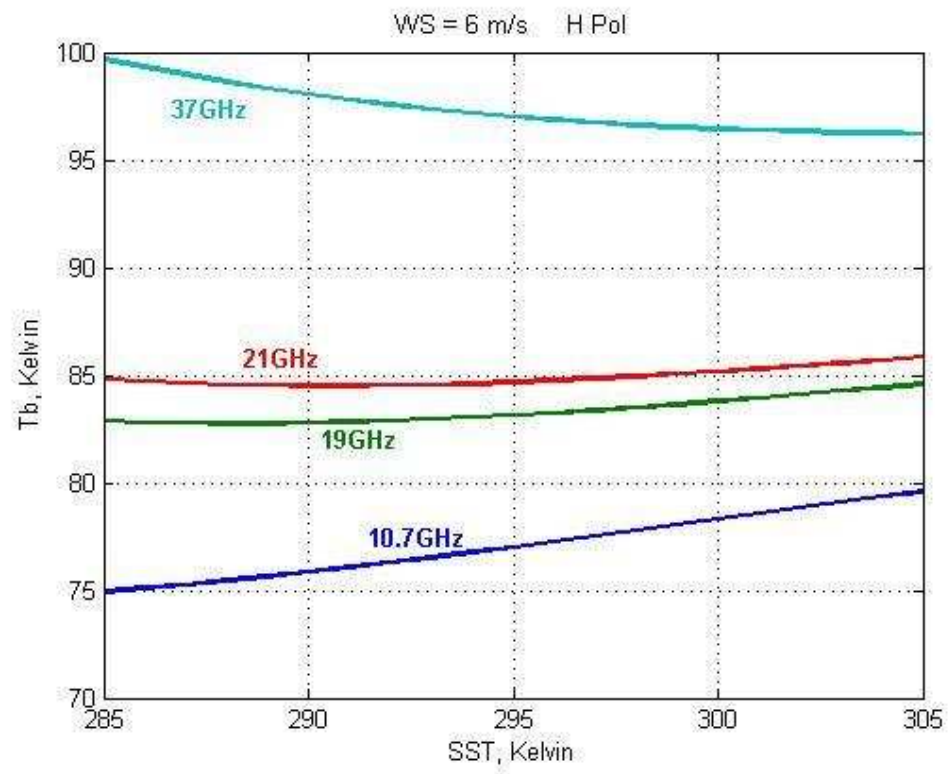

(b) Horizontal polarization

Figure 2.17: Theoretical ocean surface brightness temperature for an incidence angle of 53 deg and an ocean wind speed of $6 \mathrm{~m} / \mathrm{s}$ and a salinity of $32 \mathrm{ppt}$. 
To further investigate the cause for this anomaly, we next examined the dependence of the theoretical Tb's at the $10.7 \mathrm{GHz}$ frequency. We picked $10.7 \mathrm{GHz} \mathrm{V}$-pol, because we have the highest confidence in our ability to accurately model this channel. The spatial/temporal variations of $\mathrm{Tb}$ are gradual because of the uniformity of ocean SST and the effects of the transient atmospheric component of brightness temperature are small $(<10 \mathrm{~K})$. Since $10 \mathrm{~V} \mathrm{~Tb}$ is primarily sensitive to the ocean surface emission and since the emissivity of a calm ocean surface depends on the dielectric constant of sea water and Fresnel reflection coefficients, both of which are well known, we expect the RTM calculations to be robust.

The RTM calculations of the expected differences and the observed differences between the TMI and WindSat raw (not normalized) Tb's are plotted against the corresponding binned average SST's and are presented in Fig. 2.18. From this figure, it is evident that the step in the relative biases is caused by the measured Tb's, which eliminates the possibility that this observation is somehow related to modeling errors in the RTM. So, it was concluded that the unexpected step response was caused by an anomaly in the measurements that happened to be correlated with SST. 


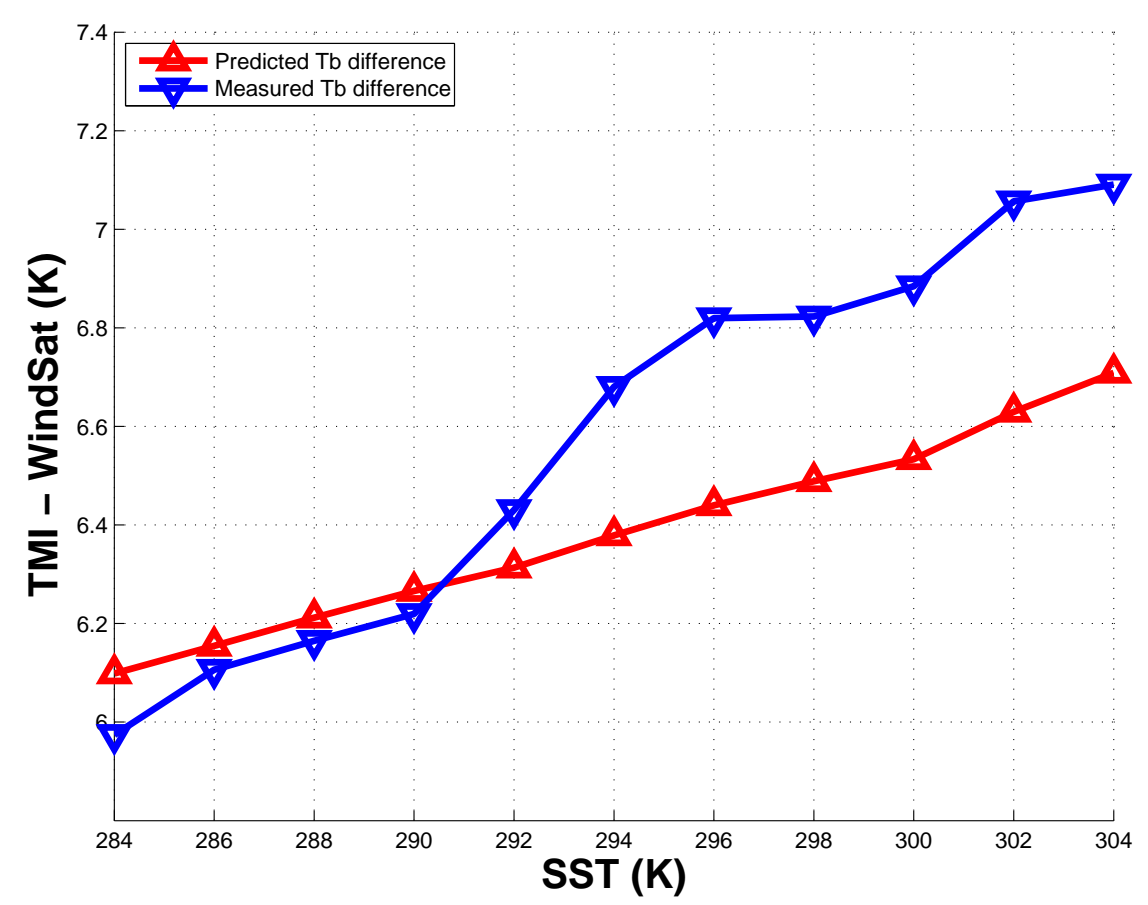

Figure 2.18: TMI-WindSat predicted and measured Tb differences.

Since SST is a maximum at the equator and cools toward the poles, there is a strong correlation with latitude. Also there is a weak diurnal (time of day) SST signature; and the atmospheric water vapor content is highly correlated. Thus, the dependence of the estimated bias on these three parameters was investigated.

First, the effect of incidence angle changes with latitude was examined. Figure 2.19 shows that there is a systematic (but insignificant $\sim 0.05$ deg peak) in the incidence angle difference between TMI and the WindSat $10 \mathrm{GHz}$ channel over $\pm 30 \mathrm{deg}$ of latitude, At the extremes of the TMI swath (beyond $|30|$ deg), the incidence angle difference was observed to change rapidly ; but even here the magnitude $(\sim 0.05 \mathrm{deg})$ results in an insignificant change in the $\mathrm{Tb}$ bias. These abrupt changes in the incidence angle can be explained by the azimuth dependence of the TMI incidence angle, which is shown in Fig. 2.20. The 
incidence angles on the right side of the scan are higher for the period when TRMM flies in the $+\mathrm{X}$ direction (zero deg yaw), and the incidence angles are higher on the left side of the scan when TRMM flies in the -X direction (180 deg yaw). At latitudes $>30$ degrees, the observations are skewed towards the azimuth scan pixels to the left side for the forward yaw orientation, and the pixels to the right side for the backward yaw orientation. The incidence angle difference is therefore lower at the high northern latitudes. Similarly, at latitudes $<-30$ degrees, the observations are skewed towards the left side for the forward yaw orientation, and to the right side for the backward yaw orientation. Thus, the average incidence angle difference is higher at the extreme southern latitudes. On the other hand, at latitudes closer to the equator all azimuths are included, and the incidence angle only changes gradually with latitude. Furthermore, given typical partial derivatives of Tb with respect to incidence angle of $1-2 \mathrm{~K} / \mathrm{deg}$, the change in incidence angle is at least one-order of magnitude too small to account for the observed bias step $(0.3-0.4 \mathrm{~K})$. Finally, separating the TMI-WindSat matchups into two sets at high and low latitudes and then calculating the Tb biases resulted in negligible changes in the "step SST response", as shown in Fig. 2.21. This disproves the hypothesis that latitude dependent incidence angle variations caused this apparent $\mathrm{Tb}$ anomaly. 
Delta EIA (TMI - WS)

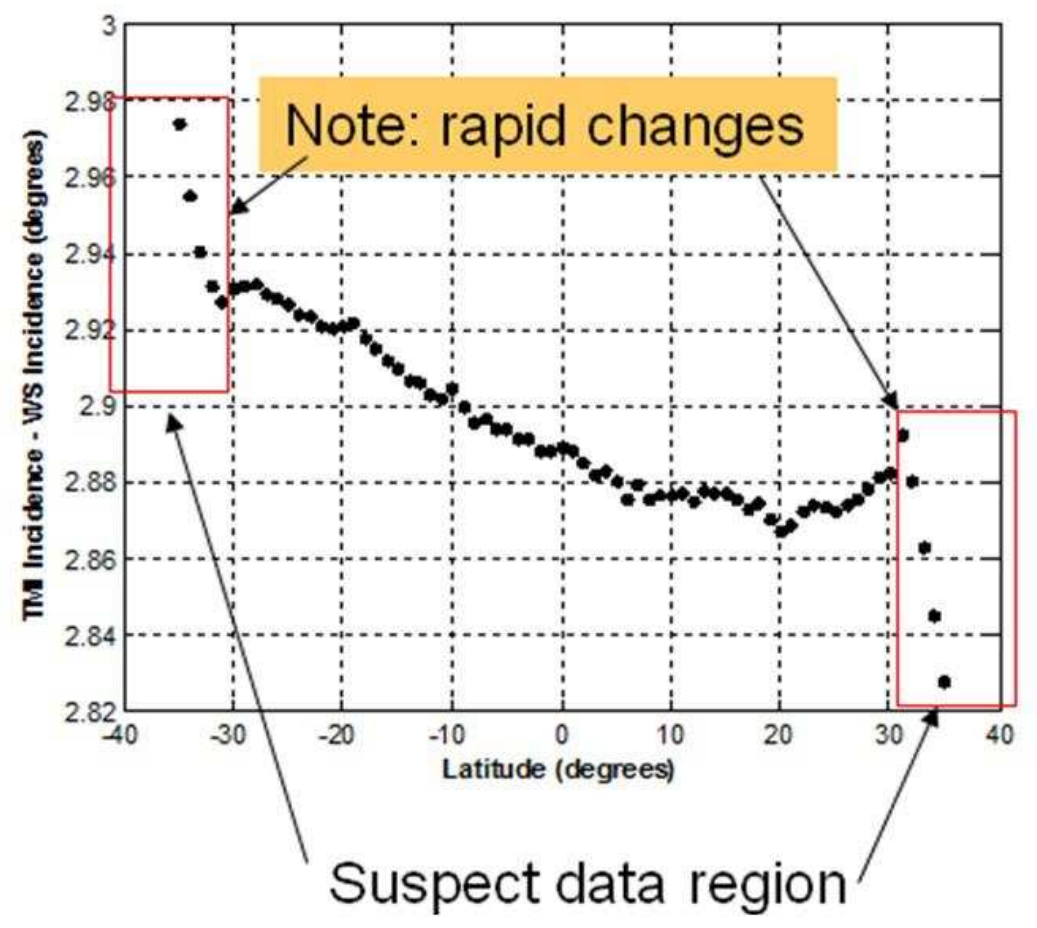

Figure 2.19: TMI/WindSat Incidence angle difference variation with latitude. 


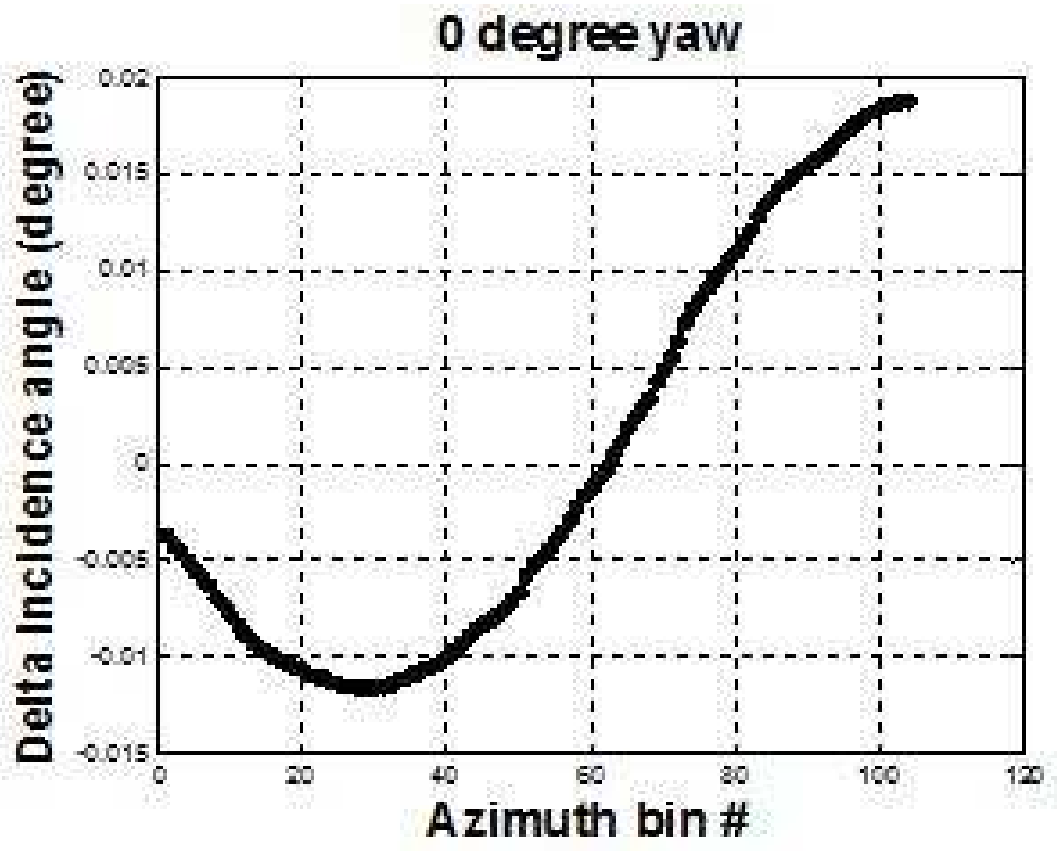

(a) 0 degree yaw.

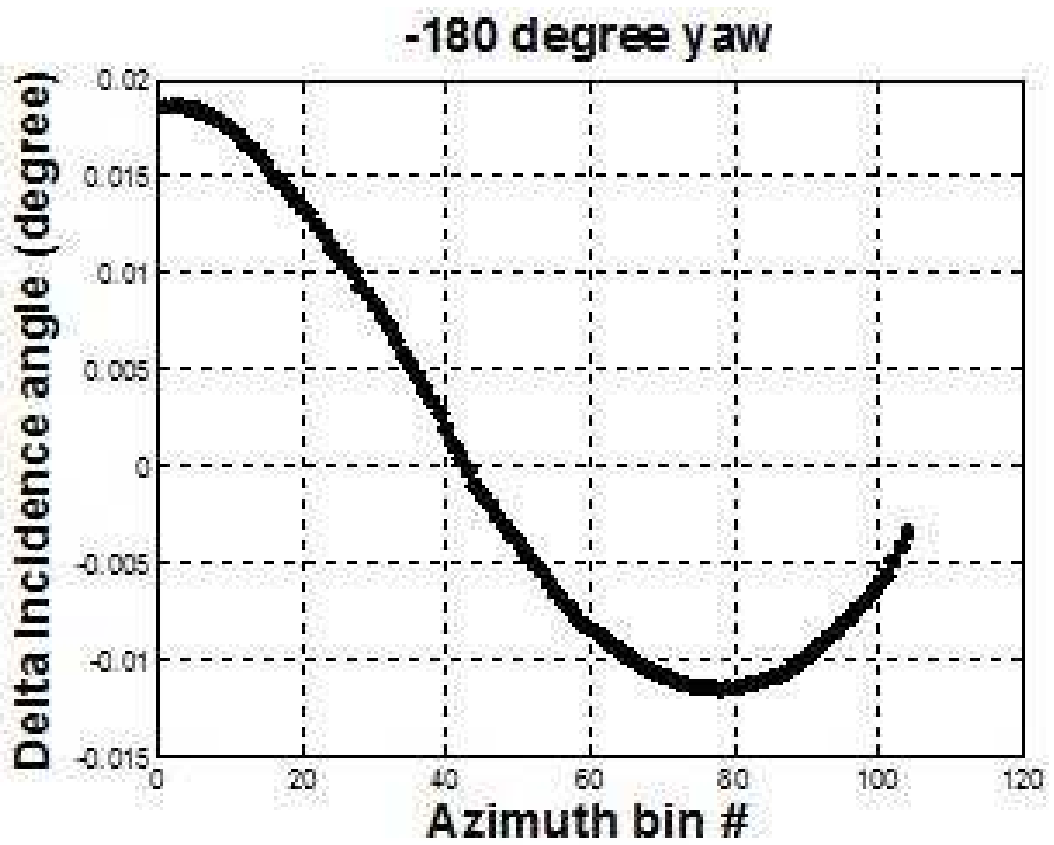

(b) 180 degree yaw.

Figure 2.20: TMI incidence angle variation with scan azimuth position. 


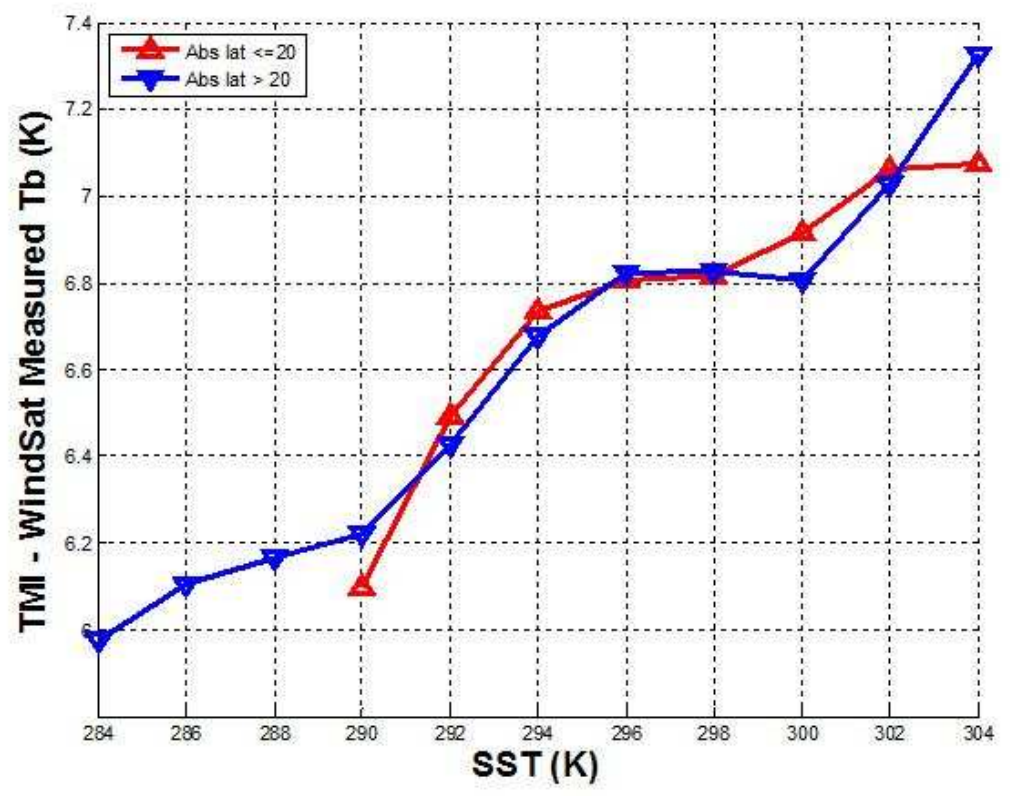

Figure 2.21: Latitude dependence of TMI-WindSat 10V measured Tb differences.

Next, we examined the influence of water vapor in the RTM calculations as being the possible cause for this Tb bias anomaly. The dependence of the TMI - WindSat differences for the $10 \mathrm{~V}$ and $21 \mathrm{~V}$ channels on columnar water vapor is shown in Fig. 2.22. The most notable feature is a steep dip in the $21 \mathrm{~V}$ bias value between $5 \mathrm{~mm}-15 \mathrm{~mm}$; and a similar dip of smaller magnitude is observed in the $10 \mathrm{~V}$ bias. To assess whether or not this sudden change in bias with respect to WV was a potential cause for the Tb bias anomaly exhibited in Fig. 2.18, we separated the match-up dataset by high and low WV. The results presented in Fig. 2.23 show negligible differences in the bias patterns; hence, water vapor can be rejected as a probable cause. 


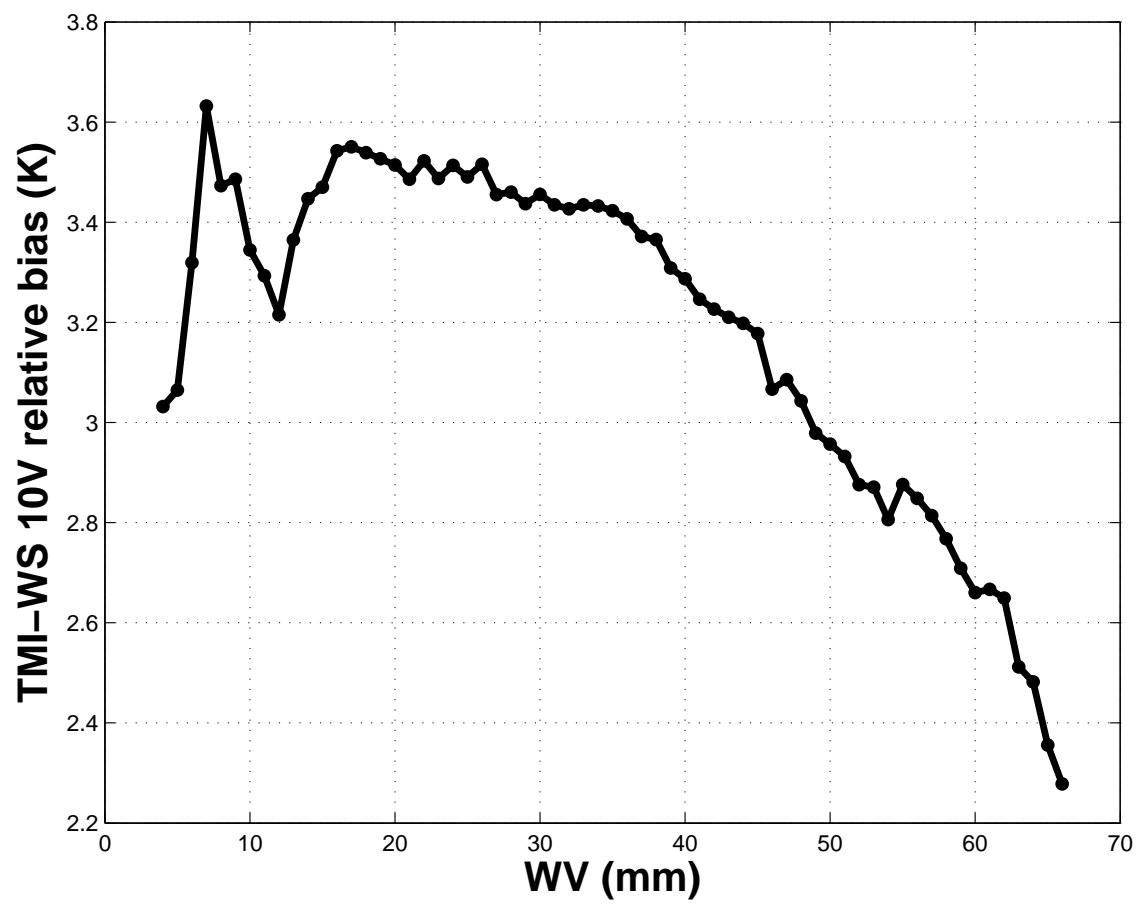

(a) $10 \mathrm{~V}$

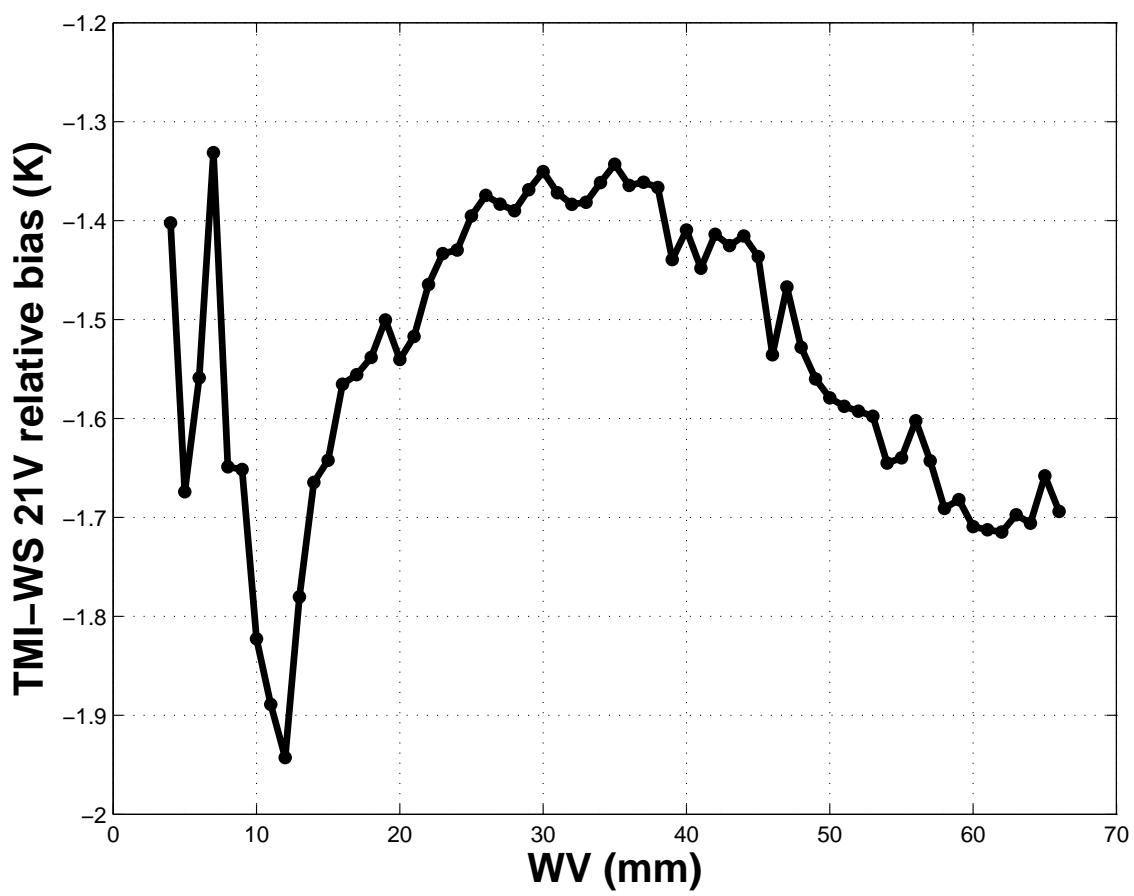

(b) $21 \mathrm{~V}$

Figure 2.22: TMI-WindSat bias dependence on columnar WV. 


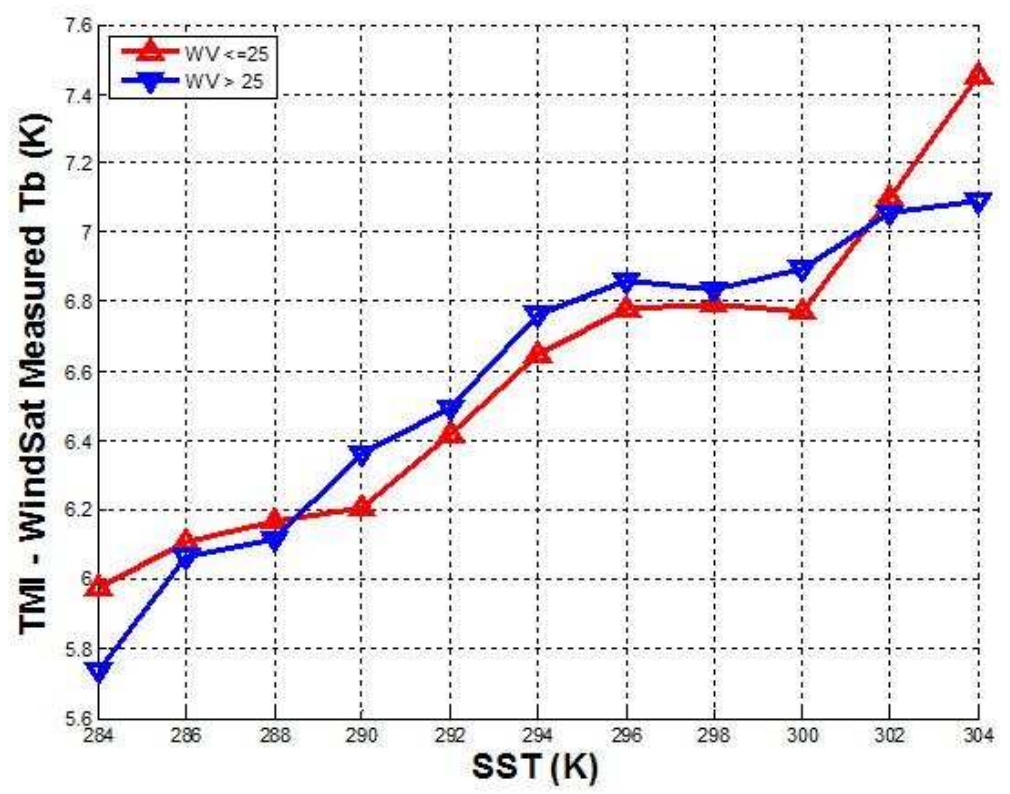

Figure 2.23: WV dependence of TMI-WindSat 10V measured Tb differences.

Finally, the existence of diurnal biases in the GDAS SST values was considered as a possible cause for the step response. Figure 2.24 shows the diurnal variation of SST filtered by different wind speeds $(>2 \mathrm{~m} / \mathrm{s},>5 \mathrm{~m} / \mathrm{s}$ and $>10 \mathrm{~m} / \mathrm{s})$. Since the mean wind speed over the ocean is $\sim 6 \mathrm{~m} / \mathrm{s}$, the diurnal variation of the SSTs is on average $\pm 0.5 \mathrm{~K}$. Since the binned average $\mathrm{Tb}$ biases represent a one-year average, then we should consider that the diurnal cycle is $\sim 1 \mathrm{~K}$ peak to peak. Because the TMI match-ups occur with the sun synchronous satellites, they are separated by 12 hours in the solar cycle (early morning and evening passes). Thus, the $\sim 1 \mathrm{~K}$ diurnal variation in SST is sufficient to account for the observed $10 \mathrm{~V}$ bias change assuming that the TMI passes times were in synchronism with the diurnal cycle. On the other hand, it cannot possible explain such a bias at the higher frequency channels because of their lack of sensitivity to changes in SST. Referring to Fig. 2.17 the sensitivity of the $37 \mathrm{~V}$ channel to SST is $<0.1 \mathrm{~K} / \mathrm{K} \_\mathrm{SST}$, which rules this out as 
the cause of the unexpected bias response. Because of the highly correlated bias patterns in all channels, it is highly unlikely that there is more than one cause for each channel anomaly, which happens to be similar when plotted against the corresponding match-up SST's. Therefore, we reject this hypothesis for the cause of the Tb bias anomaly.

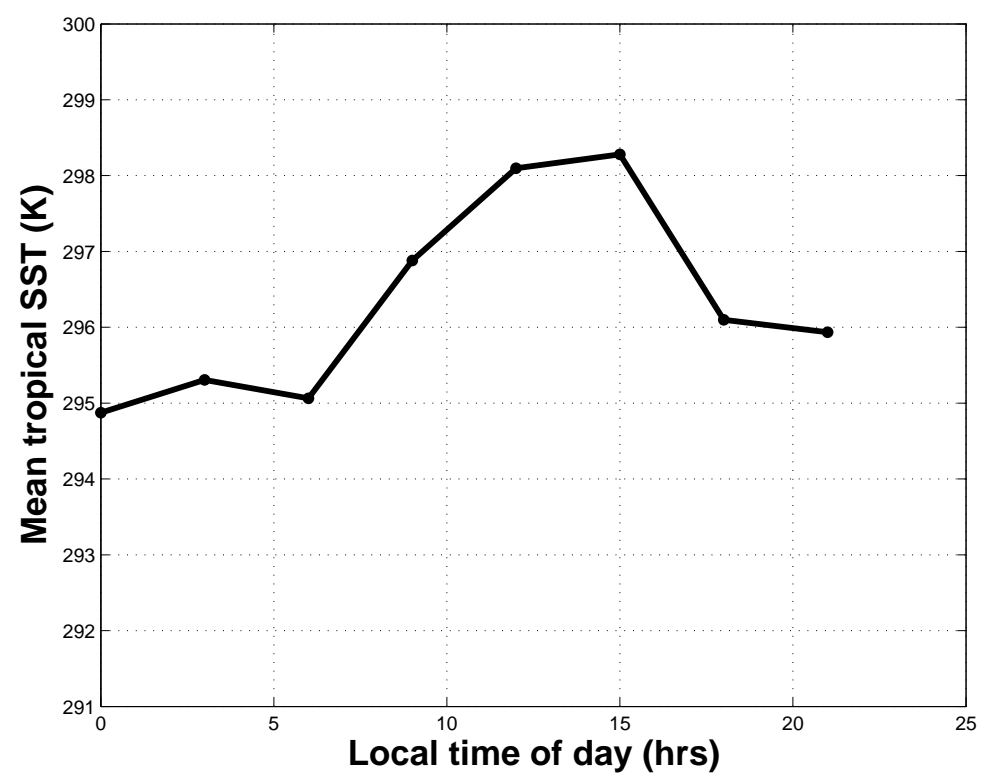

(a) WS $>2 \mathrm{~m} / \mathrm{s}$

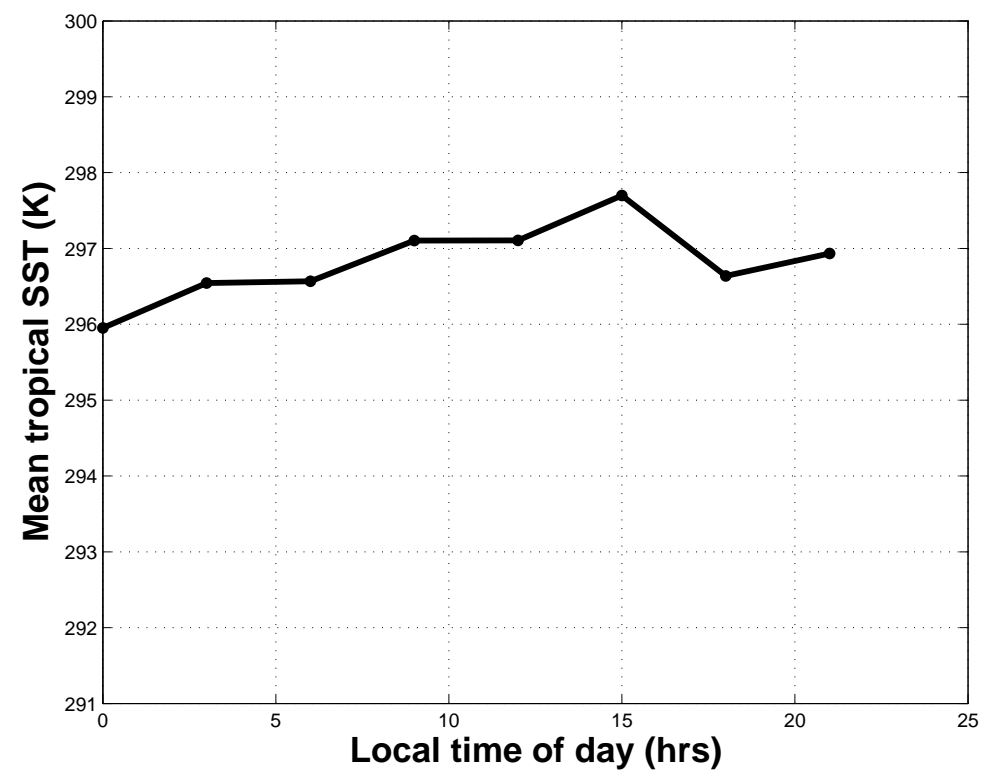

(b) $\mathrm{WS}>5 \mathrm{~m} / \mathrm{s}$

Figure 2.24: GDAS SST diurnal variation. 


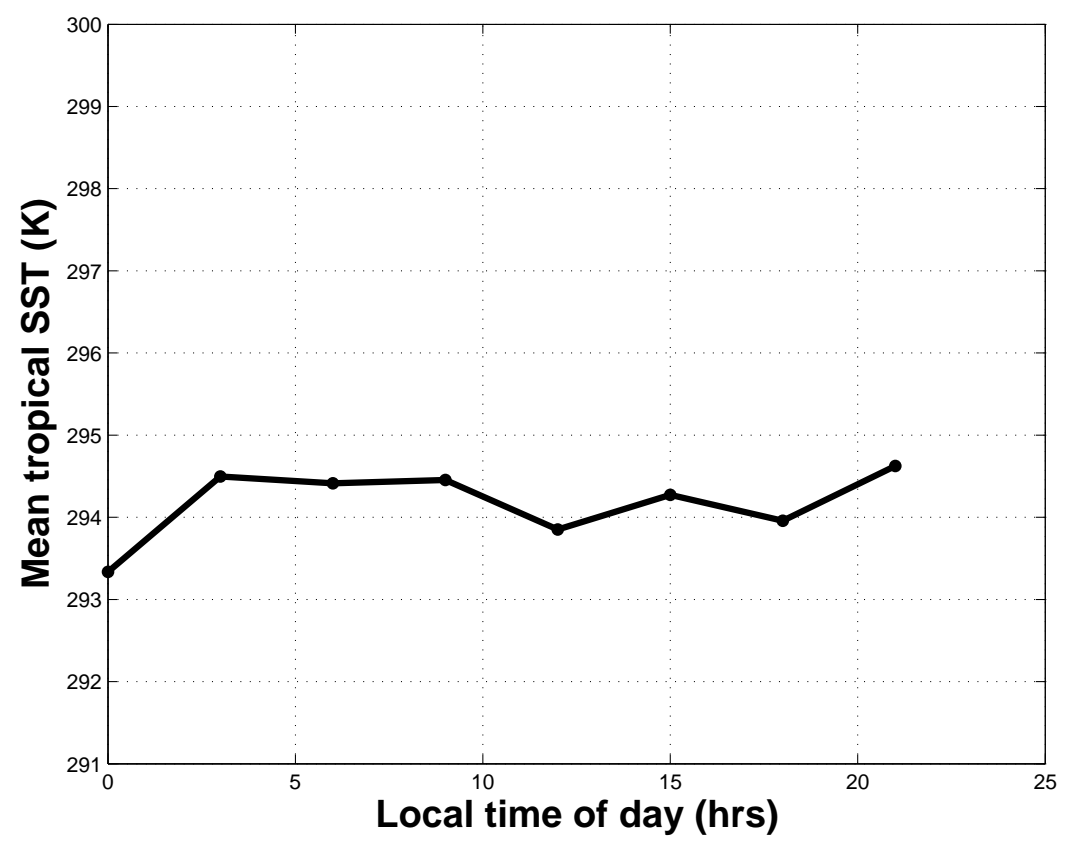

(c) $\mathrm{WS}>10 \mathrm{~m} / \mathrm{s}$

Figure 2.24: GDAS SST diurnal variation.

After eliminating the above as probable causes for the anomalous $\mathrm{Tb}$ biases, we next examined the effects of ascending and descending orbit passes for the two satellites. Separating these match-ups in this manner is equivalent to splitting the TMI observations into two halves of the orbital period i.e., the ascending portion is the first-half of the orbit and the descending is the second. Further, there are ascending and descending portions for the sun-synchronous satellites as well, and these correspond to local solar time of day (ascending passes of the sun-synchronous satellites occur around 6:30 pm and the descending passes occur about 6:30 am). When the match-ups were sorted according to ascending and descending passes (local solar time), we calculated the Tb differences for corresponding 1 degree boxes for TMI with itself and similarly for WindSat with itself i.e., we calculated the difference between TMI morning and evening match-ups and the same for WindSat. 
This resulted in a surprising discovery as shown in Fig. 2.25. Here the average of all TMI Tb measurements for the ascending passes were $\sim 1.5 \mathrm{~K}$ higher than those of the descending passes, while the ascending and descending pass Tb values were almost equal for WindSat. Since the collocation boxes were identical for TMI and WindSat, this meant that TMI was different from WindSat; and we had to determine which was correct.

Certainly, we expect that there should be some small diurnal $\mathrm{Tb}$ difference $(\leq 0.5 \mathrm{~K})$ caused by the SST diurnal cycle; but this should be nearly the same for both TMI and WindSat. By examining the theoretical Tb's calculated by the RTM, we found that both the TMI modeled Tb's and the WindSat modeled Tb's at their respective frequencies and incidence angles had near zero differences between ascending and descending passes. Further, this is also consistent with the observed histograms of TMI to WindSat unexplained Tb biases shown in Fig. 2.26, where the 10V Tb bias for evening and morning passes are uni-modal, as expected. Further, similar time-varying biases were consistently observed for all TMI channels, and the results are shown in Appendix-C.

Given this, there was only one possible explanation, which suggested that the TMI radiometric calibration was varying in time. Considering that TMI has been successfully operating on-orbit for over a decade and that many engineers and scientists have used these 1B11 $\mathrm{Tb}$ data in their research without any recorded findings of anomalies, makes this an extremely unlikely cause. Nevertheless, it is the only conclusion that we can make; and as will be shown in the remainder of this dissertation, there is now compelling evidence that this is true. 


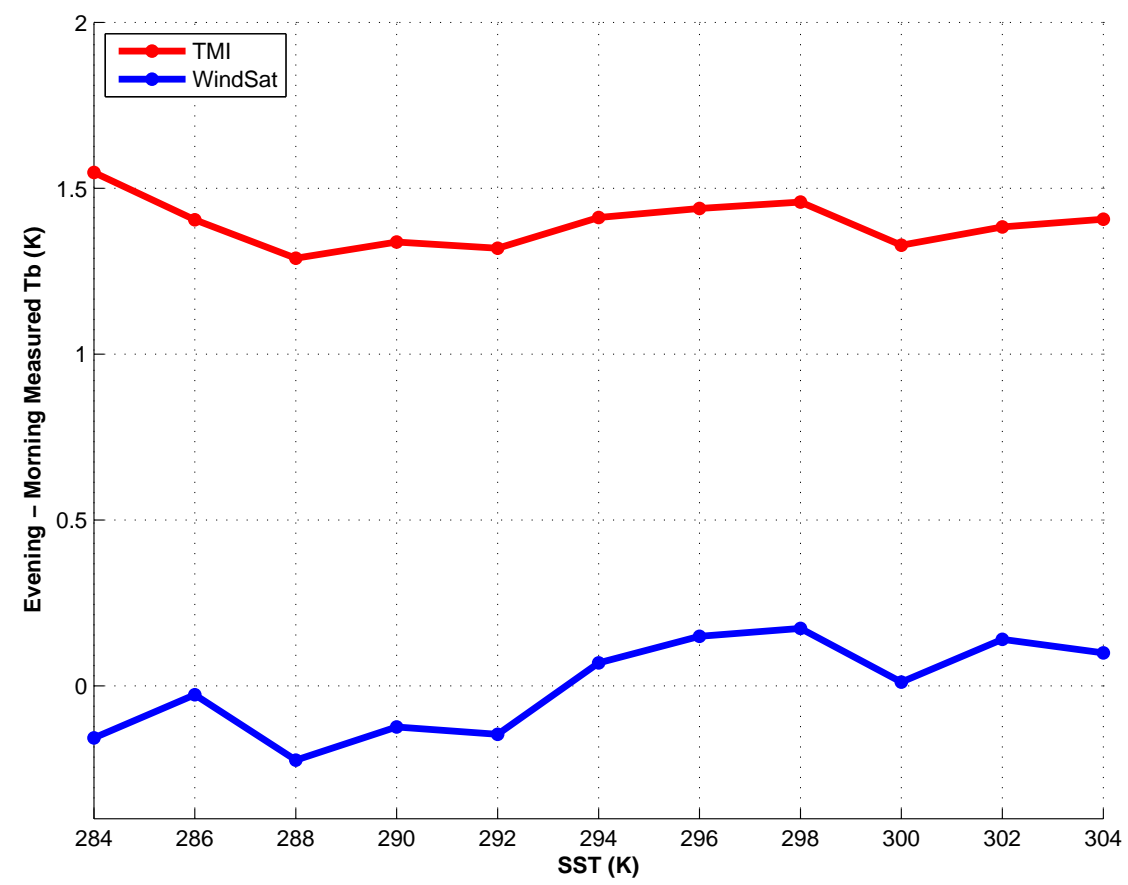

Figure 2.25: Evening - Morning measured Tb differences separately for TMI and WindSat. 


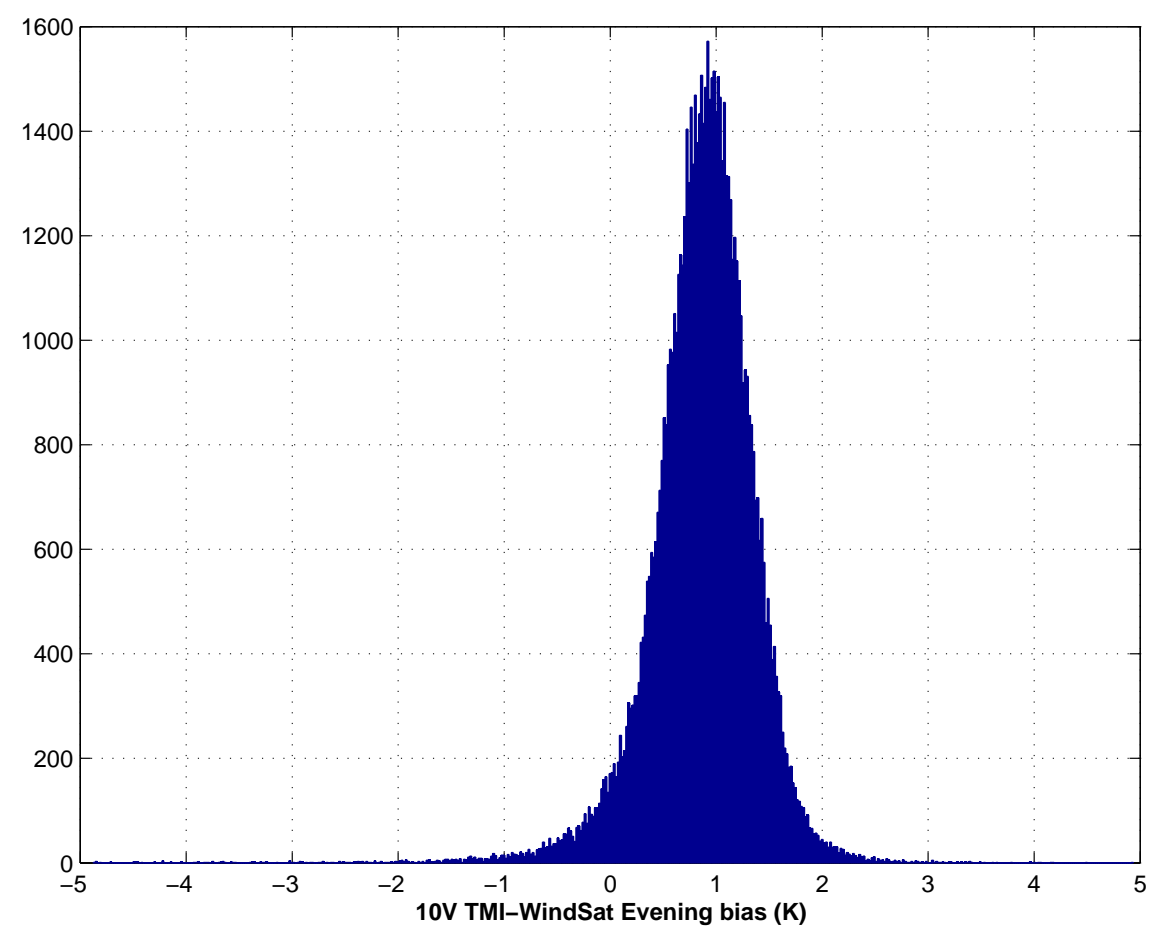

(a) Evening

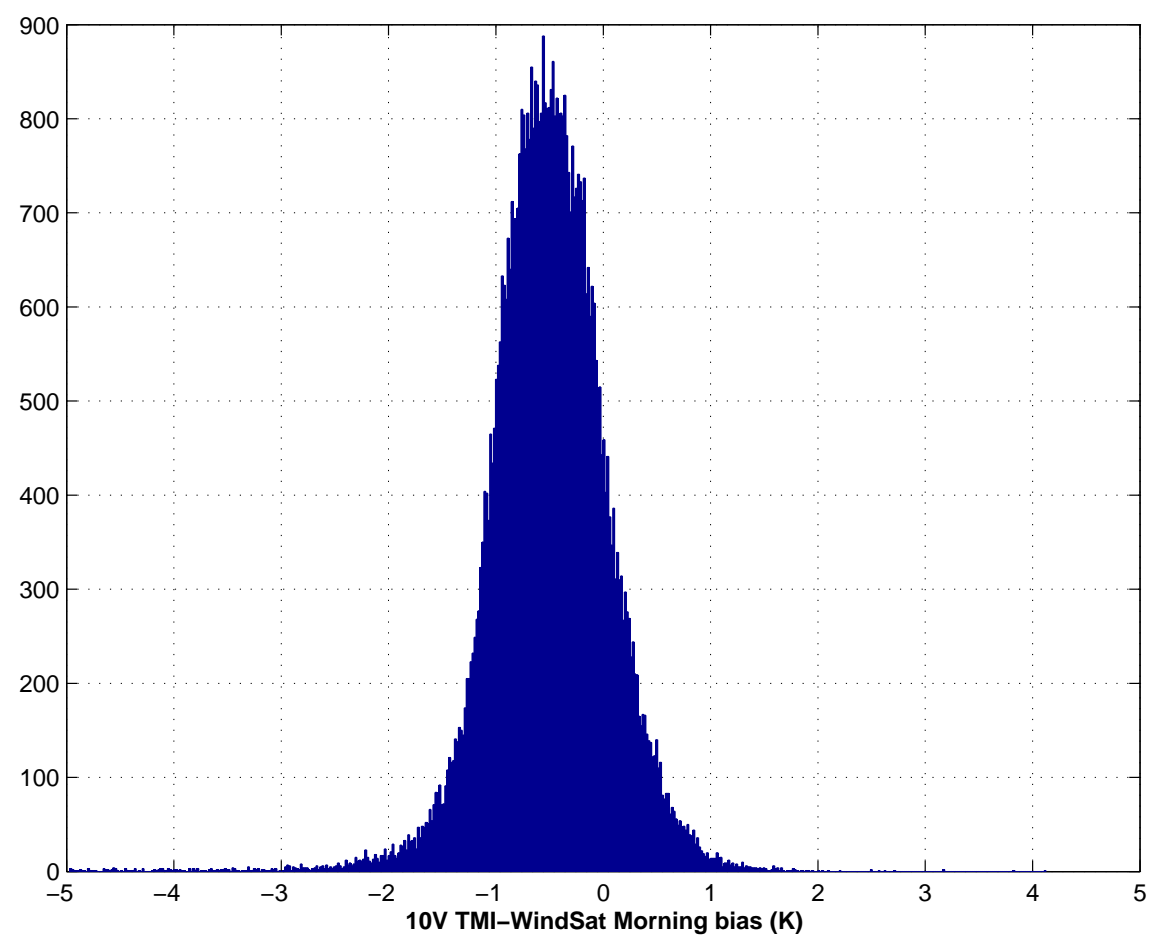

(b) Morning

Figure 2.26: Evening and Morning TMI - WindSat radiometric bias histograms. 
To confirm that this observation was independent of WindSat, similar match-ups were performed and radiometric biases calculated for TMI comparisons with two independent SSMI instruments (F-13 and F-14); and the results shown for the 37V channel in Fig. 2.27 support the same conclusion as WindSat. Further, these results for $37 \mathrm{~V}$ are representative of those for the other TMI channel comparisons with SSMI. Thus, TMI exhibits a consistent variable radiometric calibration compared to WindSat and the two SSMI's that varies with solar local time of day. Further, it is significant to note that the relative $\mathrm{Tb}$ biases for the $37 \mathrm{~V}$ channels are different for the different satellites; yet all exhibit excellent stability $(\sim \pm 0.25 \mathrm{~K})$ over the one-year ICWG period of comparisons. This implies that all these radiometers (including TMI) have stable calibrations when averaged over a monthly period; but the calibrations are not equal based upon the different bias values for each satellite. Note that the $37 \mathrm{~V}$ biases are smaller for the two SSMI radiometers than for WindSat; but this is not consistent for all channels. Table 2.5 shows the one-year mean relative biases between TMI and WindSat/SSMI for the 10 - $37 \mathrm{GHz}$ channels.

It is important to note that these biases are the adjustment required to bring the various radiometer channels into agreement, using the TMI as the secondary calibration standard. Unfortunately, it does NOT imply that TMI has the "best" radiometric calibration nor that the other radiometers are inferior. 


\section{TMI/SSMI F-13 37V}

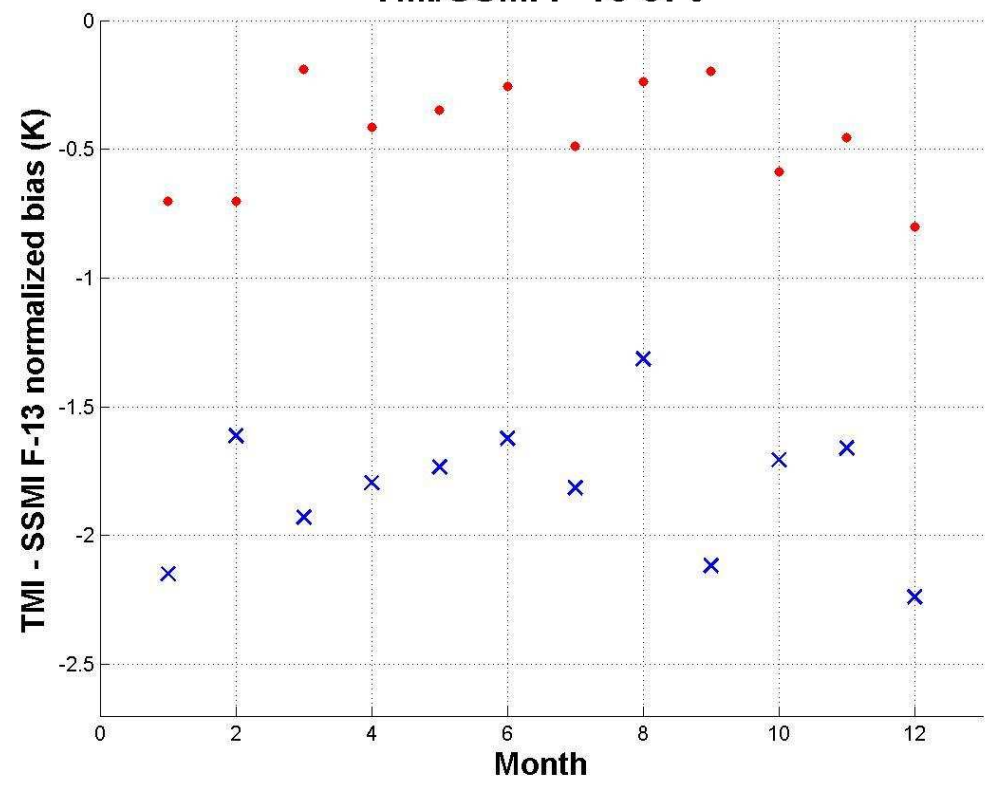

(a) SSMI F-13.

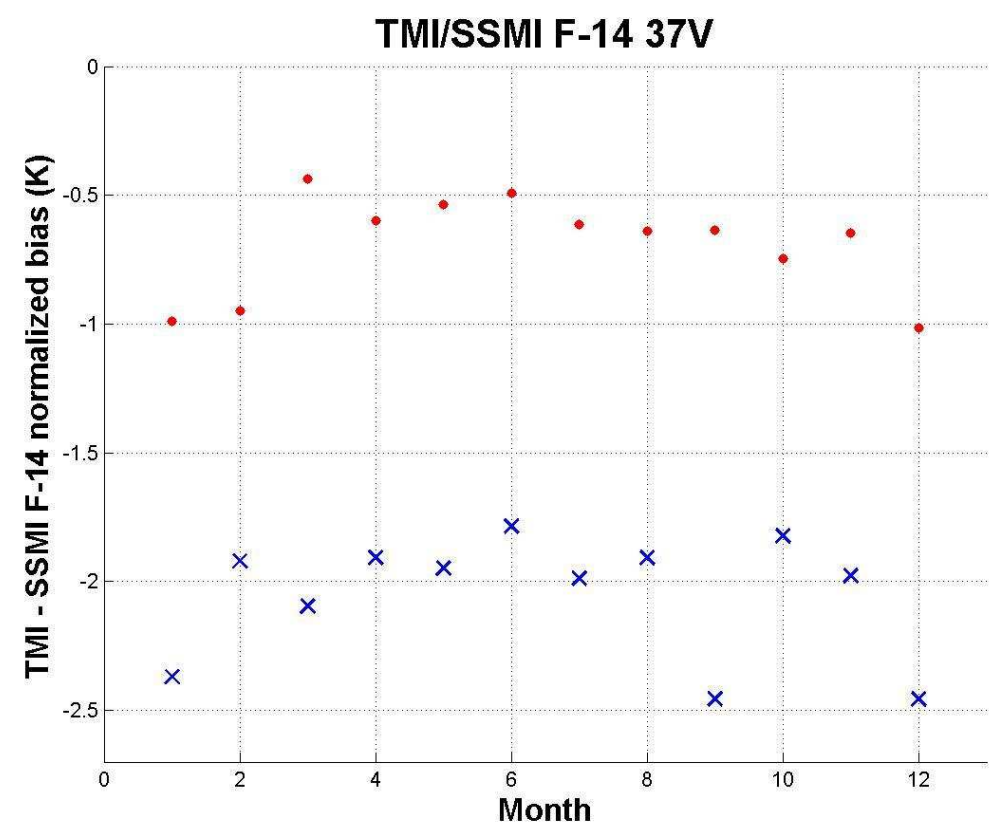

(b) SSMI F-14.

Figure 2.27: Radiometric calibration differences for Evening and morning collocations between TMI and WindSat/SSMI. The monthly averages given as red "dot' symbols are evening And the blue "X" symbols are morning. 
TMI/WindSat 37V

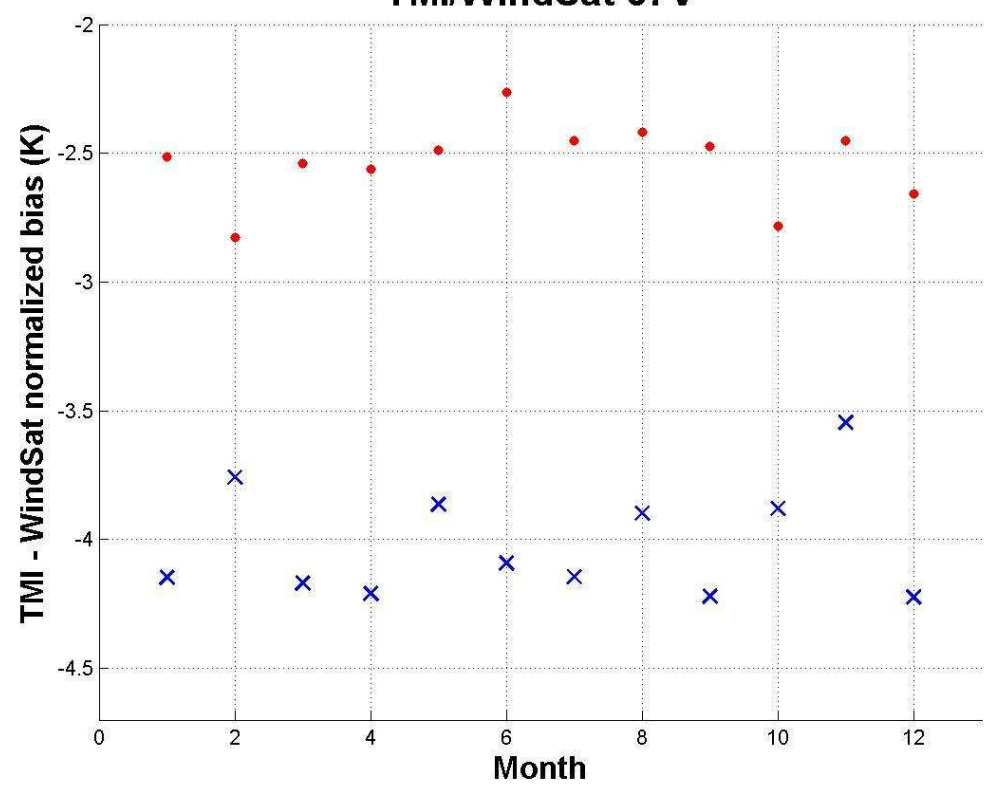

(c) WindSat

Figure 2.27: Radiometric calibration differences for Evening and morning collocations between TMI and WindSat/SSMI. The monthly averages given as red 'dot' symbols are evening and the blue ' $\mathrm{X}$ ' symbols are morning.

Table 2.5: Relative bias between TMI and WindSat/SSMI

\begin{tabular}{|c|c|c|c|c|c|c|}
\hline \multirow{2}{*}{ Channel } & \multicolumn{2}{|c|}{ TMI - F13 } & \multicolumn{2}{c|}{ TMI - F14 } & \multicolumn{2}{c|}{ TMI - WindSat } \\
\cline { 2 - 7 } & Evening & Morning & Evening & Morning & Evening & Morning \\
\hline $10 \mathrm{~V}$ & - & - & - & - & 0.87 & -0.54 \\
\hline $10 \mathrm{H}$ & - & - & - & - & -1.21 & -2.23 \\
\hline $19 \mathrm{~V}$ & -0.44 & -1.86 & -0.07 & -1.43 & 0.16 & -1.38 \\
\hline $19 \mathrm{H}$ & -1.45 & -2.74 & -1.26 & -2.58 & -2.81 & -3.89 \\
\hline $21 \mathrm{~V}$ & -1.61 & -2.99 & -1.23 & -2.62 & -0.95 & -2.58 \\
\hline $37 \mathrm{~V}$ & -0.44 & -1.82 & -0.68 & -2.06 & -2.53 & -4.01 \\
\hline $37 \mathrm{H}$ & -1.54 & -2.77 & -1.36 & -2.56 & -1.92 & -3.18 \\
\hline
\end{tabular}


Finally, we will revisit these relative time-of-day biases in Chapter 3 of this dissertation, where we present a detailed description of a parametric orbital time and day of the year based correction to compensate for variable radiometric calibration. We will present results, which demonstrate that the empirical correction (derived under this dissertation) effectively "stabilizes" the TMI variable radiometric calibration. 


\section{TMI ORBITAL BIAS MODELING}

The time-varying radiometric calibration of the TMI brightness temperatures, which was confirmed by the inter-calibration analysis described in the previous chapter, directly impacts the accuracy of the geophysical retrievals made using TMI observations. Chapter 2 provided evidence that the TMI radiometric calibration varies systematically based on the time of day and that the TMI observations in the evening were biased high by $\sim 1.5 \mathrm{~K}$ compared to the observations in the morning. The purpose of this chapter is to examine the cause of this time-variable radiometric calibration and to find an engineering solution to "fix it". We start with the hypothesis that the time-variable radiometric calibration is due solely to the instantaneous physical temperature of the reflector. Our testing of this hypothesis and conclusions follow.

Since the TRMM spacecraft flies in a non-sun-synchronous orbit, it has a sun-lighted portion $(\sim 60 \mathrm{~min})$ and a night portion (eclipse $\sim 33 \mathrm{~min})$. The time of eclipse (start time and duration) changes daily on approximately a 47-day cycle, which is the TRMM orbit precession cycle. During this orbit precession cycle, the angle of the sun with respect to the TRMM orbital plane, known as the solar beta angle, changes daily and after 46.7 days it repeats. Also the angle of the "line-of-sight" between the Earth and sun relative to the equatorial plane of the Earth varies seasonally (period 365.25 days). Thus, the geometry associated with the TMI solar heating during the sun-lighted portion is a rather complicated product of these two sinusoidal cycles. Unfortunately, because these periods are not integer multiples, there is not an exact repeating pattern; therefore, one should expect that the reflector temperature profile for any day is unique. 
As described in Section 1.2, during the TMI commissioning after launch, a larger than expected Tb calibration bias was discovered by the TRMM TMI instrument sub-team. This anomalous calibration cause was attributed to a slightly emissive reflector bias that was discovered in the TMI post-launch calibration and documented by Wentz et al. [7]. Subsequently, Wentz developed an additive offset $\mathrm{Tb}$ correction, which was implemented by the TRMM Science Data Information System (TSDIS) and used in the TMI brightness temperature product 1B11.

Following our time-variable radiometric calibration discovery, we revisited this emissive reflector bias as a possible cause for the time-varying TMI calibration revealed by the inter-calibration analysis. The TMI 1B11 data product contains a correction of $\sim 10 \mathrm{~K}$ for the reflector bias; however, this correction uses a mean (constant) physical temperature for the reflector temperature, because the instantaneous physical temperature of the TMI reflector is unknown (i.e., not measured). Unfortunately, the ramifications of this correction were not recognized because the peak-to-peak variation of the reflector temperature over an orbit is estimated to be as high as $80 \mathrm{~K}$ [7]; thus, with a reflector emissivity of $\sim 3.5 \%$, the peak-to-peak brightness temperature bias change over an orbit can be $\sim 3 \mathrm{~K}$. The timevarying calibration bias over one-orbit of $1.5 \mathrm{~K}$ revealed by the inter-calibration analysis is well within these limits.

Since TRMM flies in a non-sun-synchronous orbit, the TMI reflector temperature varies in a cyclic manner each orbit as it is alternatively heated by the sun during the daylight portion and radiatively cooled during the night. Compared to the orbit average temperature, the instantaneous temperature of the TMI reflector is expected to be warmer 
when the local time of the observations is $\sim 6: 30 \mathrm{pm}$ (1830 hours), and the reason for this is illustrated in Fig. 3.1 for TRMM orbit number 43829 on July 25, 2005. This figure plots the local time of day for the center pixel of the swath versus TMI scan number for one orbit period. The scans during which TRMM is illuminated by sunlight are plotted in red, and the scans during which the TRMM flies in the earth's shadow (eclipse) are plotted in blue. When observations are made at 6:30 pm local time, TRMM is exposed to sunlight for a period of about 1800 scans ( $\sim 57$ minutes out of $\sim 92$ minutes orbital period) for this day. In contrast, for the observations made at 6:30 am, TRMM is exposed to sunlight only for $\sim 250$ scans (8 minutes); and as a result, the reflector physical temperature is expected to be warmer for observations made at 6:30 pm than at 6:30 am. Since the actual additive emissive reflector bias is directly proportional to the reflector physical temperature, the resulting calibration bias is also expected to be higher in the evening than in the morning. Thus, the time of day dependent variation in the inter-calibration results between TMI and WindSat/SSMI is consistent with the expected variation of the emissive reflector bias. Further results presented later in this chapter confirm this hypothesis that the time-varying radiometric calibration is caused by TMI's slightly emissive reflector and its on-orbit physical temperature cycle. 


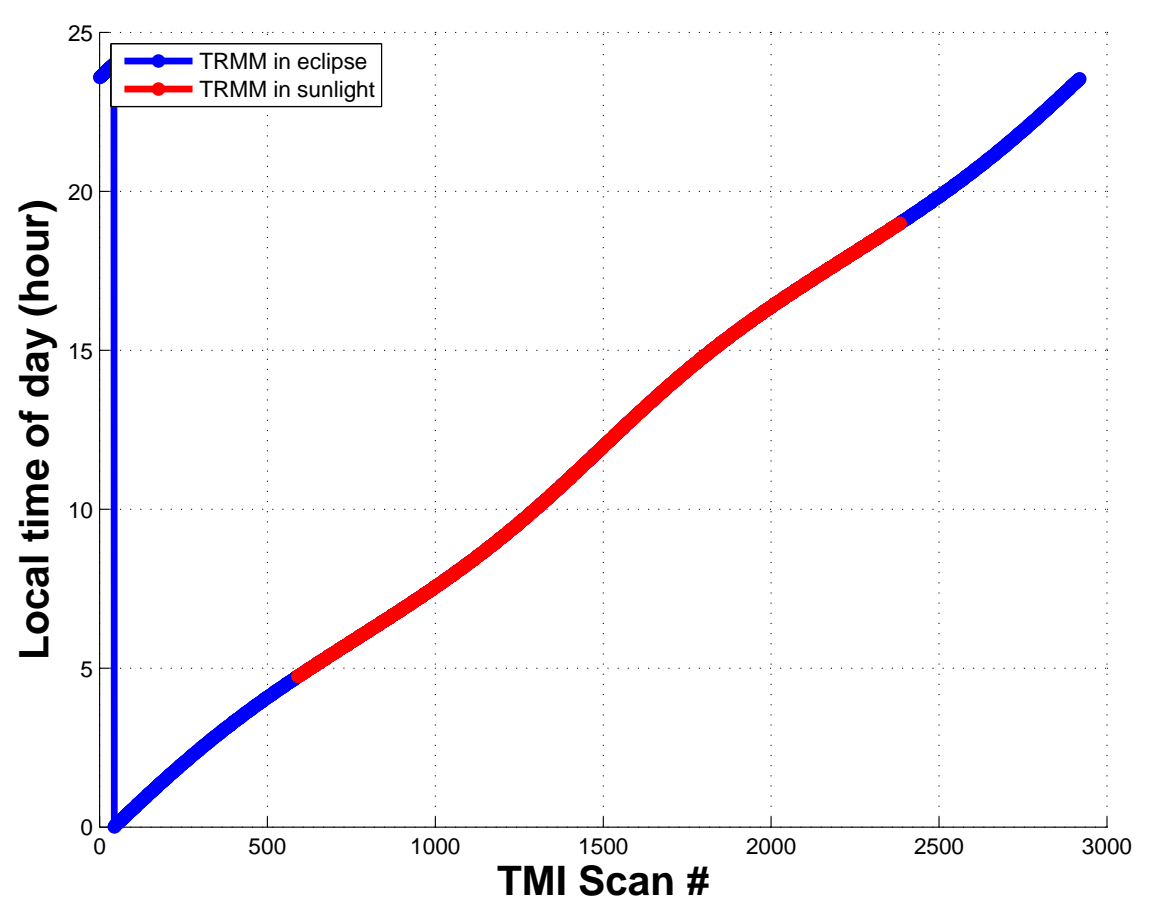

Figure 3.1: Local time of day variation over a typical TMI orbit.

The remainder of this chapter describes an empirical method used to determine and to correct the TMI Tb bias variation due to the reflector temperature changes over an orbit. This ensures that the TMI calibration remains stable and avoids potential systematic errors in the geophysical retrievals made from TMI observations. First, Section 3.1 describes the modeling procedure used to estimate the TMI reflector bias. Then, Section 3.2 describes the orbital averaging performed in order to estimate the variation of the reflector temperature over the course of an orbit. Finally, Section 3.3 provides evidence that the proposed empirical correction effectively eliminates the time-varying TMI radiometric bias. 


\subsection{TMI Tb Modeling}

Since WindSat and SSMI are sun-synchronous satellites, they have constant (and similar) local times for ascending and descending observations, and for any given day, the match-ups of TMI observations with WindSat and SSMI provide only two time samples in the orbital cycle of the TMI calibration error. Characterization of the TMI orbital radiometric bias using match-ups with polar orbiting satellites would therefore require the use of many such satellites with different nodal crossing times. Unfortunately, it is likely that such a group of polar orbiting satellites would have different calibration errors, which would significantly complicate the estimation of the time-varying orbital cycle in the TMI Tb bias.

Therefore, this dissertation uses an alternative approach of comparing rain-free ocean observations across the TMI swath with the brightness temperature (Tb) predictions from radiative transfer modeling for the corresponding ocean scenes. This approach is complementary to the match-up approach and will make it possible to fill in portions of the measurement space not well covered by the sun-synchronous satellite radiometer match-ups. The TMI observations are averaged into $1^{\circ}$ latitude by longitude boxes and $\mathrm{Tb}$ predictions are generated for each such $1^{\circ}$ box using the ICWG consensus radiative transfer model (RTM), which is described later in this section. Following the conclusions of Wentz et al. [7], we assume that the TMI reflector emissivity is spectrally invariant (independent of channel frequency); therefore, the orbital $\mathrm{Tb}$ bias can be calculated by making comparisons between measured and modeled Tb's for a single TMI channel. 


\subsubsection{TMI Channel Selection}

We pick the $10 \mathrm{GHz}$ vertically polarized (10V) channel, because it has a small average atmospheric component of brightness temperature $(\sim 10 \mathrm{~K})$ and is, therefore, primarily sensitive to the ocean surface emission. This has the desirable effect of minimizing the radiative transfer (theoretical) modeling uncertainty. The emissivity of a calm ocean surface depends on the dielectric constant of sea water and Fresnel reflection coefficients, both of which are well known. In addition, for oceanic wind speeds less than $10 \mathrm{~m} / \mathrm{s}$, the effect of surface roughness and foam from breaking ocean waves on the ocean emissivity is well understood and contributes less than $1 \mathrm{~K}$ to the $\mathrm{V}$-pol $\mathrm{Tb}$ at the $\mathrm{TMI} 53^{\circ}$ incidence angle.

Further, the V-pol surface emission is much less sensitive than the H-pol to changes in the wind speed at the TMI incidence angle. To illustrate this, a theoretical RTM calculation of the $10 \mathrm{GHz}, \mathrm{V}$-pol and H-pol surface emissions versus incidence angle for a typical oceanic scene with SST of $300 \mathrm{~K}$, salinity of $30 \mathrm{ppt}$, and for wind speeds of $0 \mathrm{~m} / \mathrm{s}, 5 \mathrm{~m} / \mathrm{s}$ and $10 \mathrm{~m} / \mathrm{s}$ is shown in Fig. 3.2. The surface emission increases for higher wind speeds from $163.5 \mathrm{~K}$ at $0 \mathrm{~m} / \mathrm{s}$ to $164.4 \mathrm{~K}$ at $10 \mathrm{~m} / \mathrm{s}$; thus, the variation of the surface emission for the TMI incidence angle (53 deg) is less than $1 \mathrm{~K}$ for the 3 cases. In contrast, the H-pol surface emissions are more sensitive to changes in wind speeds as shown in Fig. 3.3. For this case, the H-pol surface emissions extend from slightly greater than $74 \mathrm{~K}$ at $0 \mathrm{~m} / \mathrm{s}$ to almost $83 \mathrm{~K}$ at $10 \mathrm{~m} / \mathrm{s}$ at the $53^{\circ}$ incidence angle; therefore, the sensitivity of the $\mathrm{H}$-pol surface emissions to wind speed is approximately 8 times greater than that of V-pol. This relative insensitivity of the $10 \mathrm{~V}$ channel to wind speed is a major factor in our decision to use this channel to estimate the TMI calibration bias. 


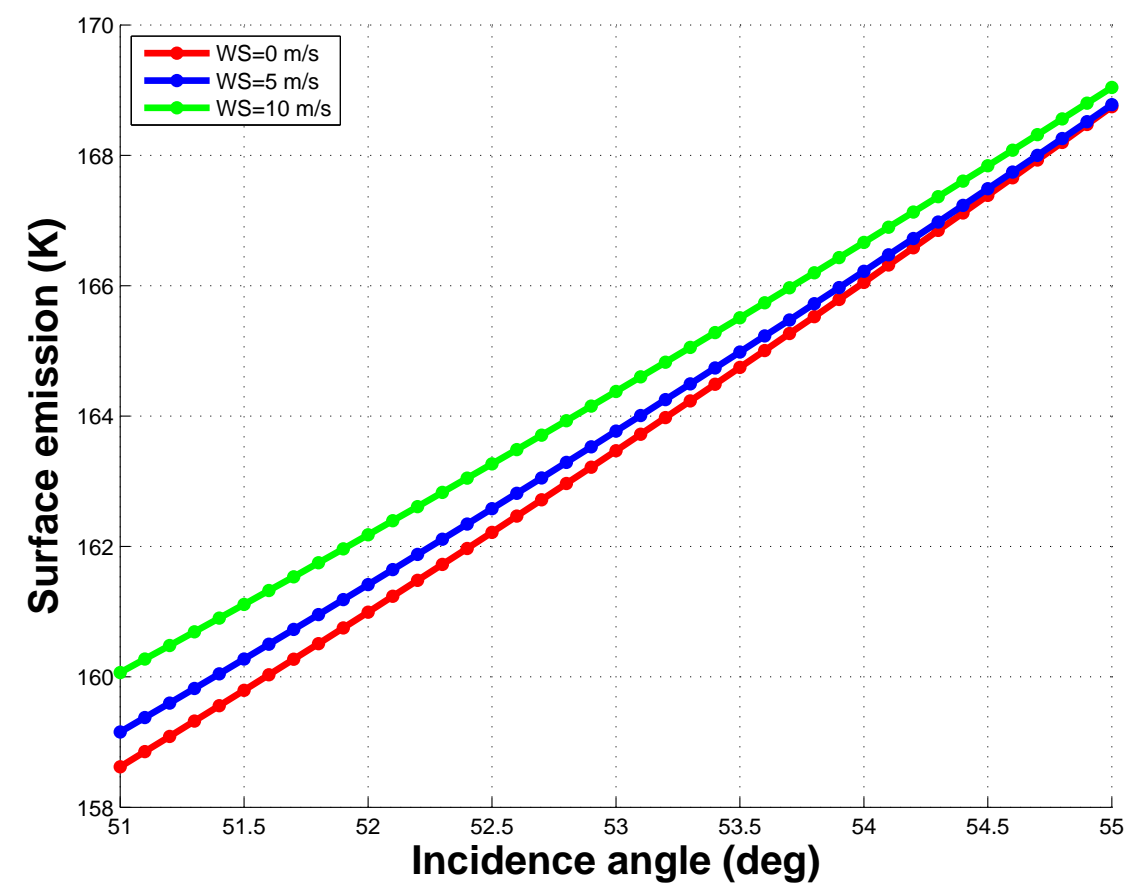

Figure 3.2: Incidence angle dependence of $10 \mathrm{~V}$ surface emission.

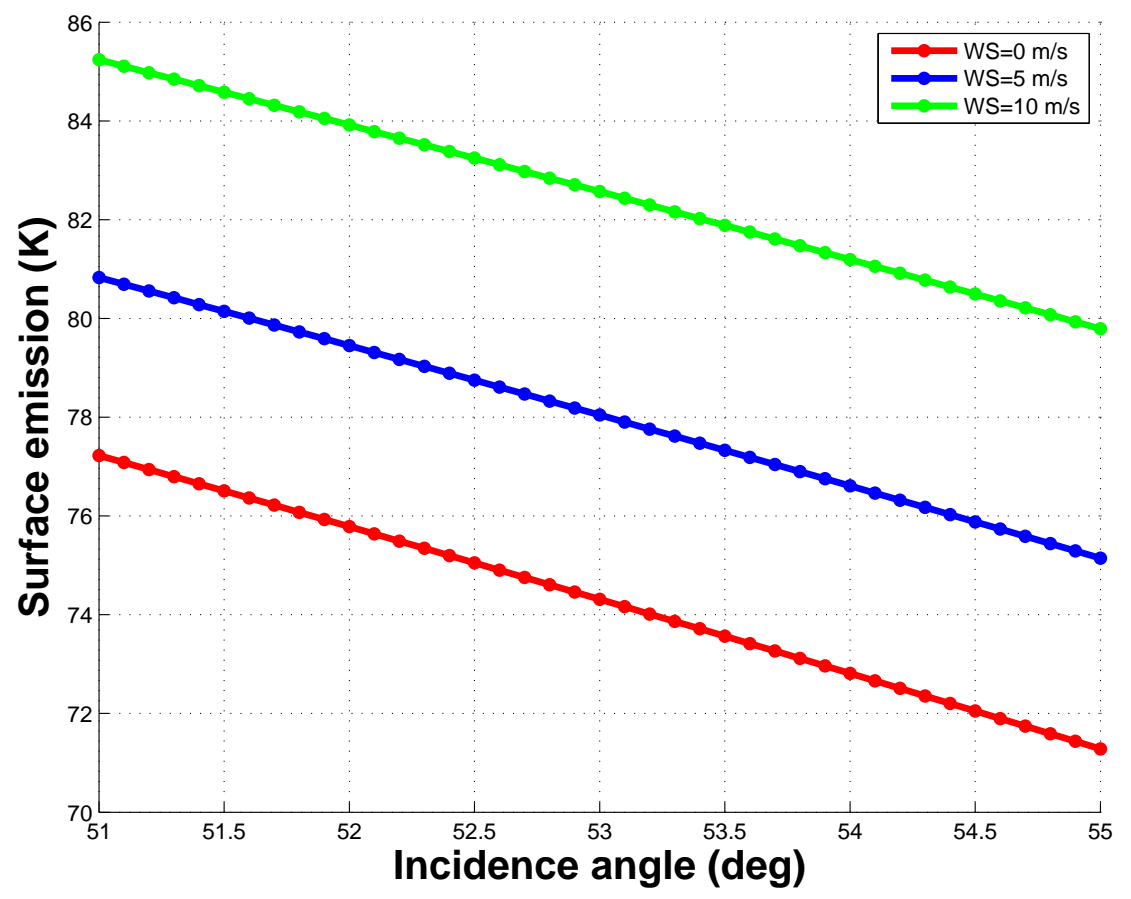

Figure 3.3: Incidence angle dependence of $10 \mathrm{H}$ surface emission. 
Another important advantage of using the $10 \mathrm{~V}$ channel over the $10 \mathrm{H}$ channel is its reduced sensitivity to changes in atmospheric parameters (e.g., water vapor and cloud liquid water). The RTM calculations are performed using atmospheric parameters extracted from GDAS, whose quantization is coarse relative to the TMI radiometer footprint. Since the atmospheric parameters such as water vapor and cloud liquid water are expected to be slightly inhomogeneous over a $1^{\circ}$ box; the atmospheric profiles extracted from GDAS are likely to provide a slightly distorted estimate of the atmospheric conditions in the TMI observation's view path. Thus, it is desirable for calibration purposes that the observed $\mathrm{Tb}$ for a given channel be insensitive to small changes in atmospheric conditions. As seen from Figures 3.2 and 3.3, the vertical polarization has a greater surface emissivity than the horizontal polarization for the $10.7 \mathrm{GHz}$ frequency. The power reflection coefficient $(\Gamma)$ of the surface is related to the surface emissivity as :

$$
\Gamma=(1-\epsilon)
$$

Since the V-pol has a higher emissivity than the H-pol, the V-pol power reflection coefficient is lower; thus, the ocean surface reflects a smaller fraction of the downward atmospheric emission incident on the surface (which is randomly polarized) at the V-pol when compared to the H-pol. This is illustrated in Fig. 3.4 which contains a simulation of the reflected Tb's from the surface for an ensemble of 63 atmospheric profiles generated by Wilheit [33]. The ensemble consists of 7 handbook atmospheres (U.S. Standard, Tropical, Subtropical Summer, Subtropical Winter, Midlatitude Summer, Midlatitude Winter, and Subarctic Summer). A set of 9 different cloud models, which are listed in Table 3.1, is injected into 
each of the atmospheres, thereby creating a set of 63 profiles. The surface scene was assumed to have a SST of $300 \mathrm{~K}$, wind speed of $10 \mathrm{~m} / \mathrm{s}$ and salinity of $30 \mathrm{ppt}$. The reflected $\mathrm{Tb}$ varies from $4 \mathrm{~K}$ to $10 \mathrm{~K}$ for the $\mathrm{V}$-pol, while it varies from $6 \mathrm{~K}$ to $16 \mathrm{~K}$ for the $\mathrm{H}$-pol.



Figure 3.4: Reflected downwelling atmospheric Tb's for an ensemble of 63 profiles for the $10 \mathrm{~V}$ and $10 \mathrm{H}$ channels.

Table 3.1: Cloud models

\begin{tabular}{|c|c|c|c|c|}
\hline Model number & Cloud Base $(\mathrm{km})$ & Cloud Top $(\mathrm{km})$ & Density $(\mathrm{gm} / \mathrm{m} 3)$ & CLW $(\mathrm{mg} / \mathrm{cm} 2)$ \\
\hline 1 & 1 & 2 & 0.1 & 10 \\
\hline 2 & 1 & 2 & 0.3 & 30 \\
\hline 3 & 0 & 8 & 0.1 & 80 \\
\hline 4 & 7 & 8 & 0.2 & 20 \\
\hline 5 & 1 & 3 & 0.04 & 8 \\
\hline 6 & 1 & 5 & 0.04 & 16 \\
\hline 7 & 2 & 4 & 0.02 & 40 \\
\hline 8 & 6 & 8 & 0.2 & 0 \\
\hline 9 & - & - & 0 & \\
\hline
\end{tabular}


Finally, the $10 \mathrm{GHz}$ frequency channel is preferred to the higher frequency channels because it is not as sensitive to variations in atmospheric parameters such as water vapor and cloud liquid water. Fig. 3.5 shows the apparent brightness temperatures for each of the 63 atmospheric profiles described above, for the $10 \mathrm{~V}, 19 \mathrm{~V}, 21 \mathrm{~V}$ and $37 \mathrm{~V}$ channels. A standard oceanic surface scene with $\mathrm{SST}=300 \mathrm{~K}$, wind speed $=10 \mathrm{~m} / \mathrm{s}$ and salinity $=$ $30 \mathrm{ppt}$ was assumed. The $10 \mathrm{~V}$ Tb has a peak to peak variation of $\sim 10 \mathrm{~K}$ over the entire ensemble, while the Tb's of the other channels have peak-to-peak variations of close to $50 \mathrm{~K}$. Further, the effects of cloud liquid water and the other atmospheric parameters pressure, temperature and water vapor (which are relatively more correlated with each other) on the modeled Tb's were considered separately. Fig. 3.6 shows the simulation of the $10 \mathrm{~V}, 19 \mathrm{~V}$, $21 \mathrm{~V}$ and 37V channel Tb's for the 9 different cloud liquid water profiles, assuming constant values for the other atmospheric parameters. The $10 \mathrm{~V}$ channel has a peak-to-peak variation of less than $10 \mathrm{~K}$ while the other channels have a peak-to-peak variation of between $20 \mathrm{~K}$ and 30K. Fig. 3.7 shows the simulation of the 10V, 19V, 21V and $37 \mathrm{~V}$ channel Tb's for the 7 different handbook atmosphere profiles, assuming a constant value of $10 \mathrm{gm} / \mathrm{cm} 2$ for CLW. As expected, the $21 \mathrm{~V}$ water vapor channel has the largest peak-to-peak variation of almost $50 \mathrm{~K}$ while the $19 \mathrm{~V}$ and $37 \mathrm{~V}$ channels have a peak-to-peak variation of between $20 \mathrm{~K}$ and $30 \mathrm{~K}$. However, the peak-to-peak variation for the $10 \mathrm{~V}$ channel is less than $5 \mathrm{~K}$. Thus, given the uncertainty in our knowledge of the atmospheric inputs to the RTM, the $10 \mathrm{~V}$ channel is the most desirable to use in the TMI Tb bias modeling. 




Figure 3.5: Modeled ocean apparent $\mathrm{Tb}$ for an ensemble of 63 atmospheric profiles.

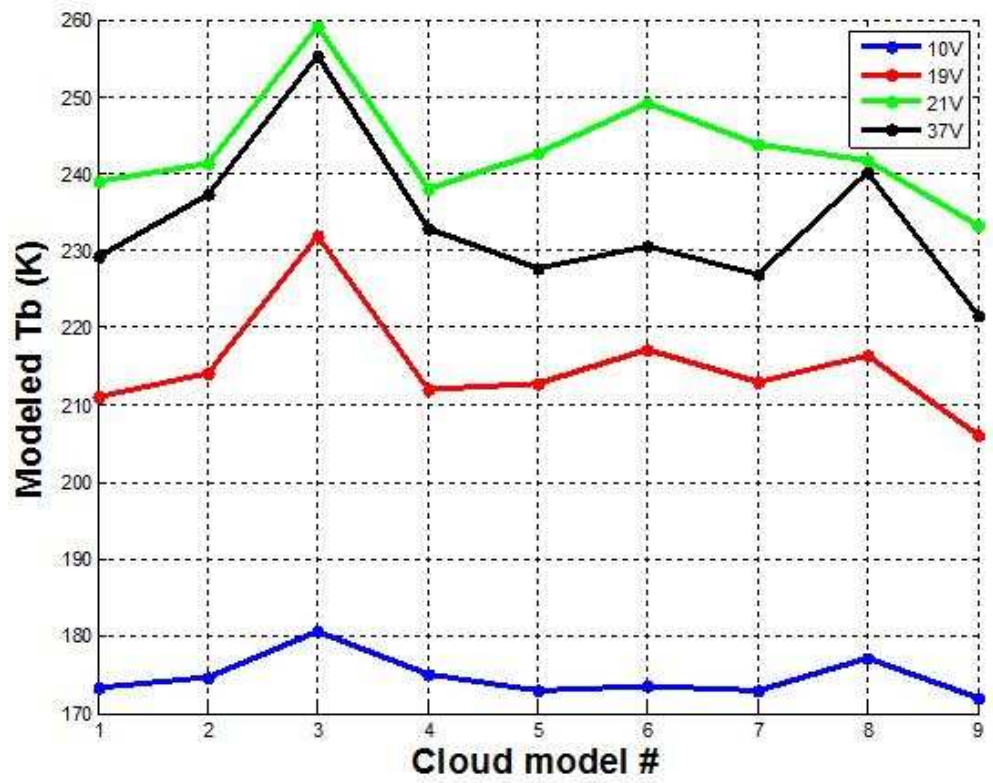

Figure 3.6: Modeled ocean apparent $\mathrm{Tb}$ for an ensemble of 9 cloud models. 




Figure 3.7: Modeled ocean apparent Tb for 7 handbook atmospheres. 


\subsubsection{Typical Environmental Parameters in 1 deg Boxes}

To illustrate the typical range of environmental parameters observed over the rainfree ICWG data set, we present Fig. 3.8, which shows the histograms of the wind speeds, columnar water vapor and cloud liquid water for the rain-free TMI ocean observations for July 2005. For surface wind speed, the ocean scenes have a Rayleigh probability distribution function (pdf) with an average of about $6.6 \mathrm{~m} / \mathrm{s}$ and greater than $90 \%$ have wind speeds less than $10 \mathrm{~m} / \mathrm{s}$. For the atmosphere,, the average columnar water vapor is less than 31 $\mathrm{mm}$; and the average CLW is $0.01 \mathrm{~mm}$ and more than $97 \%$ of the scenes have CLW less than $0.1 \mathrm{~mm}$. For typical rain-free ocean scenes, there are small RTM modeling errors in the $10 \mathrm{~V}$ channel; and it is highly likely that these modeling errors are random and independent of TMI orbit position. So, although there may be small absolute offsets in the mean value of the calibration bias (due to errors in estimating the match-up environmental conditions and the RTM modeling); nevertheless, the relative change of this bias with orbit position is estimated very robustly. 




(a) Wind speed.

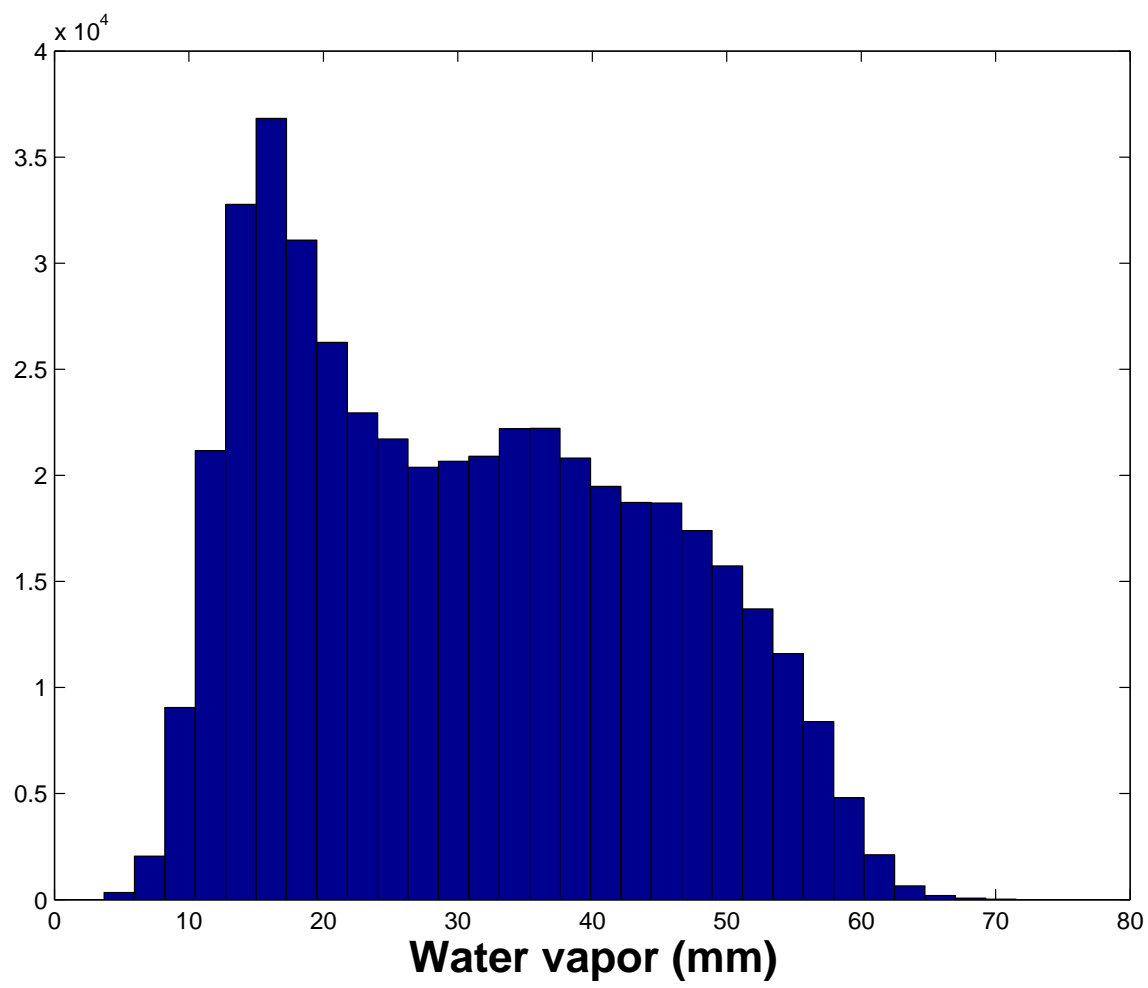

(b) Columnar water vapor.

Figure 3.8: Typical histograms of WS, WV and CLW for rain-free ocean scenes. 


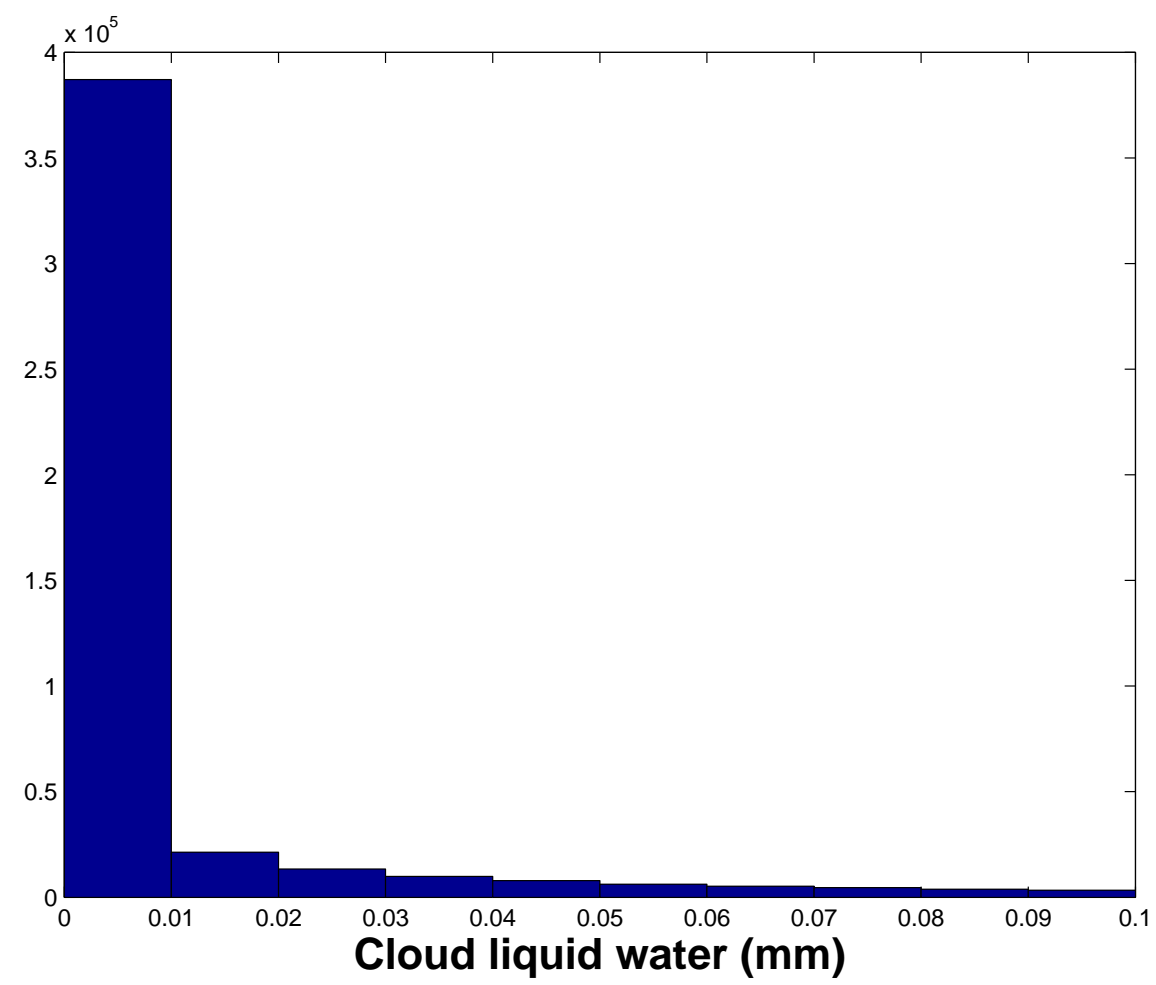

(c) Cloud liquid water.

Figure 3.8: Typical histograms of WS, WV and CLW for rain-free ocean scenes in the match-up dataset. 


\subsubsection{RTM Validation}

The Tb modeling in this dissertation was performed using the ICWG consensus RTM implementation described in Chapter 2. The RTM implementation was validated by comparing the modeled Tb's with an independent implementation of the ICWG RTM by Professor Thomas Wilheit, Department of Atmospheric Sciences of the Texas A \& M University. Both RTM implementations contained 100 layers, with each layer having a thickness of 200m. Our RTM was implemented in MATLAB and C, while the Wilheit implementation used Fortran 90. The modeled Tb's for the ensemble of 63 atmospheric profiles described previously from both implementations were compared for the TMI channels. For the comparison, both implementations used identical surface parameters of SST $=300 \mathrm{~K}$, salinity $=30 \mathrm{ppt}$ and wind speed $=10 \mathrm{~m} / \mathrm{s}$. Thus, both RTM implementations had identical structures (i.e. identical number and thickness of layers, identical emissivity and atmospheric absorption models) and identical environmental inputs. The only difference is in the software implementations, which were performed independently using different programming languages. As expected, the modeled Tb's from the two implementations were essentially identical; with differences of less than $0.001 \mathrm{~K}$ that were caused by slight differences in the precision between MATLAB and Fortran.

\subsubsection{TMI Tb Bias Determination}

The TMI bias determination involved the calculation of Tb differences between observed and theoretical RTM Tb's on a short-time scale windows ( $\sim 5 \mathrm{~min})$. The environmental parameters from GDAS are matched-up with the mean TMI Tb observations for 
each $1^{\circ}$ box and are used to calculate the predicted $\mathrm{Tb}$ for that box. One difference from the radiative transfer modeling methodology used for inter-calibration is that GDAS cloud liquid water (CLW) values are used instead of REMSS retrievals. The TMI bias correction requires modeled Tb's only for the $10 \mathrm{~V}$ channel, which is very insensitive to CLW (as discussed above); thus, the RTM modeling was simplified by using the GDAS columnar CLW values, even though they are not as well-correlated with the TMI observations as the REMSS values. To evaluate the effect of this simplification, the bias estimates were generated using both GDAS and REMSS cloud liquid values for one day, and there was negligible difference between the two cases. Figure 3.9 shows the histogram of the differences between the modeled Tb's using REMSS and GDAS CLW values for one day, which contains more than 14,000 1 degree boxes. The Tb's modeled using the REMSS values are higher by around 0.04K. Since, the histogram follows a Gaussian distribution with a near zero mean value, the use of GDAS CLW does not significantly impact the estimated bias values. This behavior is consistent across the one year time period. 


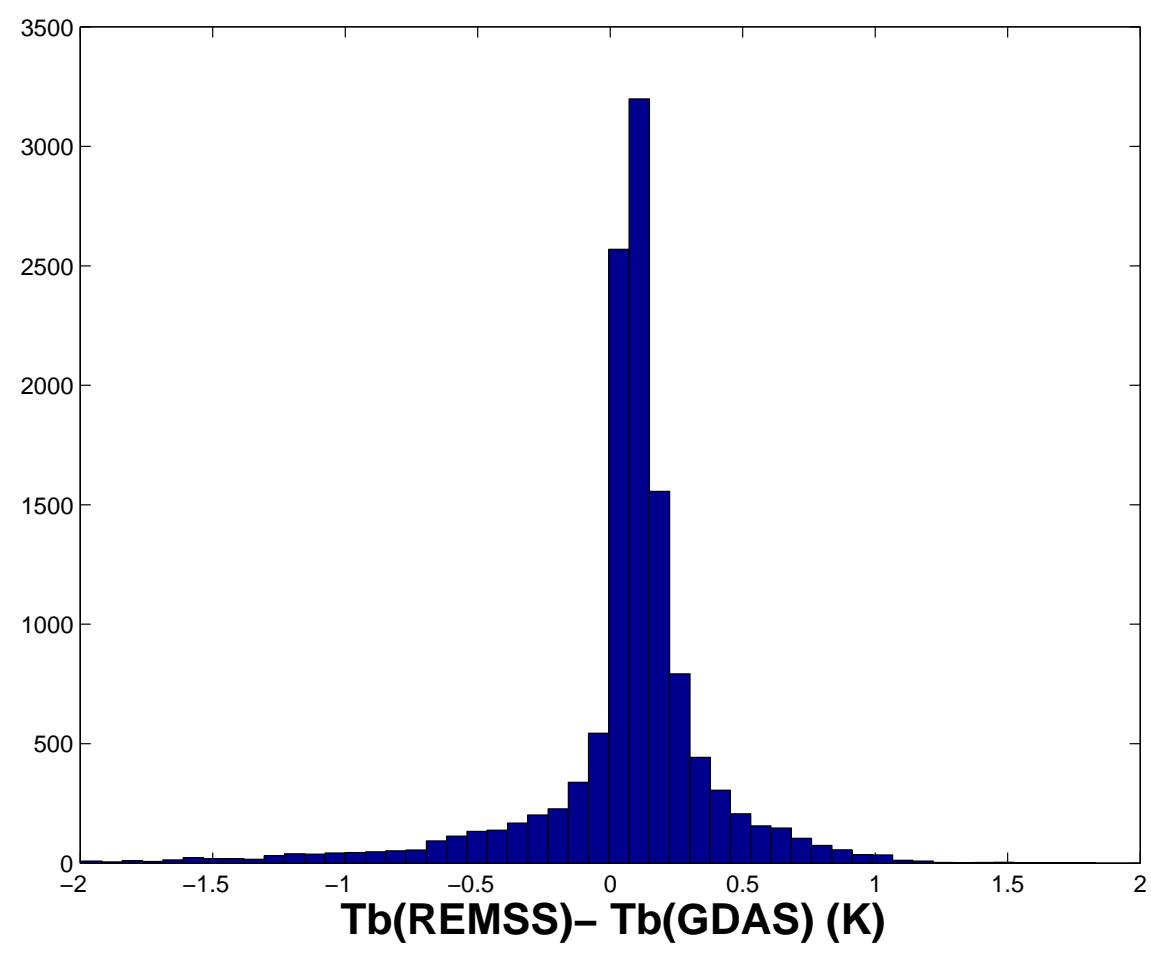

Figure 3.9: Effect of replacing REMSS CLW with GDAS CLW.

The TMI 10V Tb's for each orbit in the four-year period (2004 - 2007) are extracted from the TMI Level $1 \mathrm{C}$ data set and are averaged spatially into $1^{\circ}$ boxes, which typically have 50-100 samples. These $\mathrm{Tb}$ measurements are then filtered to remove boxes with high standard deviations and $\mathrm{Tb}$ values that are higher than upper limits expected from rain-free ocean observations, as described in Section 2.3. Figure 3.10 shows the histograms of the bias estimates for all the $1^{\circ}$ boxes on July 1, 2005 after various stages of filtering. Panel-(a). shows the histogram of the estimated biases when no filtering is applied, which has two modes: at $2 \mathrm{~K}$ and at $110 \mathrm{~K}$. The large bias estimates are caused due to high observed Tb's over land; because the RTM implementation is an ocean/atmosphere model only and does not contain any mechanisms to calculate land emissivities. Thus, land filtering is required to remove the anomalous bias estimates over land. Panel-(b). shows the histogram of the 
estimated bias after the data is filtered using oceanic upper thresholds for the observed TMI Tb's. This eliminates all the 1 degree boxes with estimated biases over 100K, and the bias estimates are now bounded within $20 \mathrm{~K}$. The mean value of the bias estimate is $1.36 \mathrm{~K}$, and the standard deviation is $1.83 \mathrm{~K}$. The bias estimates greater than $10 \mathrm{~K}$ are unrealistic, and are likely to be caused by observations containing rainy scenes. The standard deviation filter, which detects rainy pixels, is added, and the estimated bias histogram is shown in Panel-(c). The bias estimates are now in between 10K. The mean value of the bias estimate reduces to $0.75 \mathrm{~K}$, and the standard deviation is $1 \mathrm{~K}$. High CLW content is another good indicator of rainy pixels. Panel-(d). shows the effect of eliminating 1 degree boxes with CLW greater than $0.1 \mathrm{~mm}$. The outliers in the positive side remain, but the negative outliers $(<-4 \mathrm{~K})$ are removed. This is an indication that GDAS overestimates the CLW in some pixels, resulting in unrealistically high modeled Tb's. The CLW filtering does not significantly impact the mean bias value, and reduces the standard deviation to $0.9 \mathrm{~K}$. The outliers in the positive side are likely caused by "missed clouds", i.e. they are 1 degree boxes where GDAS significantly underestimates the cloud liquid water content. These outliers are few in number and their effect is greatly reduced averaging many one degree boxes to estimate the TMI time-varying bias; this procedure is described in the next section. 


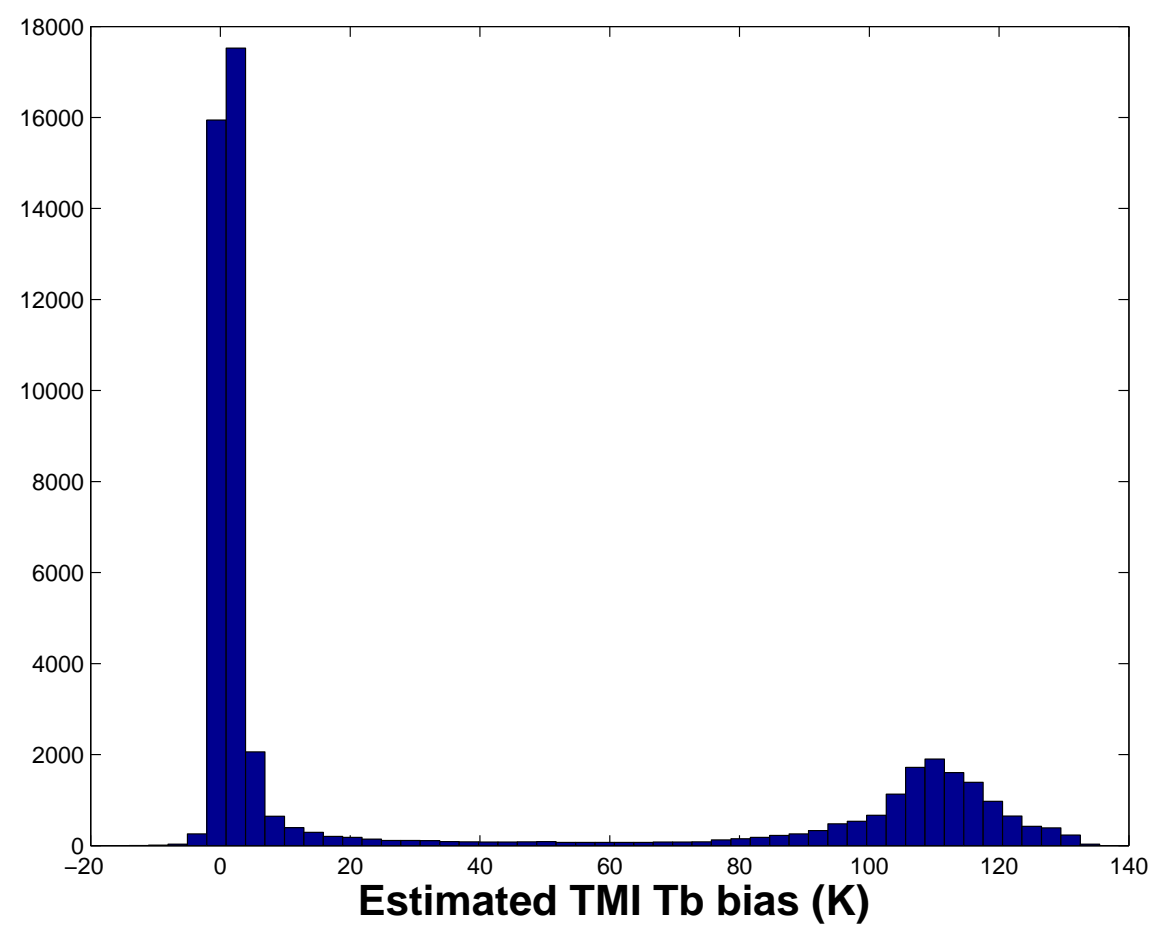

(a) No filtering.

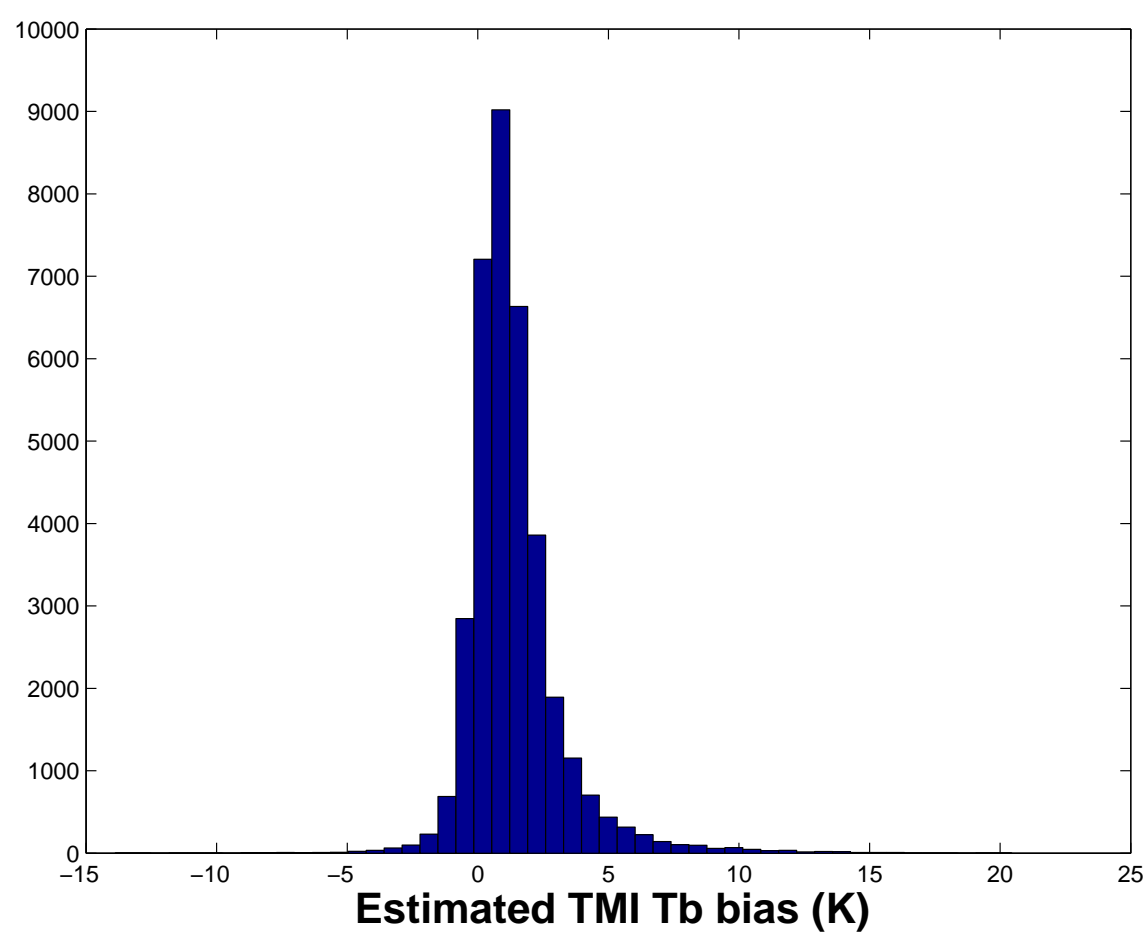

(b) Upper Tb threshold.

Figure 3.10: Results of filtering based on upper limit Tb thresholds, std. dev. thresholds and CLW. 


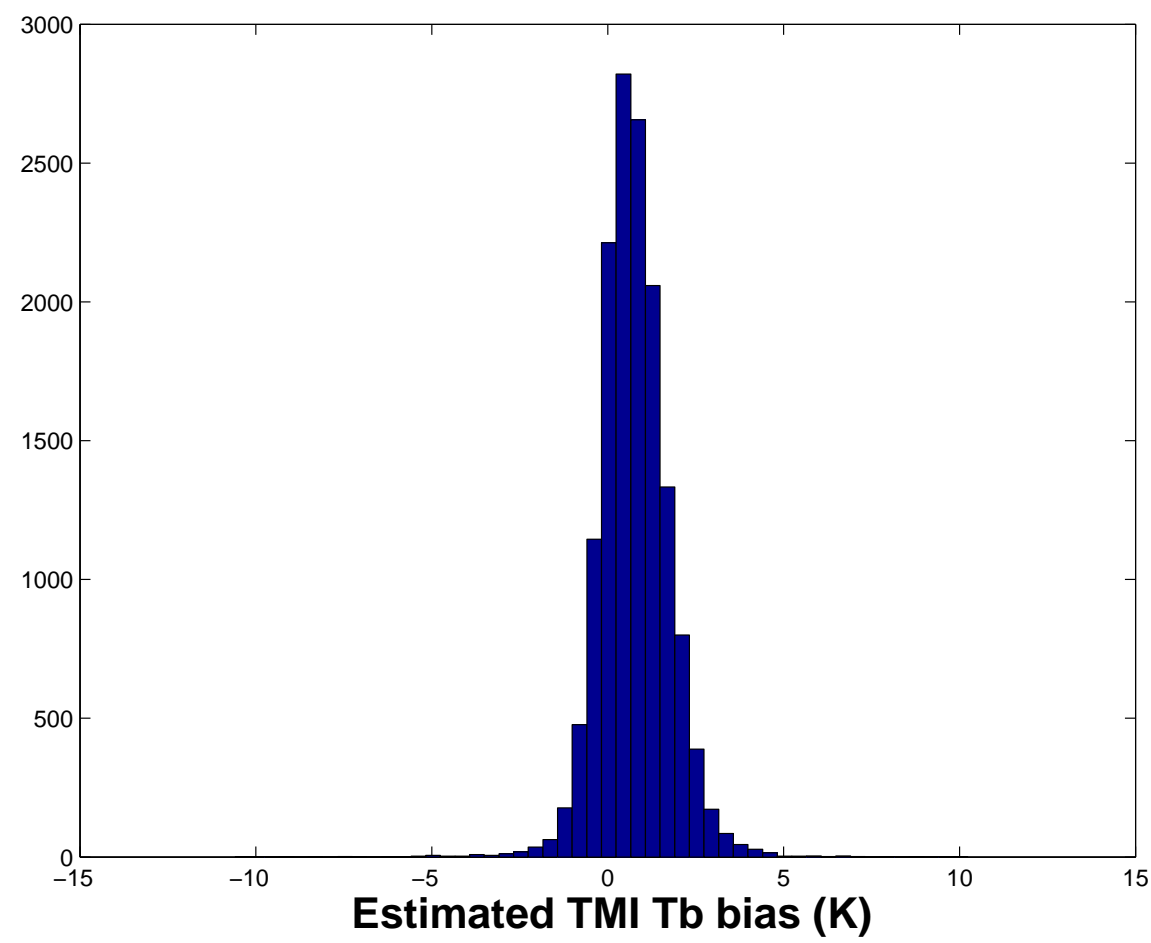

(c) Std. dev. threshold.

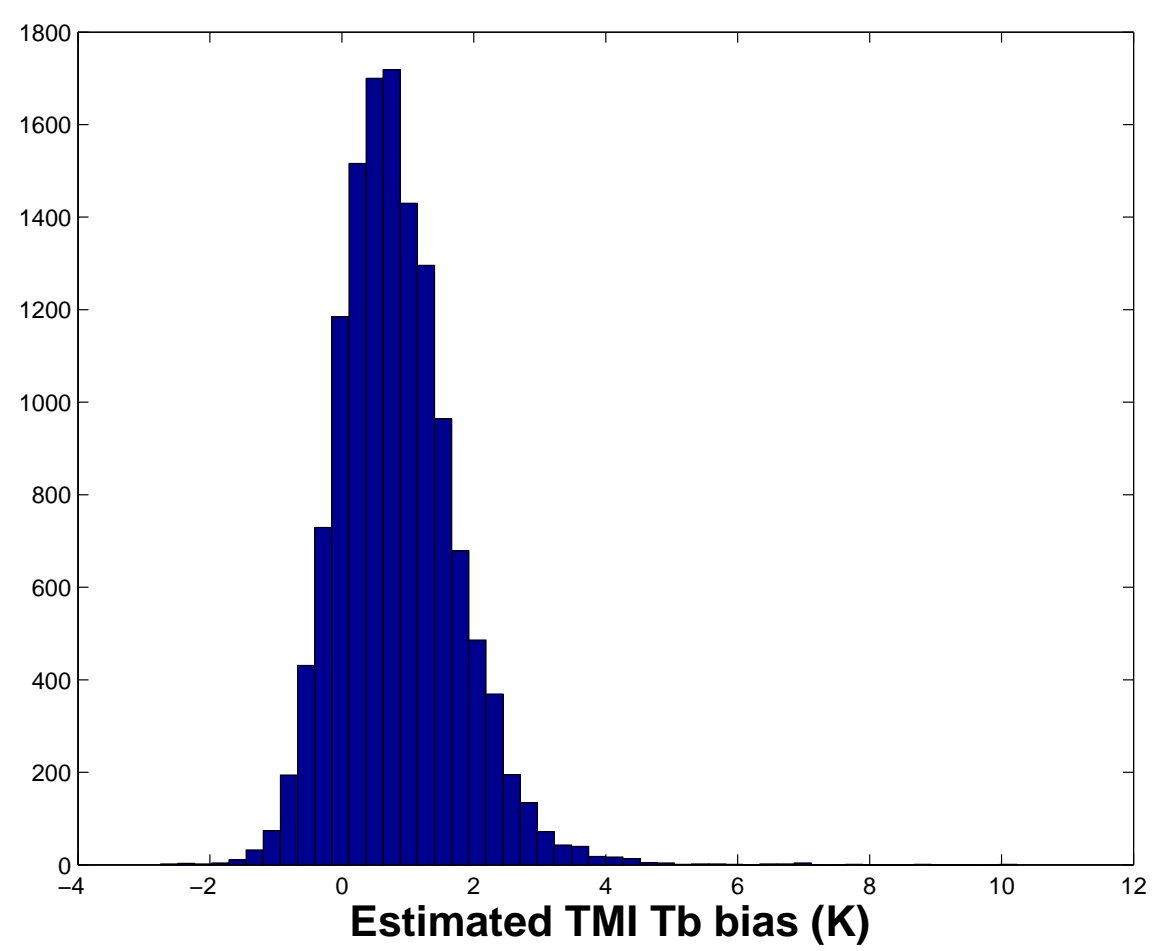

(d) CLW threshold.

Figure 3.10: Results of filtering based on upper Tb thresholds, std. dev. thresholds and CLW. 


\subsection{TMI Bias Averaging by Orbit Segments}

After quality control filtering, the average Tb bias for each box is calculated. The estimated bias for each 1 degree box, $T b_{b i a s}$, is defined as:

$$
T b_{\text {bias }}=<T b_{o b s}>-T b_{R T M}
$$

where

$<T b_{o b s}>$ is the mean observed TMI brightness temperature for the 1 degree box and

$T b_{R T M}$ is the RTM modeled $\mathrm{Tb}$ using the environmental parameter match-ups for that box.

The TMI ground swath for each orbit is divided into 5-minute segments, as shown in Fig. 3.11, where each segment is shaded with a different gray scale. The orbital time $t_{o}$ of each box is defined as the elapsed time since the start of the ascending pass, which corresponds to the beginning of the Level $1 \mathrm{C}$ file. The calculated biases, from all the orbits in a single day that lie within a given 5-minute orbit time segment, are combined to form a daily average orbit. Thus, the estimated bias for each 5 minute segment is :

$$
T b_{\text {est }}(i)=\frac{\sum_{t_{o} \in T_{i}} T b_{\text {bias }}\left(t_{o}\right)}{n_{i}}
$$

where : 
$T b_{\text {est }}$ is the estimated bias for each five-minute segment for a given day

$i$ denotes the orbit segment index which extends from 0 to 18 , i.e. 0 minutes to 90 minutes

$T_{i}$ is the orbit time range of the corresponding 5 minute segment (for example, $T_{1}=5$ min corresponds to the time window: $\left.2.5-7.5 \mathrm{~min}\right)$

$T b_{\text {bias }}$ is the estimated bias for each 1 degree box and to is the orbit time for each bias

$n_{i}$ is the number of 1 degree boxes in the ith 5 minute segment

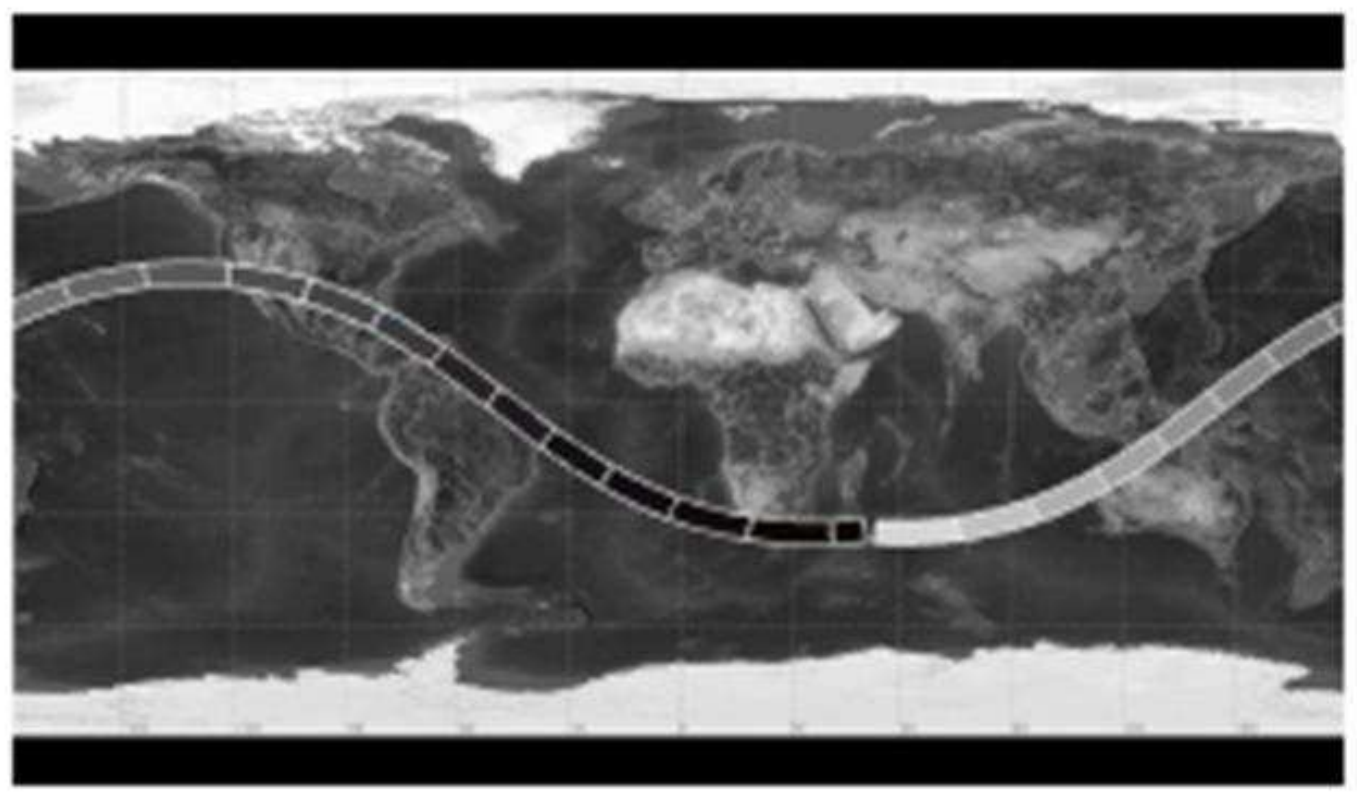

Figure 3.11: TMI ground swath for one orbit with markers at five-minute intervals. 
For each five-minute orbit segment, the $\mathrm{Tb}$ bias estimates are the average of about 200 to 8001 degree boxes for that orbit segment. The standard deviations of the $\mathrm{Tb}$ bias estimate for all these five-minute orbit segments for one month were observed and were found to be essentially constant through the orbit. The results given in Fig. 3.12 show that the average standard deviation is $\sim 0.5 \mathrm{~K}$ through the orbit.

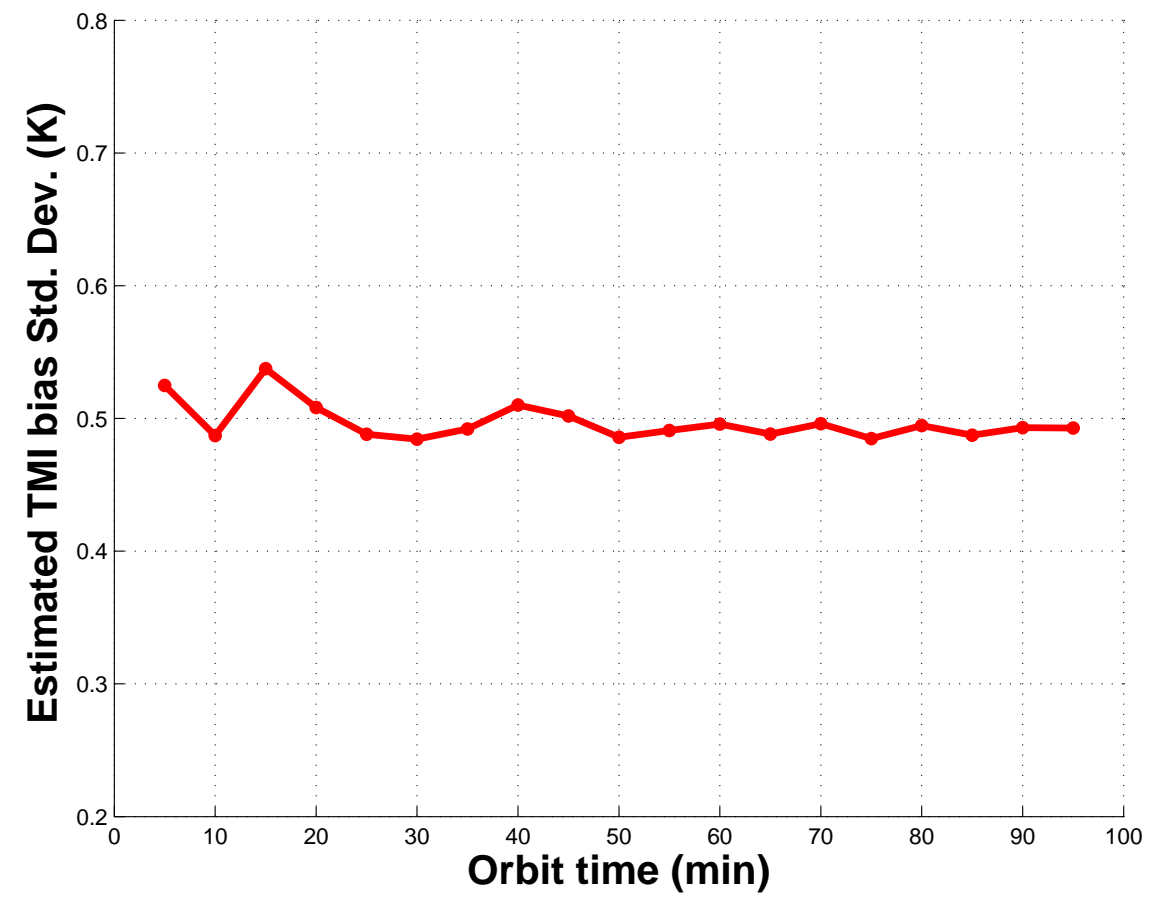

Figure 3.12: Standard deviation of the estimate of the Tb bias for a one-month average orbit. 
An analysis of the dependence of the TMI $10 \mathrm{~V}$ bias on the orbit time was performed to get an understanding of the heating and cooling patterns of the reflector within a TRMM orbit. Figure 3.13 shows the daily average orbit bias from two different seasons for June 10, 2006 and October 23, 2005, which are produced by averaging the corresponding 5-minute segments for all of the 15 orbits of that day. The shaded area shows when the TRMM satellite is in darkness (eclipse), and for clarity of presentation, the x-axis is relative time beginning with the umbra entry. These two daily Tb bias patterns have significant differences; yet there are many similarities.

Concerning the similarities, when entering into eclipse, there is a rapid drop in the observation bias during darkness $(>1.5 \mathrm{~K})$, which coincides with the expected cooling of the reflector, and after TMI is exposed to sunlight, the Tb bias increase immediately, which coincides with the expected rise in the reflector physical temperature. This general behavior indicates that the radiometric bias is proportional to the reflector temperature; however because of the differences in the shapes and level offset patterns it appears that the biases must be determined for each day. For example, there are seasonal differences, where the Tb bias during sun-light is $+0.75 \mathrm{~K}$ on June 10 th and $+1.5 \mathrm{~K}$ on October $23 \mathrm{rd}$; and the corresponding minimum $\mathrm{Tb}$ bias was $-0.5 \mathrm{~K}$ and $-1.0 \mathrm{~K}$ respectively. The seasonal differences can be associated with the different solar beta angles, which will be discussed further during Chapter-4. The total range of the bias variation is $\sim 2.5 \mathrm{~K}$, which is similar to the results reported in the initial TMI on-orbit evaluation by Wentz et al. [7]. 
TMI Bias-Oct 23, 2005 [ $\left.\beta=-38.56^{\circ}\right]$



(a) October 23, 2005

TMl Bias- Jun 10, $2006\left[\beta=+15.03^{\circ}\right]$



(b) June 10, 2006

Figure 3.13: Orbital pattern for TMI 10V bias averaged over one day for two different seasons. 
An estimate of the reflector physical temperature variation (about the mean) over an orbit associated with the daily bias variation can be derived by dividing the peak to peak $\mathrm{Tb}$ bias by the estimated emissivity of the reflector. The daily estimated peak-to-peak variation of the reflector physical temperature for the ICWG one-year period is shown in Fig. 3.14. This physical temperature variation reaches a maximum of $70 \mathrm{~K}$; which is consistent with other independent estimates of the orbital variation of the TMI reflector physical temperature $[7,34]$. Further, the peak to peak extent and the shape of the Tb bias orbital variation vary significantly from day to day.

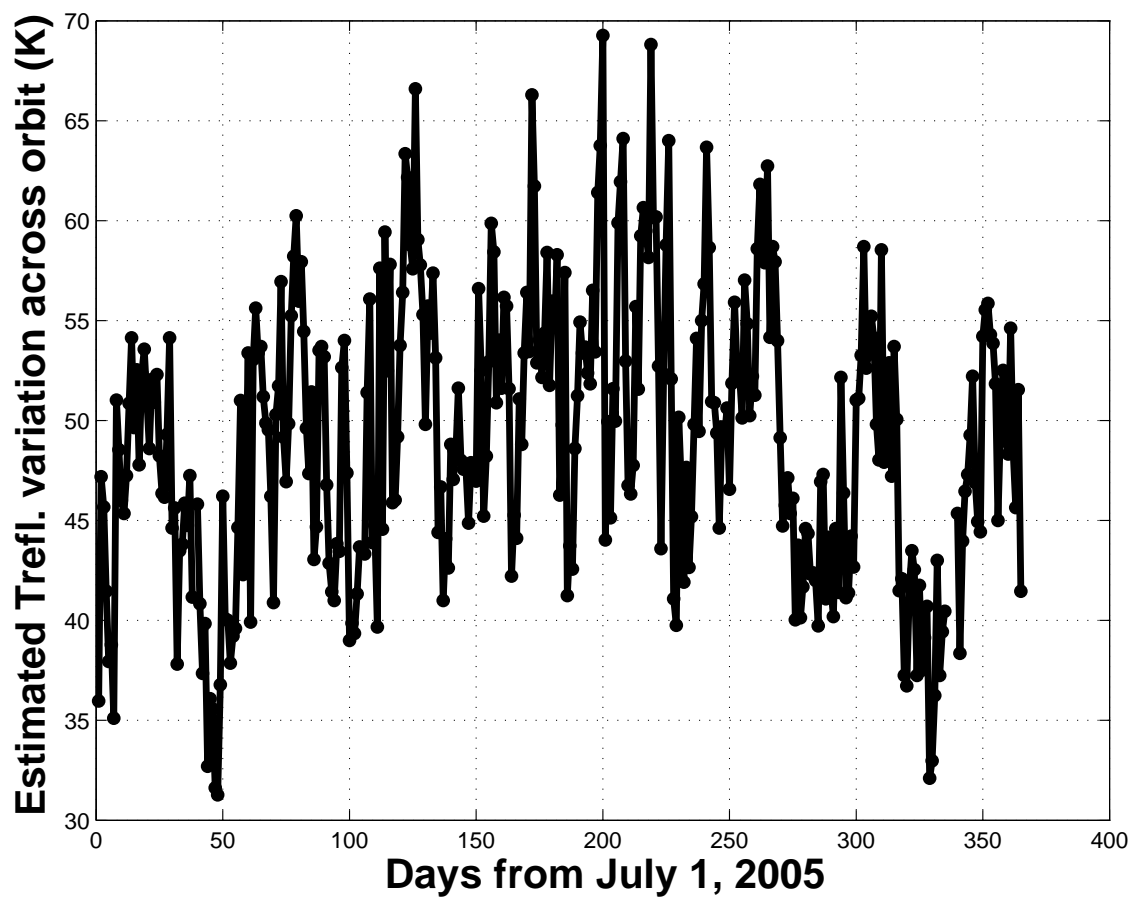

Figure 3.14: Estimated TMI reflector temperature variation over an orbit. 
To understand this phenomena, the daily bias orbital patterns were analyzed for the TRMM orbit precession time period of $\sim 47$ days. We discovered a correlation between the magnitude of the peak to peak $\mathrm{Tb}$ bias variation and the duration of the eclipse period as shown in Fig. 3.15. Over this 47 day period, the envelope of the peak to peak $\mathrm{Tb}$ bias varies from about $2 \mathrm{~K}$ to $1.2 \mathrm{~K}$; and the corresponding duration of the TRMM eclipse period varies from 25 to 36 minutes. Since the cooling of the reflector is governed by the length of the eclipse period, this observation seems consistent with the hypothesis that the $\mathrm{Tb}$ bias is produced by the reflector temperature change; however there is an unexplained high frequency variability in the $\mathrm{Tb}$ bias pattern. 


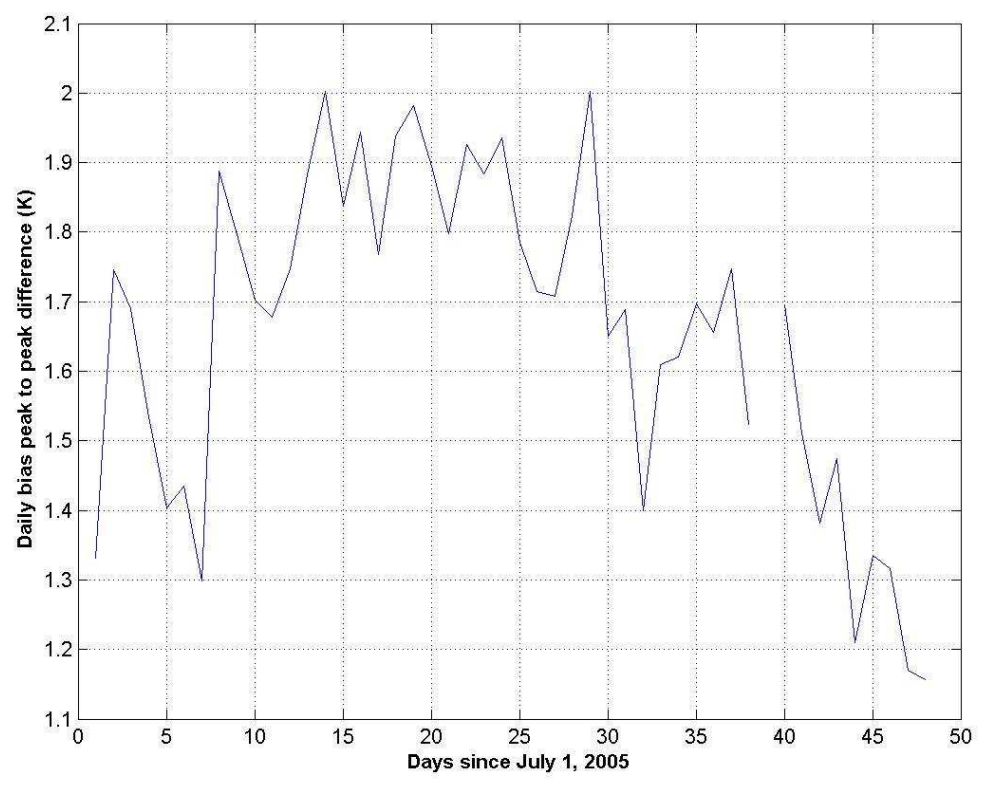

(a) Peak to peak difference

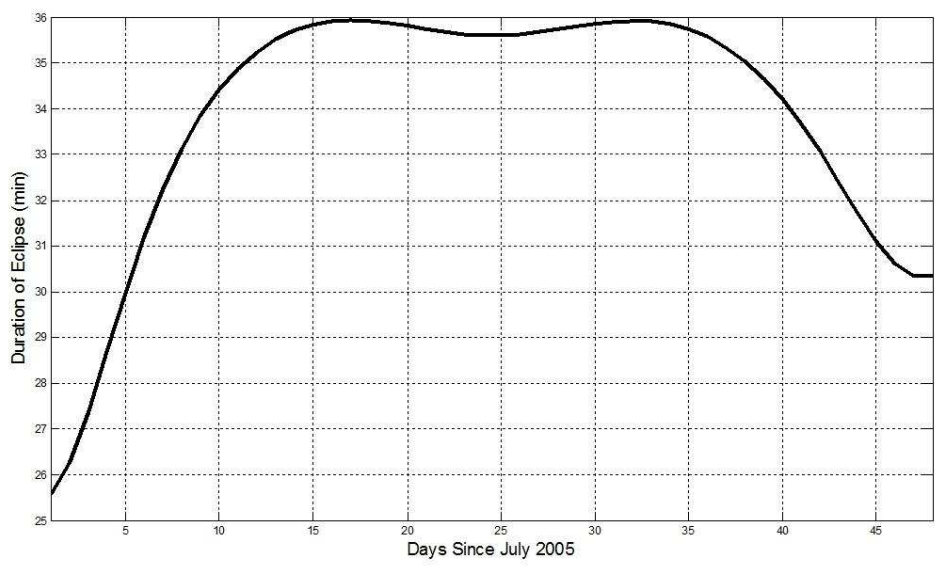

(b) Duration of eclipse

Figure 3.15: Daily TMI bias characteristics (July 1, 2005 - August 17, 2005) 
A more careful examination of the orbital $\mathrm{Tb}$ bias patterns was conducted on a dayby-day basis for the entire one-year of the ICWG period. This revealed several perplexing $\mathrm{Tb}$ bias patterns that could not be explained by the simplistic notion that heating occurred during the sun-lighted portion of the TRMM orbit and cooling resulted during the eclipse portion. For example, Fig. 3.16 panel-(a) shows the orbital bias plot for July 18, 2005, where there is a period of approximately 32 minutes after TRMM exits the earth's shadow, where the bias remains constant (cold). The fact that the $\mathrm{Tb}$ bias does not increase, implies that the reflector temperature does not increase. To understand how this might occur, we repeat Fig. 1.1 here to show that the location of the TMI on the top of the TRMM spacecraft could cause it to be shadowed from sunlight if the sun were to rise behind the satellite buss structure ("dog house").

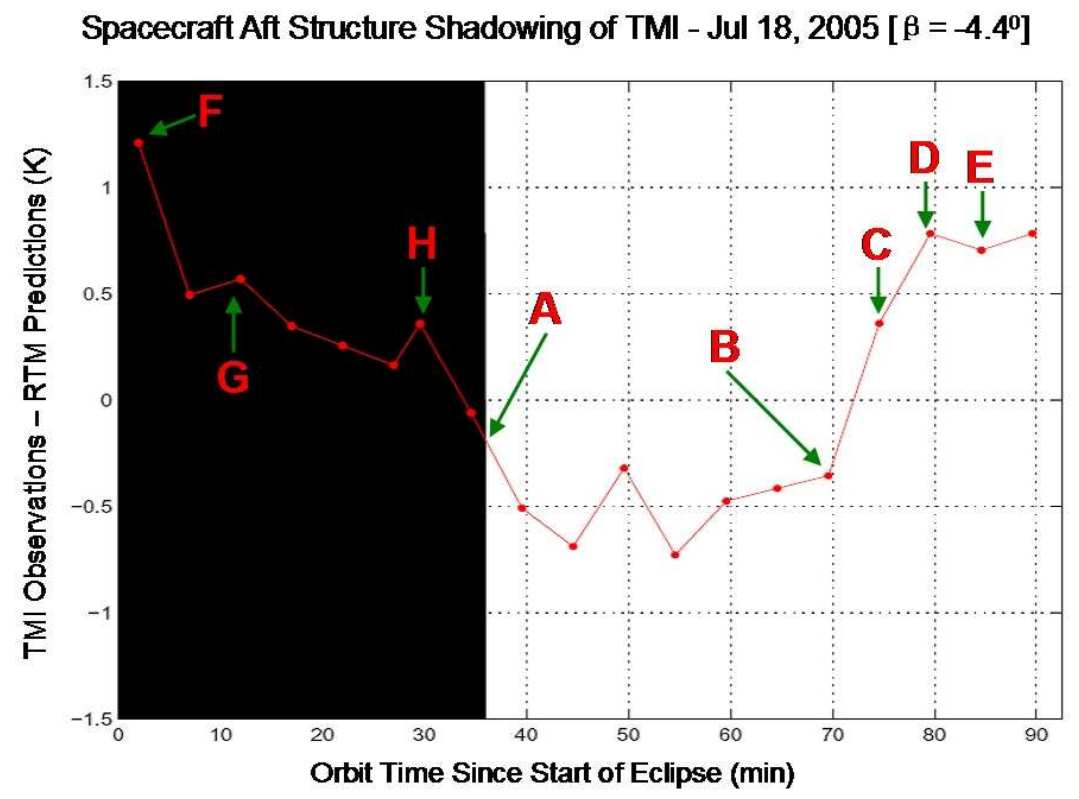

(a) Estimated bias variation with orbit time.

Figure 3.16: STK analysis of TRMM spacecraft shadowing for July 18, 2005. 


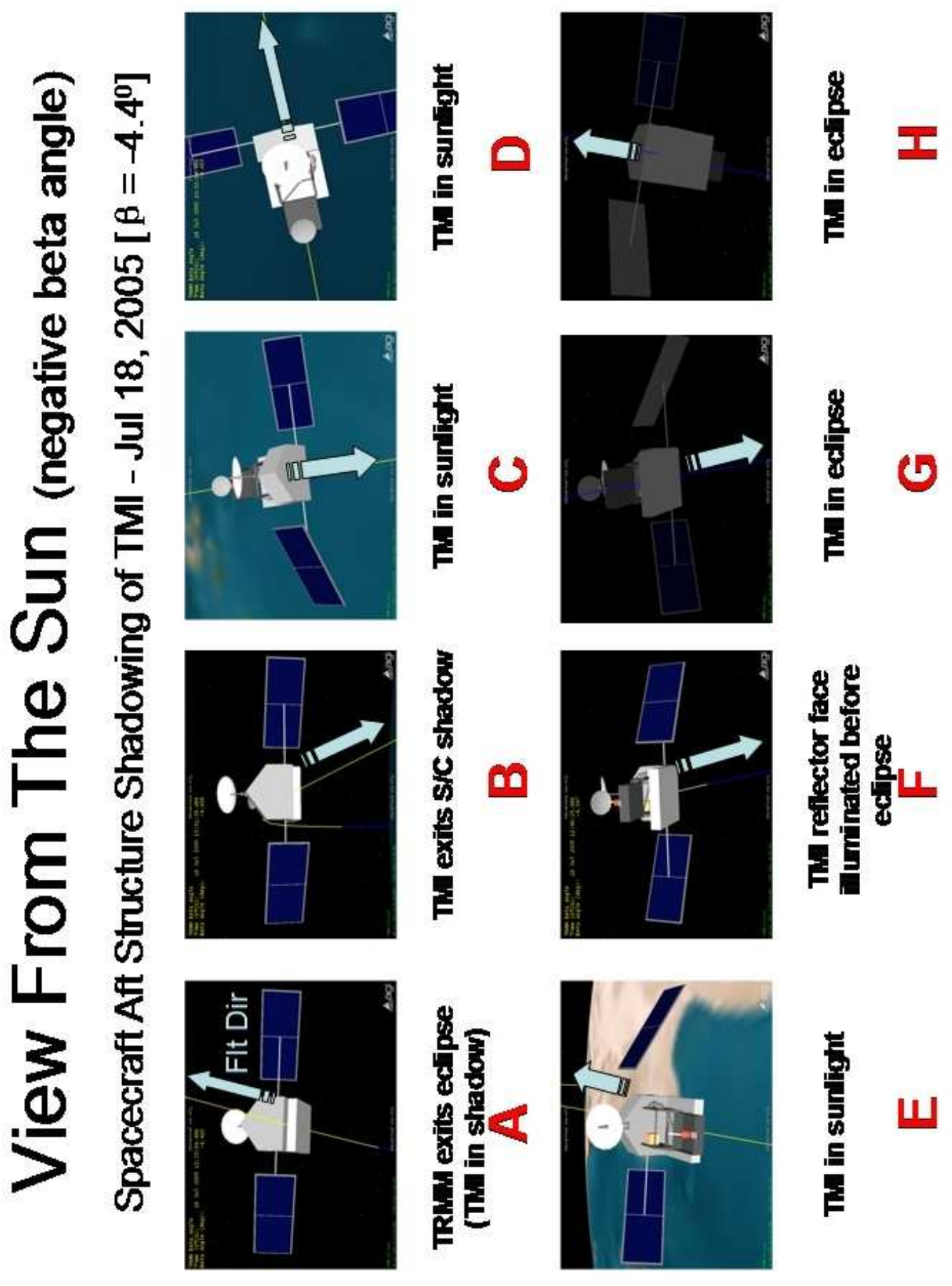

(b) View of TRMM from the sun.

Figure 3.16: STK analysis of TRMM spacecraft shadowing for July 18, 2005. 


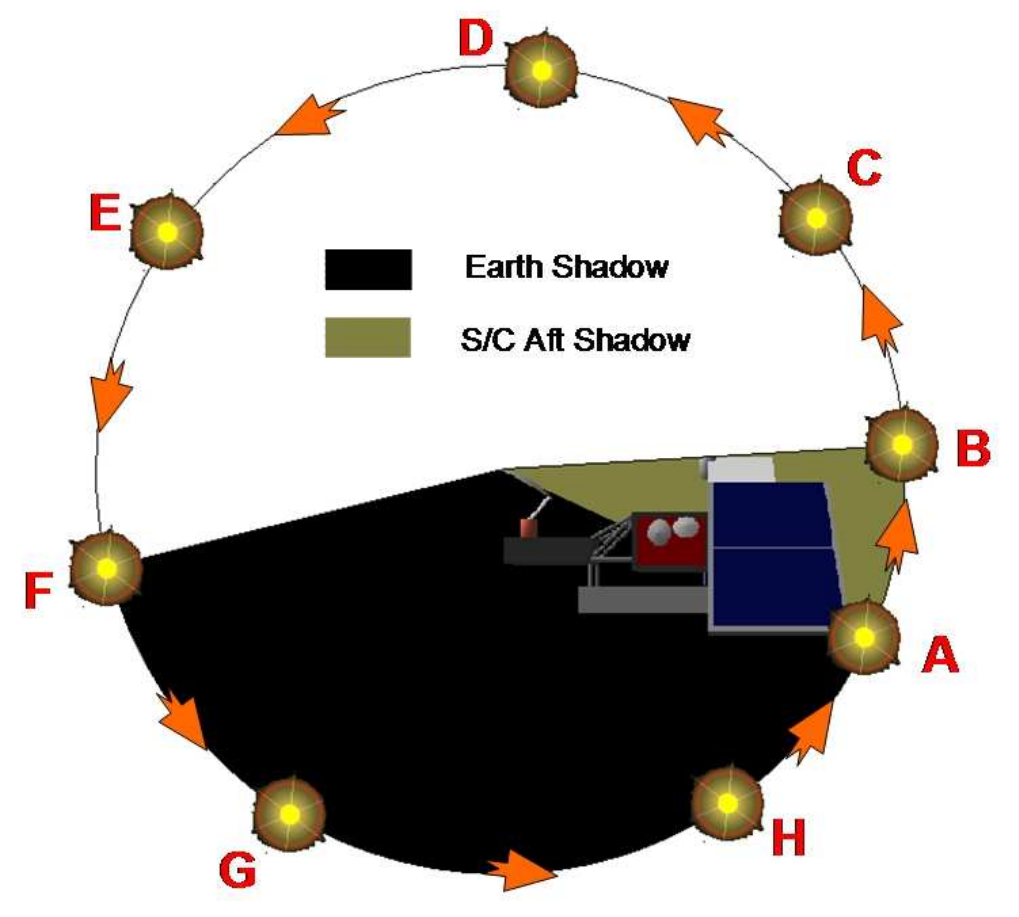

(c) Schematic of earth and spacecraft shadowing effects.

Figure 3.16: STK analysis of TRMM spacecraft shadowing for July 18, 2005.

After performing a 3-D visualization of the sun vector illumination of TRMM using Satellite Tool Kit [35], it was determined that this behavior is caused by the shadow cast by the TRMM spacecraft structure, which shields the TMI reflector from solar heating. A series of frames from a STK animation of the TRMM spacecraft are shown in Fig. 3.16(b) [36], where each sub-panel -A through -H represents a time sequence of satellite images as viewed from the sun. Where the sun illuminates a portion of the satellite, the image is shaded a bright-white color; and where the satellite is not illuminated, the shading is a darker shade of grey. For example in sub-panels-A through -F, the TRMM is illuminated by sunlight; but in sub-panels- $\mathrm{G}$ and $-\mathrm{H}$ the satellite in the earth shadow (eclipse darkness). Also, whenever the TMI is visible in the image, then it will be intercepting solar flux, which 


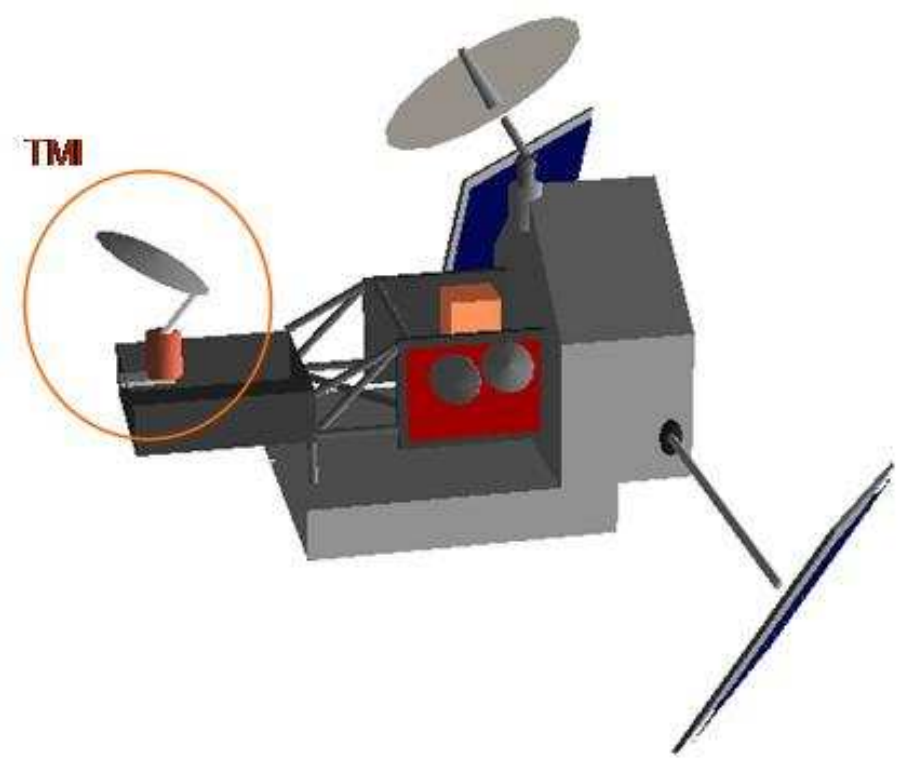

Fig. 1.1 (repeated) : The TRMM observatory.

will warm the spinning reflector. The time series of Tb biases (Fig. 3.16-(a)) is related to the sun/TRMM geometry in Fig. 3.16-(b) by the labels -A through -H; and an alternate representation is presented in Fig. 3.16-(c) where the sun illumination geometry is presented in a polar diagram [36] with the associated "call-outs" to the STK frames -A through -H. For example, in Fig. 3.16-(c), as time progresses, the sun rises and begins illuminating the rear of the spinning TMI reflector between STK frames-B and -C; and the bias (reflector physical temperature) monotonically increases until STK frame-D, where it plateaus until frame-F. Afterwards, the TRMM passes into eclipse between frames-F and -G and the cycle repeats. 
There are other days where the bias value drops sharply before TRMM enters the earth's shadow; and this was also determined to be an effect of spacecraft shadowing. Figure 3.17 [36] illustrates the effects of spacecraft shadowing on the bias patterns for August 2, 2005; and Fig. 3.17b, sub-panel-A, depicts the time when TRMM exits darkness and the sun illuminates the face of the reflector. The bias monotonically increases until sub-panel-D where it plateaus. After sub-panel-E the reflector enters into the spacecraft shadow and the bias sharply decreases even though the TRMM spacecraft is in sunlight. The spacecraft then enters darkness (sub-panel-G), after which the bias continues to drop till the end of the eclipse period.

Spacecraft Aft Structure Shadowing of TMl - Aug 02, $2005\left[\beta=+4.3^{0}\right]$

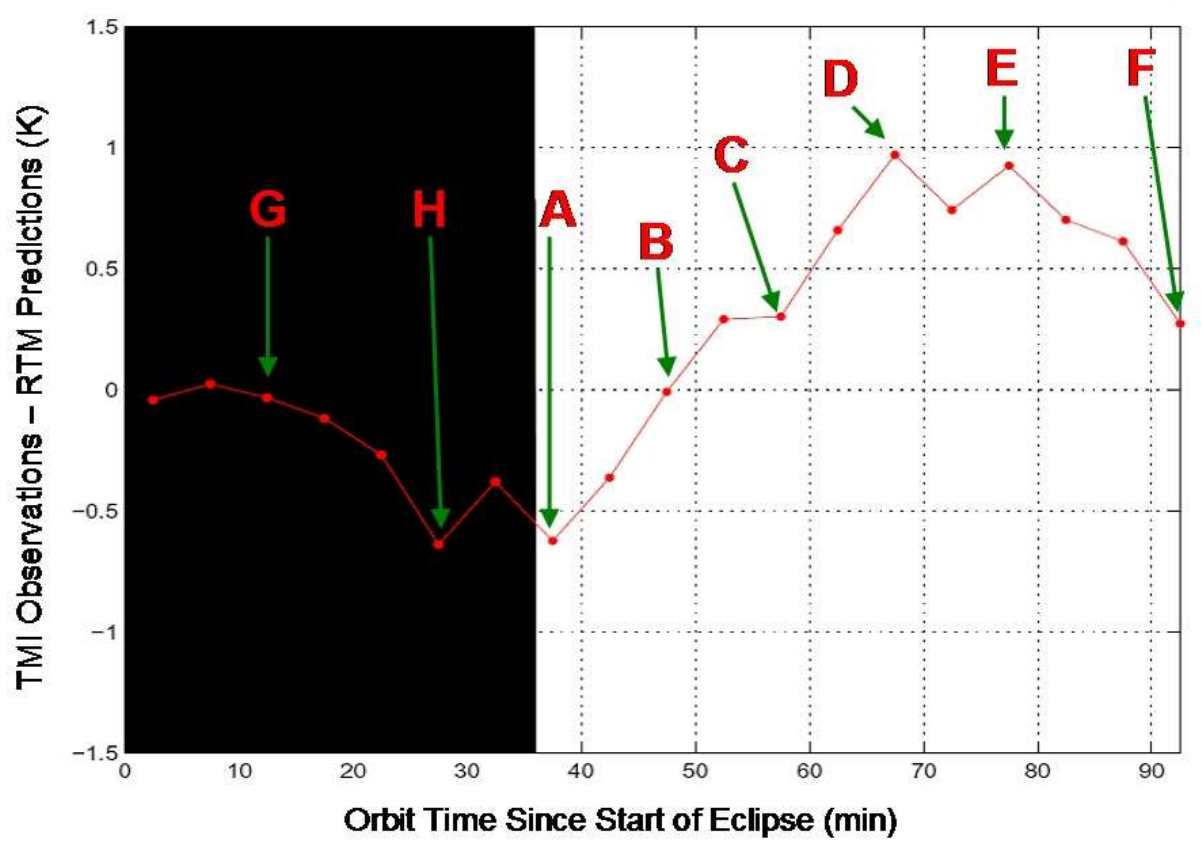

(a) Estimated bias variation with orbit time.

Figure 3.17: STK analysis of TRMM spacecraft shadowing for August 2, 2005. 


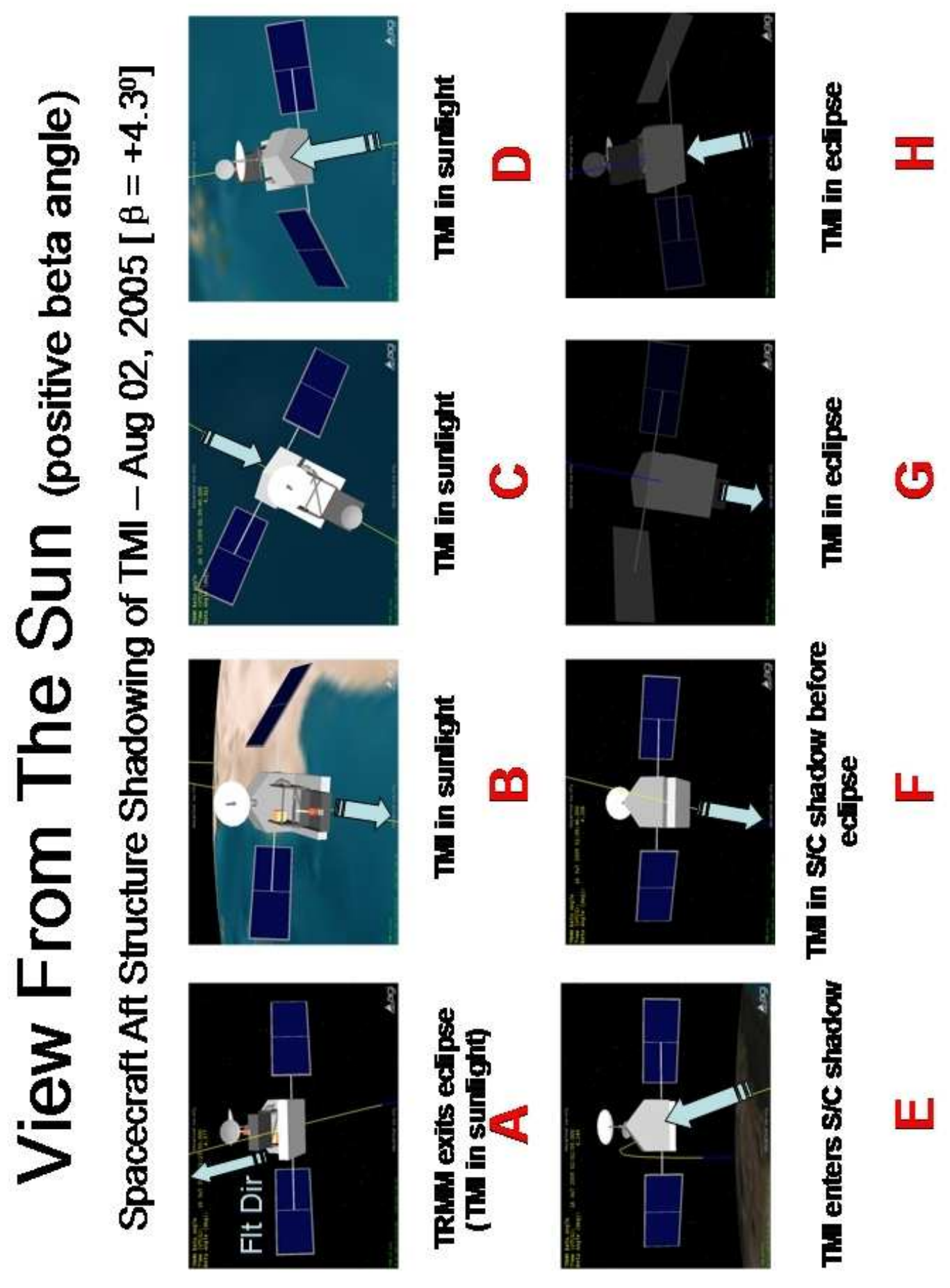

(b) View of TRMM from the sun.

Figure 3.17: STK analysis of TRMM spacecraft shadowing for August 2, 2005. 


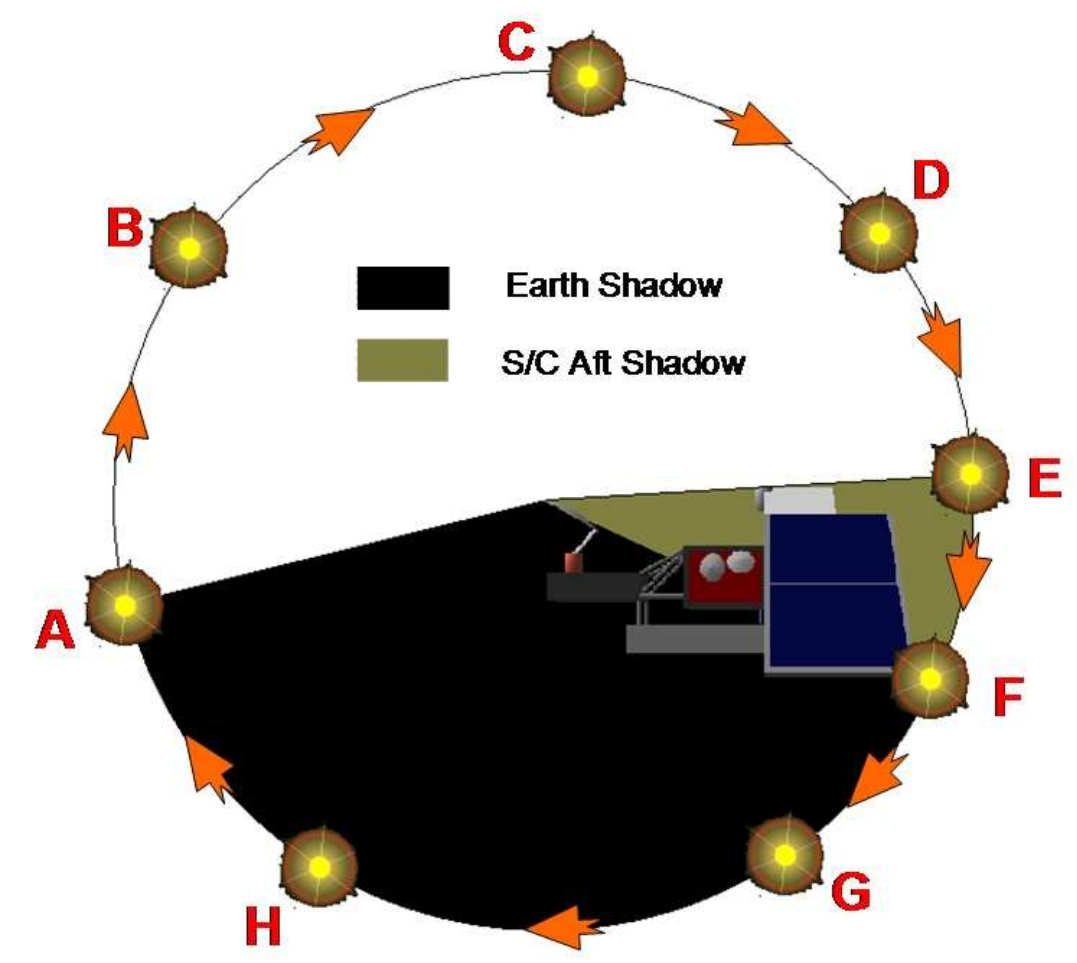

(c) Schematic of earth and spacecraft shadowing effects.

Figure 3.17: STK analysis of TRMM spacecraft shadowing for August 2, 2005.

Along with the time-variable $\mathrm{Tb}$ bias around each orbit, there is also a systematic change in the mean daily biases during 2004 and 2005, as shown in Fig. 3.18. Overall, the bias follows a pattern that is the product of the 47 day and annual cycles; however, not all of the 47 day observations follow this pattern. For instance, there are many instances where the bias appears to be rising as part of a 47 day cycle and then drops abruptly, thereby breaking the periodicity of the mean bias oscillation. Thus, the mean value of the daily bias pattern is not deterministic as a function of these two cycles. Figure 3.19 is a plot of the magnitude of the ac component of the orbital pattern of $\mathrm{Tb}$ biases (i.e., daily peak-topeak bias differences) for the four year period. This general shape of this plot appears to 
the superposition of these cycles rather than the product. But as with the mean biases, it cannot be adequately represented using a periodic function.



Figure 3.18: Mean daily bias variation for 2004 and 2005.



Figure 3.19: Daily peak-to-peak bias variation for 2004 and 2005. 
Thus, the estimated TMI bias cannot be easily modeled solely as a function of orbit time. The peak to peak magnitude of the bias oscillation varies significantly from day to day; and this variation is not periodic over the time period that was observed. Moreover, the shape of the bias oscillation over an orbit changes from day to day due to differences in the duration of the eclipse. Finally, the Tb bias variation is affected by the shadowing of the TMI reflector by the TRMM spacecraft; and such shadowing effects occur on some days but do not occur on others. As a result, the TRMM bias correction cannot be modeled solely as a function of the orbit time.

Since the bias patterns for the entire ICWG time period could not be modeled as a single function of orbit time, separate bias estimates were calculated for every day of the year and stored in a look-up table. The TMI radiometric calibration error caused by the varying reflector temperature is effectively removed by subtracting the empirically-derived daily biases from the TMI Tb measurements at all frequencies and polarizations. To verify the effectiveness of this approach, corrected TMI Tb's are compared with near-simultaneous WindSat and SSMI observations, and the results of this inter-comparison are presented next.

\subsection{Inter-comparison of Corrected TMI Tb's with WindSat and}

\section{SSMI}

The estimated correction, described in the previous section, was applied to each TMI $1^{\circ}$ box for the ICWG time period as follows : 


$$
\begin{aligned}
T b_{\text {est }}(p o l) & =\text { Look }-u p\left(\text { doy }, t_{o}\right) \\
T b_{\text {corr }}(f, p o l) & =T b_{\text {orig }}(f, p o l)-T b_{\text {est }}(p o l)
\end{aligned}
$$

where :

$T b_{e s t}$ is the estimated TMI bias

pol refers to the channel polarization

Look-up denotes the look-up table containing the estimated biases

doy is the number of days from July 1, 2005

$t_{o}$ is the orbit time in minutes

$T b_{\text {corr }}$ is the corrected TMI Tb

$f$ is the channel frequency

$T b_{\text {orig }}$ is the original TMI Tb, obtained from the Level $1 \mathrm{C}$ data product

Thus, an identical bias correction is applied to all the channels of a given polarization, regardless of the frequency. The estimated correction differs for the two polarizations because of the difference in reflector emissivity estimated by Wentz [7].

The inter-calibration procedure described in Section 2.5 was repeated using the corrected TMI Tb's. In order to verify that the TMI time-dependent bias is spectrally invariant, all the TMI channels are compared with the corresponding WindSat and SSMI channels after implementing the recommended correction that was derived from the $10 \mathrm{~V}$ channel. Table 
3.2 shows the mean values of these relative biases for the ICWG time period. Figure 3.20 shows the V-pol radiometric biases between TMI and WindSat for the corrected TMI Tb's, separated by time of day. After correction, the mean time-of-day dependence of the relative TMI/WindSat calibration reduces to less than $0.1 \mathrm{~K}$ for the $10 \mathrm{~V}$ channel, and less than $0.15 \mathrm{~K}$ in the $19 \mathrm{~V}$ and $37 \mathrm{~V}$ channels. The $21 \mathrm{~V}$ channel, which is the most sensitive to small changes in atmospheric conditions between evening and morning, has residual differences of $\sim 0.25 \mathrm{~K}$. Similar results are obtained for the relative biases between TMI and SSMI F-13 and SSMI F-14, as shown in Figures 3.21 and 3.22 respectively. This is a strong indication that the empirically derived correction effectively cancels the variable TMI radiometric calibration bias. Similar results are observed for the horizontal polarizations at all frequencies, as shown in Appendix D.

Table 3.2: Relative bias for corrected TMI Tb's

\begin{tabular}{|c|c|c|c|c|c|c|}
\hline \multirow{2}{*}{ Channel } & \multicolumn{2}{|c|}{ TMI - F13 } & \multicolumn{2}{c|}{ TMI - F14 } & \multicolumn{2}{c|}{ TMI - WindSat } \\
\cline { 2 - 7 } & Evening & Morning & Evening & Morning & Evening & Morning \\
\hline $10 \mathrm{~V}$ & - & - & - & - & 0.00 & 0.01 \\
\hline $19 \mathrm{~V}$ & -1.50 & -1.58 & -1.12 & -1.13 & -0.71 & -0.85 \\
\hline $21 \mathrm{~V}$ & -2.67 & -2.71 & -2.28 & -2.32 & -1.82 & -2.05 \\
\hline $37 \mathrm{~V}$ & -1.50 & -1.54 & -1.73 & -1.76 & -3.40 & -3.49 \\
\hline
\end{tabular}






(a) $10 \mathrm{~V}$



(b) $19 \mathrm{~V}$

Figure 3.20: Relative radiometric biases with WindSat for corrected TMI Tb's. 


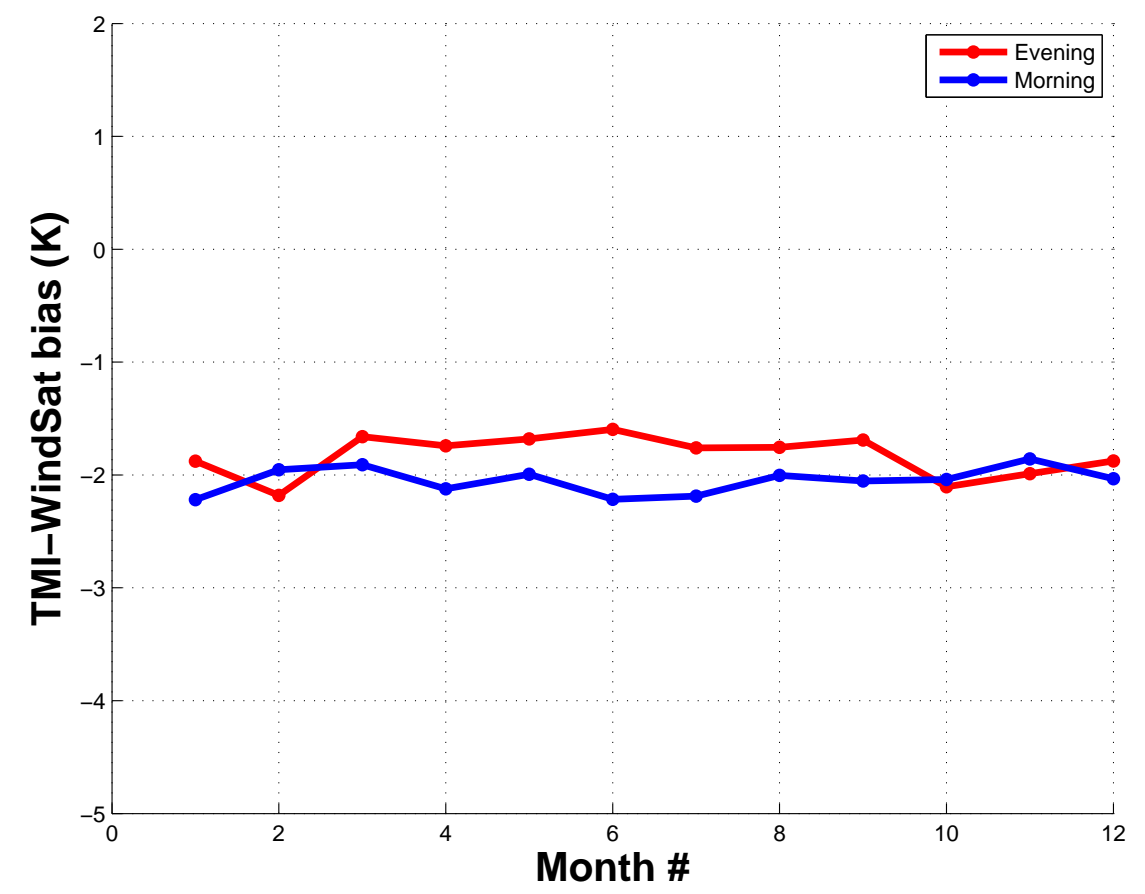

(c) $21 \mathrm{~V}$

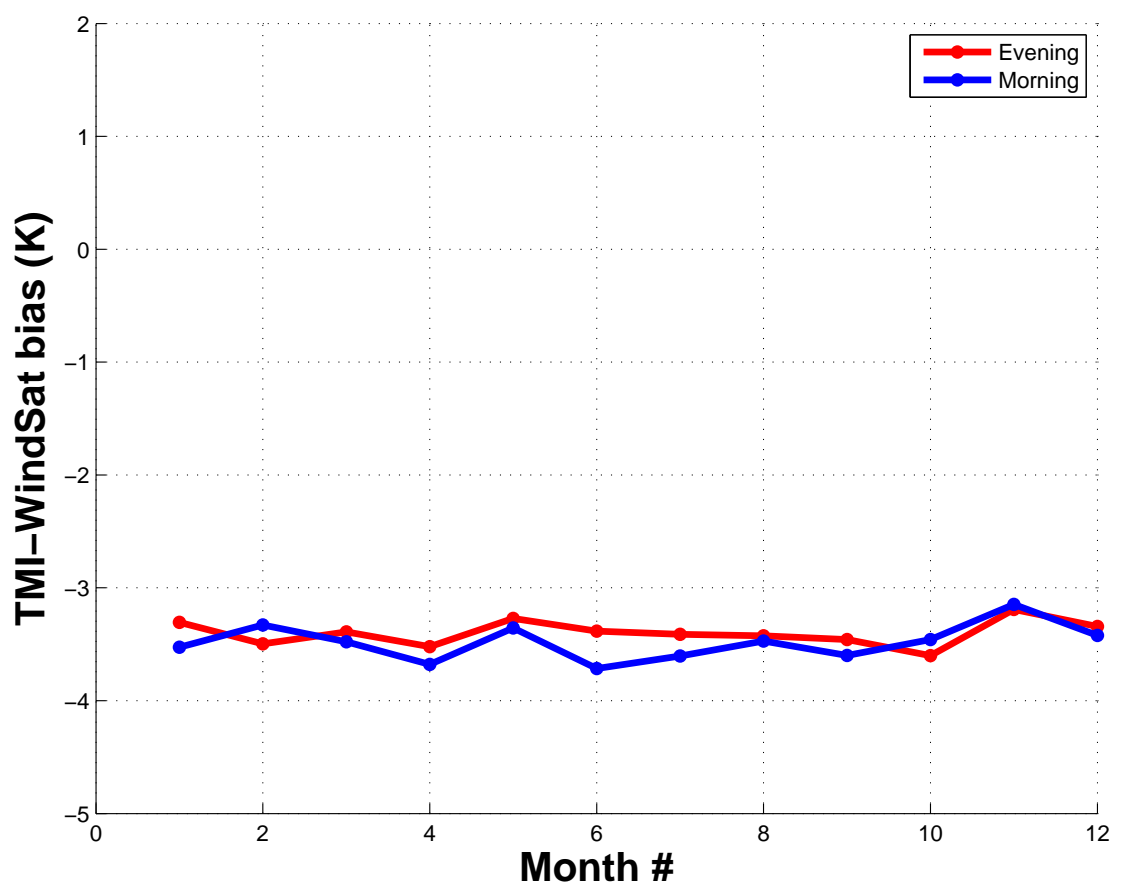

(d) $37 \mathrm{~V}$

Figure 3.20: Relative radiometric biases with WindSat for corrected TMI Tb's. 


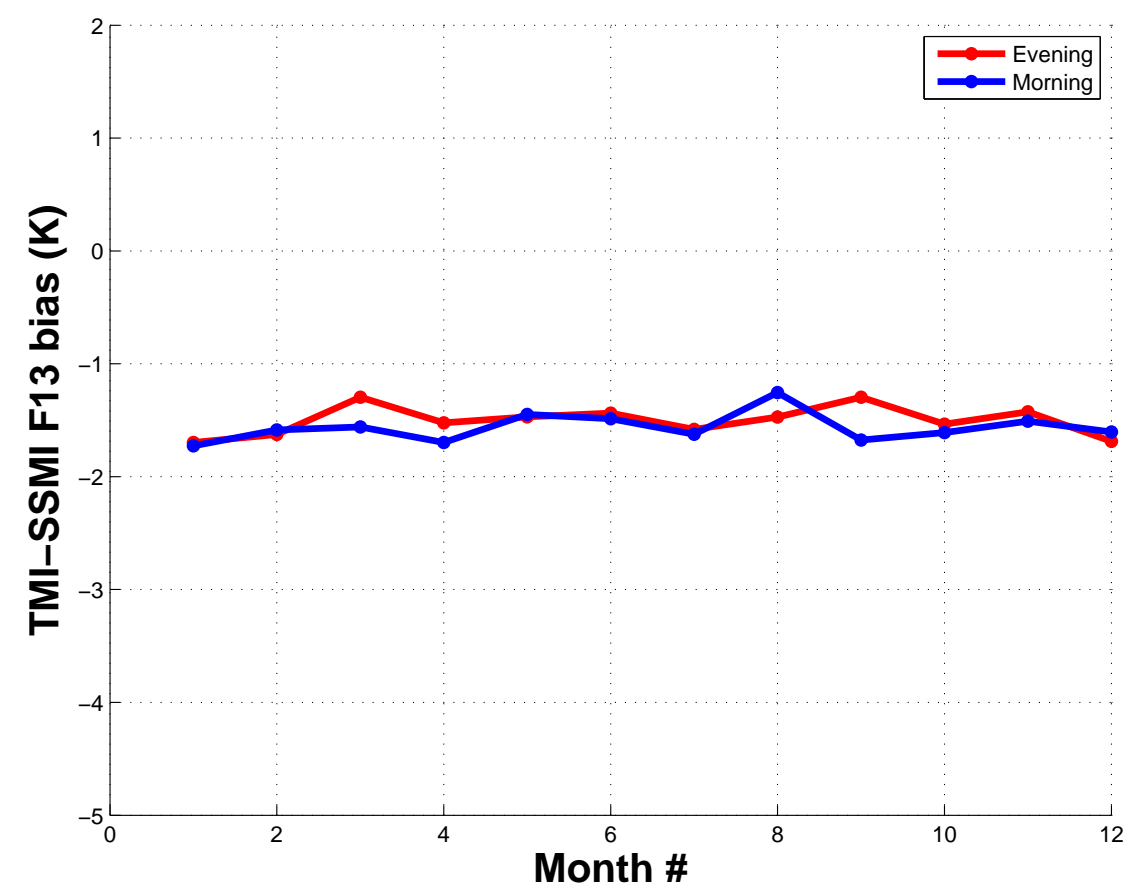

(a) $19 \mathrm{~V}$

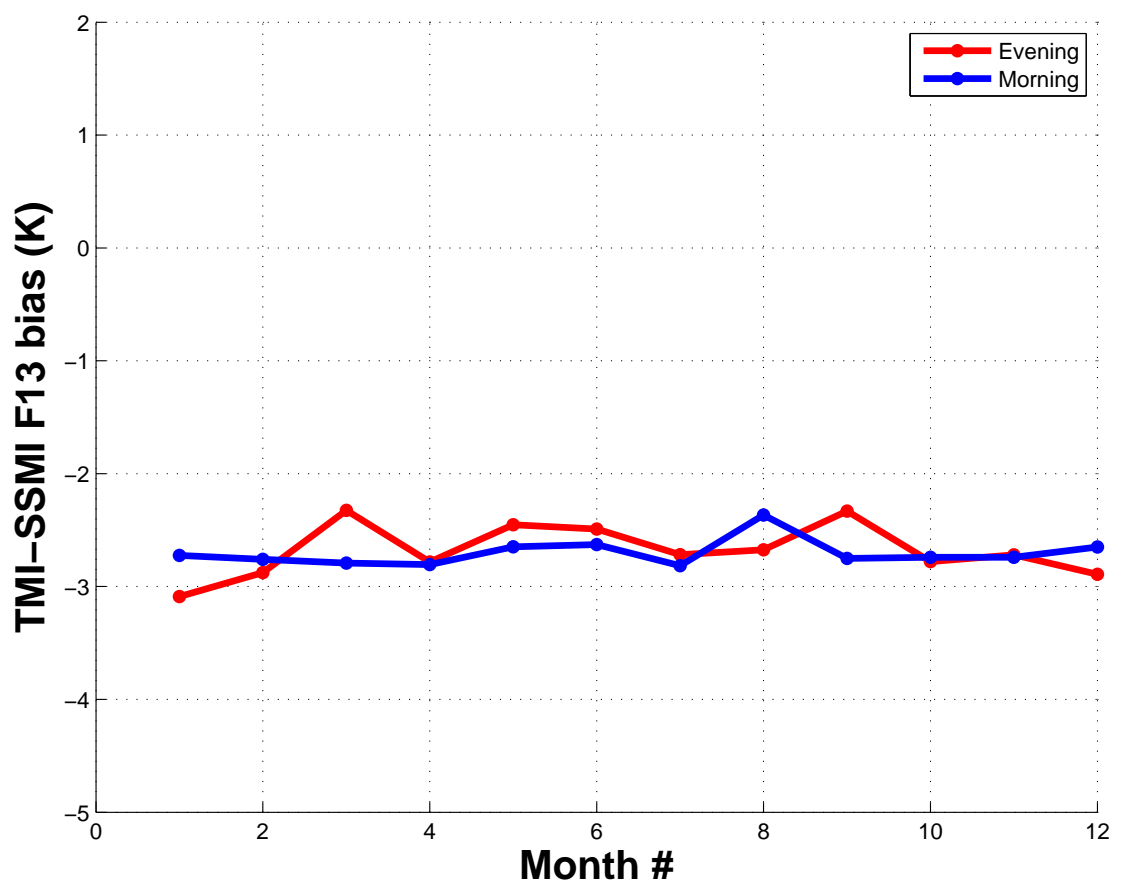

(b) $21 \mathrm{~V}$

Figure 3.21: Relative radiometric biases with SSMI F13 for corrected TMI Tb's. 




(c) $37 \mathrm{~V}$

Figure 3.21: Relative radiometric biases with SSMI F13 for corrected TMI Tb's. 


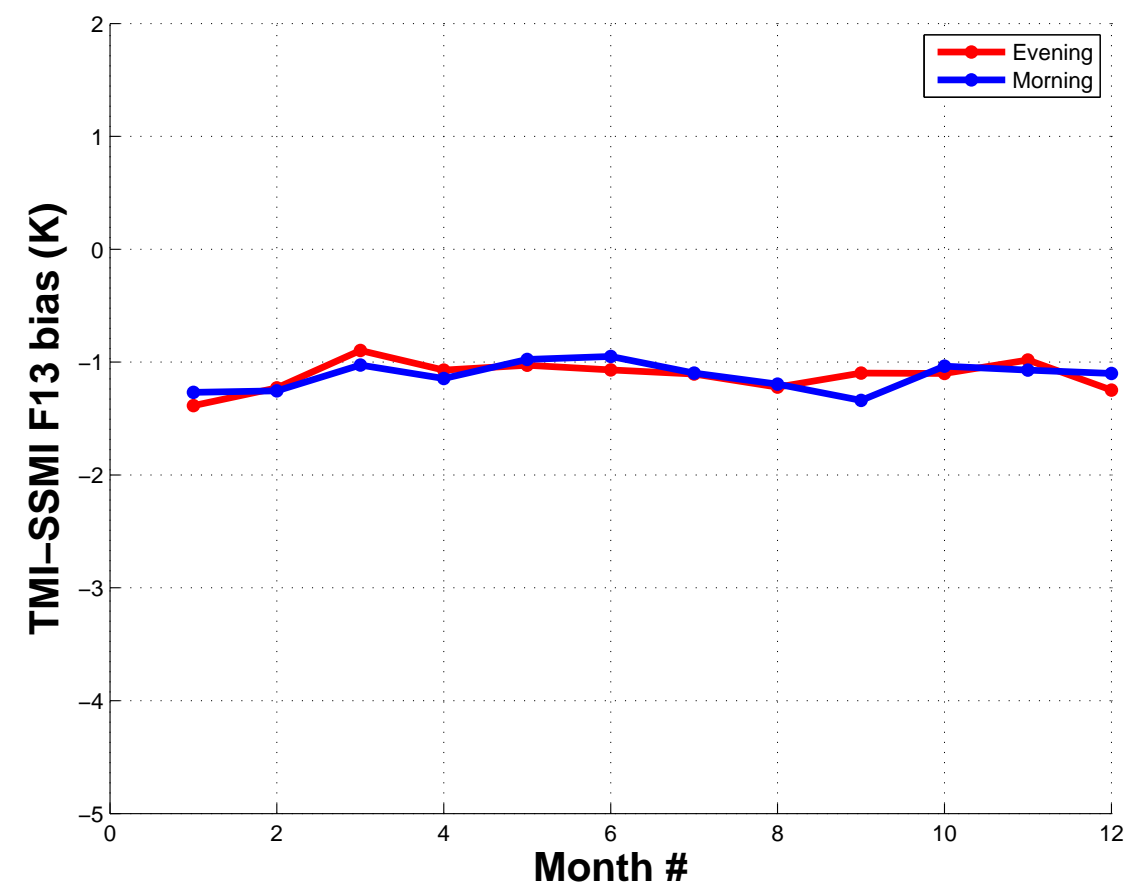

(a) $19 \mathrm{~V}$

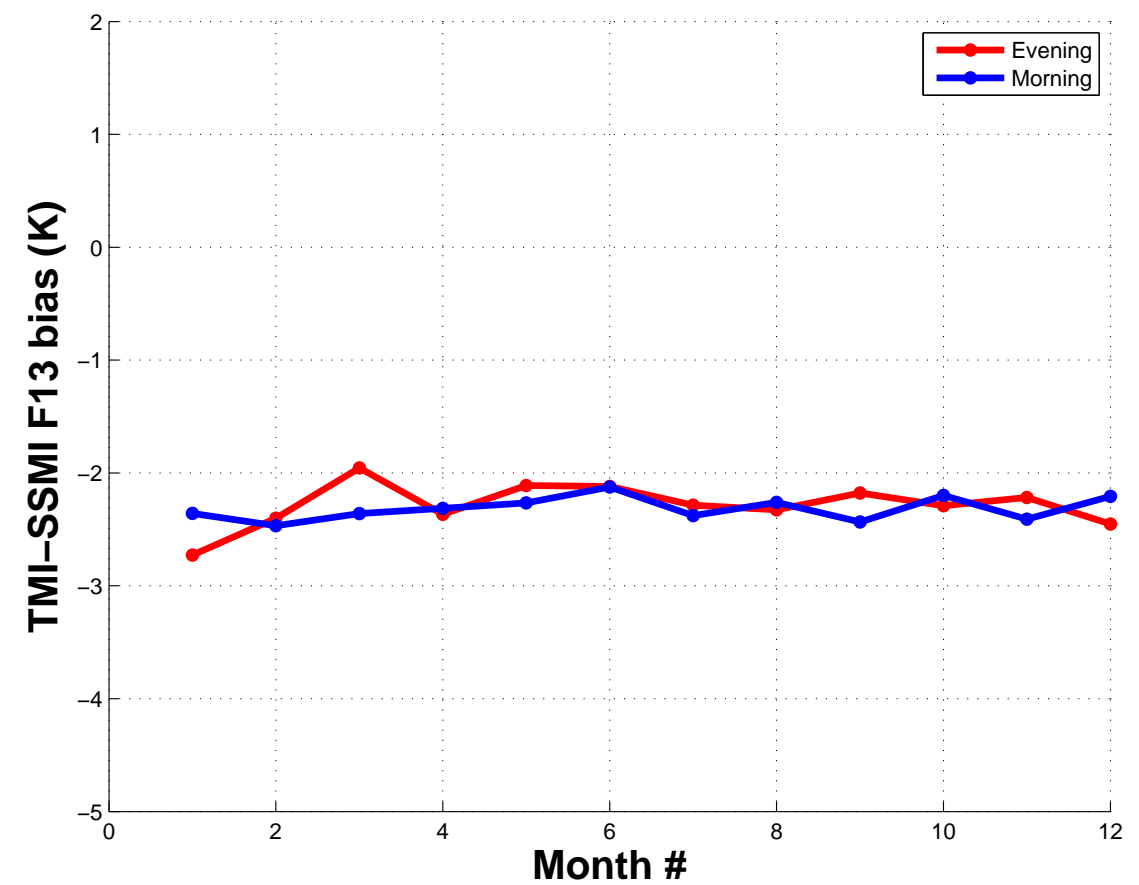

(b) $21 \mathrm{~V}$

Figure 3.22: Relative radiometric biases with SSMI F14 for corrected TMI Tb's. 


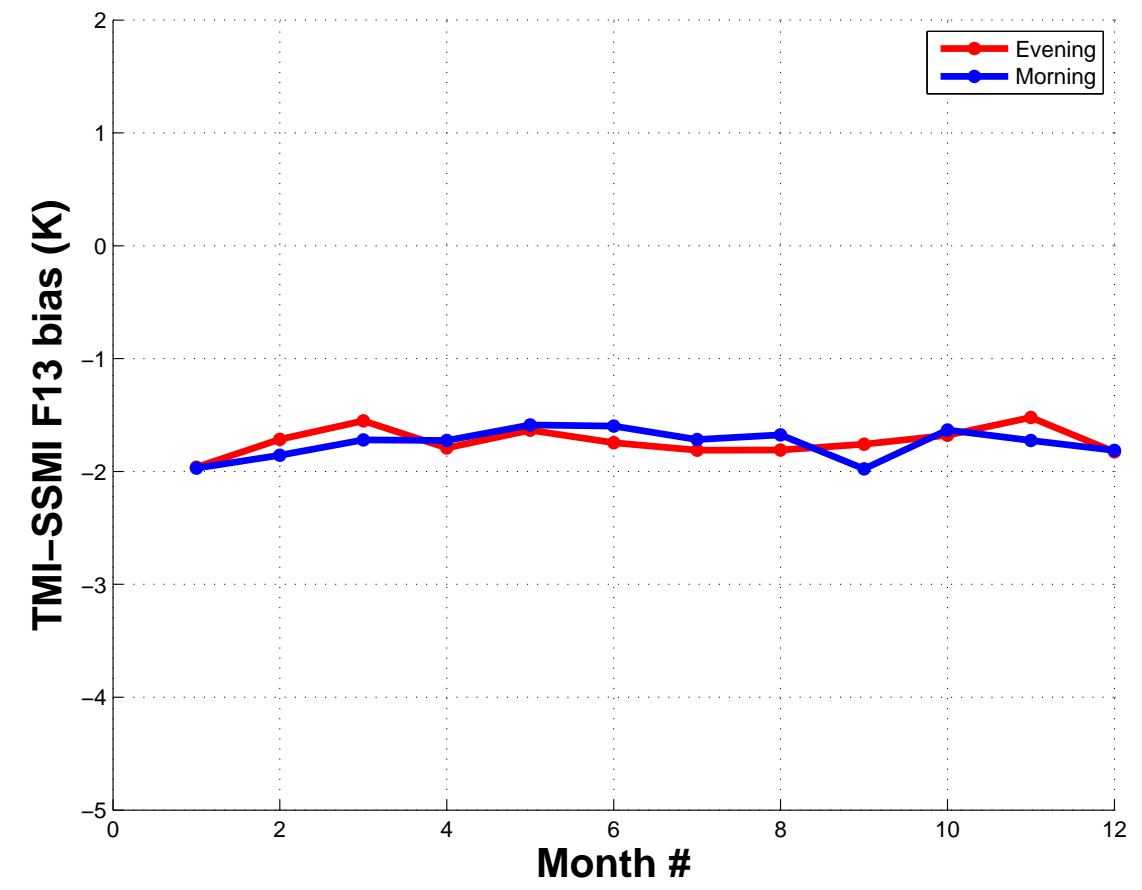

(c) $37 \mathrm{~V}$

Figure 3.22: Relative radiometric biases with SSMI F14 for corrected TMI Tb's. 


\section{SUMMARY AND CONCLUSIONS}

\subsection{TMI Tb Bias Errors}

Post-launch calibration of TMI by TRMM science team members revealed an apparent emissivity in the main reflector antenna. Since the TMI reflector physical temperature is not measured, a constant correction based on the estimated mean orbit physical temperature of the main reflector was applied to the 1B11 data products. During this dissertation research, inter-calibration of TMI with WindSat and SSMI revealed a time of day dependence in the radiometric calibration of TMI. Further investigation led to the discovery that the original TMI correction produced a time-variable Tb bias, which resulted in a systematic brightness temperature error with the period of one orbit. The cause of this error was the instantaneous variation of the on-orbit reflector physical temperature about the mean reflector temperature (assumed in the 1B11 correction).

Since the reflector bias is primarily caused by solar heating, the dynamic range of the bias also varies seasonally. Additionally, the heating and cooling of the reflector over an orbit follows a systematic pattern based on orbit time; therefore, the TMI brightness temperature bias is strongly correlated with latitude over short time periods ( $\sim 2-3$ days). Thus, the systematic nature of the TMI reflector bias has the potential of introducing diurnal, seasonal and regional biases in climatological studies performed using geophysical retrievals obtained from TMI observations. Further, since TMI observations are often included in data assimilation systems for numerical weather models; therefore, these time-variable radiometric errors also have the potential of introducing systematic errors in global weather predictions. In 
fact, Geer [34] states that TMI observations have currently been omitted from the ECMWF 4-D-var assimilation system, due to its time-varying calibration.

This dissertation recommends an empirical correction using TMI measured and radiative transfer theory modeled brightness temperatures in $1^{\circ}$ latitude by longitude boxes for an entire year. The recommended $\mathrm{Tb}$ correction removes the time of day dependence of the relative radiometric calibration between TMI and both WindSat and SSMI for all channels. The physical basis for this time-variable radiometric calibration error is provided by demonstrating the strong correlation of the $\mathrm{Tb}$ bias orbital signature with the expected variation of the TMI reflector physical temperature. Over each orbit, the estimated Tb bias drops sharply after the TRMM spacecraft enters the earth's shadow and rises after it exits the earth's shadow and is exposed to sunlight. This is consistent with the expected pattern of the reflector temperature over an orbit.

The daily average $\mathrm{Tb}$ bias patterns are given in Fig. 4.1 for 16 of the 47 day orbit precession period for TRMM during July 1 - July 16, 2005 [36]. This figure, with sets of 4 consecutive days plotted together, illustrate the $\mathrm{Tb}$ bias oscillations on a daily basis, where the bold lines indicate the period when TRMM is in the earth's shadow. The shape of the $\mathrm{Tb}$ bias orbital patterns are generally consistent (cooling during eclipse and warming during sunlight), but the peak to peak magnitude of these oscillations varies significantly. Figure 4.1a shows that the Tb biases for July 1-4 have a dynamic range from approximately $-0.2 \mathrm{~K}$ to $1.5 \mathrm{~K}$. On the other hand, the biases from July 13-16 extend from approximately -1K to 1K, as shown in Fig. 4.1d. Also, after July 8th, there are indications that the reflector temperature has reached an equilibrium temperature during the last 20 mins of the orbit. 
During the next 16 days, the daily average Tb bias orbital patterns for July 17 August 1, 2005 show a significant shape change as illustrated in Fig. 4.2. For example, the orbital biases for July 17 - July 20 remain essentially constant for a period of approximately 20 minutes after TRMM exits the earth's shadow. Gopalan et al. $[37\rfloor$ provides evidence that this behavior of the $\mathrm{Tb}$ bias is consistent with the expected reflector physical temperature, which remains cold during this post-eclipse period due to shadowing of the TMI by the TRMM spacecraft structure. Additionally, the bias pattern for August 1, the day that the TRMM $180^{\circ}$ yaw maneuver was performed (direction of flight reversed), is significantly different from the bias patterns of the previous days. On this day, the Tb bias rises immediately after TRMM exits the earth's shadow, as the TMI reflector is no longer shadowed by the spacecraft (see Fig. 3.17). Finally, the remainder of the 47 day cycle is given in Fig. 4.3. which shows the daily orbital patterns for August 2 - August 17, 2005. Here the Tb biases patterns, from August 2 - August 5, have a sharp decrease $\sim 15$ minutes before TRMM goes into eclipse, which is an effect of shadowing of TMI by the spacecraft and which leads to a cooling of the reflector physical temperature. After this period, there is another dramatic change in the $\mathrm{Tb}$ bias pattern, and for the remaining days the $\mathrm{Tb}$ bias patterns have similar shapes to those in Fig. 4.1. d. 




(a) July 1, 2005 - July 4, 2005

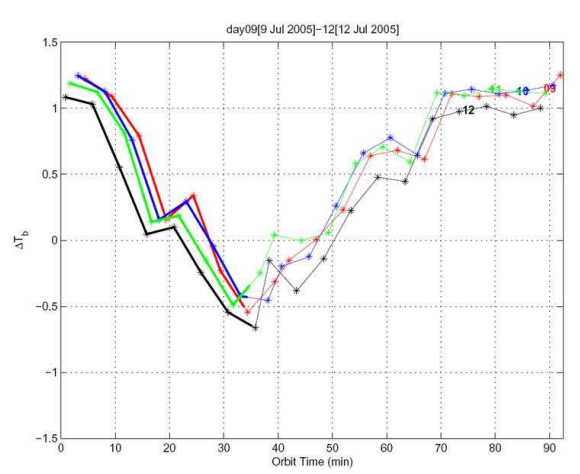

(c) July 9, 2005 - July 12, 2005

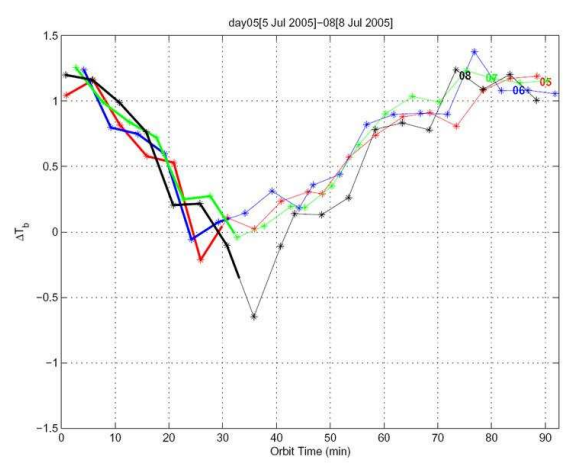

(b) July 5, 2005 - July 8, 2005

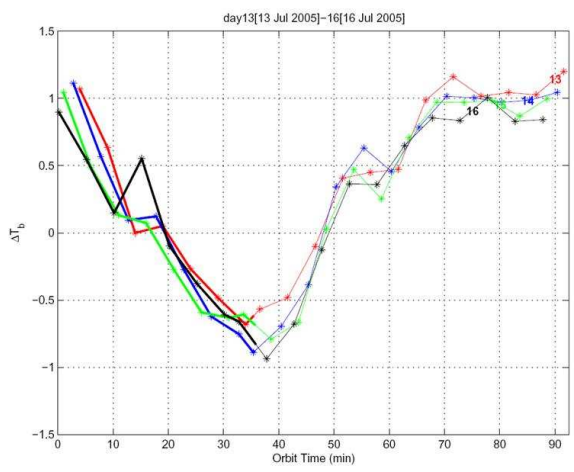

(d) July 13, 2005 - July 16, 2005

Figure 4.1: Daily estimated TMI Tb bias patterns. 


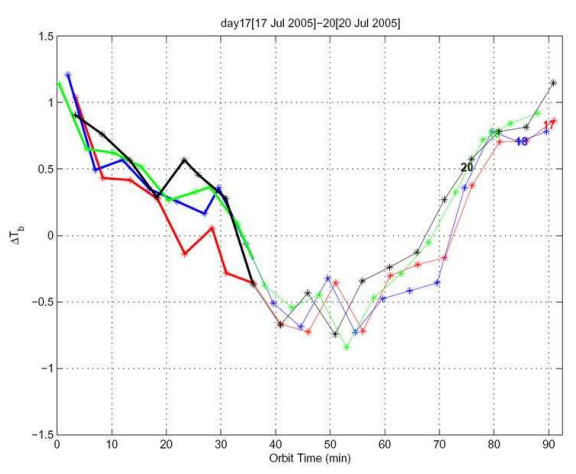

(a) July 17, 2005 - July 20, 2005



(c) July 25, 2005 - July 28, 2005



(b) July 21, 2005 - July 24, 2005



(d) July 29, 2005 - August 1, 2005

Figure 4.2: Daily estimated TMI Tb bias patterns. 




(a) August 2, 2005 - August 5, 2005

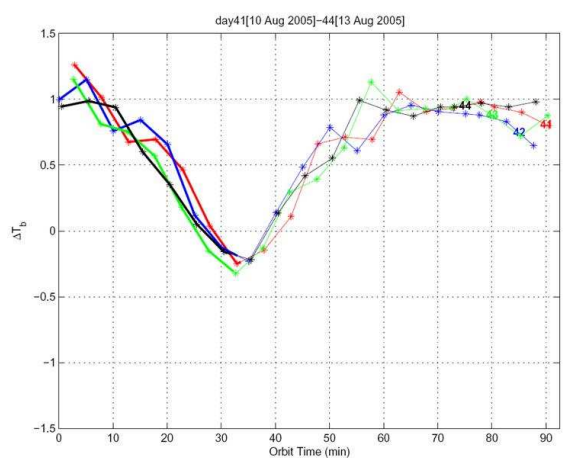

(c) August 10, 2005 - August 13, 2005

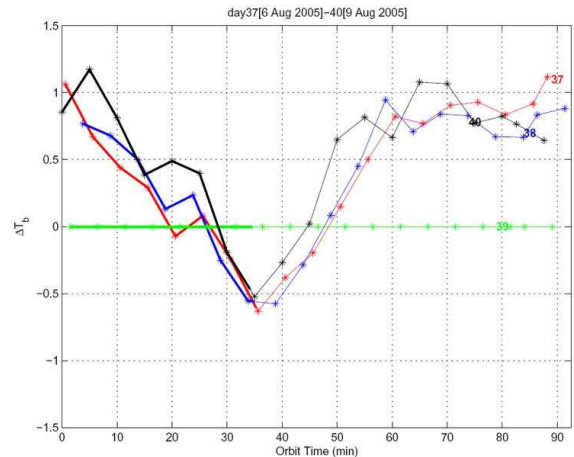

(b) August 6, 2005 - August 9, 2005

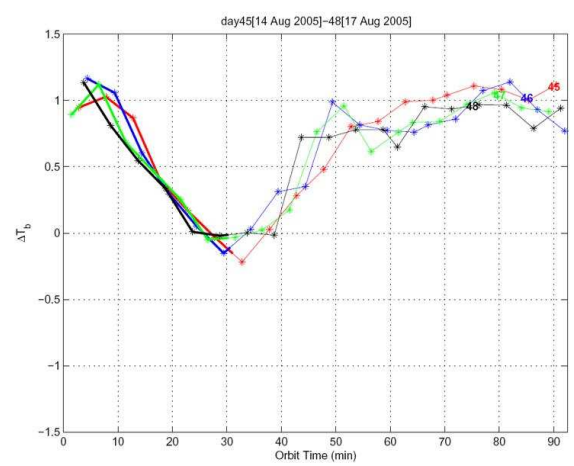

(d) August 14, 2005 - August 17, 2005

Figure 4.3: Daily estimated TMI Tb bias patterns. 
Because of these significant differences in the peak to peak variation and shape of the daily orbital bias patterns, the TMI Tb bias cannot be accurately modeled solely as a function of orbital time. Moreover, the daily orbital patterns do not repeat consistently over the TRMM precession period of 47 days, and they do not have a repeatable annual cycle. While daily bias estimates can be generated for a limited time period, this method is not feasible for operationally removing the time-varying TMI bias in near-real time during the generation of the 1B11 Tb data product. Further, the GDAS environmental parameters, which are necessary inputs to the RTM, are not available before 2000. Changing from GDAS to an earlier numerical weather analysis could introduce unknown offsets in the $\mathrm{Tb}$ bias patterns, which could be very undesirable for the long-term climate record. Thus, the time-varying bias estimation cannot be applied in the current form to future TMI data, as the bias values as a function of orbit time are not predictable on a daily basis.

\subsection{TMI Radiometric Correction}

Future ICWG activity will be to "stabilize" the TMI time-variable radiometric calibration, using techniques developed under this dissertation. Fortunately, the limitation in being able to predict the $\mathrm{Tb}$ bias orbital patterns is overcome by modeling the biases as a function of solar beta angle and orbit time after eclipse, as described in Gopalan et al. [37]. This provides a robust correction technique that can eliminate the TMI time-varying reflector bias for the entire lifetime of the TRMM mission, and the recommended correction will be applied to version 7 of the TRMM 1B11 data product that is scheduled to be released in 2010 . 
The correction to the 1B11 data product will be applied as a single additive Tb bias $(\Delta T b)$ to each brightness temperature measurement (pixel) in a single scan (independent of the channel frequency) [38]; however, different values are provided for the vertical and horizontal polarizations. The bias is modeled as a function of solar beta angle $(\beta)$ and orbit time since start of eclipse $\left(t_{e}\right)$. Thus for each scan,

$$
\begin{aligned}
\Delta T b & =f\left(\beta, t_{e}, \text { polarization }\right) \\
T b_{\text {corr }} & =T b_{1 B 11}+\Delta T b
\end{aligned}
$$

Where

$$
\begin{aligned}
& T b_{c o r r}=\text { Corrected brightness temperature } \\
& T b_{1 B 11}=\text { Original TMI 1B11 brightness temperature } \\
& \Delta T b=\text { brightness temperature bias value }
\end{aligned}
$$

This function $f\left(\beta, t_{e}\right.$, polarization $)$ is implemented as a three dimensional look-up table, with the three dimensions being $\beta, t_{e}$ and polarization respectively. An extra dimension to this table will be added to account for the pre-boost and post-boost orbit altitude differences in TMI 1B11 brightness temperature biases. The Tb bias look-up table will be generated from a four-year database of radiometric biases produced in $1^{\circ}$ boxes grouped along with their corresponding solar beta angle and orbit time since eclipse start. This database is quantized with respect to both solar beta angle and orbit time since eclipse start; each 
of the $\left(\beta, t_{e}\right)$ bins is averaged to generate a raw look-up table. This raw look-up table is then filtered to remove outliers and over-sampled to produce a "smoothed" final table, with negligible quantization errors. The beta step will be $0.25^{\circ}$ and time step will be 1 minute.

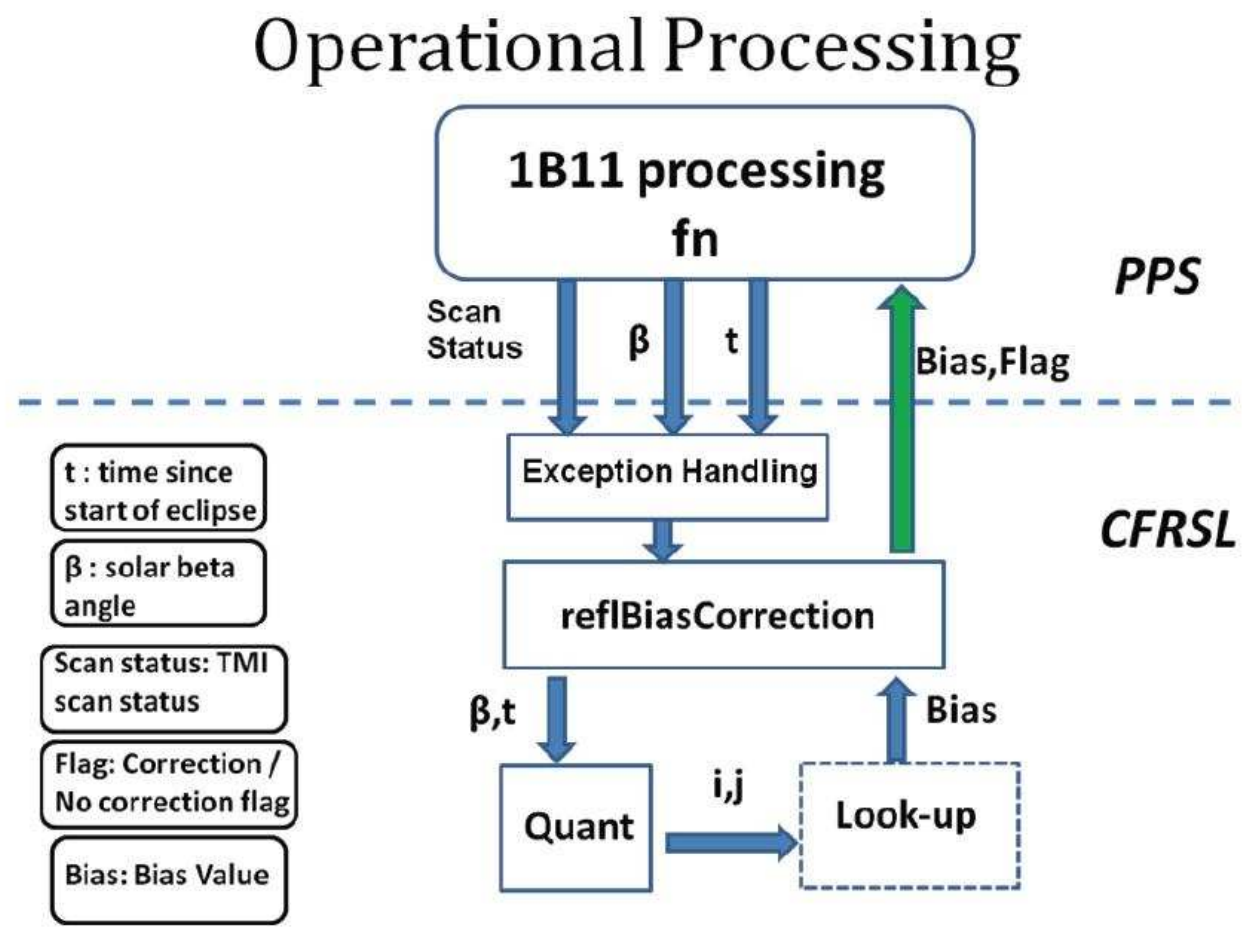

Figure 4.4: TRMM 1B11 version 7 bias correction. 
A simplified block-diagram illustrating the algorithm flow is shown in Fig. 4.4. The 1B11 processing software calls the CFRSL provided TMI radiometric correction function and passes the input parameters: scan status (quality flags), solar beta angle and orbit time since start of eclipse. First, the input data is validated based on scan status information and limits of $\beta$ and $t_{e}$. These validated input parameters are quantized to generate indices $(i, j)$ for the look-up table. The bias values (vertical and horizontal) corresponding to the equivalent indices are outputted from the look-up table, and a bias status quality flag is also generated along with the bias value. Only the brightness temperatures with valid scan status will generate a bias correction, and any out-of-limit values of $\beta$ and/or $t_{e}$ will result in no correction.

\subsection{Error Sources}

This section discusses the major error sources involved in the TMI Tb bias estimation. First, the TMI radiometer has brightness temperature measurement errors (NEDT) of about $\pm 0.5 \mathrm{~K}$ for the low frequency channels and up to $\pm 0.93 \mathrm{~K}$ for the $85 \mathrm{GHz}$ channels. When averaging the $25-100 \mathrm{~Tb}$ 's, which occur in a $1^{\circ}$ box, these values become negligibly small. On the other hand, the $\mathrm{Tb}$ observations in each 1 degree bin are not uniform because of spatial variability of the environmental scenes and can have standard deviations of up to $2 \mathrm{~K}$ for the vertical polarization and up to $3 \mathrm{~K}$ for horizontal polarization for rain-free ocean scenes. Fortunately, these errors are observed to be near-zero mean Gaussian random variables, which increase the uncertainty of individual $\mathrm{Tb}$ samples but does not affect the mean bias estimates. 
Considering the RTM calculation of TB, the ocean emissivity has a slight dependence on the wind direction relative to the observation azimuth, which has been ignored. Given that the ocean wind direction pdf is approximately uniform, the emissivity anisotropy results in a zero mean value when averaged over all directions. Also, the Tb measurements in the matchup dataset have a maximum temporal difference of 3 hour from the time of the corresponding GDAS environmental parameter estimate. It is probable that the environmental conditions at the ocean scene (primarily wind speed, water vapor and cloud liquid water) would change slightly in this time period, leading to an error in the relative bias estimate. Further, the GDAS atmospheric inputs to the RTM, such as the water vapor and cloud liquid water profiles, are likely to contain significant errors, which also lead to random errors in the relative bias estimates. The RTM modules used by the ICWG are also imperfect and are likely to introduce small errors in the bias estimates. Fortunately, the large number of $1^{\circ}$ boxes that are averaged to derive a $\mathrm{Tb}$ bias helps to minimize the effects of these error sources. Experience shows the combination of these error sources to be a zero mean gaussian error pdf about the "true" value. Moreover, these errors caused by the RTM modeling errors are uncorrelated with orbital time, which should tend to be reduced by averaging. Thus, while the recommended correction is capable of stabilizing the TMI time-variable radiometric calibration; however, it cannot completely eliminate the DC bias in the TMI calibration.

Further, the TMI time-varying bias has slight frequency dependence between radiometer channels (the estimated reflector emissivity varies from 0.0370 to 0.0377 in the low frequency channels [7]); and the correction recommended in this dissertation does not account for these small differences. The empirical correction described in this dissertation 
should be considered a "first-order" correction that eliminates gross time-varying calibration biases in TMI, but it may not improve the more subtle variations in the TMI calibration. Additional work is required to eliminate these subtle calibration biases.

\subsection{Future Work}

Future calibration efforts for TMI, and other similar radiometers, can improve relative bias estimates by reducing the errors in the RTM modeled values. Careful analysis of the differences between RTM predictions and observed Tb's for multiple radiometers in all environmental conditions is required. Such an analysis is likely to yield insights into the causes and the extent of RTM Tb errors. The empirical technique used to estimate the TMI time-varying bias can be repeated for WindSat and SSMI; and any similar time-varying radiometric calibration errors existing in WindSat and SSMI can be corrected using this procedure.

The inter-calibration analysis performed in this dissertation confirmed the presence of a time-varying calibration bias in TMI. Similar analyses should be performed during the post-launch calibration of other non-sun-synchronous satellite radiometers in the GPM era.

The future Indo-French satellite radiometer MADRAS, which will fly as part of the MeghaTropiques mission [39], is a good candidate for such inter-calibration analysis.

Finally, the GPM mission aims to attain a calibration accuracy of $0.1 \mathrm{~K}$ for the radiometers in its constellation. The future research activities suggested above, along with other research efforts, will be necessary to meet this challenging goal. 
APPENDICES 


\section{A SATELLITE RADIOMETERS IN THE ICWG INTER-CALIBRATION DATASET}

\section{A.1 Special Sensor Microwave Imager (SSMI)}

The first SSMI radiometer was launched in July 1987 as part of the Defense Meteorological Space Program (DMSP) [40]. There have been 6 SSMI radiometers launched on a series of polar orbiting DMSP satellites as shown in Table A.1 :

Table A.1: DMSP satellites with SSM/I radiometers

\begin{tabular}{|c|c|}
\hline F13 SSM/I & May 1995 to present \\
\hline F14 SSM/I & May 1997 to present \\
\hline F15 SSM/I & Dec 1999 to Aug 2006 \\
\hline
\end{tabular}

SSMI follows a near circular, sun synchronous, polar orbit with a conical scan at an incidence angle of 53.4 degrees and makes vertical and horizontal polarization measurements at $19.35,37.0$, and $85.5 \mathrm{GHz}$, and a single vertical polarization measurement at $22.235 \mathrm{GHz}$. Figure A.1 depicts the scan geometry of the SSMI radiometer ${ }^{1}$. The high incidence angle allows SSMI to view a much larger coverage area per scan compared to low incidence angle or zenith viewing instruments. SSMI operates in two scan modes; the $19 \mathrm{GHz}-37 \mathrm{GHz}$ channel measurements, which have relatively large footprint sizes, are made for every alternate pixel of every alternate scan while the $85 \mathrm{GHz}$ channel measurements are made for every pixel in every scan. Each SSMI scan takes 1.9 seconds, and measurements are taken over a scan angle of 102 degrees.

Table A.2 gives additional information about the footprint sizes and integration times of each channel.

\footnotetext{
${ }^{1}$ Figures A.1 and A.2 have been reproduced from Cavalieri $\lfloor 41$.
} 


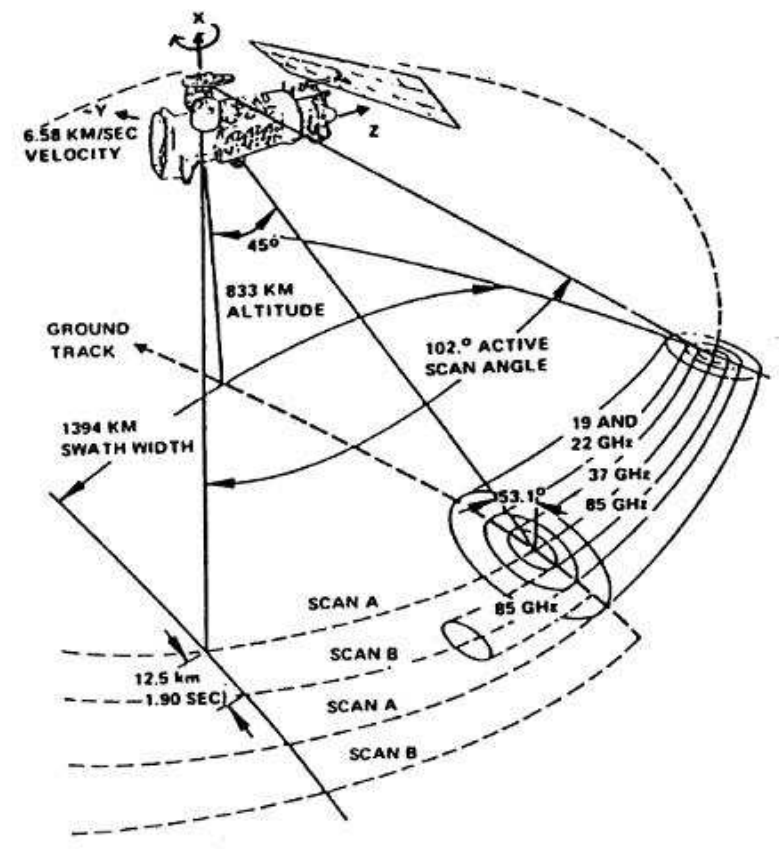

Figure A.1: SSMI scan geometry

Table A.2: SSMI Characteristics

\begin{tabular}{|c|c|c|c|}
\hline Center Freq.(GHz) & Polarizations & Integration period & EFOV $(\mathrm{km} \mathrm{x} \mathrm{km)}$ \\
\hline 19.35 & $\mathrm{~V}, \mathrm{H}$ & $7.95 \mathrm{~ms}$ & $69 \times 43$ \\
\hline 22.235 & $\mathrm{~V}$ & $7.95 \mathrm{~ms}$ & $60 \times 40$ \\
\hline 37 & $\mathrm{~V}, \mathrm{H}$ & $7.95 \mathrm{~ms}$ & $37 \times 29$ \\
\hline 85.5 & $\mathrm{~V}, \mathrm{H}$ & $3.89 \mathrm{~ms}$ & $15 \times 13$ \\
\hline
\end{tabular}

During the 258 degree part of each scan when the SSMI is not making active measurements, it views a calibration hot load and a cold load as shown in Fig. A.2. The hot load is a warm radiometric source with known physical temperature and an emissivity of almost unity. Calibration at the cold end is performed by viewing outer space, which has a stable radiometric emission of $2.7 \mathrm{~K}$.

SSMI is a total power radiometer, which means that the voltage output produced at its detector is directly proportional to the antenna temperature. Thus, the hot and cold 


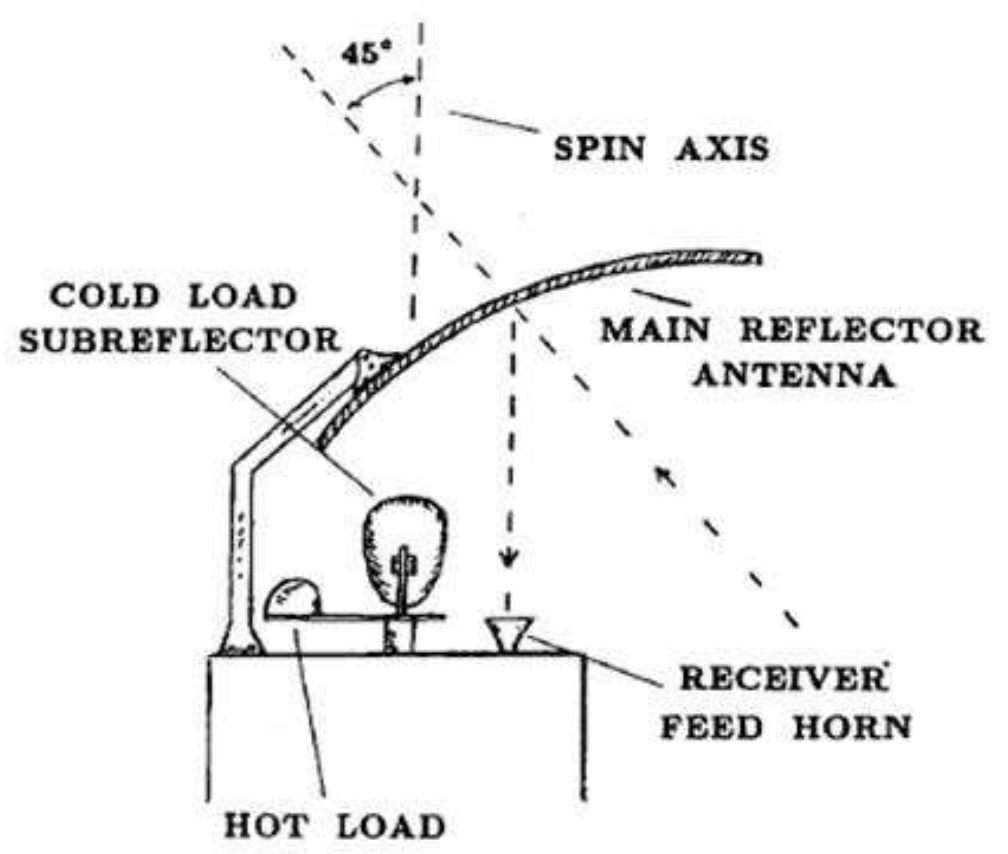

Figure A.2: SSMI reflector positioning

load measurements are used to set up a linear calibration fit to estimate the brightness temperature as follows $\lfloor 42\rfloor$ :

$$
T_{A}=\left(\frac{\bar{T}_{w}-T_{c}}{\bar{V}_{w}-\bar{V}_{c}}\right)\left(V_{A}-\bar{V}_{c}\right)+T_{c}
$$

where $T_{A}$ is the antenna brightness temperature; $\bar{T}_{w}$ is the mean warm load temperature; $T_{c}$ is the cold load temperature; $\bar{V}_{w}$ is the mean radiometric count (the integrated and digitized value of the radiance observed at the reflector antenna) for the warm load; $\bar{V}_{c}$ is the mean radiometric count for the cold load and $V_{A}$ is the scene radiometric count.

TMI and WindSat, which are described next, are also total power radiometers and have a similar on-board calibration equation. 


\section{A.2 TRMM Microwave Imager (TMI)}

TRMM is NASA's first mission dedicated to observing and understanding the tropical rainfall and how this affects the global climate. TMI is capable of quantifying the water vapor, the cloud water, and the rainfall intensity in the atmosphere by measuring the brightness temperatures of microwave emissions from the Earth. Another important feature of the TMI is that Sea Surface Temperatures can be estimated from its measurements, even through clouds, which are nearly transparent at $10.7 \mathrm{GHz}$. When it was launched in November 1997, the TRMM satellite followed a near circular, non-sun-synchronous orbit of approximately $350 \mathrm{~km}$ altitude with an inclination of 35 degrees. In August 2001 the satellite was boosted to an altitude of $402 \mathrm{~km}$ in an effort to increase its mission life, as the spacecraft required much more fuel to make orbit adjustments to counter effects of friction at the $350 \mathrm{~km}$ altitude. The TRMM orbit provides extensive coverage in the tropics and allows each location to be covered at a different local time each day over a period of approximately one month. The TMI is constructed similarly to the SSMI, with the addition of a $10.65 \mathrm{GHz}$ channel. The water vapor channel center frequency was also changed to $21.3 \mathrm{GHz}$ from $22.235 \mathrm{GHz}$ in SSMI. The TMI sensor package consists of two feedhorns, one containing the $10.7 \mathrm{GHz}$ $\mathrm{V}$ and $\mathrm{H}$ pol. channels and the other feedhorn containing the other 7 channels, as shown in Fig. A.3.

The instantaneous field of view (IFOV) of each channel is the footprint of the antenna half-power beamwidth on the earth's surface at a given instant. Table A.3 shows more information about TMI footprint sizes. 


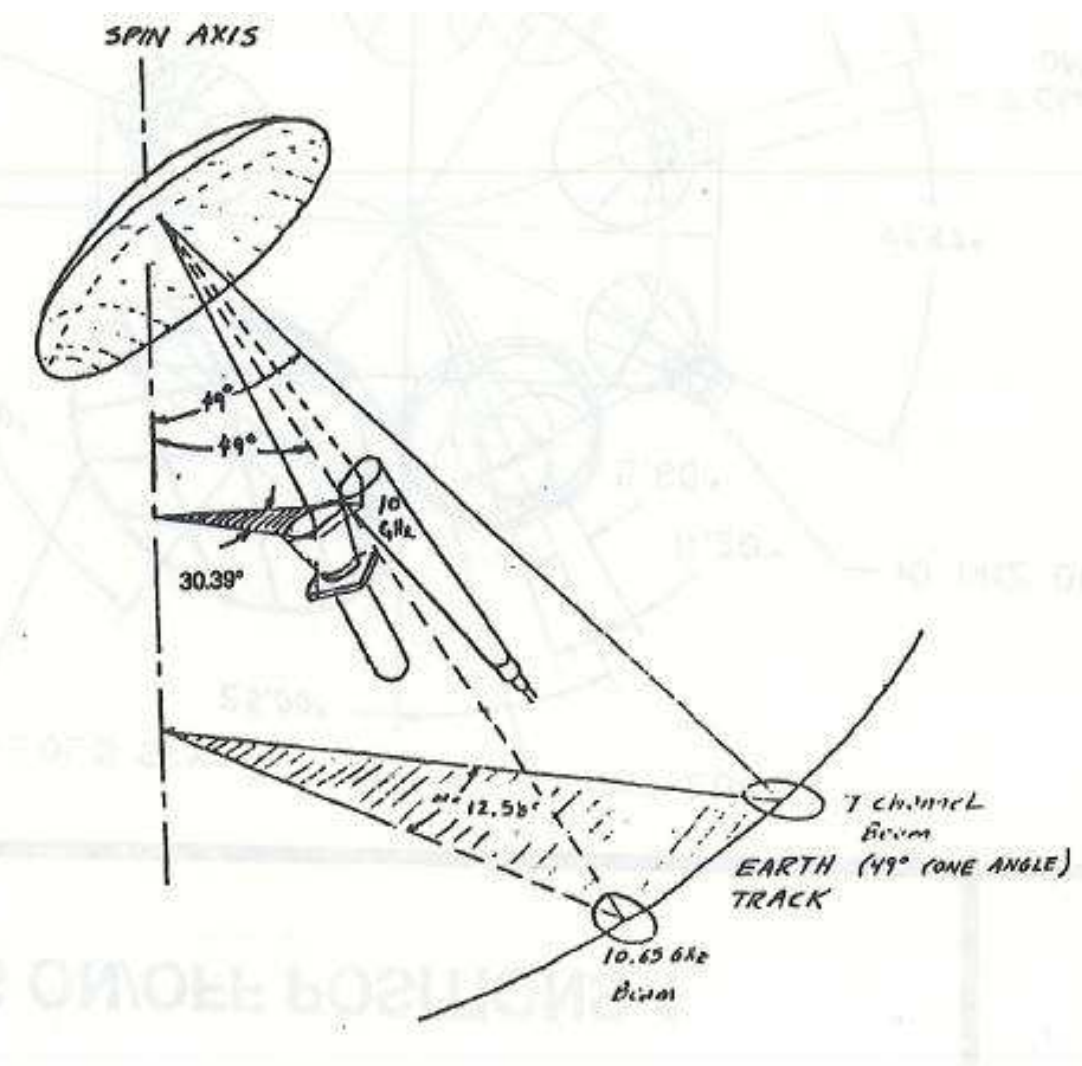

Figure A.3: TMI scan geometry

Table A.3: TMI Characteristics

\begin{tabular}{|l|c|c|l|l|}
\hline $\begin{array}{l}\text { Center } \\
\text { Freq.(GHz) }\end{array}$ & Polarizations & Samples per scan & $\begin{array}{l}\text { Integration time } \\
\text { per sample }(\mathrm{ms})\end{array}$ & $\begin{array}{l}\mathrm{EFOV}(\mathrm{km} \\
\mathrm{x} \mathrm{km})\end{array}$ \\
\hline 10.65 & $\mathrm{~V}, \mathrm{H}$ & 104 & 6.6 & $63 \times 37$ \\
\hline 19.35 & $\mathrm{~V}, \mathrm{H}$ & 104 & 6.6 & $30 \times 18$ \\
\hline 21.3 & $\mathrm{~V}$ & 104 & 6.6 & $23 \times 18$ \\
\hline 37 & $\mathrm{~V}, \mathrm{H}$ & 104 & 6.6 & $16 \times 9$ \\
\hline 85.5 & $\mathrm{~V}, \mathrm{H}$ & 208 & 3.3 & $7 \times 5$ \\
\hline
\end{tabular}

Since the TMI antenna rotates significantly over the integration time period, the Effective Field of View (EFOV) is defined as the ellipse at the center of the area covered during the sample integration time, as shown in Fig. A.4 ${ }^{2}$. The location of the center of the EFOV is provided in standard TMI products.

\footnotetext{
${ }^{2}$ Figure A.4 has been reproduced from Kummerow et. al. 43].
} 




Figure A.4: TMI footprint characteristics

The TMI feedhorns make radiometric measurements over a 130 degree swath in each scan, while also viewing the warm and cold calibration loads for $\sim 10$ degrees during each 
scan, as shown in Fig. A.5. The linear calibration function is calculated for each scan, using Equation A.1.

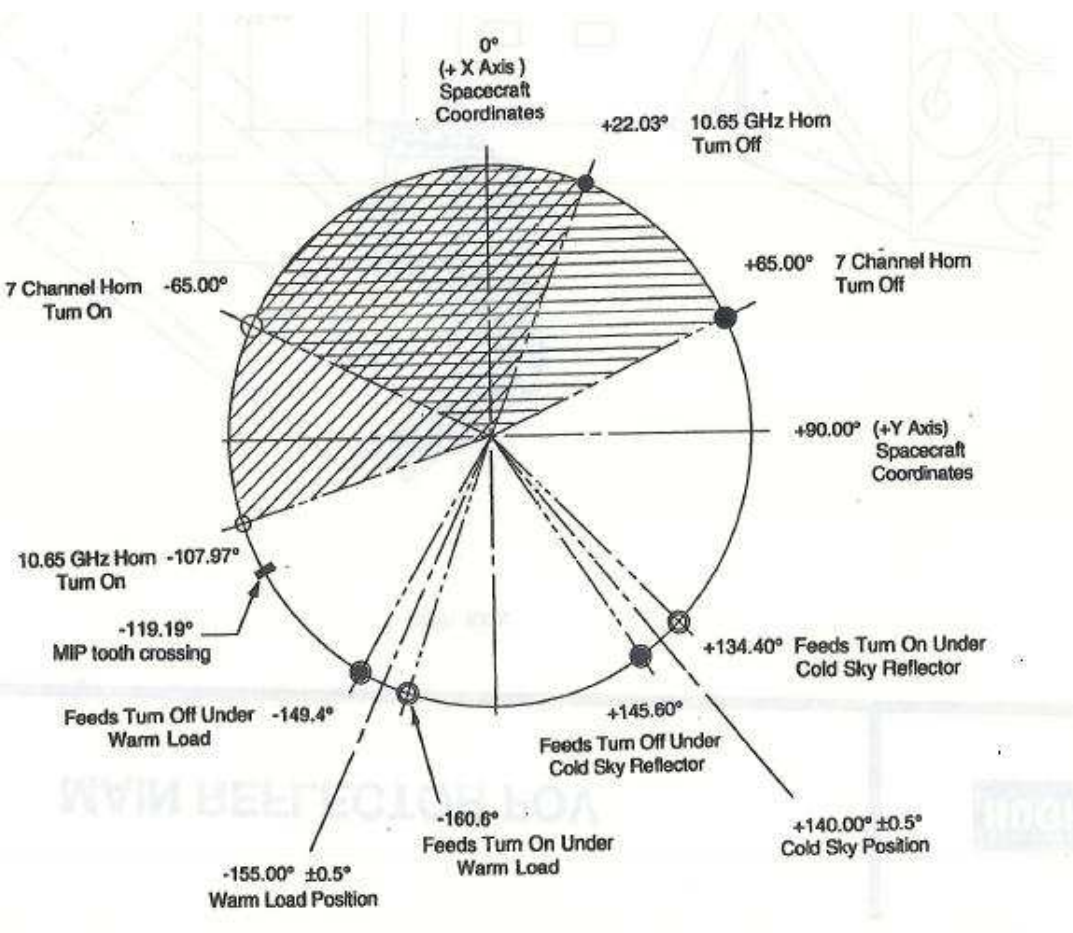

Figure A.5: Azimuth distribution of TMI scan sectors

\section{A.3 WindSat}

WindSat was primarily designed to measure the ocean surface wind vector from space.

It also measures sea surface temperature, total precipitable water, integrated cloud liquid water, and rain rate over the ocean. In addition to the environmental parameters mentioned above, WindSat data products also include Sea Ice retrievals and Snow and Soil Moisture retrievals [44].

The WindSat satellite has an inclination angle of 98.7 degrees and revolves around the earth at an $830-\mathrm{km}$ orbit. The antenna beams view the Earth at incidence angles ranging 
from 50 to 55 degrees. The WindSat radiometer operates at 6.8, 10.7, 18.7, 23.8, and 37 GHz. The 10.7, 18.7, and $37.0 \mathrm{GHz}$ channels measure all six principal polarizations, while the $6.8 \mathrm{GHz}$ channel and the $23.8 \mathrm{GHz}$ channel measure only the vertical and horizontal polarizations. Fig. A.6 shows the construction of the Windsat sensor assembly ${ }^{3}$. All the WindSat channels have a separate feedhorn for each pair of polarizations $(\mathrm{V} / \mathrm{H}, \mathrm{L} / \mathrm{R}$ circular and $+/-45)$; the WindSat feed horn array therefore contains 11 feedhorns. The WindSat channels have different incidence angles, as shown in Table. A.4, since the feedhorns for each channel are separated in the along-track direction.

Table A.4: WindSat Characteristics

\begin{tabular}{|c|c|c|l|}
\hline Center Freq.(GHz) & Polarizations & Incidence angle(deg) & $\begin{array}{l}\text { Horizontal reso- } \\
\text { lution }(\mathrm{km} \times \mathrm{km})\end{array}$ \\
\hline 6.8 & $\mathrm{~V}, \mathrm{H}$ & 53.5 & $40 \times 60$ \\
\hline 10.7 & $\mathrm{~V}, \mathrm{H}, \pm 45, \mathrm{~L}, \mathrm{R}$ & 49.9 & $25 \times 38$ \\
\hline 18.7 & $\mathrm{~V}, \mathrm{H}, \pm 45, \mathrm{~L}, \mathrm{R}$ & 55.3 & $16 \times 27$ \\
\hline 23.8 & $\mathrm{~V}, \mathrm{H}$ & 53.0 & $12 \times 20$ \\
\hline 37 & $\mathrm{~V}, \mathrm{H}, \pm 45, \mathrm{~L}, \mathrm{R}$ & 53.0 & $8 \times 13$ \\
\hline
\end{tabular}

\footnotetext{
${ }^{3}$ Figure A.6 has been reproduced from Gaiser et. al. 45.
} 


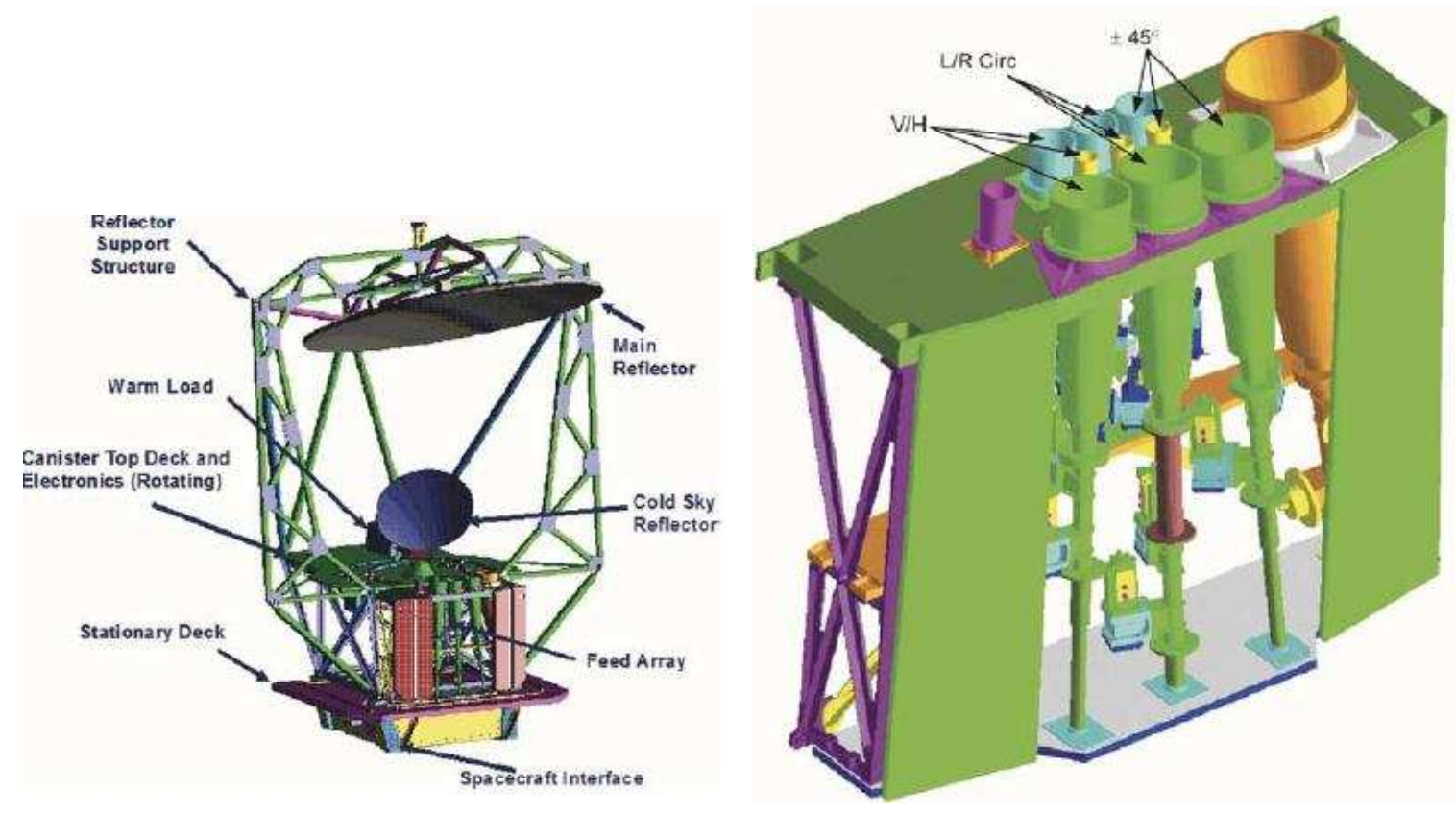

Figure A.6: WindSat sensor assembly 


\section{B NORMALIZATION OF WINDSAT TB'S TO TMI FREQUENCY AND INCIDENCE ANGLES}

The Tb normalization between TMI and WindSat for all the TMI channels is illustrated graphically in the following figures. Panel-(a) in each figure is a scatter diagram of binned averages of "raw" WindSat 10V and collocated TMI measurements for the corresponding channel; and the dashed line depicts the $45 \mathrm{deg}$ line where TMI and WindSat measurements are equal. The predicted $\mathrm{Tb}$ difference due to the frequency and incidence angle differences between the two channels is shown in panel-(b). The results of normalizing the WindSat Tb according to Eqn. (2.4) are presented in panel-(c) in each figure. 


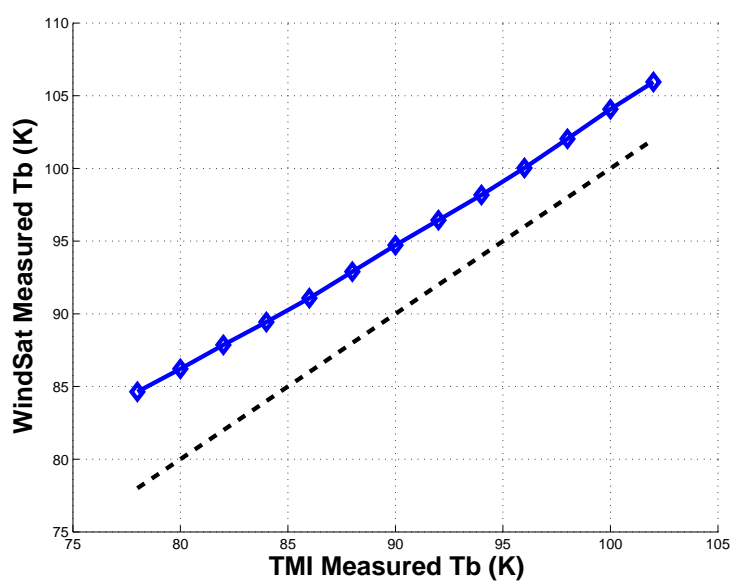

(a) Raw Tb observations.

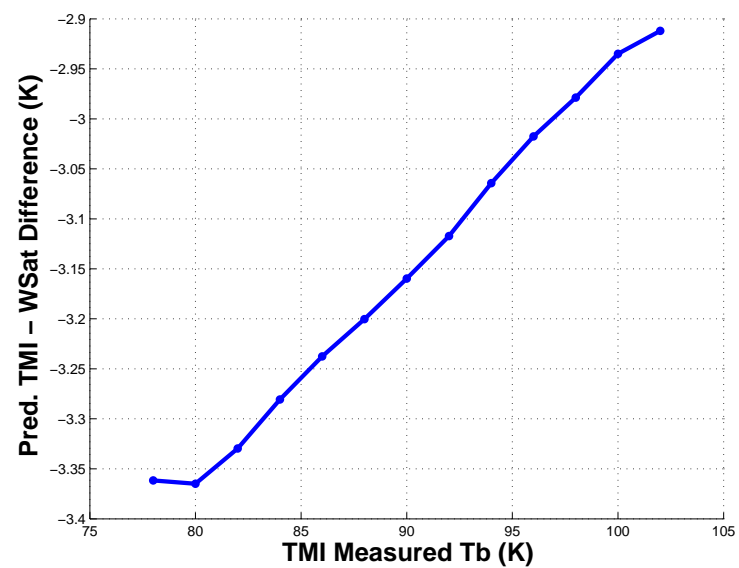

(b) Expected Tb difference predicted by RTM.

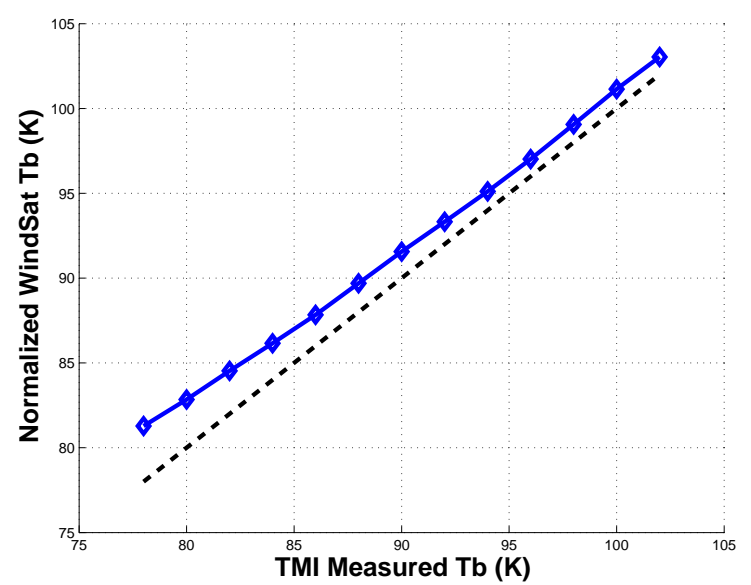

(c) Normalized WindSat and TMI Tb comparisons.

Figure B.1: Normalized WindSat 10H Tb observations to corresponding TMI channel frequency and incidence angle. 


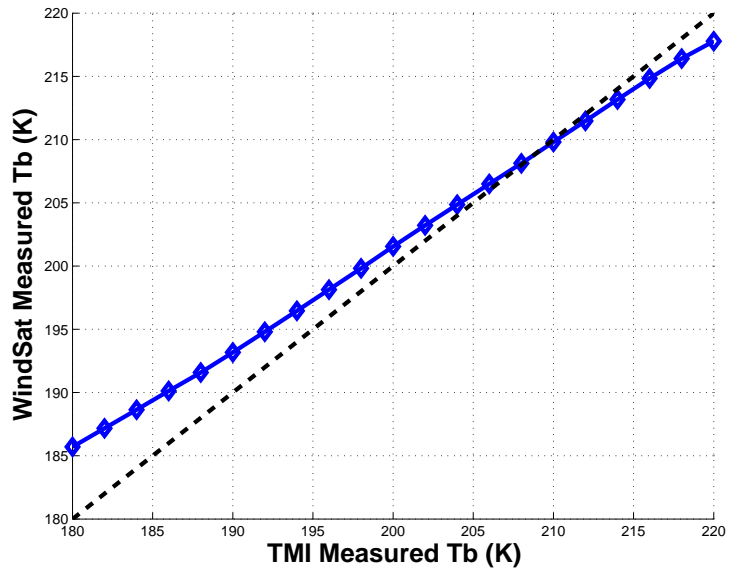

(a) Raw Tb observations.

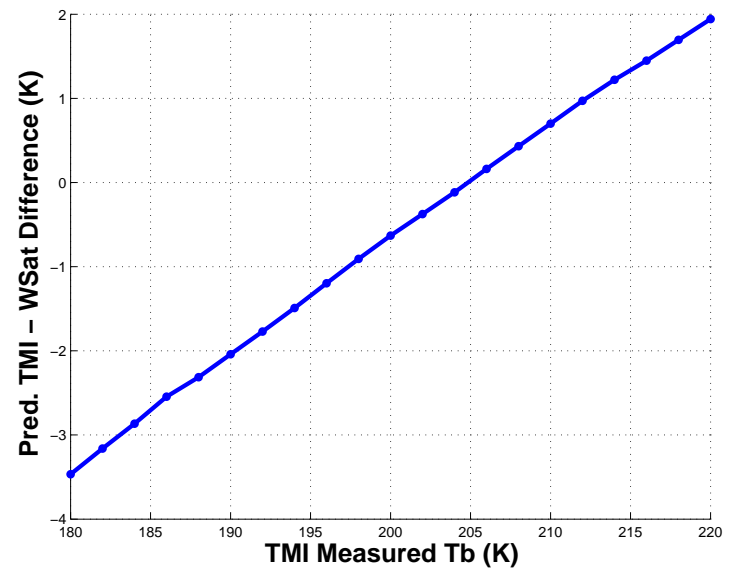

(b) Expected Tb difference predicted by RTM.

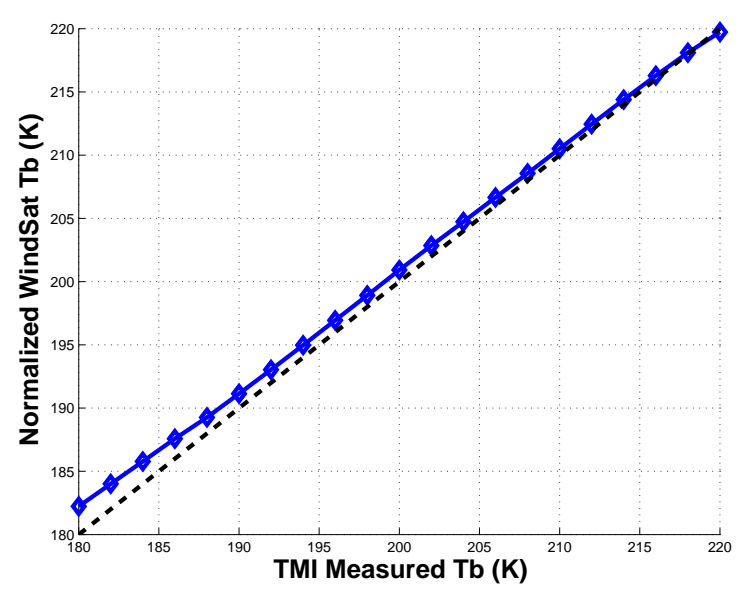

(c) Normalized WindSat and TMI Tb comparisons.

Figure B.2: Normalized WindSat 18V Tb observations to corresponding TMI channel frequency and incidence angle. 


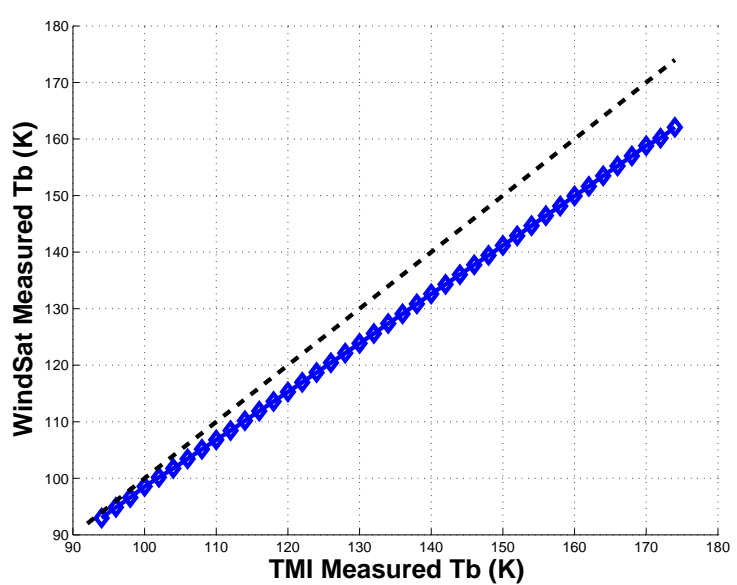

(a) Raw Tb observations.

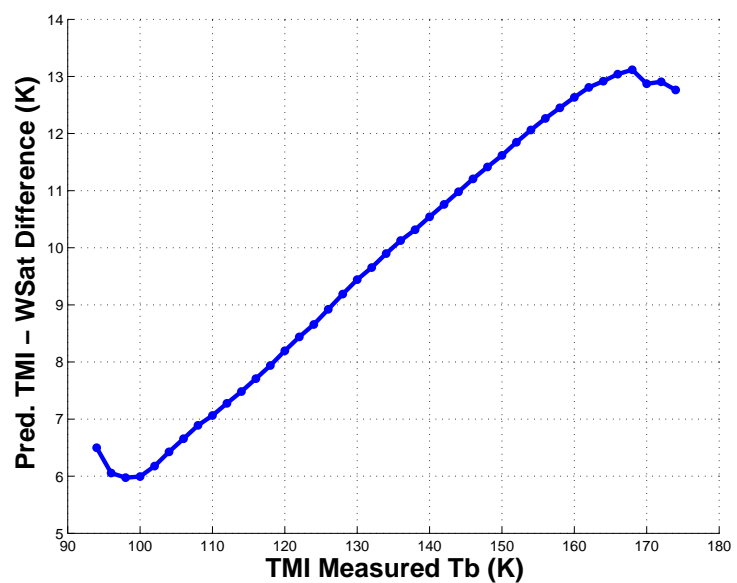

(b) Expected $\mathrm{Tb}$ difference predicted by RTM.

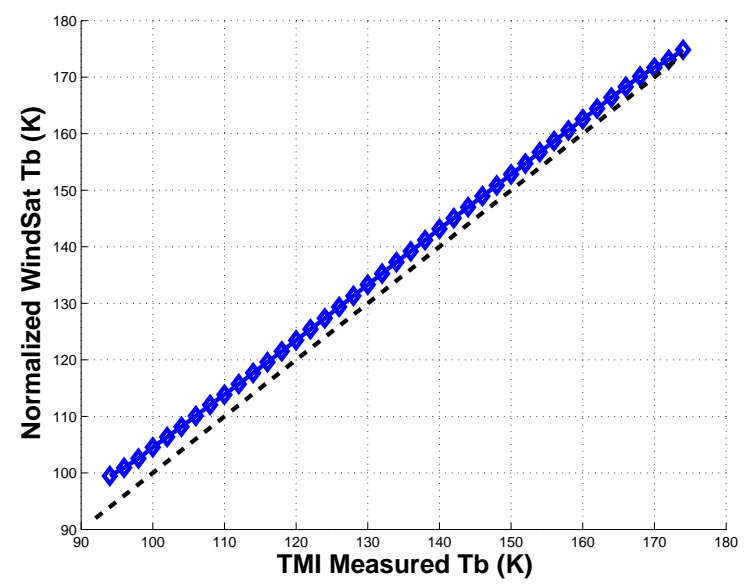

(c) Normalized WindSat and TMI Tb comparisons.

Figure B.3: Normalized WindSat 19H Tb observations to corresponding TMI channel frequency and incidence angle. 


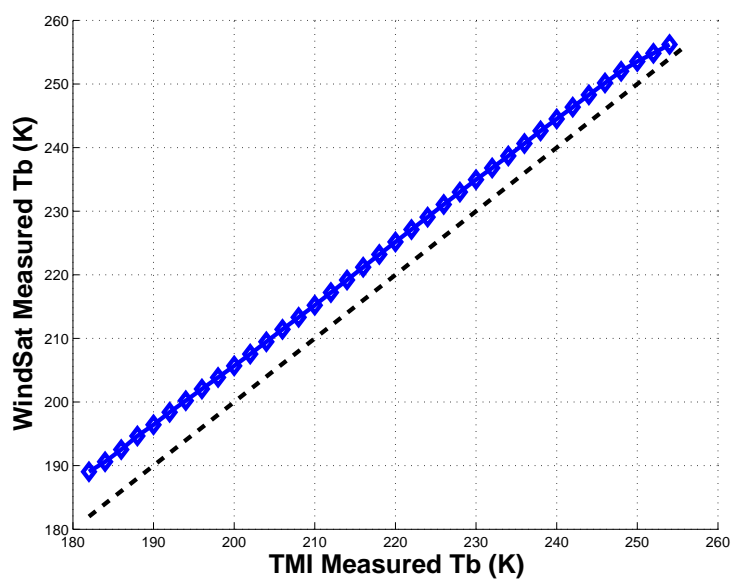

(a) Raw Tb observations.

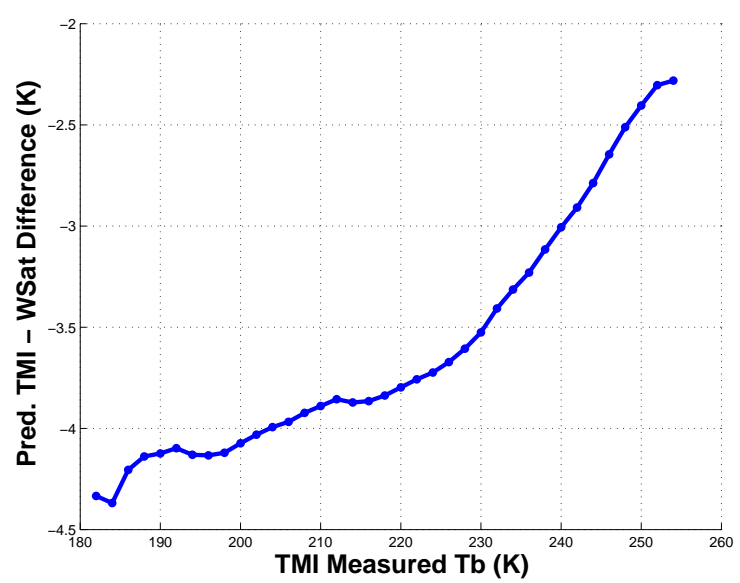

(b) Expected Tb difference predicted by RTM.

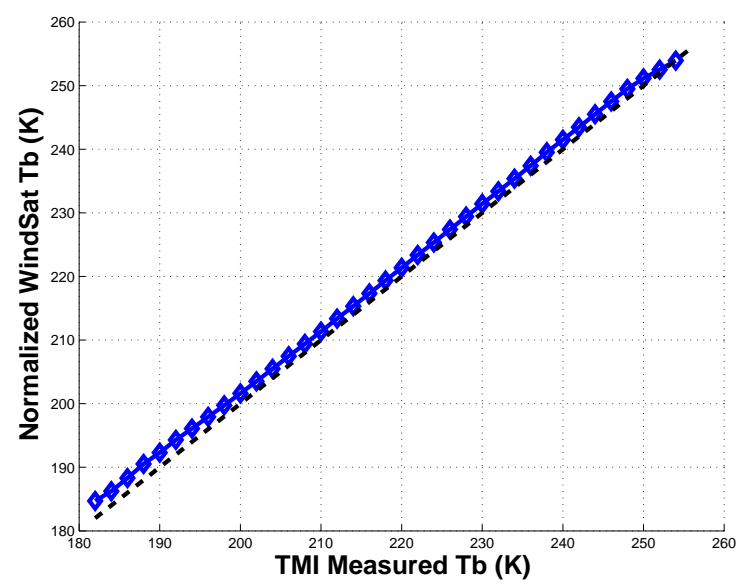

(c) Normalized WindSat and TMI Tb comparisons.

Figure B.4: Normalized WindSat 23V Tb observations to corresponding TMI channel frequency and incidence angle. 


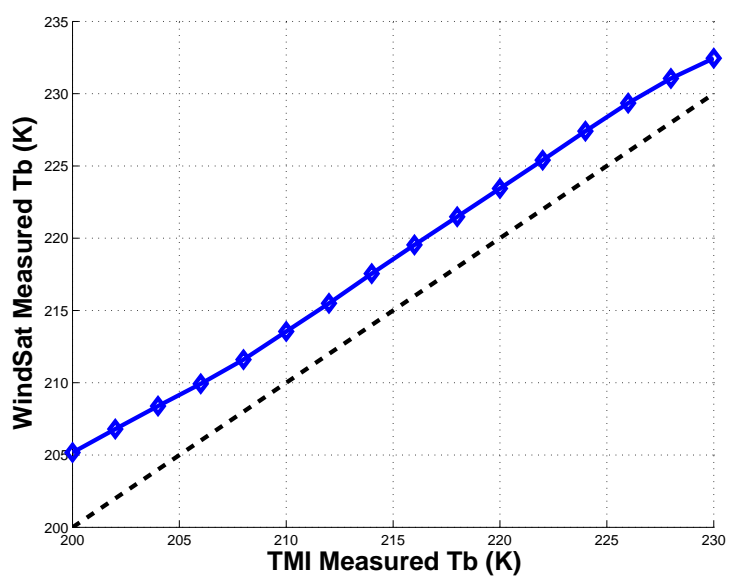

(a) Raw Tb observations.

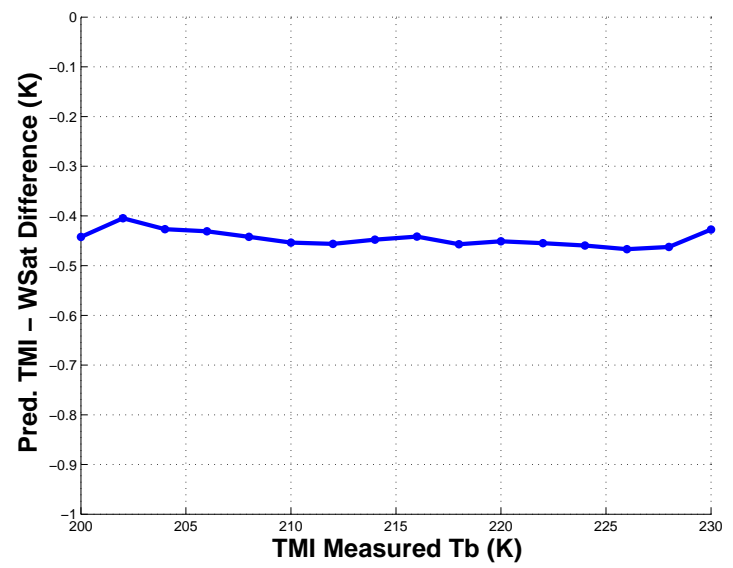

(b) Expected $\mathrm{Tb}$ difference predicted by RTM.

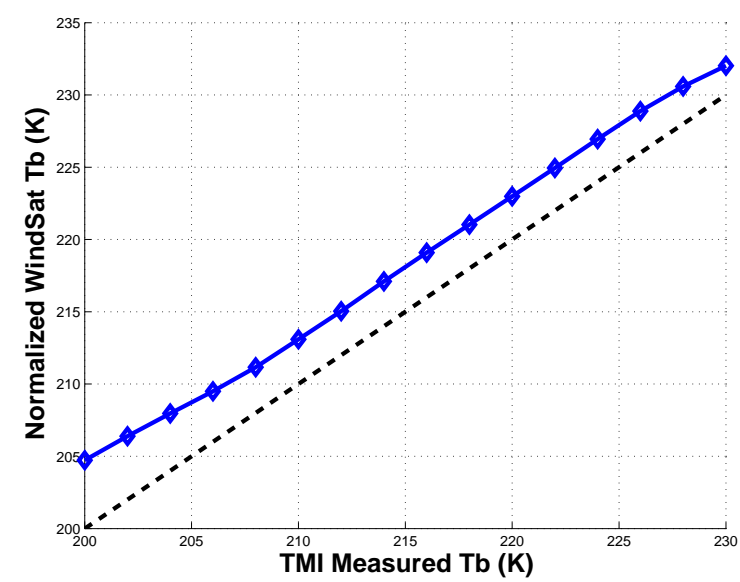

(c) Normalized WindSat and TMI Tb comparisons.

Figure B.5: Normalized WindSat 37V Tb observations to corresponding TMI channel frequency and incidence angle. 


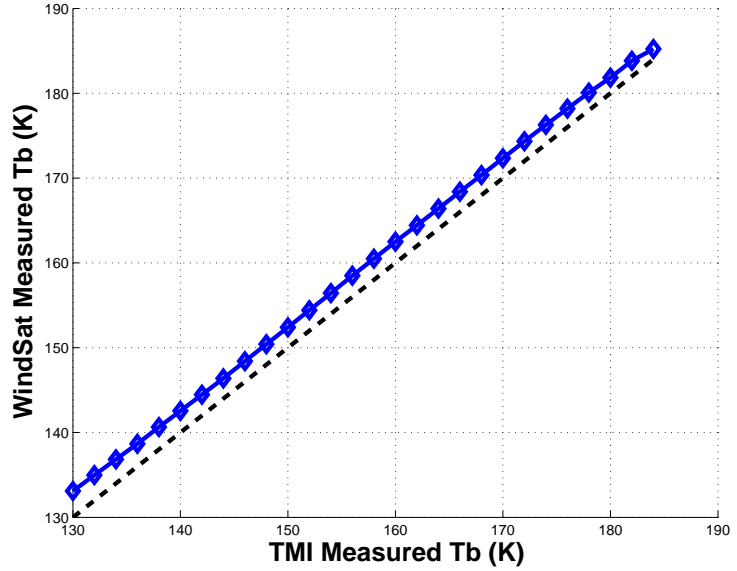

(a) Raw Tb observations.



(b) Expected Tb difference predicted by RTM.

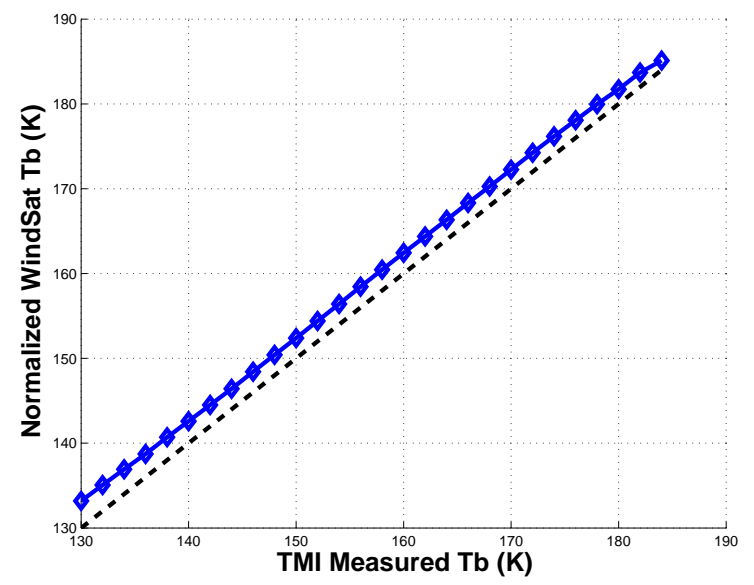

(c) Normalized WindSat and TMI Tb comparisons.

Figure B.6: Normalized WindSat 37H Tb observations to corresponding TMI channel frequency and incidence angle. 


\section{TMI OBSERVATIONS SEPARATED BY TIME OF DAY}

This appendix demonstrates the effect of splitting the TMI-WindSat collocations by separating the ascending and descending portions for WindSat, which correspond to local solar time of day (ascending passes of WindSat occur around 6:30 pm and the descending passes occur about 6:30 am). When the match-ups were sorted according to ascending and descending passes (local solar time), we calculated the Tb differences between TMI morning and evening match-ups. The average of all TMI Tb measurements for the ascending passes are $\sim 1.5 \mathrm{~K}$ higher than those of the descending passes for the $\mathrm{V}$-pol, and $\sim 1 \mathrm{~K}$ higher for the H-pol, as shown in the following figures.



Figure C.1: TMI 10V Tb observed Evening - Morning difference. 


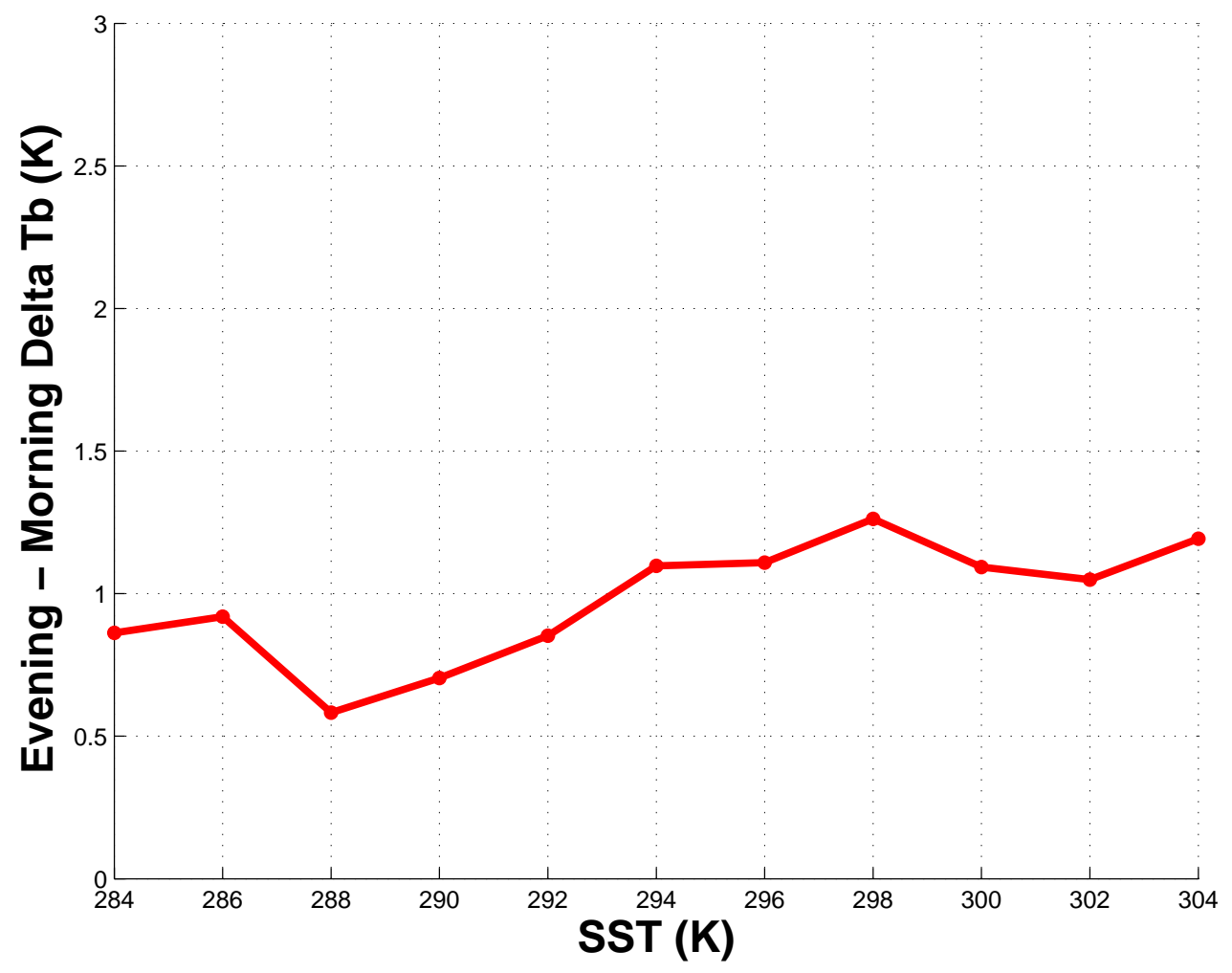

Figure C.2: TMI 10H Tb observed Evening - Morning difference. 




Figure C.3: TMI 19V Tb observed Evening - Morning difference. 


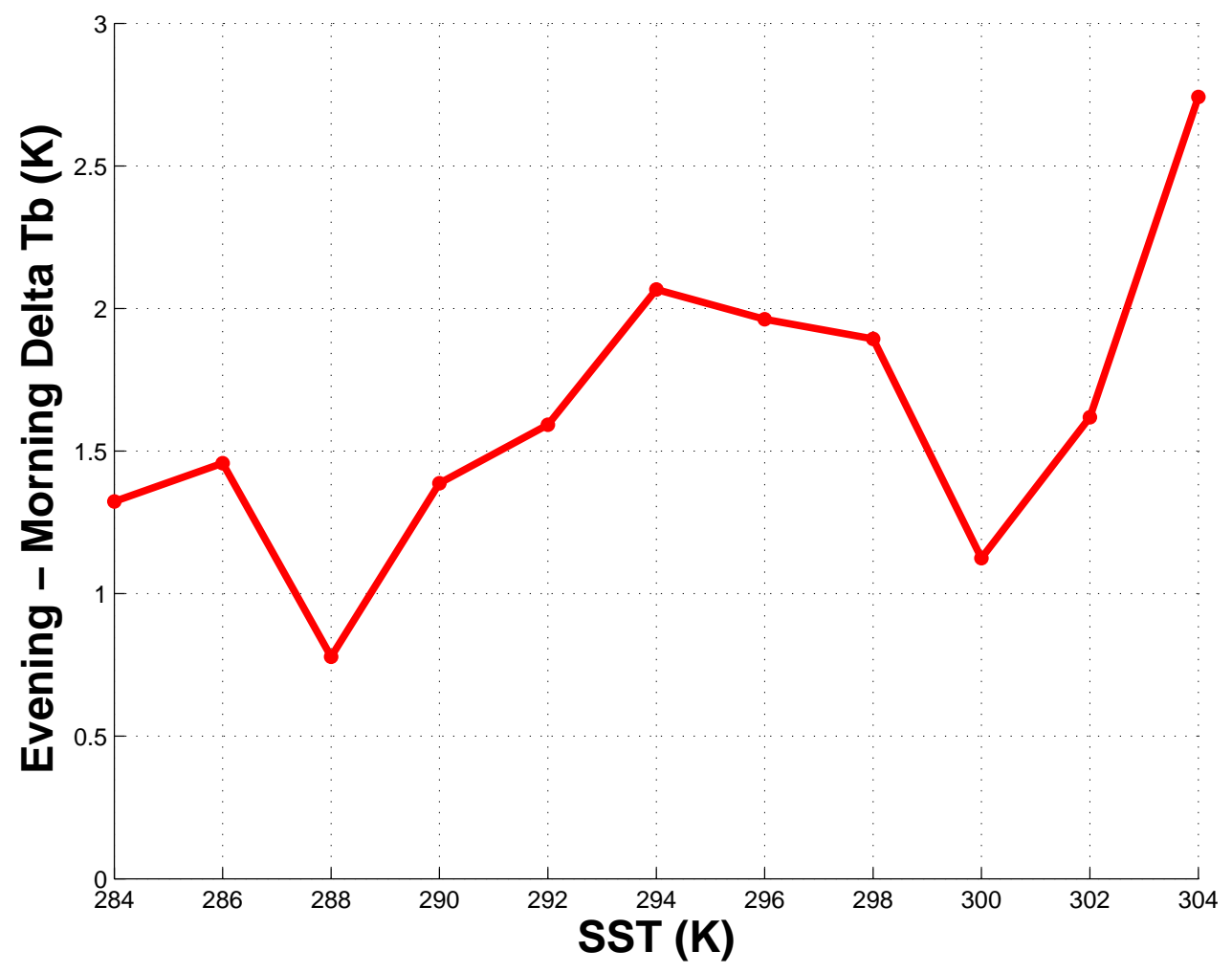

Figure C.4: TMI 19H Tb observed Evening - Morning difference. 


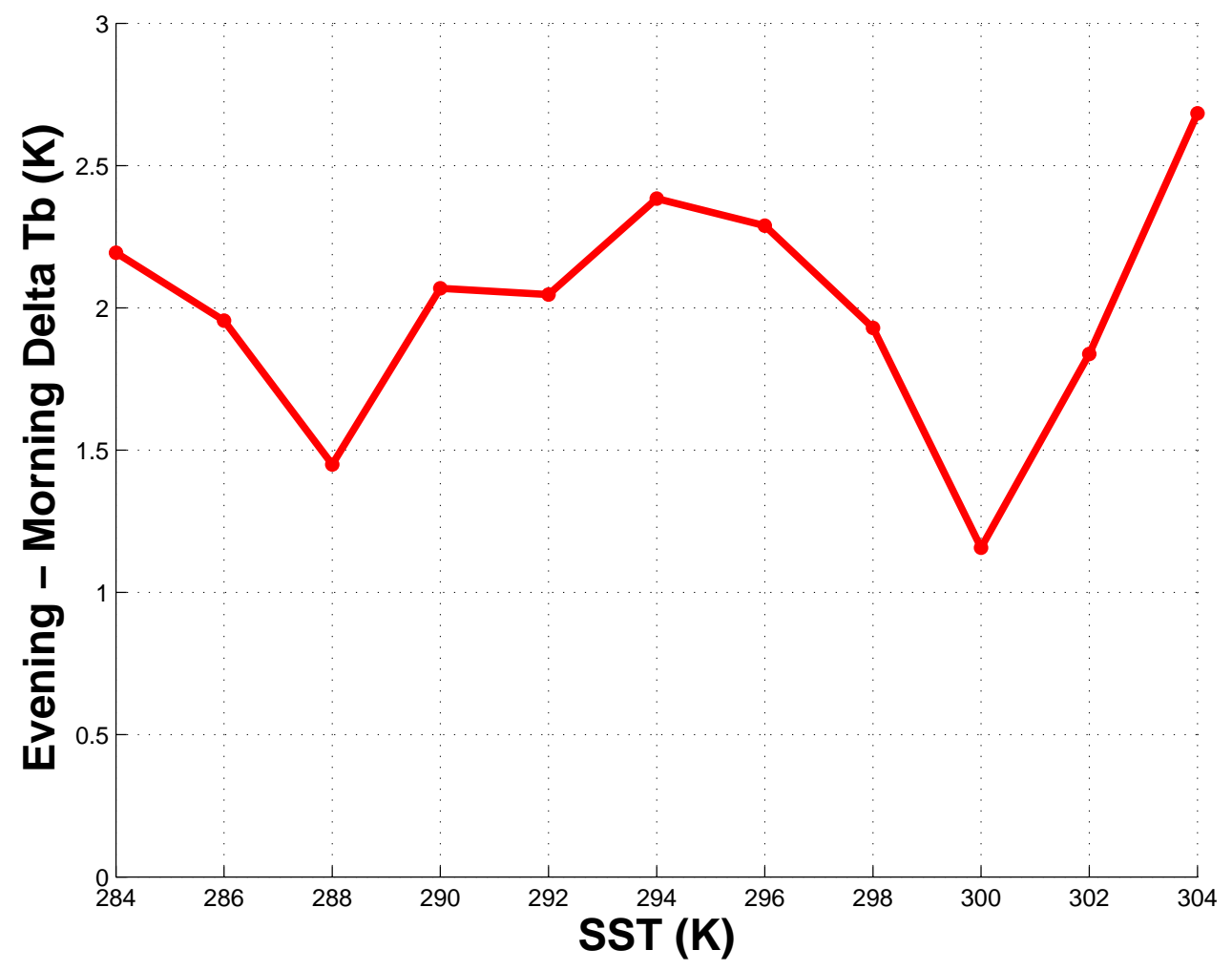

Figure C.5: TMI 21V Tb observed Evening - Morning difference. 


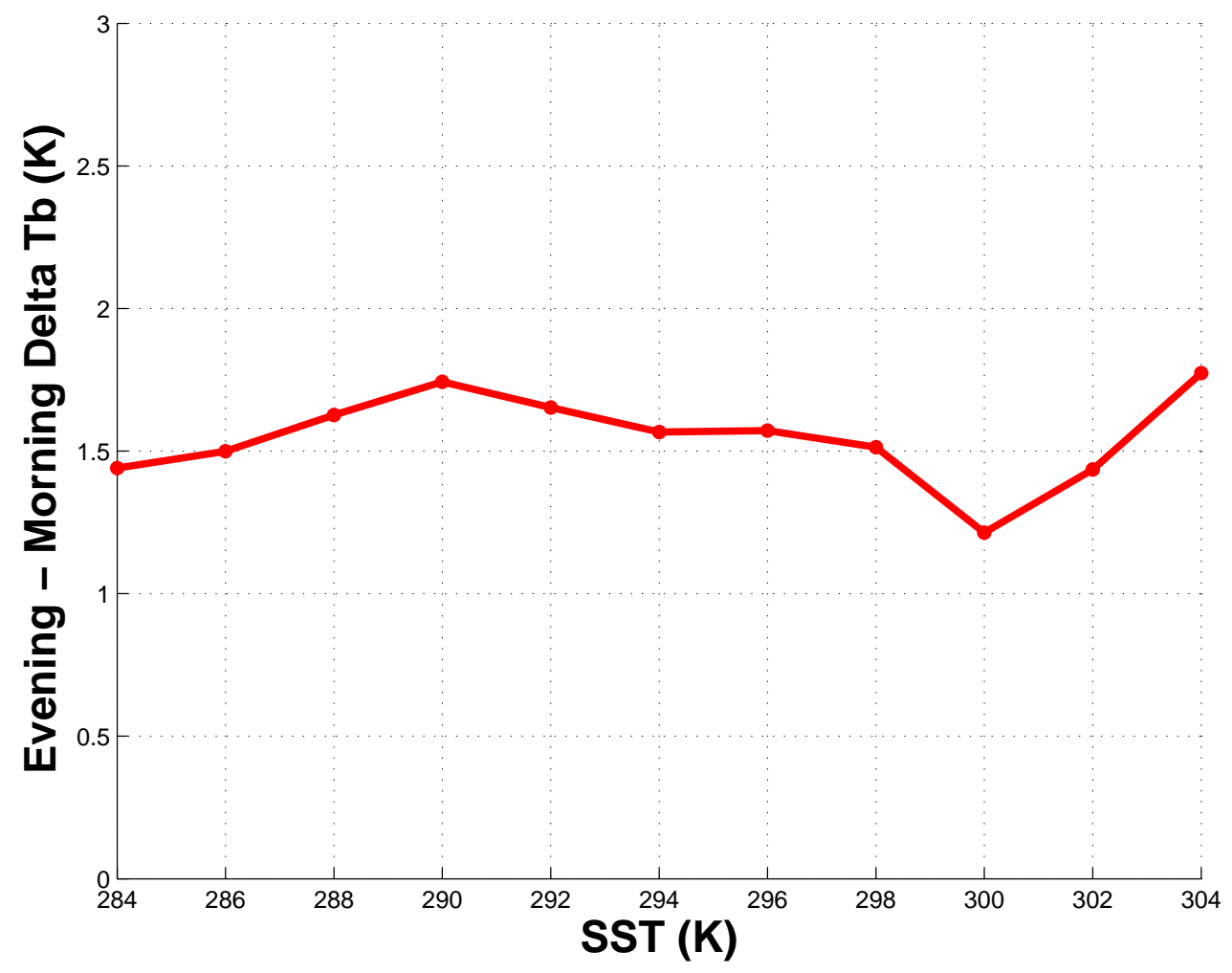

Figure C.6: TMI 37V Tb observed Evening - Morning difference. 


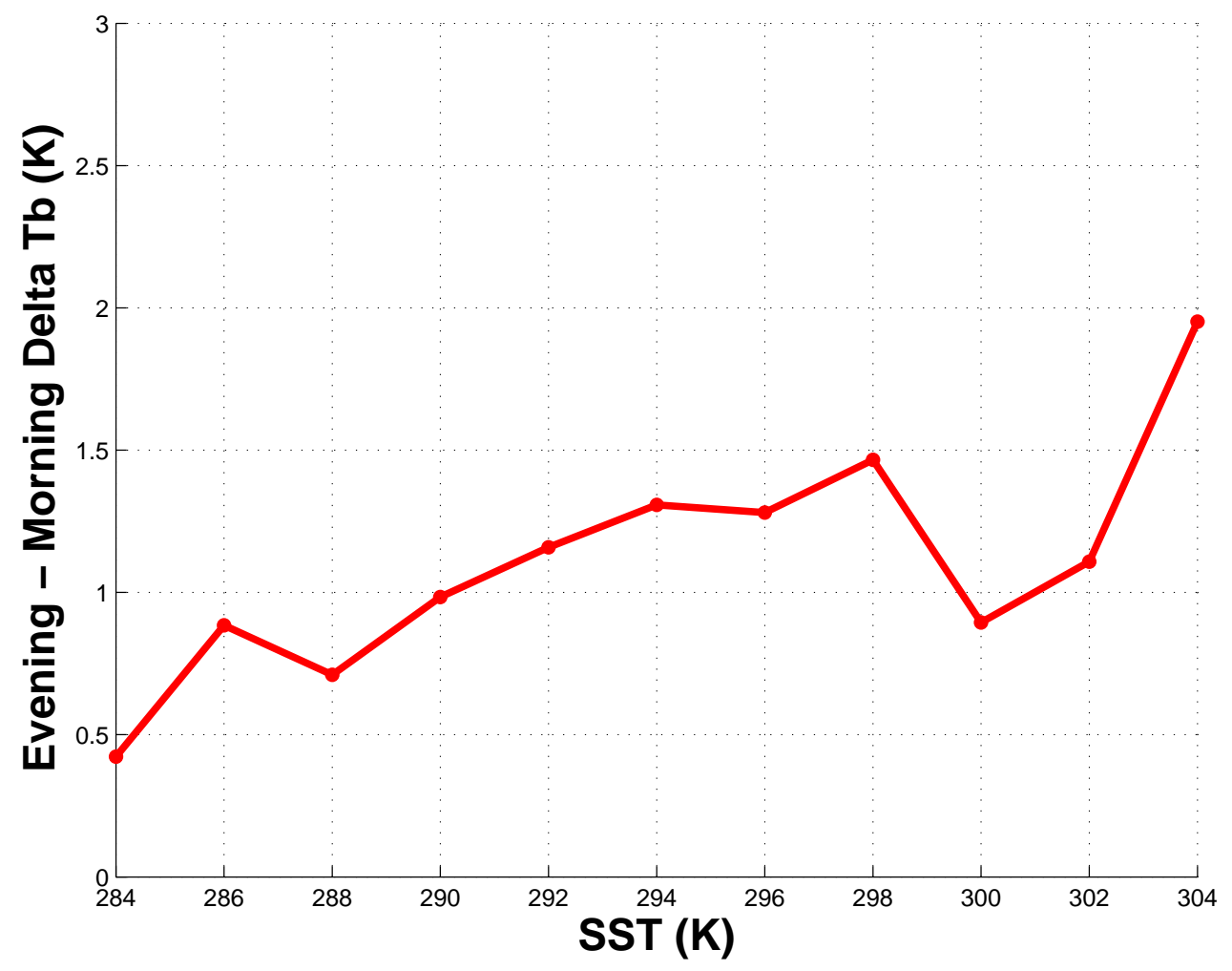

Figure C.7: TMI 37H Tb observed Evening - Morning difference. 


\section{EFFECT OF DERIVED TMI TB CORRECTION ON H-POL TMI-WINDSAT RADIOMETRIC BIASES}

The following figures show the H-pol radiometric biases between TMI and WindSat for both uncorrected and corrected TMI Tb's, separated by time of day. The uncorrected Tb's have time-of-day dependent differences of greater than $1 \mathrm{~K}$ in the biases, while the timeof-day dependence of the relative TMI/WindSat calibration reduces to less than $0.3 \mathrm{~K}$ for the corrected Tb's. 




(a) Uncorrected TMI Tb.



(b) Corrected TMI TB.

Figure D.1: Relative 10H radiometric biases with WindSat for uncorrected and corrected TMI Tb's. 


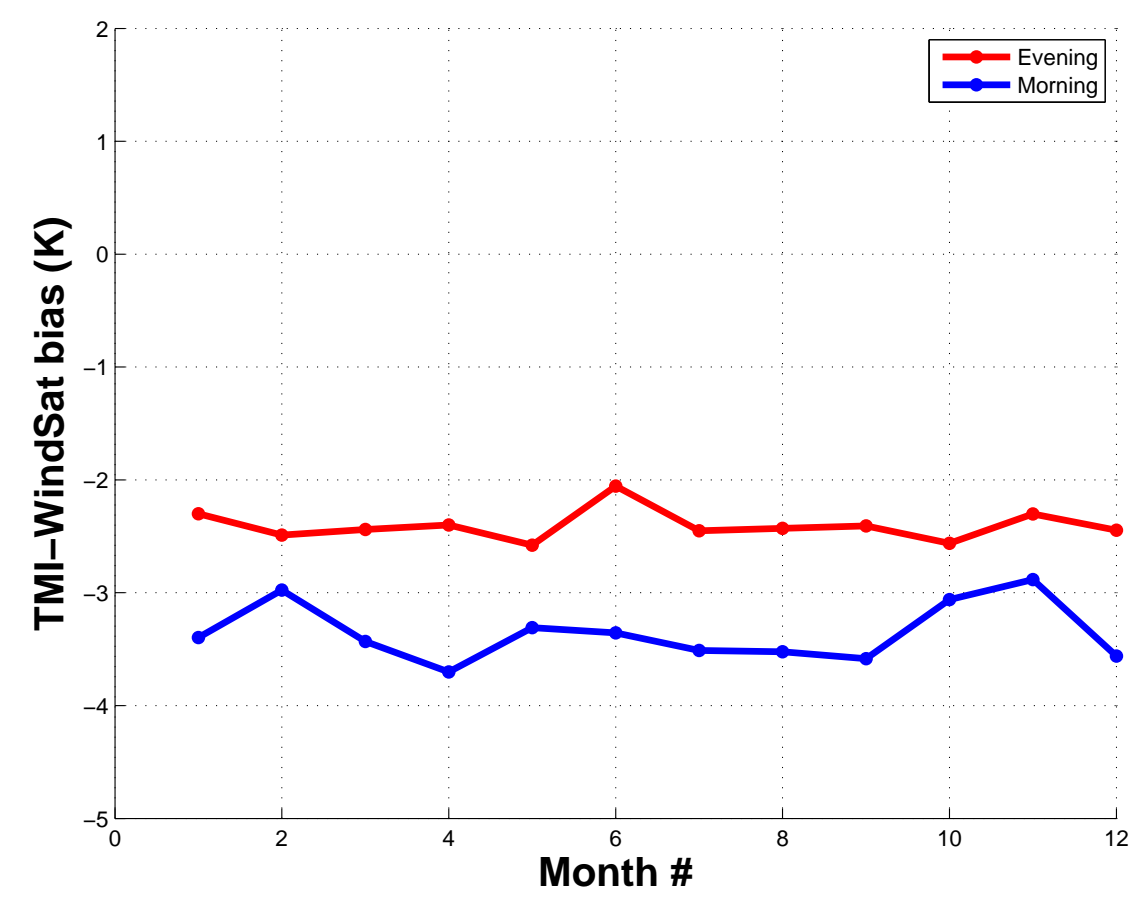

(a) Uncorrected TMI Tb.

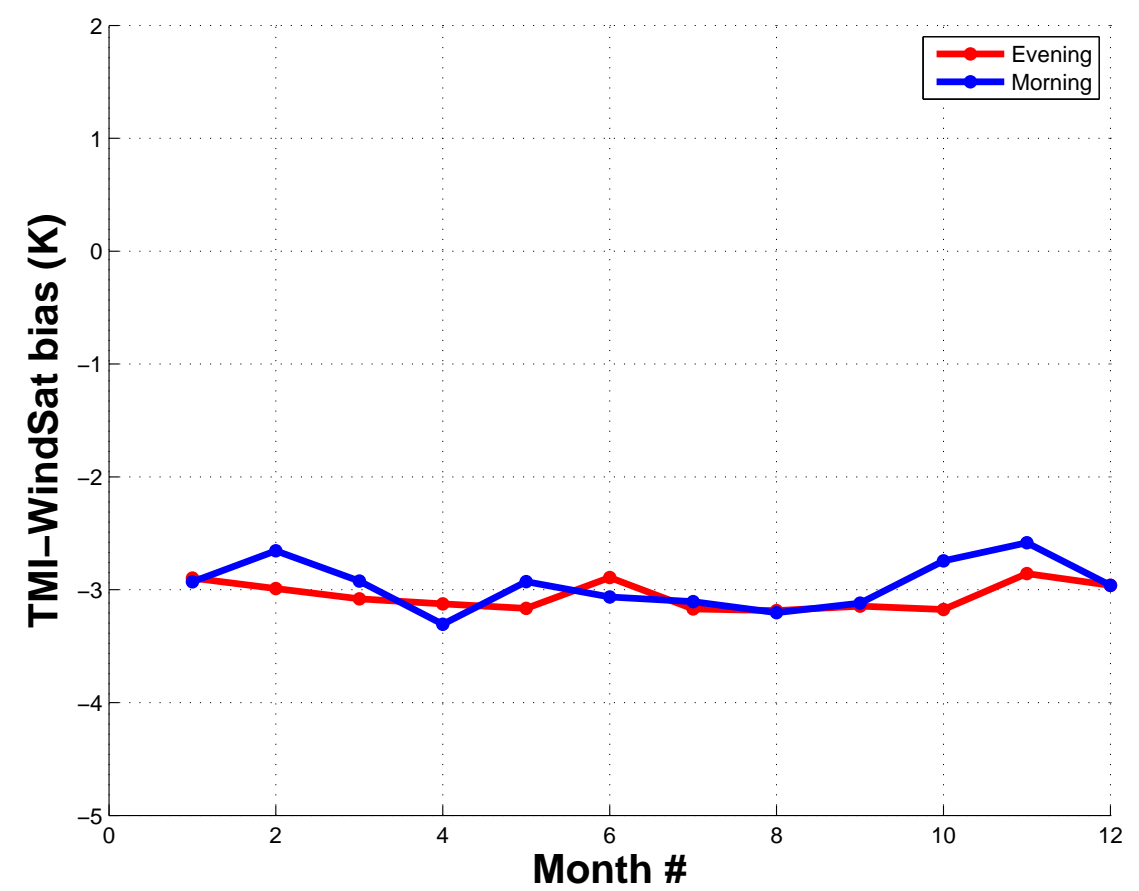

(b) Corrected TMI TB.

Figure D.2: Relative 19H radiometric biases with WindSat for uncorrected and corrected TMI Tb's. 




(a) Uncorrected TMI Tb.

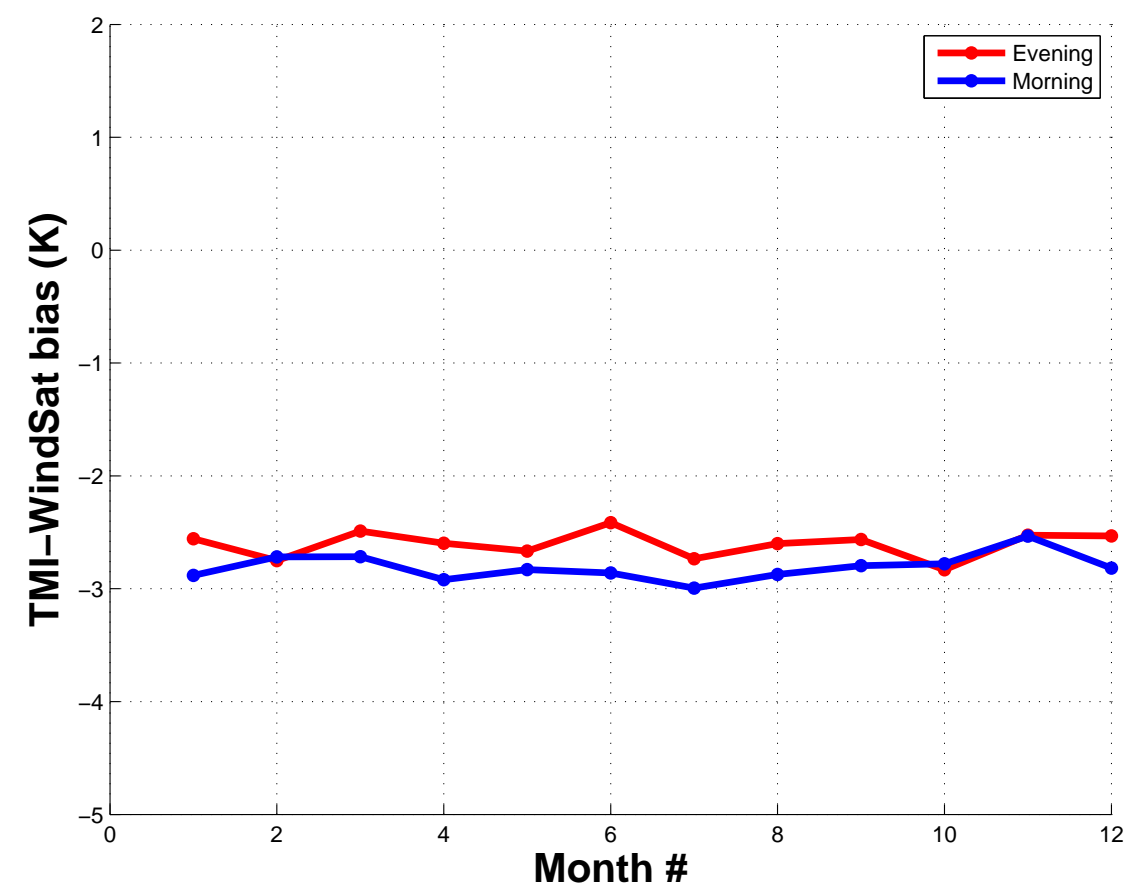

(b) Corrected TMI TB.

Figure D.3: Relative 37H radiometric biases with WindSat for uncorrected and corrected TMI Tb's. 


\section{LIST OF REFERENCES}

[1] http://trmm.gsfc.nasa.gov/, "Tropical Rainfall Measuring Mission."

[2] J. Simpson, C. Kummerow, W. K. Tao, and R. F. Adler, "On the Tropical Rainfall Measuring Mission (TRMM)," Met. and Atm. Phy., 1996.

[3] R. Adler, G. J. Huffman, D. Bolvin, S. Curtis, and E. J. Nelkin, "Tropical Rainfall Distributions Determined Using TRMM Combined with Other Satellite and Rain Gauge Information," J. of App. Met., 2000.

[4] National Research Council, "Assessment of the Benefits of Extending the Tropical Rainfall Measuring Mission: A Perspective from the Research and Operations Communities, Interim Report," http://books.nap.edu/catalog.php?record_id=11195, 2006.

[5] A. Lawler, "NASA satellite wins reprieve," Science, vol. 305, August 2004.

[6] http://www.nap.edu/catalog/11724.html, " NOAA's Role in Space-Based Global Precipitation Estimation and Application (Executive Summary)."

[7] F. Wentz, P. Ashcroft, and C. Gentemann, "Post-launch calibration of the TRMM microwave imager," Geoscience and Remote Sensing, IEEE Transactions on, vol. 39, no. 2, pp. 415-422, Feb 2001.

[8] L. Hong, W. L. Jones, and T. T. Wilheit, "Inter-Satellite Microwave Radiometer Calibration Between AMSR and TMI," IEEE International Geoscience and Remote Sensing Symposium, 2006.

[9] L. Hong, W. L. Jones, and T. Wilheit, "Inter-satellite radiometer calibrations between WindSat, TMI and AMSR," IEEE International Geoscience and Remote Sensing Symposium, 2007.

[10] L. Hong, "Inter-Satellite Microwave Radiometer Calibration," Ph.D. dissertation, University of Central Florida, 2008.

[11] L. Hong, W. L. Jones, T. T. Wilheit, and T. Kasparis, "Two Approaches for Intersatellite Radiometer Calibrations between TMI and WindSat," J. of Meteorological Society of Japan (JMSJ) special issue on Precipitation Measurements from Space, 2009, accepted for publication.

[12] K. Gopalan, L. Jones, T. Wilheit, and T. Kasparis, "Inter-Satellite Radiometer Calibration of Windsat, TMI AND SSMI," IEEE International Geoscience and Remote Sensing Symposium, 2008. 
[13] K. Gopalan, S. Biswas, W.L.Jones, and T. Kasparis, "Inter-Satellite Radiometric Calibration of WindSat, TMI and SSMI," PMM Internat. Sci. Team meeting, Ft. Collins CO, 2008 .

[14] K. Gopalan, L. Hong, W.L.Jones, and T. Kasparis, "Radiometric inter-calibration of TMI and WindSat: A GPM case study," TRMM Intl. Sci. Conf., Las Vegas, NV, 2008.

[15] T. Wilheit et al., "Use of TMI as a radiometric calibration transfer standard," TRMM Intl. Sci. Conf., Las Vegas, NV, 2008.

[16] T. T. Wilheit et al., "Cross-calibration of radiometers in GPM constellation," IEEE International Geoscience and Remote Sensing Symposium, 2008.

[17] http://mrain.atmos.colostate.edu/LEVEL1C/index.html, "Radiometer Level 1C data."

[18] Tropical Rainfall Measuring Mission Science Data and Information System (TSDIS), "Interface Control Specification Between the TSDIS and the TSDIS Science User (TSU)," vol. 3, 2007.

[19] ftp://ftp.orbit.nesdis.noaa.gov/pub/corp/scsb/wchen/doc/ssmitdr.html, "SSM/I Temperature Data Record (TDR) Data Sets."

[20] http://www.cpi.com/twiki/pub/WindSat/WindSatNews/SDR_Format_c200.pdf, "WindSat SDR Format."

[21] http://dss.ucar.edu/datasets/ds083.2/, "NCEP FNL Global Tropospheric Analyses, 1x1, daily 1999Jul30- present."

[22] http://wwwt.emc.ncep.noaa.gov/gmb/gdas/, "EMC : Global Data Assimilation Group."

[23] http://www.remss.com/, "Remote sensing systems."

[24] K. Hilburn and F. Wentz, "Intercalibrated passive microwave rain products from the unified microwave ocean retrieval algorithm (UMORA)," Journal of Applied Meteorology and Climatology, 2008.

[25] T. Wilheit, "Personal communication."

[26] F. Ulaby, R. K. Moore, and A. Fung, Microwave Remote Sensing - Active and Passive Volume I - Microwave Remote Sensing Fundamentals and Radiometry. Addison-Wesly, 1981.

[27] C. Elachi, Introduction to the Physics 83 Tech of Remote Sensing. John Wiley \& Sons, 1987.

[28] G. Elsaesser, "A parametric optimal estimation retrieval of the non-precipitating parameters over the global oceans," Master's thesis, Colorado State University, 2006. 
[29] P. W. Rosenkranz, "Water vapor microwave continuum absorption: A comparison of measurements and models," Radio Science, vol. 33, pp. 919-928, 1998.

[30] B. Geerts and E. Linacre, "The height of the tropopause," http://wwwdas.uwyo.edu/g̃eerts/cwx/notes/chap01/tropo.html, 1997.

[31] M. Wisler and J. P. Hollinger, "Estimation of marine environmental parameters using microwave radiometric remote sensing systems," NRL Memo Rpt, no. 3661, 1977.

[32] S. D. Thompson, "Evaluation of a microwave radiative transfer model for calculating satellite brightness temperature," Master's thesis, University of Central Florida, 2004.

[33] T. Wilheit, "Personal communication."

[34] A. Geer, "Solar dependent biases in microwave imager observations assimilated at ECMWF," ECMWF Research Department Memorandum, 2008.

[35] http://stk.com, "Satellite toolkit."

[36] S. Biswas, "Personal communication."

[37] K. Gopalan, L. Jones, S. Biswas, S. Bilanow, T. Wilheit, and T. Kasparis, "A TimeVarying Radiometric Bias Correction for the TRMM Microwave Imager," IEEE Transactions on Geoscience and Remote Sensing, 2008, accepted for publication.

[38] L. Jones, "Version-7 Brightness Temperature Correction Algorithm," CFRSL Technical Memorandum, 2008.

[39] http://meghatropiques.ipsl.polytechnique.fr/, "Megha-Tropiques : Water cycle and energy budget in the tropics."

[40] http://podaac.jpl.nasa.gov:2031/sensor_docs/ssmi.html, "Special Sensor Microwave Imager (SSM/I)."

[41] D. J. Cavalieri, "NASA Sea Ice Validation Program for the Defense Meteorological Satellite Program Special Sensor Microwave Imager: Final Report," NASA Technical memorandum, 1992.

[42] M. C. Colton and G. A. Poe, "Intersensor Calibration of DMSP SSM/Is: F-8 to F-14, 1987-1997," IEEE Transactions on Geoscience and Remote Sensing, vol. 37, 1999.

[43] C. Kummerow et al., "The Tropical Rainfall Measuring Mission (TRMM) Sensor Package," Journal of Atmospheric and Oceanic Technology, 1992.

[44] http://www.nrl.navy.mil/WindSat/Description.php, "WindSat Payload description."

[45] P. Gaiser et al., "The WindSat Spaceborne Polarimetric Microwave Radiometer: Sensor Description and Early Orbit Performance," IEEE Transactions on Geoscience and Remote Sensing, vol. 42, 2004. 